A COMPUTATIONAL ANALYSIS OF NANOPARTICLE-MEDIATED

OPTICAL BREAKDOWN

by

Yevgeniy R. Davletshin

Master of Science, Ryerson University, 2011

Bachelor of Science, Al-Farabi Kazakh National University, 2003

A dissertation

presented to Ryerson University

in partial fulfillment of the

requirements for the degree of

Doctor of Philosophy

in the Program of

Biomedical Physics

Toronto, Ontario, Canada, 2017

(C)Yevgeniy R. Davletshin 2017 



\section{AUTHOR'S DECLARATION}

I hereby declare that I am the sole author of this dissertation. This is a true copy of the thesis, including any required final revisions, as accepted by my examiners.

I authorize Ryerson University to lend this dissertation to other institutions or individuals for the purpose of scholarly research.

I further authorize Ryerson University to reproduce this dissertation by photocopying or by other means, in total or in part, at the request of other institutions or individuals for the purpose of scholarly research.

I understand that my dissertation may be made electronically available to the public. 



\title{
A COMPUTATIONAL ANALYSIS OF NANOPARTICLE-MEDIATED OPTICAL
}

\section{BREAKDOWN}

\author{
Doctor of Philosophy 2017 \\ Yevgeniy R. Davletshin \\ Biomedical Physics \\ Ryerson University
}

\begin{abstract}
A theoretical model of the optical breakdown phenomena during picosecond and femtosecond laser pulse exposure with gold nanoparticles in water was developed. The model provides new and valuable insight into the dependence of the optical breakdown on the wavelength, morphology and environment in the vicinity of the nanoparticles. The developed model was successfully validated against experimental data, which also revealed some insights to the criterion for optical breakdown. Three studies were performed using the model.

In the first study, the effects of the dielectric environment on the optical extinction spectra of individual bare and silica-coated gold nanorods were examined. The experimental extinction spectra of an individual gold nanorod was compared to a calculation from a numerical model that included environmental features present in the measurements and the morphology of the corresponding nanorod measured by transmission elec-
\end{abstract}


tron microscopy. The combination of these experimental and theoretical tools permitted a detailed interpretation of the optical properties of an individual gold nanorod.

In the second study, a strongly coupled finite element model of nanoparticle-mediated optical breakdown phenomena was developed. This model was used to theoretically study a 6 ps laser pulse interaction with uncoupled and plasmon coupled gold nanoparticles. The study showed how the one-dimensional assembly of nanoparticles affects the optical breakdown threshold of its surroundings. The optical breakdown threshold had a stronger dependence on the optical near-field enhancement than on the volume of the nanostructure or its absorption cross-section.

Finally, a model was developed to study the wavelength dependence of the threshold of gold nanorod-mediated optical breakdown during picosecond and femtosecond near infrared optical pulses. This study showed that the wavelength dependence in the picosecond regime is governed solely by the changes of the nanorod's optical properties. On the other hand, the optical breakdown during femtosecond pulse exposures was found to depend on the multiphoton ionization and its wavelength dependence when, $E_{\text {ratio }}$, the ratio of the maximum electric field from the outside to the inside of the nanorod was greater than 7 .

The developed model and conducted research deepens the understanding of the nanoparticlemediated optical breakdown in water and updates the theoretical formulation of the process with the latest findings, which leads to advancing this technology further. 


\section{Acknowledgements}

Firstly, I would like to express my sincere gratitude to my advisor Dr. J. Carl Kumaradas for the continuous support of my Ph.D. study and related research, for his patience, motivation, and immense knowledge. His guidance helped me in the research and writing of this thesis. I could not imagine having a better advisor and mentor for my Ph.D. study.

Besides my advisor, I would like to thank the rest of my thesis committee: Dr. Michael Kolios and Dr. Renat R. Letfullin for their insightful comments and encouragement, but also for the hard questions which incented me to widen my research from various perspectives.

I also would like to thank Dr. Bo Tan on accepting the role of external examiner, reading and commenting my dissertation.

My sincere thanks also goes to Dr. Natalia Del Fatti and Dr. Fabrice Vallee, who provided me an opportunity to join their team as intern, and who gave access to the laboratory and research facilities. Without their precious support it would not have been possible to conduct this research.

I would also like to thank Dr. Norbert Linz and Dr. Alfred Vogel for their fruitful discussions.

The final thanks goes to my friends that I met during graduate school. 



\section{Dedication}

This dissertation is dedicated to my mother and father, whose love, passion, kindness, support and wisdom I carry on forever in my heart and soul. I would like to dedicate this research also to my sweet and lovely sister who is always there for me when I need her. And finally I would like to dedicate this degree to you my love - Firuza. Without your love and support neither this degree or research would be ever possible. Thank you for accepting my heart and soul. Our marriage and the birth of our son were the most important events during my graduate studies. 



\section{Contents}

Declaration .............................. ii

Abstract . . . . . . . . . . . . . . . . . . . . v

Acknowledgements ................................ vii

Dedication . . . . . . . . . . .......... ix

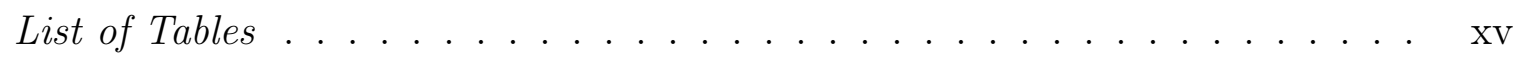

List of Figures . . . . . . . . . . . . . . . . . . . xvii

$\begin{array}{lll}1 & \text { Introduction } & 1\end{array}$

1.1 Nanoparticle-mediated optical breakdown and its applications . . . . . . 1

1.2 The mechanism of nanoparticle-mediated optical breakdown . . . . . . . 3

1.2 .1 Optical behaviour of nanoparticles . . . . . . . . . . . 4

$\begin{array}{lll}1.2 .2 & \text { The process of nanoparticle-mediated optical breakdown in water } & 5\end{array}$

$\begin{array}{lll}1.2 .2 .1 & \text { The wavelength dependence of band structure of water . } & 6\end{array}$

1.3 Motivation . . . . . . . . . . . . . . . . . . . . . . 7

1.3 .1 Modelling laser induced optical breakdown (LIOB) $\ldots \ldots \ldots .7$

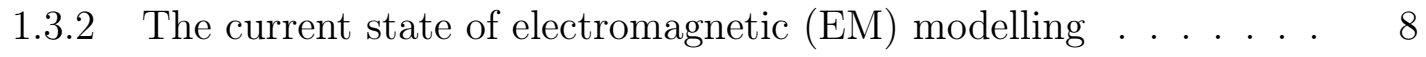

1.3 .3 The current state of LIOB modelling . . . . . . . . . . . . . . 9

1.3 .4 Challenges in a computational analysis . . . . . . . . . . 13

1.3.4.1 Optical characterization of a nanoparticle . . . . . . 13

1.3 .4 .2 Laser source. . . . . . . . . . . . . . . . . . 15

1.3.4.3 Strongly coupled multiphysics phenomena . . . . . . . 15

1.4 Thesis objective and specific aims $\ldots \ldots \ldots \ldots$

1.5 Thesis organization $\ldots \ldots \ldots \ldots \ldots \ldots$ 
\begin{tabular}{lll}
\hline 2 & Theory & 19
\end{tabular}

2.1 The electromagnetic model . . . . . . . . . . . . . . . . . . . . . . . . . . . 19

2.1 .1 Size corrections to bulk dielectric function . . . . . . . . . . 20

2.2 The two-temperature model (TTM) of a gold nanoparticle heating . . . . 22

2.3 Heat transfer (HT) in water . . . . . . . . . . . . . . . . . . 24

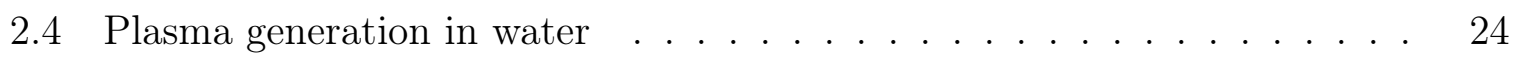

2.4 .1 The full plasma rate equation $\ldots \ldots \ldots \ldots \ldots$

2.4 .2 Photoionization . . . . . . . . . . . . . . . . 27

2.4 .3 Avalanche ionization . . . . . . . . . . . . . . 28

2.4 .4 Thermal ionization and photo-thermal emission . . . . . . . . . 29

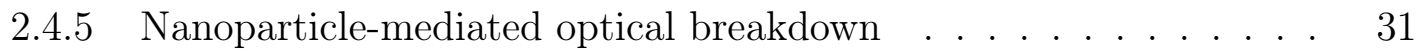

2.4 .6 The wavelength dependence of optical breakdown in water . . . . 32

$\begin{array}{lll}3 & \text { Methods } & 37\end{array}$

3.1 A finite element method based model . . . . . . . . . . . . . . . . . 37

3.2 The role of the environment on the optical properties of gold nanorods . 39

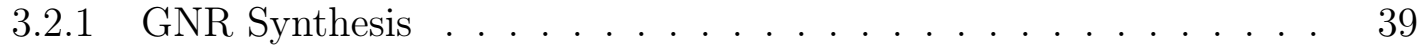

3.2 .2 Optical Characterization $\ldots \ldots \ldots \ldots \ldots$

3.2 .3 Finite element model . . . . . . . . . . . . . . . . . . . . 43

3.3 The role of gold nanoparticle morphology and coupling in optical breakdown during picosecond pulse exposures $\ldots \ldots \ldots$. . . . . . . 49

3.3 .1 The electromagnetic model for LIOB $\ldots \ldots \ldots$

3.3 .2 A two-temperature model (TTM) of nanoparticle heating . . . . . 51

3.3 .3 HT in the medium . . . . . . . . . . . . . . 51

3.3 .4 Plasma dynamics $\ldots \ldots \ldots \ldots \ldots$. . . . . . . . . . . . . . 51

3.4 The wavelength dependence of gold nanorod-mediated optical breakdown

with infrared ultrashort pulses . . . . . . . . . . . . . . . . 53

3.5 Validation of the full model $\ldots \ldots \ldots \ldots \ldots$

$\begin{array}{llr}4 & \text { Results } & 59\end{array}$

4.1 Validation of the model . . . . . . . . . . . . . . . . . . . . . 59

4.1 .1 Validation of the EM model . . . . . . . . . . . . . . . . 59

4.1 .2 Validation of the combined EM and TTM model . . . . . . . . . 61 
4.1 .3 Validation of the full model . . . . . . . . . . . . . . 63

4.2 The role of the environmental on the optical properties of gold nanorods 66

4.3 The role of gold nanoparticle morphology and coupling in optical break-

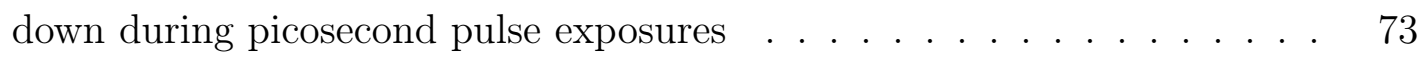

4.4 The wavelength dependence of gold nanorod-mediated optical breakdown with infrared ultrashort pulses . . . . . . . . . . . . . . 83

$\begin{array}{lll}5 & \text { Summary, Discussion and Conclusions } & 93\end{array}$

5.1 Validation of LIOB model . . . . . . . . . . . . . . . . . . . . . . . . . 93

$5.2 \quad$ Role of environment on optical properties of a single nanoparticle $\ldots \ldots \ldots \ldots \ldots$. . . . . . . . . . 96

5.3 Morphology dependence of LIOB $\ldots \ldots \ldots \ldots$

5.4 Wavelength dependence of LIOB $\ldots \ldots \ldots \ldots$

$5.5 \quad$ Key contributions $\ldots \ldots \ldots \ldots . \ldots \ldots$

$\begin{array}{lll}6 & \text { Future work } & 101\end{array}$

$6.1 \quad$ A new criterion for optical breakdown . . . . . . . . . . . . . . . . . . . 101

6.2 Sequence of pulses LIOB modelling . . . . . . . . . . . . . . . . . . . . 104

6.3 Combination of morphology and wavelength dependence study of LIOB . . . . . . . . . . . . . . . . . . . . . . 104

$6.4 \quad$ Nanoparticle-mediated optical breakdown during nanosecond pulse exposure 104

6.5 Thermal explosion and melting of nanoparticle . . . . . . . . . . . 105

$6.6 \quad$ Temperature dependent optical properties of gold $\ldots \ldots \ldots \ldots$

6.7 Thermionic emission and charging of the nanoparticle . . . . . . . . 106

\begin{tabular}{ll}
\hline References & 132
\end{tabular}

\begin{tabular}{|ll}
\hline Acronyms & 135
\end{tabular} 



\section{List of Tables}

1.1 Currently published models of a nanoparticle-mediated optical breakdown 12

3.1 Dimesions of the silica-coated and bare GNRS used in the model . . . . . 43

3.2 Parameters that were used in the electromagnetic model. . . . . . . . . . 48

3.3 Parameters that were used in two-temperature model (TTM) and heat transfer (HT) model. . . . . . . . . . . . . . . . . . . 52

3.4 Parameters that were used in plasma model. . . . . . . . . . . . . . . . 54

3.5 Parameters that were used in the plasma model for silica-core domain. . $\quad 57$

4.1 Gold nanoparticle types and dimensions . . . . . . . . . . . . . . 78

4.2 Effect of a dielectric shell on optical properties of a gold nanosphere trimer and nanorod . . . . . . . . . . . . . . . . . . . . . . . . 83

5.1 Currently published models of a nanoparticle-mediated optical breakdown 99 



\section{List of Figures}

2.1 Relative electric field enhancement, $\hat{\mathrm{E}}$, of a gold nanorod and nanosphere 20

$2.2 \quad$ A schematic of the laser induced optical breakdown processes in water. . 25

2.3 Schematic of two regimes of nanoparticle-mediated optical breakdown. . . 31

2.4 Ionization and geminate recombination pathways in liquid water . . . . . 33

2.5 Wavelength dependence of avalanche ionization (AI) and multiphoton ionization (MPI) rates . . . . . . . . . . . . . . . . . . . 34

$2.6 \quad$ Wavelength dependence of the threshold, $I_{\mathrm{th}}(\lambda)$, for plasma-mediated bub-

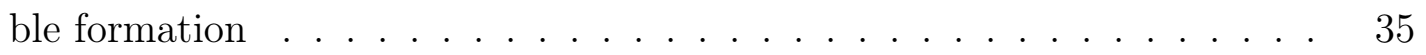

$2.7 \quad$ Comparison of predictions for $I_{\mathrm{th}}(\lambda)$ based on simplified optical breakdown model, with the experimentally determined spectrum $\ldots \ldots \ldots . .36$

$3.1 \quad$ A schematic of the laser induced optical breakdown (LIOB) model coupling. 38

3.2 transmission electron microscopy (TEM) images of the six investigated single uncoated and silica-coated gold nanorods and examples of optical and magnified view spatial modulation spectroscopy (SMS) images. . . . 41

3.3 The spatial modulation spectroscopy setup . . . . . . . . . . . . . . . 42

3.4 Schematics of the computational geometry . . . . . . . . . . . . . 47

$3.5 \quad$ Schematic of the optical breakdown finite element (FE) model geometry for a nanosphere trimer . . . . . . . . . . . . . 50

3.6 Schematic of the model geometry for gold nanorod $\ldots \ldots \ldots \ldots$

4.1 Validation of computational model against Mie solution . . . . . . . . . . 60

4.2 Calculated extinction cross-sections and near-field enhancements of gold nanoshells . . . . . . . . . . . . . . . . . . . . 61

4.3 Validation of EM and TTM model combination against experimental data 62 
4.4 A comparison of simulation against experimental data, case NS800 . . . . 64

4.5 A comparison of simulation against experimental data, case NS660 . . . . 65

4.6 The relative electric field enhancement of bare and silica coated gold nanorods on a substrate . . . . . . . . . . . . . . . 67

4.7 Comparison of simulated and experimental spectra of uncoated and silica coated gold nanorods . . . . . . . . . . . . . . . . . . 68

4.8 Simulated environmental effect on extinction cross-section of bare and silica coated gold nanorods . . . . . . . . . . . . . . . . . . . 70

$4.9 \quad$ A study of how the surroundings of a silica-coated gold nanorod (GNR) and a bare GNR affect $\lambda_{\mathrm{R}}$ and full-width at half-maximum (fwhm) . . . 71

4.10 Relative electric field enhancement and the log-scale of free electron density in the vicinity of a nanostructure . . . . . . . . . . . . . . . . . . . . . 74

4.11 Maximum near-field enhancement for different nanostructures . . . . . . 76

4.12 Nanoparticles of different morphology used in the model and corresponding parameters obtained to reach optical breakdown . . . . . . . . . . . . . . 77

4.13 Calculated free electron densities for different nanoparticle morphology and wavelengths exposed to 6 ps laser pulse at different fluencies . . . . . 79

4.14 The optical breakdown threshold is plotted against corresponding (a) nanoparticle volume, absorption cross sections, and maximum near-field enhancement . . . . . . . . . . . . . . . . . . . . 80

4.15 Maximum near-field enhancement and absorption cross-section of a $25 \mathrm{~nm}$ gold nanosphere trimer . . . . . . . . . . . . . . . . . . . 82

4.16 Absorption cross-section, $\sigma_{\text {abs }}$ (a) and a maximum electric field enhancement, $E_{\mathrm{e}, \max }(\mathrm{b})$ of a longitudinally polarized light of a $35 \mathrm{~nm}\left(\mathrm{GNR}_{35}\right)$ (green) and a $55 \mathrm{~nm}\left(\mathrm{GNR}_{55}\right)$ long gold nanorod (red). . . . . . . . . . . 84

4.17 LIOB intensity threshold for a 60 fs and 6 ps laser pulses versus absorption cross-section, of the 35 and $55 \mathrm{~nm}$ GNR and a maximum electric field enhancement ....................... . . 85

4.18 LIOB threshold $I_{\mathrm{th}}^{6 \mathrm{ps}}$ for a 6 ps and $I_{\mathrm{th}}^{60 \text { fs }}$ for a 60 fs pulse interaction with a GNR versus the wavelength of incidence . . . . . . . . . . . 87

4.19 Electric near field enhancement plotted for $\mathrm{GNR}_{35}$ and $\mathrm{GNR}_{55}$ at 750 and $975 \mathrm{~nm}$ wavelengths, before formation of the free electron plasma . . . . 88 
4.20 Plots of free electron density, generated in the vicinity of $\mathrm{GNR}_{35}$ exposed at $940 \mathrm{~nm} \ldots \ldots \ldots$. . . . . . . . . . . . . . . . 89

4.21 Free electron plasma density generation related to contribution to the free electron plasma generation through the MPI and photo-thermal emission

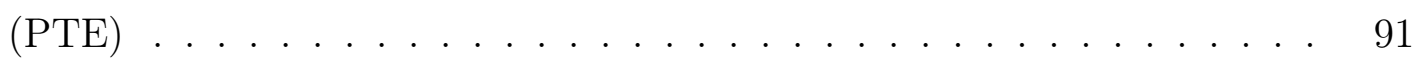

5.1 A comparison of the maximum free electron density calculated in the vicinity of gold nanoshells with the perforation fraction . . . . . . . . . . . . . 95

6.1 Calculated maximum temperature, $T_{\max }$, in the vicinity of the gold nanoshell (NS660 and NS800) at the end of the 70 fs pulse . . . . . . . . . . . . . . 103

6.2 Dependence of near-field enhancement, $E / E_{\text {inc }}$, in the vicinity of gold nanorod with uniform temperature of GNR $\left(T_{\mathrm{au}}\right)$ at plasmon resonance wavelength, $\lambda=860 \mathrm{~nm}$. . . . . . . . . . . . . . . . . . 107 



\section{Nomenclature}

\section{Notation}

$\hat{a} \quad$ Basis/unit vector

a $\quad$ Complex quantity

A Scalar quantity

A Vector quantity

Operators

$\Im(a)$ Imaginary part of $a$

$\nabla \cdot \mathbf{A}$ Divergence of $\mathbf{A}$

$\nabla \times \mathbf{A}$ Curl of $\mathbf{A}$

$\nabla A \quad$ Gradient of $A$

$\Re(a)$ Real part of $a$

| A | Magnitude of $\mathbf{A}$

$\tilde{A}^{*} \quad$ Complex conjugate of $\tilde{A}$

\section{Physical Quantities}
B Magnetic induction (T)
D Electric induction $\left(\mathrm{C} \cdot \mathrm{m}^{-2}\right)$ 
E Total electric field $\left(\mathrm{V} \cdot \mathrm{m}^{-1}\right)$

$\mathbf{E}_{\text {inc }}$ Incident electric field $\left(\mathrm{V} \cdot \mathrm{m}^{-1}\right)$

$\mathbf{E}_{\text {sca }} \quad$ Scattered electric field $\left(\mathrm{V} \cdot \mathrm{m}^{-1}\right)$

H Total magnetic field $\left(\mathrm{A} \cdot \mathrm{m}^{-1}\right)$

$\mathbf{H}_{\text {sca }} \quad$ Scattered magnetic field $\left(\mathrm{A} \cdot \mathrm{m}^{-1}\right)$

J Current density $\left(\mathrm{A} \cdot \mathrm{m}^{-2}\right)$

P Polarization $\left(\mathrm{C} \cdot \mathrm{m}^{-2}\right)$

S Poynting vector $\left(\mathrm{W} \cdot \mathrm{m}^{-2}\right)$

$\mathrm{S}_{\mathrm{av}} \quad$ Time-averaged Poynting vector $\left(\mathrm{W} \cdot \mathrm{m}^{-2}\right)$

$\chi \quad$ Electric susceptibility (1)

$\chi^{\mathrm{D}} \quad$ Drude (free electrons) susceptibility (1)

$\chi^{\mathrm{IB}} \quad$ Interband susceptibility (1)

$\chi_{m} \quad$ Magnetic susceptibility (1)

$\Gamma \quad$ Phenomenological damping constant $\left(\mathrm{s}^{-1}\right)$

$\gamma_{0} \quad$ Bulk damping constant $\left(\mathrm{s}^{-1}\right)$

$\kappa \quad$ Wavenumber $\left(\mathrm{m}^{-1}\right)$

$\kappa_{0} \quad$ Wavenumber of free space $\left(\mathrm{m}^{-1}\right)$

$\lambda \quad$ Wavelength of light $(\mathrm{m})$

$\mu \quad$ Magnetic permeability $\left(\mathrm{H} \cdot \mathrm{m}^{-1}\right)$

$\mu_{0} \quad$ Magnetic permeability of free space $\left(\mathrm{H} \cdot \mathrm{m}^{-1}\right)$

$\mu_{\mathrm{a}} \quad$ Absorption coefficient $\left(\mathrm{m}^{-1}\right)$ 
$\mu_{\mathrm{s}} \quad$ Scattering coefficient $\left(\mathrm{m}^{-1}\right)$

$\omega \quad$ Wave's angular frequency $\left(\mathrm{rad} \cdot \mathrm{s}^{-1}\right)$

$\omega_{\mathrm{p}} \quad$ Plasma frequency of electron $\operatorname{gas}\left(\mathrm{rad} \cdot \mathrm{s}^{-1}\right)$

$\rho \quad$ Total charge density $\left(\mathrm{C} \cdot \mathrm{m}^{-3}\right)$

$\rho_{\mathrm{n}} \quad$ Number density $\left(\mathrm{m}^{-3}\right)$

$\sigma \quad$ Electrical conductivity $\left(\mathrm{S} \cdot \mathrm{m}^{-1}\right)$

$\sigma_{\text {abs }} \quad$ Absorption cross-section $\left(\mathrm{m}^{2}\right)$

$\sigma_{\text {ext }} \quad$ Extinction cross-section $\left(\mathrm{m}^{2}\right)$

$\sigma_{\text {sca }} \quad$ Scattering cross-section $\left(\mathrm{m}^{2}\right)$

$\tilde{\mu}_{\mathrm{r}} \quad$ Complex relative permeability (1)

$\tilde{\varepsilon_{\mathrm{r}}} \quad$ Complex relative permittivity (1)

$\tilde{n} \quad$ Complex index of refraction (1)

$\varepsilon \quad$ Electrical permittivity $\left(\mathrm{F} \cdot \mathrm{m}^{-1}\right)$

$\varepsilon_{0} \quad$ Electrical permittivity of free space $\left(\mathrm{F} \cdot \mathrm{m}^{-1}\right)$

$c_{0} \quad$ Speed of light in free space $(\mathrm{m})$

e Electron's charge $(\mathrm{C})$

$i \quad$ Imaginary number, defined as $\sqrt{-1}$

$I_{0} \quad$ Intensity of incident light (W)

$k \quad$ Extinction coefficient (1)

$l_{\infty} \quad$ Bulk mean free path $(\mathrm{m})$

$m_{\mathrm{e}} \quad$ Free electron's mass $(\mathrm{kg})$ 
$n \quad$ Real part of the complex index of refraction (1)

$v_{\mathrm{F}} \quad$ Fermi velocity $\left(\mathrm{m} \cdot \mathrm{s}^{-1}\right)$ 


\section{Chapter 1}

\section{Introduction}
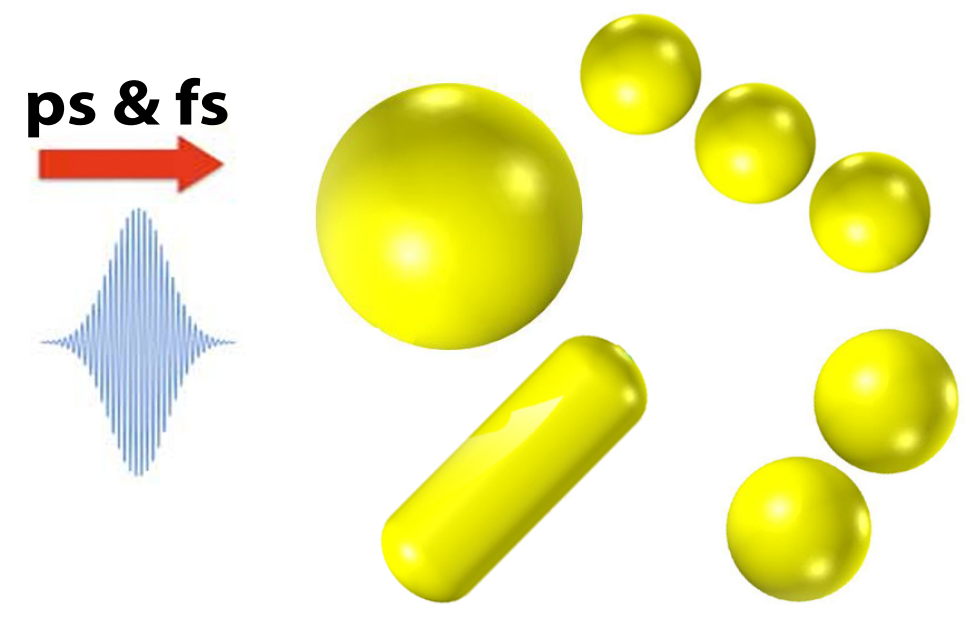

In this chapter an overview of the nanoparticle-mediated optical breakdown mechanism will be given, and it will be followed by a motivation and the specific aims that the author pursued in this thesis.

\subsection{Nanoparticle-mediated optical breakdown and its applications}

In 1963, Meyerand and Haught [1] reported the breakdown of gas at optical frequencies, which has been actively studied since [2 [5]. The laser induced breakdown (LIB) phenomena is the process of free electron plasma generation during interaction of a dielectric material with the strong field of a laser pulse. Strong field ionization of the optically transparent material makes it an electrical conductor. When the density of free electrons reaches the critical value of $10^{18}-10^{21} \mathrm{~cm}^{-3}$, plasma strongly absorb laser pulse energy so that the temperature of the plasma increases drastically and leads to rapid expansion and destruction of the material. Initially this phenomenon was viewed as an undesirable process that causes severe problems during laser machining. Nevertheless, with an improved understanding of the process and the physics behind it, optical breakdown has become a valuable tool in such applications as laser-induced breakdown spectroscopy [6], laser machining of transparent media such as glasses or polymers [7, 8], and manipulation of biological samples [9].

Plasmonic nanoparticles, such as gold nanorods, can enhance the application of optical 
breakdown. One such application is optoporation. In optoporation a laser pulse interacts with the cell membrane to form a pore caused by the optical breakdown of the medium [10, 11]. The exogenous molecules and nucleic acids can enter the cell through the osmotic pressure difference between the medium and the cytosol. The disadvantages of optoporation (without nanoparticles) are the high cost of a laser system, the inability to perform optoporation on multiple cells at a time, and the need for a precise alignment of the laser beam to the cell membrane. These disadvantages can be eliminated by the addition of plasmonic nanoparticles that are actively used in biomedical applications such as plasmonic photo-thermal therapy [12 15], cell detection and imaging [16 18]. The nanoparticles can target specific cells and act as a localized nano absorbers of the laser energy so that instead of alignment of the laser to a specific cell, a broad illumination can be used and the optoporation will be induced only at the localization of the nanoparticles.

LIB in the vicinity of gold nanoparticles is an area of an active research. In addition to optoporation, it is being studied for applications in many areas in medicine and biology, such as nanoparticle enhanced LIB spectroscopy [19, 20], cell nanosurgery [21 23] and drug release [24]. Such a broad range of applications stems from the gold nanoparticles' unique optical properties in the visible and near-infrared (NIR) wavelengths, where biological tissues are most transparent to incoming laser irradiation. Highly localized mechanical [16], thermal [13] or optical effects [25] can be achieved due to the nanoscale localization of the light that is cause by a gold nanoparticle. The interaction of light with a gold nanoparticle induces a localized surface plasmon resonance (LSPR), where the oscillation of quasi-free electrons along the gold nanoparticle's surface produces a near-field enhancement of the incident radiation. The spectral characteristics of LSPR are highly dependent on the nanoparticle morphology (shape/size/composition), aggregation, dielectric properties of the external environment [26].

An optical breakdown threshold is the laser irradiance needed to cause the LIB and is often used to characterize optical breakdown. Experimentally, optical breakdown in liquids is observed through luminescence or bubble formation. Theoretically, optical breakdown is usually defined as heating by the plasma to the boiling temperature of the medium or reaching a critical electron density in the medium, which is on the order of $\rho_{\text {crit }} \approx 10^{18}-10^{21} \mathrm{~cm}^{-3}[27$.

Due to the computational nature of this thesis, the optical breakdown threshold will be refered to the laser irradiance needed in order to generate a critical electron density 
up to $\rho_{\text {crit }} \approx 10^{20}-10^{21} \mathrm{~cm}^{-3}[27$. The optical breakdown threshold is a function of the electric field strength, laser pulse duration and wavelength. In nanoparticle-mediated LIB the electric field in the vicinity of the nanoparticle is enhanced due to their LSPR [22]. Nanoparticles can enhance optical breakdown using nanosecond (ns) [10, 28 30], picosecond (ps) [31 33] and femtosecond (fs) [34 36] laser pulses. Moreover, one can choose from a large variety of plasmonic nanoparticles available today to achieve different electric field enhancements in the vicinity of the particle at various wavelengths, allowing for modulation of the optical breakdown threshold. Therefore, a combination of excitation wavelength and nanoparticles morphology will determine the optical breakdown threshold.

Nanoparticle-mediated optical breakdown is a complex process due to the large variety of parameters involved, such as laser source characteristics (fluence/wavelength/pulse duration) and nanoparticle morphology (size/shape/composition). Therefore, the optimization and control of a nanoparticle-mediated optical breakdown threshold is challenging. Computational analysis can be used to model the optical breakdown process and to obtain insight into the LIB process. Although the computational modelling of nanoparticle-mediated LIB is difficult, there are many unanswered questions, such as how the optical properties of the nanoparticle are influenced by its local environment, and how the threshold of LIB depends on morphology of the nanoparticle and laser parameters, that motivate the development of numerical models.

\subsection{The mechanism of nanoparticle-mediated optical breakdown}

The mechanism of nanoparticle-mediated optical breakdown is multiscale in nature. The multiscale nature of the LIB phenomena in space and time is governed by the interaction of the laser light with the nanoparticle that occurs at the nanometer $(1-100 \mathrm{~nm})$, while the generated phenomena (heating, bubble formation, optical breakdown) occurs at micrometre scale. Furthermore, the time scale of pulse-nanopartcle interaction can be as small as tens of femtoseconds, while bubble nucleation and growth (which is what is detected experimentally) can take up to several nanoseconds. 


\subsubsection{Optical behaviour of nanoparticles}

Knowledge of optical response by a single nanoparticle is of high importance where the interaction of a laser pulse with the single nanoparticle initiates an optical breakdown in a cellular environment [32]. The understanding of optical properties of a single nanoparticle is hindered by unusual quantum mechanical effects, such as LSPR. This makes the optical behaviour of a nanoparticle quite different compared to the bulk optical properties of the material. When the size of a nanoparticle is much smaller than the wavelength of incident light, quantum effects arise due to the high surface-to-volume ratio of the density of atoms in the nanoparticle.

It is hard to determine the LSPR and optical properties (resonance wavelength and the LSPR line width) of an individual nanoparticle, experimentally or numerically. The spectral characteristics of LSPR are extremely sensitive to a nanoparticle's morphology (shape/size/composition) [37-40], aggregation [41] and local environment [38]. The LSPR is affected by the intrinsic size effects of the dielectric function of a nanoparticle [42, 43].

The complex dielectric function of a nanoparticle that affects the LSPR line width and plasmon resonance position, depends on the bulk damping coefficient, $\Gamma_{\text {bulk }}=\Gamma_{\mathrm{e}-\mathrm{ph}}+\Gamma_{\mathrm{e}-\mathrm{e}}$, which is a combination of electron-phonon scattering and electron-electron scattering; the damping coefficient due to electron-surface scattering, $\Gamma_{\text {surf }}$; and the radiation damping coefficient, $\Gamma_{\text {rad }}$ [42 44]. Precise and detailed knowledge of the damping processes is necessary for the effective optimization of the optical properties of plasmonic nanoparticles, for their efficient use in field enhancement-based applications such as nanoparticle-mediated LIB, nanostructure fabrication [45], cell nanosurgery [22], and a laser induced breakdown spectroscopy [19, 20]. Determination of the damping mechanism and its quantification in a colloidal solution is hampered by the broad shape and size distributions of nanoparticles resulting in an in-homogeneous line broadening. The single-particle extinction spectroscopy such as spatial modulation spectroscopy (SMS), can overcome this drawback [40, 43]. SMS measures the absolute extinction cross-section of the nanoparticle and, in combination with transmission electron microscopy (TEM), can also correlate the dimension of the nanoparticle being examined to its extinction cross-section. SMS is preferable over scattering-based methods since the absorption cross-section of a nanoparticle is much higher than the scattering cross-section and the size of a nanoparticle is below $20 \mathrm{~nm}$ in diameter [46, 47]. 
SMS, TEM and numerical analysis are together a powerful combination for the quantitative determination of the optical properties of a single nanoparticle. Gold nanorods are widely used in biological applications [48 50] due to tunability of the longitudinal plasmon resonance in the visible and NIR wavelengths [49, 51]. On the other hand, the absorption cross-section of a gold nanorod (GNR) depends on its size. For a smaller GNR, the absorption cross-section is higher than the scattering cross-section. The tunability of a plasmon resonance and a large absorption cross-section makes GNRs highly attractive for single particle absorption spectroscopy measurements and studies of line widths of LSPR [52].

\subsubsection{The process of nanoparticle-mediated optical breakdown in water}

Nanoparticle-mediated LIB is a non-linear laser energy absorption process that produces a low-density plasma in the surrounding region of the nanoparticle. Plasmonic nanoparticles, such as gold nanospheres and nanorods, enhance the electromagnetic field of light and strongly absorb the light. LIB is usually induced when the laser irradiance surpasses a certain threshold (which will henceforth be referred to as the optical breakdown threshold, $\left.I_{\mathrm{th}}\right)$. At this threshold, the free electron density in the medium exceeds a critical value, which has been found to be in the range of $10^{18}-10^{21} \mathrm{~cm}^{-3}$ [5, 27, 53 [57]. The interaction of a strong electromagnetic field with a gold nanoparticle in an aqueous media (which models a biological environment) can initiate breakdown either through multiphoton absorption and the tunneling effect (usually referred as a laser induced optical breakdown (LIOB)) [57, 58]; through a thermal initiation pathway (also known as laser induced thermal breakdown (LITB)) [59, 60]; or through the photo-thermal emission (PTE) of hot electrons off the nanoparticle surface [61, 62]. After some seed electrons have been generated via a combination of the processes mentioned above, the plasma starts to gain sufficient kinetic energy from the laser pulse by inverse Bremsstrahlung absorption (IBA) and grows through impact ionization, known as electron avalanche 63. LIB induces breakage of molecular and atomic bonds and will be accompanied by luminescence, cavitation, bubble and shock wave formation [55, 64.

In an aqueous medium without nanoparticles, the initiation of multiphoton absorption for LIOB will require between six and twelve photons with the same polarization to 
exceed the band-gap energy of water, which is $\sim 6.5 \mathrm{eV}$ [27]. LIOB occurs when the optical breakdown threshold is in the range $\sim 10^{11}-10^{13} \mathrm{~W} / \mathrm{cm}^{2}$ is surpassed in the focal region of the laser beam [27, 65]. For nanosecond pulses [59] or ultrashort pulse sequences separated by times in the order of the free electron energy thermalization time [66], LITB is a significant source of seed electrons, $\rho_{\text {seed }}$ (which is required to initiate the avalanche ionization (AI) [59]), when temperature of the medium increases above approximately $5000 \mathrm{~K}$. LITB starts with linear absorption and thermal ionization (TI) of the aqueous medium and continues with non-linear AI. The introduction of gold nanoparticles into an aqueous medium will lower the LIB threshold and provide an additional source of seed electrons form a PTE of hot electrons off the nanoparticle surface [62, 67]. During nanoparticle-mediated LIOB with pulses $<10$ ps the nanoparticle's lattice temperature is kept below the melting point $1337 \mathrm{~K}$ (for gold nanoparticles with diameter above $10 \mathrm{~nm}$ [68]) since the photon energy at this time-scale is transferred to the electrons in the gold nanoparticle and diffusion is minimized [69, 70]. The gold nanoparticles therefore retain their optical properties during LIB with pulses $<10 \mathrm{ps}$. Compared to femtosecond lasers, ultrashort picosecond lasers (with pulse duration between 1 to $10 \mathrm{ps}$ ) are beneficial due to their cheaper operational cost, ease of maintenance and higher power specifications. Such

ultrashort pulses (few picoseconds) can be achieved using Nd:YAG and Nd:YVO4 crystals in passively modulated mode-locked lasers, that are widely used in micromachining [71].

\subsubsection{The wavelength dependence of band structure of water}

Nanoparticle-mediated LIOB depends on the ionization energy, the level of impurities in the medium, nanoparticle morphology and concentration, and the laser beam properties (pulse duration, intensity and wavelength) [22]. While the electric field enhancement in the vicinity of a nanoparticle is dependent on both morphology of the particle and the wavelength of irradiation, the optical breakdown threshold, $I_{\text {th }}$, in pure water (as a model for biological media [72]) is also wavelength dependent [73 75]. Linz et al. [74] investigated the wavelength dependence of $I_{\text {th }}$ for nanosecond (ns) and femtosecond (fs) LIOB in pure water [73 76]. The study demonstrated, both experimentally and theoretically, the need for a correction to the band gap structure of water and an introduction of an additional ionization path. This was in conflict with prior models of water as an amorphous semiconductor [77] with an energy gap between the valence and the conduction bands 
of $6.5 \mathrm{eV}$ [53]. The need for corrections to the band structure of water became obvious when it was found that the direct ionization of an electron from the valence band (VB) to the conduction band (CB) occurs only with an excitation photon, $E_{\mathrm{ex}} \geq 9.5 \mathrm{eV}[78,79]$, and that the band gap of $6.5 \mathrm{eV}$ is really the minimum energy (initiation energy, $E_{\text {ini }}$ ) for direct ionization of the valence electron into an intermediate solvated state [80]. That implies that in the visible and near-infrared wavelengths, the breakdown initiation occurs only as a two-step process: first, by multiphoton absorption of valence band electrons into a solvated state $\left(\tilde{A} 1{ }^{1} B_{1}\right)$ and then by upconversion into the $\mathrm{CB}$ via multiphoton absorption or by stepwise excitation through intermediate $p$ states [74, 81]. Linz et al. [74] showed that these processes can be modelled by selecting $E_{\text {gap }}=9.5 \mathrm{eV}$ (which will adequately account for both, vertical $(11 \mathrm{eV})$ and autoionization $(9.5 \mathrm{eV})$ processes in water) and $E_{\text {ini }}=-0.0012 \lambda(\mathrm{eV} / \mathrm{nm})+7.58(\mathrm{eV})$ (for ionization through intermediate solvated state). It was also found that $\rho_{\text {seed }}$ needed to initiate AI had wavelength dependence [74], as well. By fitting a theoretical calculation to experimental data Linz et al. [74] obtained $\rho_{\text {seed }}=\left(1 \mathrm{~cm}^{-3}\right) 10^{-0.01116\left(\mathrm{~nm}^{-1}\right) \lambda+23.5}$.

It is expected that an intermediate initiation channel [74] should not affect $I_{\text {th }}$ during nanoparticle-mediated LIOB with long pulses since the seed electron generation will be dominated by the PTE of hot electrons off the nanoparticle surface [61] (the absorption regime). However, as the pulse duration decreases, multiphoton absorption and tunnel ionization start to dominate seed electron generation (the near-field regime). As such, nanoparticle-mediated LIOB should become wavelength dependent due to the wavelength dependence of $\rho_{\text {seed }}$ and a lower $E_{\text {ini }}$ wavelength dependence. To the best of my knowledge, there has been no study of the wavelength dependence of nanoparticle-mediated LIOB in water.

\subsection{Motivation}

\subsubsection{Modelling laser induced optical breakdown (LIOB)}

The study and understanding of a nanoparticle-mediated LIOB process is complicated due to its non-linear nature. In such cases, a computational analysis becomes a cost and time effective tool that can uncover new insights into the LIOB phenomena. For example, Boulais et al. 22] developed a complex model of the nanoparticle-mediated 
LIOB process for a femtosecond pulse exposure of a GNR. The theoretical analysis by Boulais et al. 67] revealed the existence of two different physical regimes of a free electron plasma generation in the vicinity of a GNR during ultrafast pulse exposure. For a fluence lower than $3 \mathrm{~mJ} / \mathrm{cm}^{2}$, the gold nanorod strongly absorbed the incident pulse energy and the majority of the seed electrons were produced by PTE (the absorption regime), while for fluences higher than $3 \mathrm{~mJ} / \mathrm{cm}^{2}$ the free electron plasma formation was dominated by multiphoton absorption due to a high near-field enhancement surrounding the GNR (the near-field regime).

Although several theoretical papers have been published in recent years [67, 82, 83], there are areas where the gaps in the understanding of a nanoparticle-mediated LIOB still exists. For example, the lack of a detailed theoretical explanation of mechanism of plasma formation in the vicinity of gold nanoparticles in a picosecond and nanosecond regime hinders the understanding of experimental results of gold nanoparticle mediated cell transfection [10, 29, 32, 84]. For such an application as transfection, a complete theoretical picture of a plasma formation in a picosecond and nanosecond regime with the use of gold nanoparticles is needed. This will help in the optimization process [84 and will lead towards a lowering of the cost of a transfection operation, in comparison to utilization of femtosecond lasers. The lack of knowledge and understanding of how morphology and assembly of gold nanoparticles plays a role in optical breakdown threshold lowering, complicates the design and optimization of smart agents for biological applications of gold nanoparticles in LIOB-related technologies, where gold clusters can target only specific species based on the chemical and physical conditions of the surroundings [85, 86].

\subsubsection{The current state of electromagnetic (EM) modelling}

Optical properties of gold nanoparticles can be characterized by empirical and theoretical methods. The experimental approach consists of spectroscopic measurements of a colloidal solution [87] or individual nanoparticles [46, 88], while the theoretical approach involves the analytical solution of the Mie [89] or Rayleigh of light propagation through the medium for spherical objects or numerical solution of the Maxwell's equations for arbitrary shaped objects. The optical response of the single nanoparticle is highly sensitive to the particle's morphology [40]. This high sensitivity is the result of the confinement of free electrons and plasmon surface resonance effects. The later factors complicate 
the comparison of the theoretical models to experimentally measured optical properties of single and colloidal nanoparticles. Such comparisons are only possible by combining individual particle spectroscopy with electron microscopy of the same particle, and theoretical modelling based on the known nanoparticle's morphology from the experimental data [90, 91]. To obtain a good quantitative comparison against experimental data, size-dependent corrections to the bulk dielectric function [92] of the metal nanoparticle are needed. These corrections determine the line widths of the surface plasmon resonance and illustrate the competition between radiation damping (for large particles the radiation damping cause an increase in the line width) and surface scattering (for small particles the plasmon resonance is broadened by surface scattering). The size-dependent corrections to bulk dielectric function usually appear as fitting parameters in a numerical model [88].

Computational electromagnetic methods (CEM) are numerical techniques that are widely used to model optical behaviour of plasmonic nanoparticles [93]. The Maxwell's equations are usually solved by CEM to model the light propagation, scattering and attenuation, through the medium or an object. The finite element method (FEM) is a numerical technique that finds an approximate solution to a partial differential equation (PDE) by decomposing space into small regions (elements) and equations into a system of simpler equations. This method is widely used in electromagnetic (EM) to describe the behaviour of the electromagnetic wave incident on an object [94 96]. FEM has shown excellent agreement against spectroscopic studies on a single gold nanoparticle [40, 88] by incorporating experimental conditions and nanoparticle's morphology into the model [40]. An excellent agreement between theory and experiment provides a justification of the size-dependent corrections to the bulk dielectric function of gold [46, 92] and supports further numerical analysis of the optical properties and plasmon coupling effects in the gold nanoparticle assemblies.

\subsubsection{The current state of LIOB modelling}

To model low-density plasma generation in the vicinity of a gold nanoparticle by a laser pulse, one should account for several physical phenomena that occur during the pulse, such as: (1) electromagnetic field interaction with gold nanoparticles and their environment, (2) absorption of the pulse energy and heating by the nanoparticle and their 
environment, and (3) free electron plasma generation and its effect on the optical properties of the environment.

Several theoretical models have been published in recent years to address the theoretical description of a nanoparticle-mediated LIOB. To highlight some, Bisker and Yelin [82 published a theoretical analysis of a short pulse interaction with silver and gold spherical nanoparticles. Although in their model the authors have varied pulse duration, size and composition of the particle, their theoretical description is lacking two-way coupling between phenomena involved in a free electron plasma generation; only applicable to spherical nanoparticles; missing description of PTE and TI as a source of the seed electron generation; and simplify treatment of water as an amorphous semiconductor with a band gap, $E_{\text {gap }}=6.5 \mathrm{eV}$, rather than using the wavelength dependent $E_{\text {gap }}(\lambda)$ [59, 74]. The model by Boulais et al. [62] is more complete and includes a two-way coupling between physical phenomena involved, can be used for the arbitrary shaped nanoparticles and was used for femtosecond pulse-particle interaction. The weak points of the model come in its in applicability for long pulses ( $>1 \mathrm{ps}$ ) or ultrashort pulse sequences [59, 66], where thermal ionization of the water becomes important and in the use of a simplified treatment of the water's band structure. Also Boulais et al. excluded size corrections to the dielectric function of a gold nanoparticle [26] which limits results to nanoparticles with a diameter bigger than $20 \mathrm{~nm}$, while for smaller nanoparticles, the optical properties will vary a lot from the bulk [44]. Also this model was used to model LIOB of a single gold nanoparticle while nanoparticle ensembles become highly popular in active targeting applications, so that the understanding of how an assembly of nanoparticles will affect an optical breakdown threshold is needed. Nevertheless, the theoretical analysis made by Boulais et al. [22], helped to predict the existence of two regimes of a nanoparticle-mediated LIOB in water - absorption and near-field regimes, where depending on the intensity of a laser pulse, one can generate free electron plasma via either PTE (absorption regime, low power of the laser) or multiphoton ionization (MPI) (near-field regime, high power of the pulse) 67]. Another model that needs to be highlighted is published by Hatef and Meunier [83. Hatef and Meunier modelled an interaction of an off-resonance femtosecond pulse with a monomer and dimers of a gold nanosphere. This study demonstrated how the inter-particle distance affects the laser energy deposition to the generated free electron plasma with nanoparticle dimers. The authors omitted PTE and TI in their plasma generation code, and once again used a sim- 
plified picture of water's band structure and in addition, no corrections to bulk dielectric function of gold were used.

As it will be shown in Chapter 4 , for a complete picture of a free electron production in the vicinity of a nanoparticle, photo-thermal emission must be considered as a significant source of seed electrons, even for ultrafast fs pulses. Another attempt at providing guidance for a rational design of plasmonic nanoparticles for an enhanced cavitation and a cell perforation was published recently by Lachaine et al. [97]. In this work, Lachaine et al. developed a methodology that provided a general strategy for a systematic design of nanoparticles for applications that utilize a LIOB effect. The theoretical framework of the authors is rather simplistic, which leads to inclusion of many fitting assumptions in order to match experimental data, that were not properly justified. In some cases, fitting parameters were not enough to match experimental data. In their model, the authors used Mie theory to calculate optical properties of the gold nanospheres and nanoshells (dielectric core silica and gold shell), and a simplistic rate equation to calculate plasma formation generated by MPI and AI.

A comparison table of what were included as the components into the computational models (showed as green) and excluded (red) components, can be seen in Table 1.1. All published models have several major weaknesses:

1. Oversimplification of the band structure of water. As a result, all models need to be updated in accordance with latest findings of $E_{\text {gap }}(\lambda)$ and $\rho_{\text {seed }}(\lambda)$ of water given by Linz et al. [59], Liang et al. [66], Linz et al. [74].

2. Another weak point of all published nanoparticle-mediated optical breakdown models is an omission of the thermal ionization, that is important for long pulses ( $>1 \mathrm{ps})$ and ultrashort pulse sequences [59, 66], especially when the optical breakdown threshold is set by the boiling temperature of the water rather than by a critical free electron density.

3. Most of the models (except for Boulais et al. [62, 67]) neglect photo-thermal emission which is an important source of seed electron production for any pulse duration.

4. Most of the models (except for Bisker and Yelin [82]) use the bulk dielectric function of gold, which is valid only for large particles (with diameter $>150 \mathrm{~nm}$ ). 


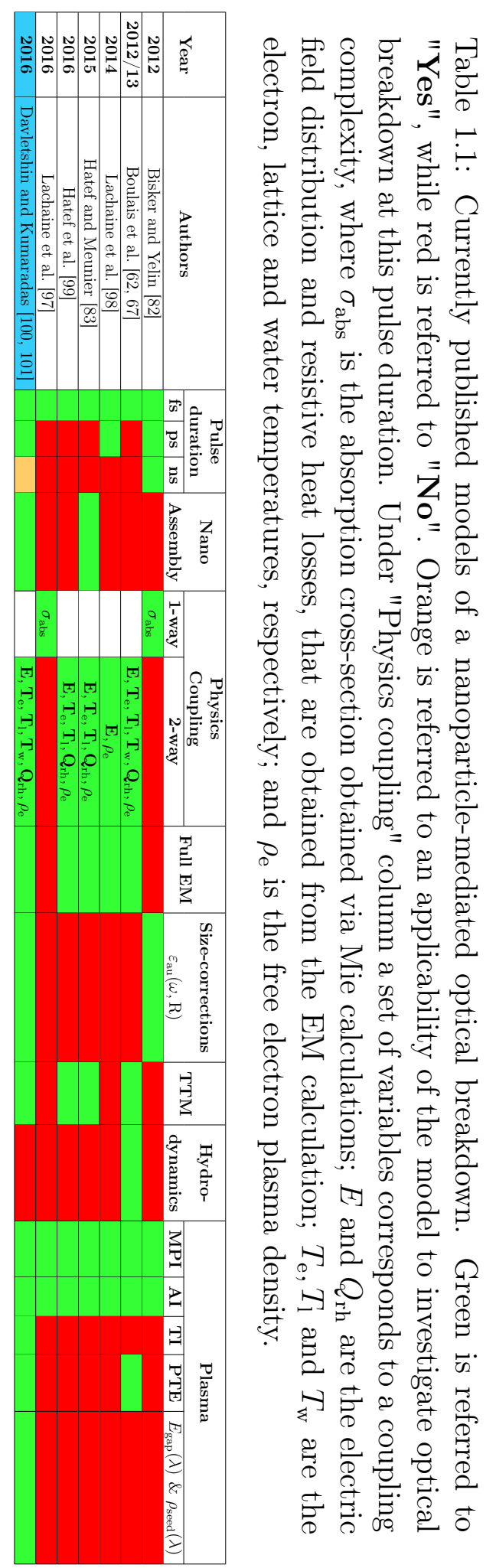


5. Only in one publication, by Hatef and Meunier [83], the authors looked at the plasmon coupling effect during LIOB. This was done for the same shape of nanoparticle with no analysis of how changes to the nanoparticle's morphology will affect LIOB.

6. Last, but not least, there was no rigorous analysis of the nanoparticle-mediated optical breakdown process with inclusion of all possible phenomena during picosecond and nanosecond pulse exposure or multiple pulse sequences for all pulse durations.

\subsubsection{Challenges in a computational analysis}

The main challenges of the computational analysis and optimization of the nanoparticlemediated LIOB phenomena come from:

1. The complexity of the optical characterization of a nanoparticle

2. The variety of laser exposure parameters

3. A strongly coupled physics of LIOB.

\subsubsection{Optical characterization of a nanoparticle}

Although a good degree of control in gold nanoparticles synthesis has been achieved, the high sensitivity of the optical response of such nanoparticles to their morphology makes it difficult to interpret the optical response measured on ensembles of nanoparticles and to compare the results to theoretical models. Such comparisons can only be done by investigating individual nanoparticles of known morphology and developing complete theoretical models that take detailed morphology and experimental conditions into account. This has been done by combining individual nanoparticle spectroscopy with TEM or scanning electron microscopy (SEM) [90, 91] and theoretical models. Such experimental and theoretical combinations can also be used to obtain some information on the morphology and intrinsic characteristics of individual nanoparticles and their environment [46, 88, 92, 102 104.

For small nanospheres $(<20 \mathrm{~nm}$ diameter $)$, the surface plasmon resonance (SPR) wavelength, $\lambda_{\mathrm{R}}$, is determined predominantly by the particle's environment, while the SPR extinction cross-section, $\sigma_{\text {ext }}\left(\lambda_{\mathrm{R}}\right)$, depends on both the particle size and the environment's refractive index [105, 106]. Therefore, single particle spectroscopy alone can 
be used to obtain information on the particle's environment, morphology, and intrinsic characteristics, in situ 103. A quasi-static approximation based on Mie theory has been predominantly used in this case [46, 103, 107], assuming a homogeneous surrounding with an effective index of refraction to represent the effect of the substrate (if present) and other heterogeneities in the particle's environment [90, 108].

For more complex morphologies, such as nanorods, the dependence of the relationship between the optical spectral features and the characteristics of a particle and its environment cannot be disentangled, making it difficult to fully characterize the particle geometry from optical measurements alone. Nanorods exhibit a dominant SPR known as the longitudinal SPR for light polarized along the major axis of the nanoparticle. This shows up as a strong and well-defined band in their extinction and scattering spectra [42, 88], centered at a wavelength that is sensitive to small modifications in their local environment [51, 109]. As compared to spheres, the characteristics of the resonance depend also on the shape and aspect ratio of the particle. If the particle's dielectric function and the environment's refractive index are known, then optical spectroscopy, combined with inverse modeling, can be used to infer the morphology of the nanoparticle (aspectratio and volume) [88, 108. Conversely, if the shape and size of the particle are known (using correlated imaging of the particle's morphology), then modeling can provide the refractive index of the environment or the metal's dielectric function, as discussed below. The most common theoretical models have been based on Mie-Gans theory (assuming spheroidal geometry for the particle), but also on numerical methods such as the discretedipole approximation (DDA), the boundary element method (BEM), the finite difference time domain (FDTD), and the FEM (FEM; for arbitrary shaped objects). The FEM allows for easy inclusion of arbitrary heterogeneities such as substrates and particle surface coatings while avoiding dense matrices and will be used here.

Although most investigations have been performed by dispersing the nanoparticles onto a transparent substrate, optical modeling has frequently assumed a homogeneous environment and used an effective refractive index, $n_{\text {sur }}$, as a parameter to describe it [88, 110. The optical response of chemically synthesized nanospheres deposited on a substrate has been observed to largely vary from particle to particle even within the same sample [102, 108. Single particle measurements on elongated nanoparticles have also shown these fluctuations [40]. The presence of embedding a polymer matrix has been shown to reduce the sensitivity of the optical properties of gold nanospheres and nanoprisms to 
the surrounding medium's refractive index [88, 111]. These findings raise the important question of the impact of the local environment (i.e., the substrate, surfactant molecules or residual solvent) on the measured optical properties of complex shaped nanoparticles.

\subsubsection{Laser source}

The pulse duration of the laser, whether it is ns or ps or fs, defines the appropriate physics behind laser pulse-nanostructure interaction [28, 112]. The correct set of equations is needed in order to accurately describe all processes in different timescales. The physical properties of the medium and nanoparticle are also wavelength dependent and must be accounted for in the modelling [59, 74].

\subsubsection{Strongly coupled multiphysics phenomena}

The EM wave interaction of a laser pulse with an electron structure of a medium and nanoparticle, initiates a strongly coupled multiphysics phenomena of the LIOB. This interaction leads to a temperature increase of the nanoparticles' electrons and lattice [28, 113], heat diffusion from the nanostructure to the surrounding medium, PTE of thermionic electrons off the nanostructure surface [61], ionization of the medium that includes the terms of a MPI [114], tunnel ionization, impact ionization [63], TI [59, 66], changes in the refractive indexes due to high density of free electron plasma, the free electron plasma collision losses with neutral molecules, and an electron recombination of ionized molecules [59], melting of the nanostructure, bubble formation and growth etc. All of the above mentioned phenomena presents a system of a strongly coupled events with inter-dependency. Such a system of events rapidly becomes a computationally expensive task to solve.

The gaps in the theoretical description of the nanoparticle-mediated optical breakdown process during fs, ps and ns pulse exposure motivated the development of a numerical model that addresses the current gaps in the theoretical description of the nanoparticle-mediated LIOB process, and provide some new insights into the phenomena. The conducted study of nanoparticle-mediated optical breakdown phenomena was done to help with the development of LIOB-related fields, to advance the understanding of nanoparticle-laser interactions, and to improve design of current experiments by accounting for all related optical and thermal effects. 


\subsection{Thesis objective and specific aims}

The objective of the thesis is to develop a fully coupled model of nanoparticle-mediated optical breakdown in water, that includes the size-dependent dielectric function of gold, the morphology of the particle, heat transfer in gold nanoparticle and water, a plasma model that includes MPI, AI, PTE and TI and the wavelength dependence of the band gap.

The specific aims of the research and this thesis were to:

1. Develop a finite element model of a LIOB in water caused by gold nanoparticles interaction with laser pulse

2. Test the model against experimental results

3. Use the model to study the role of environment on optical properties of a single gold nanoparticle

4. Use the LIOB model to study the role of morphology and plasmon coupling on nanoparticle-mediated optical breakdown in water

5. Use the LIOB model to study the role of wavelength dependent properties of a nanoparticle and water on optical breakdown threshold

\subsection{Thesis organization}

Chapter 2 explains the theory of a laser-nanoparticle interaction, including optical properties of the gold nanoparticle, heat transfer in the nanoparticle and surroundings, and free electron plasma generation.

Chapter 3 provides an overview of the model and the analysis used is the thesis.

Chapter 4 presents the results of a theoretical analysis of:

- a single silica coated and uncoated gold nanorod interaction with the light including an experimental quantitative validation that was published in the ACS Nano journal (2012) by Davletshin et al. [26]; 
- a nanoparticle-mediated LIOB process for picosecond pulses. The model was used to understand the role of morphology on the lowering of the optical breakdown threshold through the study of a 6 ps pulse interaction at in- and off-resonance wavelengths with a gold nanorod monomer, and a gold nanosphere monomer, dimer and trimer. The findings were published in the Beilstein Journal of Nanotechnology (2016) by Davletshin and Kumaradas [100].

- a study of the wavelength dependence of the band gap structure of the water during nanoparticle-mediated LIOB. In this study the wavelength dependence of a gold nanorod-mediated LIOB threshold during infrared exposures to ultrashort picosecond and femtosecond pulses was analyzed. The findings were submitted to the Annalen der Physik journal (2016) by Davletshin and Kumaradas [101].

- and the results of a nanoparticle-mediated optical breakdown model validation against published experimental data by Lachaine et al. [97]. The findings of the validation were prepared for publication in Optics Express (2016).

Chapter 5 highlights the significance and conclusions of the work that has been conducted.

Chapter 6 provides a discussion of the optical breakdown definition through plasma electron density and temperature and provides recommendations for future work. 



\section{Chapter 2}

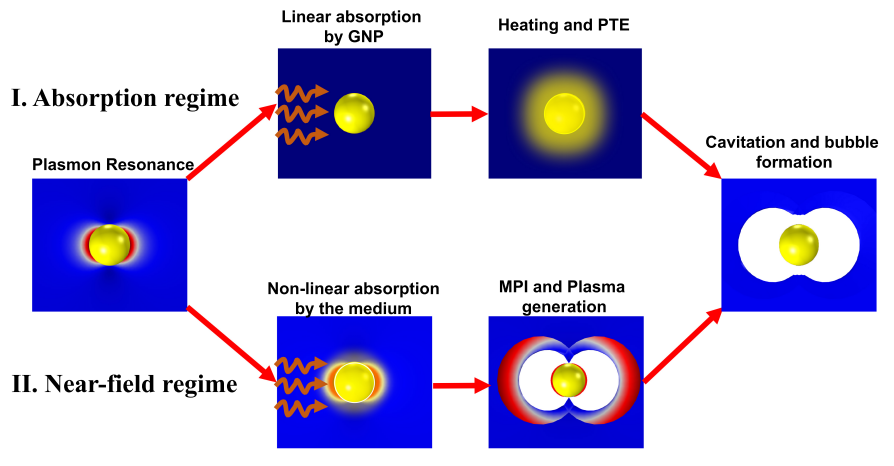

\section{Theory}

The understanding of the gold nanoparticle optical response to the incident laser pulse and optical breakdown requires the understanding of the physics of surface plasmon resonance and free electron plasma generation in the vicinity of colloidal gold particles. The theory and basic concepts of these disciplines will be introduced in this chapter. It will include classical electrodynamics for the description of the optical properties of nanoparticle, a two temperature model for the description of nanoparticle heating due to interaction with the laser pulse, and a strong field ionization of the water for the description of free electron plasma formation.

\subsection{The electromagnetic model}

The phenomena of LSPR can be described via computational electrodynamics, where one can solve macroscopic Maxwell's equations. It can be shown that in a nonmagnetic medium and in the absence of external charges the solution for the electric field, E, can be obtained from

$$
\nabla\left(\frac{1}{\tilde{\varepsilon}} \mathbf{E} \cdot \nabla \tilde{\varepsilon}\right)+\nabla \times \nabla \times \mathbf{E}=\mu_{0} \varepsilon_{0} \tilde{\varepsilon} \frac{\partial^{2} \mathbf{E}}{\partial t^{2}},
$$

where $\mu_{0}, \varepsilon_{0}$ and $\tilde{\varepsilon}$ are magnetic permeability of free space, electrical permittivity of free space and complex relative electrical permittivity, respectively. Assuming that the electric field is time harmonic $\mathbf{E}(\mathbf{r}, t)=\mathbf{E}(\mathbf{r}) \mathrm{e}^{-i \omega t}$, where $\omega$ is the wave's angular frequency, and that the spatial variation of the $\tilde{\varepsilon}$ is negligible, equation 2.1 then takes form of the 
Helmholtz wave equation:

$$
\nabla \times \nabla \times \mathbf{E}-\kappa_{0}^{2} \tilde{\varepsilon} \mathbf{E}=\mathbf{0}
$$

where $\kappa_{0}=\omega \sqrt{\varepsilon_{0} \mu_{0}}$ is a wave number in vacuum. Solution of equation 2.2 with appropriate boundary conditions provides the spatial distribution of the electromagnetic field in the presence of a plasmonic nanoparticle and can predict phenomena such as LSPR.

\subsubsection{Size corrections to bulk dielectric function}

Two most popular/standard shapes of the gold nanoparticles that are used in biology and medicine research and therefore studied in this thesis, are nanospheres and nanorods. The electric field enhancement efficiency and the plasmon resonance position of these particles, as well as any other plasmon nanoparticles, will be determined by the shape and size of the nanoparticle. An electric field enhancement of up to 55 - 70 times [22] can be reached during a gold nanorod interaction with laser pulse at the plasmon resonance wavelength. Nanospheres can have field enhancement factor of around 5-7 (see Figure 2.1.).
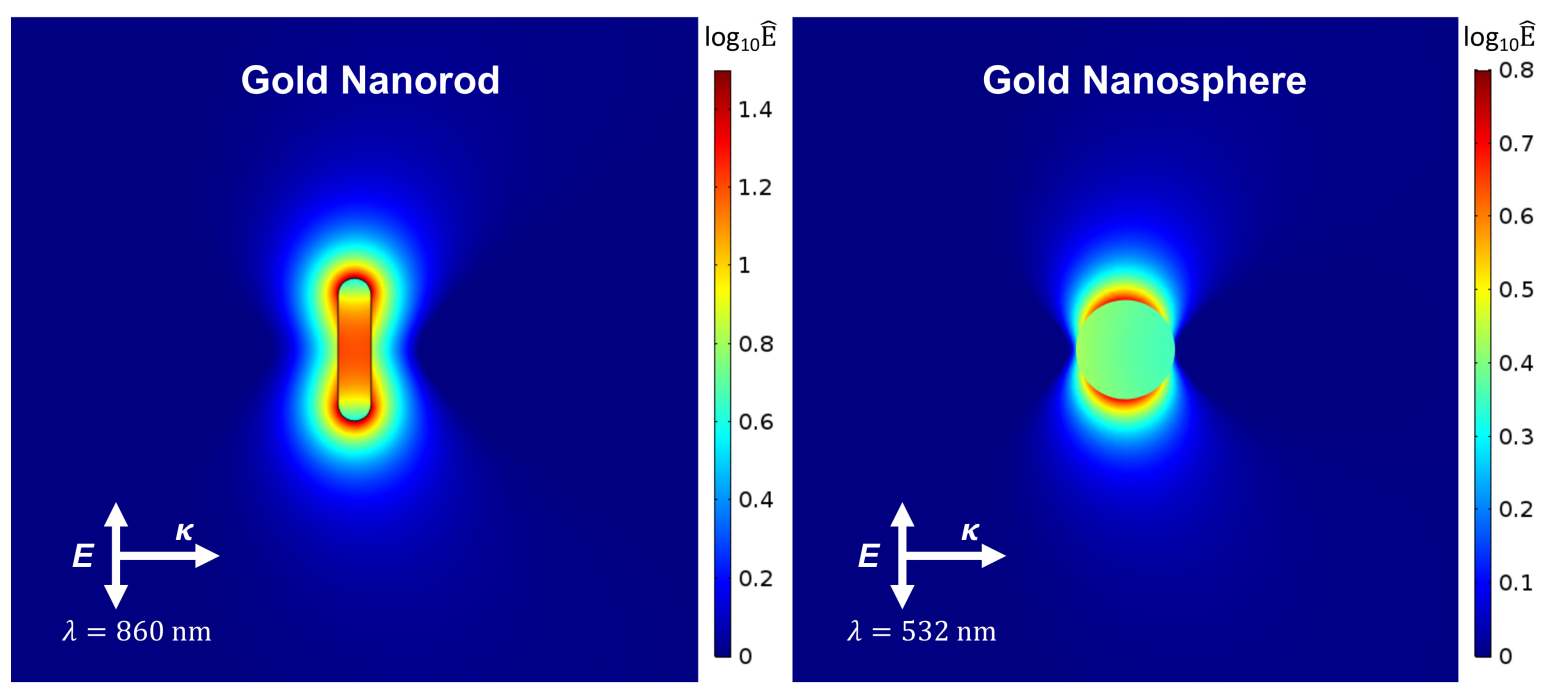

Figure 2.1: Plots of the relative electric field enhancement, $\hat{\mathrm{E}}$, for $10 \times 43 \mathrm{~nm}$ gold nanorod and $30 \mathrm{~nm}$ in diameter gold nanosphere, exposed at $860 \mathrm{~nm}$ and $532 \mathrm{~nm}$ wavelength, respectively. Relative electric field enhancement, $\hat{\mathrm{E}}=|\mathrm{E}| /\left|\mathrm{E}_{\text {inc }}\right|$, where $|\mathrm{E}|=\sqrt{\mathbf{E} \cdot \mathbf{E}^{*}}$ is the amplitude of the total electric field and $\left|\mathrm{E}_{\text {inc }}\right|$ is the amplitude of the incident field. $\hat{\mathrm{E}}$ is shown on a colour log-scale. 
Since the size of a nanoparticle plays an important role in their optical properties, it is crucial to correctly account for nanoparticle's morphology and the dielectric function of the nanoparticle during computational modelling. For noble metals, the dielectric function of a nanoparticle can be described by the free electron model with the contribution of interband transitions, that can not be neglected in the visible frequency range $(\lambda=400-700 \mathrm{~nm})$. Using the Drude-Lorenz-Sommerfeld model [44] and a contribution of interband transitions, the complex dielectric function can be written as a sum of susceptibilities $\tilde{\chi}^{\mathrm{IB}}$ and $\tilde{\chi}^{\mathrm{D}}$,

$$
\tilde{\varepsilon}_{\text {bulk }}(\omega)=1+\tilde{\chi}^{\mathrm{D}}+\tilde{\chi}^{\mathrm{IB}}=1-\frac{\omega_{p}^{2}}{\omega^{2}+i \Gamma \omega}+\tilde{\chi}^{\mathrm{IB}}
$$

where $\tilde{\chi}^{\mathrm{D}}$ is the free electron (Drude) susceptibility and $\tilde{\chi}^{\mathrm{IB}}$ is the interband transition susceptibility, $\Gamma$ is a phenomenological damping constant and $\omega_{p}$ is the plasma frequency ${ }^{*}$ of the free electron gas. The damping constant in the case of nanoparticles, is a sum of contributions due to electron interactions with phonons, electrons, lattice defects and impurities, which is denoted as $\Gamma_{\mathrm{bulk}}=v_{\mathrm{F}} / l_{\infty}$; electron collisions and scattering from the nanoparticle's surface which is denoted as $\Gamma_{\text {surf }}=A v_{\mathrm{F}} / L_{\text {eff }}$; and a radiation damping which is proportional to nanoparticle's volume and given by $\Gamma_{\mathrm{rad}}=\hbar \eta V_{\mathrm{np}} / \pi$, with $\eta=$ $(5.5 \pm 1,5) \times 10^{-7} \mathrm{fs}^{-1} \mathrm{~nm}^{-3}$ (averaged based on several experimental data of Novo et al. [92] and Sönnichsen et al. [46]). $l_{\infty}$ is the bulk mean free path of the electron between collisions, $v_{F}=1.41 \mathrm{~nm} / \mathrm{fs}$ is the Fermi velocity [44, $A$ is a dimensionless constant (called the broadening parameter) on the order of unity, and $L_{\mathrm{eff}}=4 V_{\mathrm{np}} / S_{\mathrm{np}}$ is the reduced effective mean free path length, where $V_{\mathrm{np}}$ and $S_{\mathrm{np}}$ are the volume an surface area of the arbitrary shaped nanoparticle, respectively.

By accounting for all intrinsic effects and interband transitions the complex dielectric function of a gold nanoparticle for a size of less than $150 \mathrm{~nm}$ in radius is

$$
\tilde{\varepsilon}\left(\omega, L_{\mathrm{eff}}\right)=\tilde{\varepsilon}_{\mathrm{bulk}}(\omega)+\frac{\omega_{p}^{2}}{\omega^{2}+i \omega \Gamma_{\mathrm{bulk}}}-\frac{\omega_{p}^{2}}{\omega^{2}+i \omega\left(\Gamma_{\mathrm{bulk}}+\frac{A v_{\mathrm{F}}}{L_{\mathrm{eff}}}+\frac{\hbar \eta V_{\mathrm{np}}}{\pi}\right)}
$$

where $\tilde{\varepsilon}_{\text {bulk }}(\omega)$ accounts for the interband transitions and usually taken from experimental

${ }^{*}$ For gold $\omega_{p}=\sqrt{\frac{\rho_{\mathrm{e}} e^{2}}{\varepsilon_{0} m_{e}}}=1.38 \cdot 10^{16} 1 / \mathrm{s}$, 44 
data of Johnson and Christy [115].

For gold nanoparticles with diameters greater than $40 \mathrm{~nm}$, the dielectric function approximate the bulk dielectric function, which can be obtained from the experimental data of Johnson and Christy [115].

\subsection{The two-temperature model (TTM) of a gold nanopar- ticle heating}

The laser excitation of conduction electrons in the nanoparticle via plasmon resonance is followed by plasmon resonance dephasing. Plasmon resonance dephasing occurs either through the intrinsic damping processes inside of the nanoparticle or electron surface scattering and photon absorption. Deposited energy excites electrons that spread out to different levels in the conduction band and rapidly equilibrate via an electron-electron scattering on a time scale of few 100 fs. Because of such short times, during a femtosecond pulse exposure the temperature of electrons can rapidly reach several thousand Kelvin. These hot electrons transfer their energy to the lattice of the nanoparticle through electron-phonon coupling within $\tau_{e-p h} \approx 10 \mathrm{ps}$ [28]. Finally, the energy dissipation to the environment occurs as a phonon-phonon scattering in the time-scale of $\sim 100$ ps.

For ultrafast picosecond and femtosecond laser-nanoparticle interaction, where pulse length is less or similar to the electron-phonon equilibrium time, $\tau_{e-p h}$, the heat transfer and coupling between electronic, $T_{\mathrm{e}}$ and lattice, $T_{1}$, temperatures can be described by a hyperbolic two-temperature model (TTM), which is based on quantum and statistical mechanics [116]:

$$
\begin{gathered}
C_{\mathrm{e}} \frac{\partial T_{\mathrm{e}}}{\partial t}+\nabla \cdot \boldsymbol{q}_{\mathrm{e}}=-G\left(T_{\mathrm{e}}-T_{\mathrm{l}}\right)+Q_{\mathrm{rh}} \\
\tau_{\mathrm{e}} \frac{\partial \boldsymbol{q}_{\mathrm{e}}}{\partial t}+\boldsymbol{q}_{\mathrm{e}}=-\kappa_{\mathrm{e}} \nabla T_{\mathrm{e}} \\
C_{\mathrm{l}} \frac{\partial T_{\mathrm{l}}}{\partial t}+\nabla \cdot \boldsymbol{q}_{\mathrm{l}}=G\left(T_{\mathrm{e}}-T_{\mathrm{l}}\right), \\
\tau_{\mathrm{l}} \partial \frac{\boldsymbol{q}_{\mathrm{l}}}{\partial t}+\boldsymbol{q}_{\mathrm{l}}=-\kappa_{\mathrm{l}} \nabla T_{\mathrm{l}} \\
Q_{\mathrm{au} \mid \mathrm{w}}=q_{0}\left(T_{1}-T_{\mathrm{w}}\right)
\end{gathered}
$$


where $T_{\mathrm{e}}$ and $T_{\mathrm{l}}$ are the electron and lattice temperatures, $C_{\mathrm{e}}$ and $C_{\mathrm{l}}$ are the electronic and lattice heat capacities, $\boldsymbol{q}_{\mathrm{e}}$ and $\boldsymbol{q}_{\mathrm{l}}$ are the electronic and lattice heat flux vectors, $G$ is the electron-phonon coupling factor, $\tau_{\mathrm{e}}$ and $\tau_{1}$ are the electron and ion collision times, $\kappa_{\mathrm{e}}$ and $\kappa_{\mathrm{l}}$ are the electron and lattice thermal conductivity, $T_{\mathrm{w}}$ is the temperature of the surrounding medium, and $q_{0}$ is the thermal conductance at gold-water interface (given by Plech et al. [68]), respectively.

In equations 2.5 2.9 the speed of heat propagation in space is considerate to be finite, while the temperature difference between the electrons and the lattice is linked by the electron-phonon coupling factor $G$.

When an electric field is interacting with a gold nanoparticle, resistive losses occurs. These losses, $Q_{\mathrm{rh}}$ [117], can be calculated by electromagnetic theory via:

$$
Q_{\mathrm{rh}}=\frac{1}{2} \Re\left[(\sigma-j \omega \varepsilon) \mathbf{E} \cdot \mathbf{E}^{*}\right],
$$

where $\Re$ is the real component, and $\sigma$ is the electric conductivity, respectively. Resistive losses during laser pulse interaction are used in the two-temperature model (TTM) (see equation 2.5) as a link to electromagnetic model (eq 2.2). Furthermore, heat diffusion from the gold lattice to the surrounding medium is accounted through interface conductance, $Q_{\mathrm{au} \mid \mathrm{w}}[118$. Interface conductance is related to a temperature drop at an interface of a nanoparticle where a heat flux crossing the interface. This is used as a coupling parameter between a heat transfer in the nanoparticle (TTM) and the surrounding medium. The effect of nanoparticle's size to the heat diffusion mechanism was not accounted for in our simulations [28]. The effect can be neglected pulse durations studied, but must be accounted for with longer pulses [118]. It should be also noted that the heat transfer at gold-water interface will be highly dependent on the molecular coating of the nanoparticle [119, 120]. The effect of various molecular coatings on heat transfer at the surface is difficult to characterize and was therefore not accounted for in this study. $T_{\mathrm{e}}$ was used as a coupling parameter between the TTM and Plasma model.

Further details on the TTM and input parameters can be found in the publications by Chen et al. [121] and Ekici et al. [118] and also given in the Table 3.3 . 


\subsection{Heat transfer (HT) in water}

The temperature increase in the water, $T_{\mathrm{w}}$, due to the laser-particle interaction and plasma heating by the laser pulse is modelled by solving the heat transfer equation. There were four types of heat sources: 1) interface conductance at the nanoparticle surface, given by equation (2.9); 2) Joule heating by the plasma interaction with the laser pulse in the vicinity of the nanoparticle, $Q_{\mathrm{rh}} ; 3$ ) electron collision losses with neutral molecules during impact ionization, $\left(\frac{d T_{\mathrm{w}}}{d t}\right)_{\text {coll }}$, defined in the Table 3.4, 4) heating through electron recombination of ionized molecules, $\left(\frac{d T_{\mathrm{w}}}{d t}\right)_{\text {rec }}$ (see Table 3.4 for details). Heat transfer in the water is modelled as,

$$
\rho_{\mathrm{w}} c_{\mathrm{w}} \frac{\partial T_{\mathrm{w}}}{\partial t}=\nabla\left(\kappa_{\mathrm{w}} \nabla T_{\mathrm{w}}\right)+Q_{\mathrm{rh}}+\left(\frac{d T_{\mathrm{w}}}{d t}\right)_{\text {coll }}+\left(\frac{d T_{\mathrm{w}}}{d t}\right)_{\mathrm{rec}} .
$$

Heating of the nanoparticle's lattice and the consequent heat diffusion to the surrounding water will lead to a temperature increase in the water possibly resulting in cavitation or bubble formation [122. The details on electron collision losses with neutral molecules during impact ionization and heating through electron recombination of ionized molecules are given in Table 3.4 .

\subsection{Plasma generation in water}

While cavitation and bubble formation around a gold nanoparticle can be achieved via photon absorption by the medium and the particle, ultrashort pulses provide an additional mechanism for bubble formation in the vicinity of the nanoparticle. This mechanism is LIOB which is defined as the nonlinear photon absorption by the media, which is sometimes also defined as the generation of free electron plasma. The processes of free electron plasma formation and LIOB in water without nanoparticles depicted in Figure 2.2. This process is based on multiphoton ionization (MPI) and avalanche ionization (AI). Sacchi, in 1991, purposed the treatment of water as an amorphous semiconductor with excitation energy of $6.5 \mathrm{eV}$ (energy required to excite electrons from $1 b_{1}$ orbital to the excitation band). This approach was adapted by others, where water was treated with a band gap energy of $6.5 \mathrm{eV}$.

The electrons occupying valence band can be excited to overcome the band gap by 


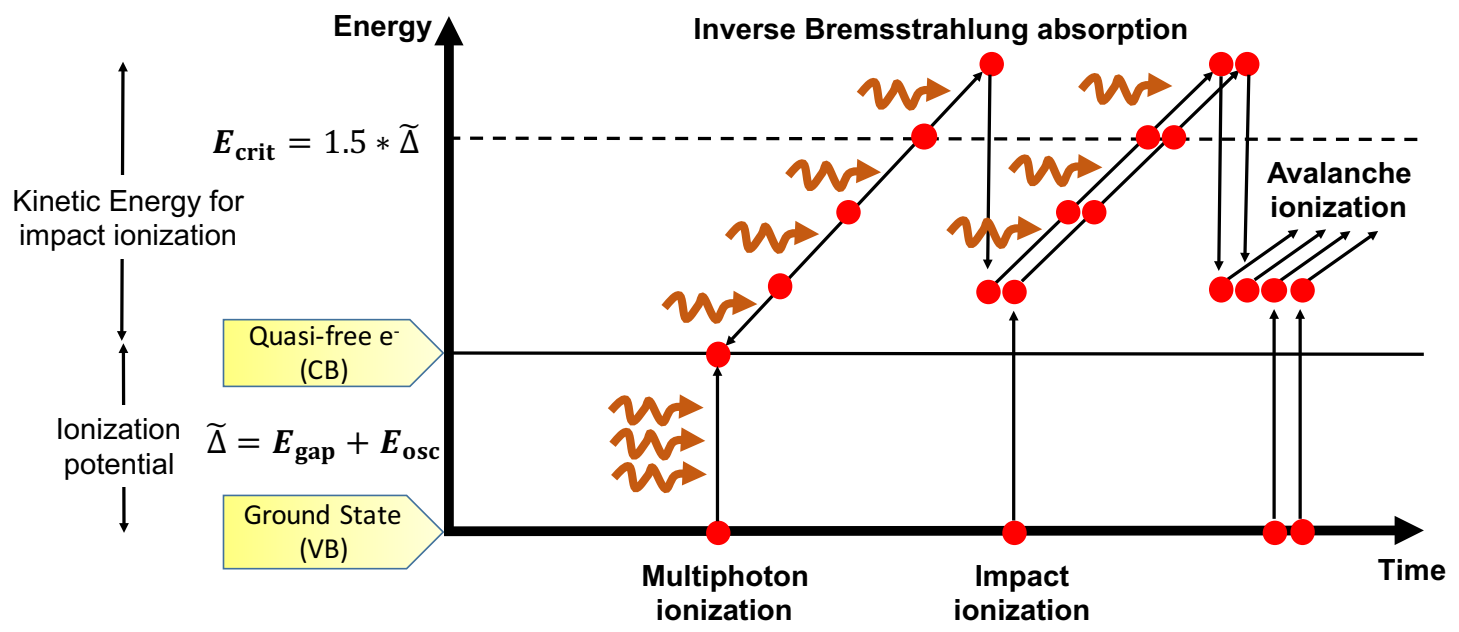

Figure 2.2: A schematic of the laser induced optical breakdown processes in water. During an ultrashort laser pulse interaction with water molecules, ground state electrons (in the VB) can overcome the bandgap, $\tilde{\Delta}$, by multiphoton ionization (MPI). Quasi-free electrons, excited electrons with sufficient kinetic energy to escape local potential energy barriers, continue to gain kinetic energy via absorption of the photons. This process is called inverse Bremsstrahlung absorption (IBA). Several IBA events by quasi-free electrons allows to gain a sufficient kinetic energy $\left(E_{\text {crit }}\right)$ so that quasi-free electron can ionize another electron in the ground state by molecular collision. This process is called impact ionization. Recurring sequences of IBA and impact ionization leads to an ionization cascade (IC), which also known as avalanche ionization (AI).

either MPI or tunneling [114, 123], or by impact ionization [77, 124]. It is assumed that quasi-free electron can be produced by the excitation from the valence band (VB) if the sum of the simultaneously absorbed photons (MPI) or the kinetic energy of an impacting free electron exceeds band gap energy, $E_{\text {gap }}$ (where $E_{\text {gap }}=6.5 \mathrm{eV}$ is assumed). An effective ionization potential, $\tilde{\Delta}$, can be assumed during LIOB with ultrashort pulses to account for the oscillation energy of the electron due to the electric field of laser pulse 
(see Figure 2.2). This ionization potential is given by

$$
\tilde{\Delta}=E_{\text {gap }}+\frac{e^{2} E^{2}}{4 m^{\prime} \omega^{2}}
$$

where $e$ is the electron charge, $E$ is the amplitude of the electric laser field, $\omega$ is the angular frequency of the light, and $m^{\prime}$ is the exciton reduced mass $\left(1 / m^{\prime}=1 / m_{\mathrm{c}}+1 / m_{\mathrm{v}}\right.$, where $m_{\mathrm{c}}$ is the effective mass of the quasi-free electron in the conduction band, and $m_{\mathrm{v}}$ is the effective mass of the hole in the valence band).

Once a quasi-free electron is produced by MPI, it can further absorb energy of a photon through an inverse Bremsstrahlung absorption (IBA) process. When the quasifree electron gains sufficient kinetic energy through an IBA, given by $E_{\text {crit }}=1.5 * \tilde{\Delta}$, it can ionize another electron in the ground state by molecular collision (impact ionization). The recurring sequences of inverse Bremsstrahlung absorption and impact ionization starts an avalanche ionization (AI), also known as ionization cascade (IC). Avalanche ionization can lead to critical free electron density that initiates bubble formation.

\subsubsection{The full plasma rate equation}

To model the changes of the free electron density and to determine the irradiance threshold for optical breakdown, the generic form of the rate equation for free electron density, $\rho_{\mathrm{e}}$, was used [27]:

$$
\frac{d \rho_{\mathrm{e}}}{d t}=\left(\frac{d \rho_{\mathrm{e}}}{d t}\right)_{\text {photo }}+\left(\frac{d \rho_{\mathrm{e}}}{d t}\right)_{\text {casc }}+\left(\frac{d \rho_{\mathrm{therm}}}{d t}\right)+\left(\frac{d \rho_{\mathrm{e}}}{d t}\right)_{\text {diff }}+\left(\frac{d \rho_{\mathrm{e}}}{d t}\right)_{\text {rec }} .
$$

The $\left(\frac{d \rho_{\mathrm{e}}}{d t}\right)_{\text {photo }}$ term models photoionization of electrons via multiphoton absorption and tunnel ionization [114, 125, 126]. The $\left(\frac{d \rho_{\mathrm{e}}}{d t}\right)_{\text {casc }}$ term adds the contribution of AI (sometimes called ionization cascade) via requiring sequences of IBA and impact ionization [127-129]. The $\left(\frac{d \rho_{\text {therm }}}{d t}\right)$ term adds the thermal ionization rate, that models the free electron thermal emission in the water [59]. The $\left(\frac{d \rho_{\mathrm{e}}}{d t}\right)_{\text {diff }}$ and $\left(\frac{d \rho_{\mathrm{e}}}{d t}\right)_{\mathrm{rec}}$ terms represent free electron plasma diffusion and recombination, respectively. The details for diffusion, $\left(\frac{d \rho_{\mathrm{c}}}{d t}\right)_{\text {diff }}$, and electron-hole recombination rates, $\left(\frac{d \rho_{\mathrm{e}}}{d t}\right)_{\text {rec }}$ of equation 2.13 are given in the Table 3.4. The former is based on the characteristic diffusion length, $\Lambda$, which is set to the effective radius of the gold nanoparticle [82], while the latter is set to an empirical 
value obtained by Docchio [130].

For a complete picture of the free electron generation in the vicinity of a gold nanoparticle in water a boundary condition on the gold's surface is needed to introduce a photothermal emitted current density as a source of free electrons.

\subsubsection{Photoionization}

The photoionization of electrons is governed by the mechanisms of MPI and tunneling where the probability of each is determined by the electric field strength, $E$, and the frequency of the electromagnetic field, $\omega$. In order to distinguish between the two regimes, Keldysh [114] introduced a parameter, $\gamma$, which is given by

$$
\gamma=\frac{\omega}{\omega_{\mathrm{t}}}=\omega \frac{\sqrt{m^{\prime} E_{\mathrm{gap}}}}{e|\mathbf{E}|}=\frac{\omega}{e} \sqrt{\frac{c_{0} \varepsilon_{0} n m^{\prime} E_{\mathrm{gap}}}{2 I_{\mathrm{tot}}(t)}}
$$

where $1 / \omega_{\mathrm{t}}$, is the tunnelling time through the atomic potential barrier, $c_{0}$ and $\varepsilon_{0}$ are the electromagnetic constants for vacuum, $n$ is the refractive index of medium, $|\mathbf{E}|$ is the magnitude of the electric field, and $I_{\text {tot }}$ is the total irradiance of the laser pulse. It should be noted, that due to presence of the nanoparticle and LSPR that enhance the electric field in the particle's vicinity, the Keldysh parameter should be calculated based on the spatially varying electric field, rather then total irradiance. Furthermore, if the free electron density in the vicinity of a gold nanoparticle exceeds $10^{18} \mathrm{~cm}^{-3}$, the surroundings becomes a conductive medium, and it starts to absorb the incoming laser radiation and shields the nanoparticle. This affects the LSPR, the electric field enhancement and $\gamma$. For low frequencies and large field strengths, $\gamma<<1$ and therefore tunneling is responsible for the ionization. In the optical frequency range and at moderate electric field strengths, $\gamma>>1$, and the probability of MPI is much higher than tunneling.

Using the Keldysh parameter the effective ionization potential (equation 2.12) for creating an electron-hole pair in a condensed matter exhibiting a band structure and corrected for the oscillation energy of the electron due to the electromagnetic field, can be written as

$$
\tilde{\Delta}=E_{\text {gap }}\left(1+\frac{1}{4 \gamma^{2}}\right)=\frac{2}{\pi} E_{\text {gap }} \frac{\sqrt{1+\gamma^{2}}}{\gamma} \mathcal{E}\left(\frac{1}{\sqrt{1+\gamma^{2}}}\right)
$$


where $\mathcal{E}()$ denote the elliptic integrals of the second kind.

At room temperature the electron density in the ground state (VB) is $\rho_{\text {bound }}=6.68 \times 10^{22} \mathrm{~cm}^{-3}$ [58. During photoionization, $\rho_{\text {bound }}$ is reduced by $\rho_{\mathrm{e}}$. On the other hand, free electrons can come from PTE off gold nanoparticle surface, $\rho_{\text {au }}$, which are accounted in the total free electron density $\rho_{\mathrm{e}}$. Taking these into consideration, the total photoionization rate will be scaled by the dimensionless depletion factor $\left(\frac{\rho_{\text {bound }}-\left(\rho_{\mathrm{e}}-\rho_{\mathrm{au}}\right)}{\rho_{\mathrm{bound}}}\right)$.

The photoionization rate, $\left(\frac{d \rho_{\mathrm{e}}}{d t}\right)_{\text {photo }}$, is then given by [59]:

$$
\begin{array}{r}
\left(\frac{d \rho_{\mathrm{e}}}{d t}\right)_{\text {photo }}=\frac{2 \omega}{9 \pi}\left(\frac{m^{\prime} \omega \sqrt{1+\gamma^{2}}}{\hbar \gamma}\right)^{3 / 2} \mathrm{Q}\left(\gamma, \frac{\tilde{\Delta}}{\hbar \omega}\right) \times\left(\frac{\rho_{\text {bound }}-\left(\rho_{\mathrm{e}}-\rho_{\mathrm{au}}\right)}{\rho_{\text {bound }}}\right) \\
\exp \left\{-\pi\left\langle-\frac{\tilde{\Delta}}{\hbar \omega}+1\right\rangle \times\left[\mathcal{K}\left(\frac{\gamma}{\sqrt{1+\gamma^{2}}}\right)-\mathcal{E}\left(\frac{\gamma}{\sqrt{1+\gamma^{2}}}\right)\right] / \mathcal{E}\left(\frac{1}{\sqrt{1+\gamma^{2}}}\right)\right\}, \\
\mathrm{Q}(\gamma, \mathrm{x})=\sqrt{\frac{\pi \mathcal{K}\left(\frac{1}{\sqrt{1+\gamma^{2}}}\right)}{\text { where }}} \times \sum_{l=0}^{\infty} \exp \left\{-\pi l\left[\mathcal{K}\left(\frac{\gamma}{\sqrt{1+\gamma^{2}}}\right)-\mathcal{E}\left(\frac{\gamma}{\sqrt{1+\gamma^{2}}}\right)\right] / \mathcal{E}\left(\frac{1}{\sqrt{1+\gamma^{2}}}\right)\right\} \\
\times \Phi\left\{\left[\pi^{2}(2\langle x+1\rangle-2 x+l) / 2 \mathcal{K}\left(\frac{1}{\sqrt{1+\gamma^{2}}}\right) \mathcal{E}\left(\frac{1}{\sqrt{1+\gamma^{2}}}\right)\right]^{1 / 2}\right\} .
\end{array}
$$

$\langle x>$ represents the integer part of the number $x, \mathcal{K}()$ and $\mathcal{E}()$ denote the elliptic integrals of the first and second kind, and $\Phi()$ denotes the Dawson probability integral; given by

$$
\Phi(z)=\int_{0}^{z} \exp \left(y^{2}-z^{2}\right) d y
$$

\subsubsection{Avalanche ionization (AI)}

After a free electron produced in the field of a laser light, it can gain kinetic energy through IBA. Once the kinetic energy of the free electron exceeds the critical energy, $E_{\text {crit }}=\left(\frac{1+2 \mu}{1+\mu} \tilde{\Delta}\right)$, where $\mu$ depends on the band structure and assumed to be equal to 1 [59, it can generate another free electron through an impact ionization. The resulting 
rate of avalanche ionization rate per electron is then given by [58]:

$$
\eta_{\text {casc }}=\frac{1}{\omega^{2} \tau^{2}+1}\left[\frac{\mathrm{e}^{2} \tau}{c_{0} n \varepsilon_{0} m_{\mathrm{e}}(3 / 2) \tilde{\Delta}} I_{\mathrm{tot}}(t)-\frac{m_{\mathrm{e}} \omega^{2} \tau}{M}\right]
$$

where $\tau$ is the mean time between collisions and $M$ is the mass of water molecule (see Table 3.4).

AI only starts after a certain free electron density, $\rho_{\text {seed }}$, has been reached, either by photoionization or PTE. In addition, there is a finite time between electron/molecule collisions during IBA. Therefore, AI starts at a retarded time, $t_{\mathrm{ret}}$, to account for the mean free time between collisions and the time it takes for the photons to be absorbed in order to gain sufficient kinetic energy. $\rho_{\text {seed }}$ is a fitting parameter that is chosen based on $50 \%$ probability of having at least one seed electron in the focal volume of the laser [59].

The avalanche ionization rate, $\left(\frac{d \rho_{\mathrm{e}}}{d t}\right)_{\text {casc }}$, can be written as [59]:

$$
\left(\frac{d \rho_{\mathrm{e}}}{d t}\right)_{\text {casc }}= \begin{cases}\frac{\alpha_{\text {casc }} I_{\text {tot }}(t)}{1+\eta_{\text {casc }} t_{\text {ret }}} \rho_{\mathrm{e}}-\frac{\beta_{\text {casc }}}{1+\eta_{\text {casc }} t_{\text {ret }}}\left(\rho_{\mathrm{e}}-\rho_{\text {therm }}\right) & \text { for } \rho_{\mathrm{e}} \geq \rho_{\text {seed }} \\ 0 & \text { for } \rho_{\mathrm{e}}<\rho_{\text {seed }}\end{cases}
$$

where $\alpha_{\text {casc }}$ is the gain in ionization cascade, $\beta_{\text {casc }}$ is the collision loss term of ionization cascade and $\rho_{\text {therm }}$ is the free electron density generated through thermal ionization (TI).

\subsubsection{Thermal ionization (TI) and Photo-thermal emission (PTE)}

To complete the picture of a free electron plasma formation during a single picosecond or nanosecond pulse or femtosecond pulse sequences and in the presence of a gold nanoparticle, two additional processes need to be addressed: thermal ionization (TI) [59, 66], $\rho_{\text {therm }}$, and photo-thermal emission (PTE) of electrons off nanoparticle surface, $\rho_{\mathrm{au}}$, respectively 62 .

TI starts to provide a significant source of free electrons when the temperature of water in the focal volume of the laser is sufficiently high $(\geq 5000 \mathrm{~K}[59$, 66]) or when large free electron densities $\left(\rho_{\mathrm{e}}>10^{21} \mathrm{~cm}^{-3}\right)$ are already reached through MPI and AI. Linz et al. [59] derived a thermal ionization rate equation 2.20, $\left(\frac{d \rho_{\text {therm }}}{d t}\right)$, which is based on the process of thermal emission of free electrons during the thermalization of an energy 
carried by primary free electrons via collisions and recombination.

Since the thermalization time of free electrons is long (on the order of several picoseconds), during femtosecond pulses in water the thermal emission will only occur after the laser pulse. While during nanosecond pulses, which are 2 orders of magnitude longer than the thermalization time, a rapid heating of the laser focal volume during laser pulse will provide a significant number of electrons to support AI through TI [59, 66]. TI also partially depletes the density of bound electrons in the valence band and reduces the rate of multiphoton and impact ionization [59]. This picture is changing during nanoparticlemediated optical breakdown in water, where free electrons that are generated by MPI, AI and PTE can rapidly reach high densities $\left(\rho_{\mathrm{e}}>10^{21} \mathrm{~cm}^{-3}\right)$ during fs pulses. The recombination and collisions of these primary free electrons through energy thermalization will rapidly increase the water's temperature so that TI will play a significant role in the free electron generation. Therefore, TI should also be accounted for femtosecond and picosecond pulses during nanoparticle-mediated optical breakdown. Under the conditions studied in this thesis, the temperature of the water during the single pulse illumination was lower than $5000 \mathrm{~K}$, TI was still included in equation (2.13), in order to have a complete model of the free electron plasma generation for further use.

Thermal ionization rate, $\left(\frac{\partial \rho_{\text {therm }}}{\partial t}\right)$, of free electron into conduction band is given by [59]:

$$
\left(\frac{\partial \rho_{\text {therm }}}{\partial t}\right)=\frac{\partial T_{\mathrm{w}}}{\partial t}\left(\frac{3 \kappa_{\mathrm{b}}}{E_{\text {gap }}}+\frac{1}{T_{\mathrm{w}}}\right) \frac{3}{2} \sqrt{\frac{\pi}{2}}\left(\frac{\kappa_{\mathrm{b}} T_{\mathrm{w}}}{E_{\text {gap }}}\right)^{\frac{1}{2}} \exp \left(-\frac{E_{\text {gap }}}{2 \kappa_{\mathrm{b}} T_{\mathrm{w}}}\right)\left(\rho_{\text {bound }}-\rho_{\mathrm{e}}\right) .
$$

PTE of hot electrons on the boundary of the gold, also starts to play a role when the temperature of the nanoparticle's electrons rises to a level where the electrons can cross the metal/medium energy barrier of $W_{\mathrm{au}}=3.72 \mathrm{eV}$ and contribute to free electron density plasma formation [131].

Photo-thermally emitted current density, $\boldsymbol{J}_{\mathrm{au}}$, across metal/medium boundary can be described by the generalized Fowler-DuBridge theory of multiphoton photoemission at high temperatures [61, 132]:

$$
\boldsymbol{J}_{\mathrm{au}}=\left[A_{0} T_{\mathrm{e}}^{2} \exp \left(-\frac{W_{\mathrm{au}}}{\kappa_{\mathrm{b}} T_{\mathrm{e}}}\right)+c_{\mathrm{au}} \frac{2\left(\kappa_{\mathrm{b}} T_{\mathrm{e}}\right)^{2}}{\left(3 \hbar \omega-W_{\mathrm{au}}\right)^{2}} F\left(\frac{\left(3 \hbar \omega-W_{\mathrm{au}}\right)}{\kappa_{\mathrm{b}} T_{\mathrm{e}}}\right)(1-R)^{3} I_{\mathrm{tot}}^{3}(t)\right] \mathbf{n},
$$


where $A_{0}$ is the Richardson coefficient $\left(120 \mathrm{~A} / \mathrm{cm}^{2} / \mathrm{K}^{2}\right)$ [131, $F$ is the Fowler function [61, 132, $c_{\text {au }}$ is the three-photon ionization cross-section $\left(1 \times 10^{-7} \mathrm{~A} \cdot \mathrm{cm}^{4} / \mathrm{MW}^{3}\right)$ [133] and $R$ is the reflection coefficient of the gold. The photo-thermal electron current density is set as a boundary flux/source condition across gold/water interface.

\subsubsection{Nanoparticle-mediated optical breakdown}

The presence of gold nanoparticles in water will lower the optical breakdown threshold in comparison to the pure water alone. Boulais et al. [67] theoretically determined that there are two primary regimes of a nanoparticle-mediated optical breakdown: the absorption regime and the near-field regime. Both of the regimes are depicted in Figure 2.3. When a gold nanoparticle interacts with a laser pulse it generates a plasmon resonance. This results in electric field enhancement in the vicinity of the particle. Depending on the laser fluence, the type of gold nanoparticle and the surrounding medium it can either linearly absorb photons (the absorption regime) or it can exhibit both linear absorption by the particle and non-linear absorption by the medium (the near-field regime). The absorption regime occurs at a lower fluence than the near-field regime. The absorption regime will

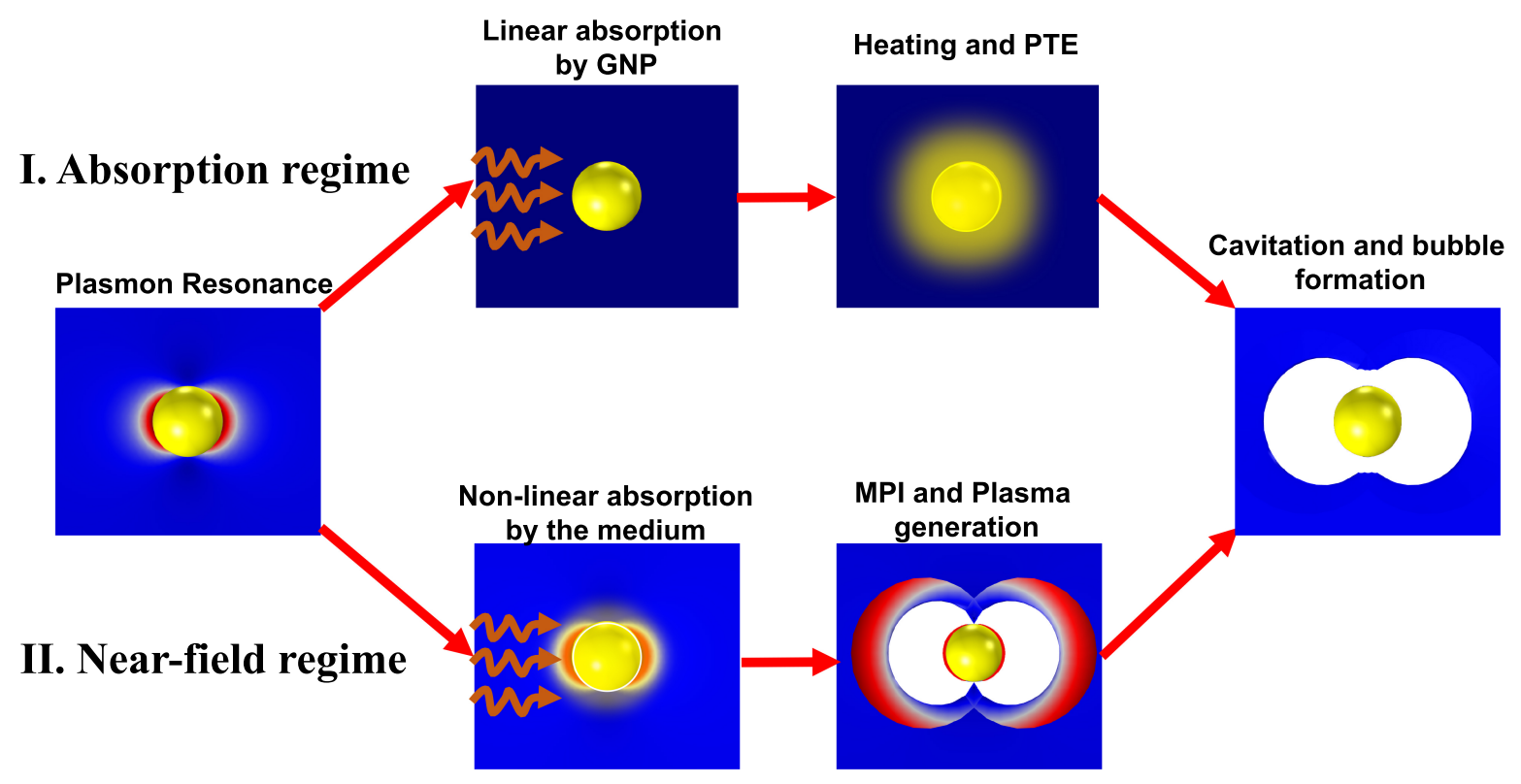

Figure 2.3: Schematic of two regimes of nanoparticle-mediated optical breakdown. 
lead to heating of the nanoparticle, producing PTE of electrons off the nanoparticle surface that are the seed electrons for AI. While in the near-field regime the rate of MPI in the medium will generate a high density plasma in the vicinity of the particle, so that nanoparticle becomes shielded from the incoming laser radiation. Shielding of the nanoparticle will reduce the nanoparticle heating and the PTE rate. In both regimes, if the seed electron density is high enough to trigger avalanche ionization, the optical breakdown will occur, leading to cavitation and bubble formation.

\subsubsection{The wavelength dependence of optical breakdown in water}

Although $6.5 \mathrm{eV}$ is often used as a band gap energy of water in computational modelling of optical breakdown [62, 67, 82, 83, 97, 99], the actual band gap structure of water is more complex. Recent spectroscopic experimental findings has suggested that the approach proposed by Sacchi [53] of separating the VB and the CB by an energy gap of $6.5 \mathrm{eV}$ is too simplistic. The ionization and geminate recombination pathways in water are schematically shown in Figure 2.4. In reality, the effective direct ionization into the $\mathrm{CB}$ happens at excitation energies higher than $9.5 \mathrm{eV}[78,79]$. A number of $6.5 \mathrm{eV}$ that was used as the ionization energy is actually the minimum energy needed for promotion of VB electrons into the solvated state (the $\tilde{A} 1^{1} B_{1}$ absorption band, which reaches down to $6 \mathrm{eV})$, so called "initiation energy", $E_{\mathrm{ini}}$. A solvated electron, $e_{\mathrm{aq}}^{-}$, that escapes geminate recombination with its hydronium counterion within 200 ps [134] is then upconverted into the CB due to smaller energy gap $(3 \mathrm{eV})$ and a large absorption cross-section of $e_{\text {aq }}^{-}$ and their excited $p$ states.

Linz et al. [59] studied the wavelength dependence of the optical breakdown threshold in water for ns and fs pulses. They hypothesized that when the optical breakdown initiation in water depended on MPI then the wavelength dependence of the optical breakdown threshold should exhibit a sharp rise in the wavelength region where one more photon is required to ionize electron to CB [74]. This is illustrated in Figure 2.5, where the MPI rate exhibits step decreases in the regions where multiphoton order jumps from 4 photons per ionization to 5 photons per ionization, at around $750 \mathrm{~nm}$ and from 5 photons per ionization to 6 photons per ionization at around $940 \mathrm{~nm}$. On the other hand, the AI rate is also has a wavelength dependence and increases with the wavelength (Figure 2.5). The monotonic increase of the AI rate with the wavelength makes it possible 


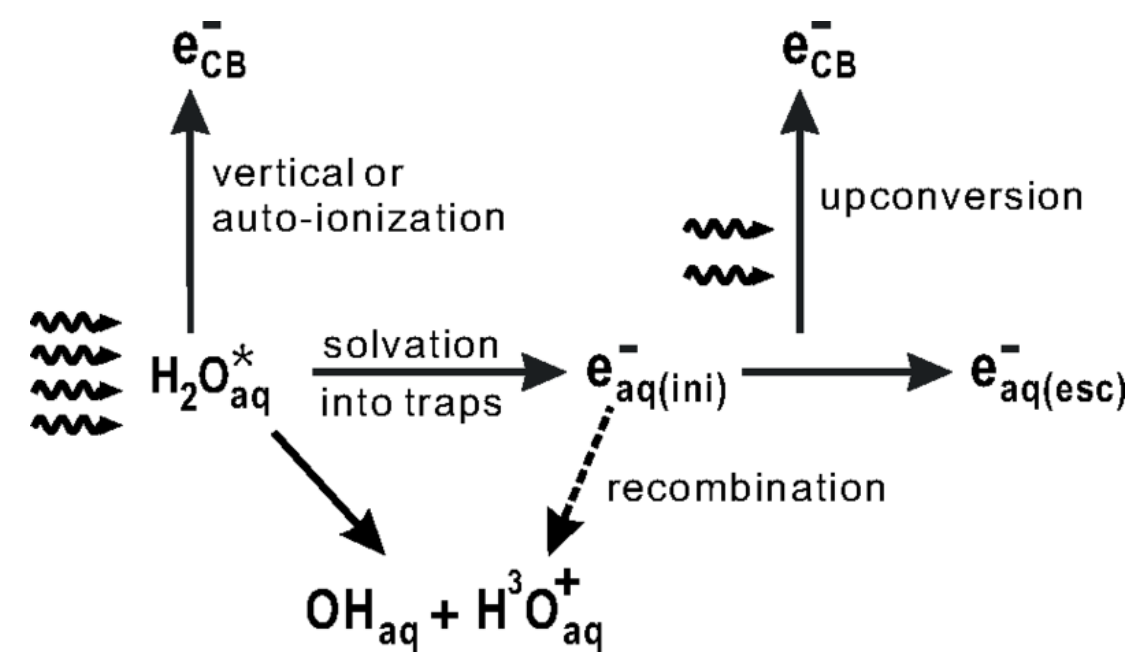

Figure 2.4: Ionization and geminate recombination pathways in liquid water. For large excitation energies, $E_{\text {exc }}$, ionization can proceed via vertical ionization $\left(E_{\text {exc }} \geqslant\right.$ $11 \mathrm{eV})$ or autoionization $\left(9.5 \mathrm{eV} \leq E_{\text {exc }}<11 \mathrm{eV}\right)$, while for $E_{\text {exc }}<9.5 \mathrm{eV}$, ionization is possible only as a two-step process involving solvated electron creation followed by upconversion of $e_{\mathrm{aq}}^{-}$into the $\mathrm{CB}$. The latter process competes with geminate recombination. Reprinted with permission from [74].

to initiate optical breakdown at a longer wavelengths with a lower $\rho_{\text {seed }}$.

The hypothesis was confirmed experimentally for nanosecond pulses in water, as shown in Figure 2.6 [74]. The optical breakdown irradiance threshold, $I_{\mathrm{th}}(\lambda)$, exhibit stepwise behaviour at the transition zone of MPI process order $\kappa$ to $(\kappa+2)$.

Linz et al. [74] compared the experimental results against predictions from a simplified optical breakdown model (Figure 2.7). The use of a constant $\rho_{\text {seed }}$, as was described in Section 2.4.3 and equation 2.19), for AI initiation and the optical breakdown threshold did not agree well with experimental data (see Figure 2.7 ). On the other hand, due to the role of MPI in breakdown initiation and $\rho_{\text {seed }}$ production, the stepwise behaviour of optical breakdown threshold predicted.

A better agreement against the experimental results was reached when $\rho_{\text {seed }}$ was modelled as a wavelength dependent parameter (Figure 2.7 a). The agreement was improved 


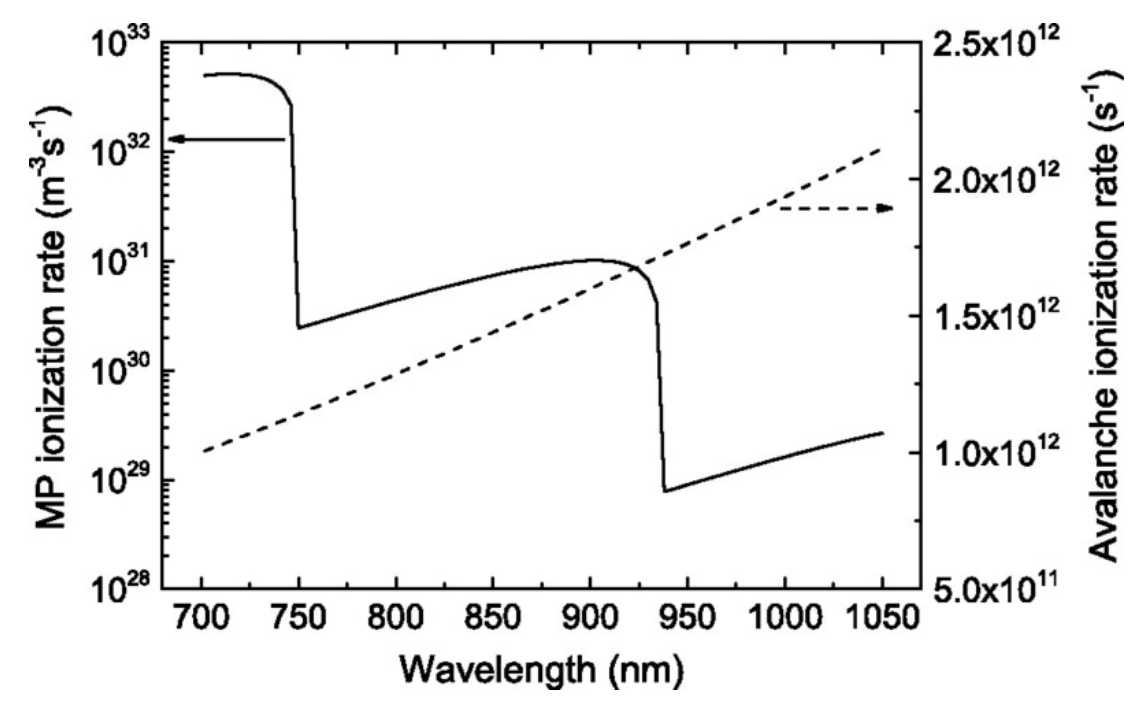

Figure 2.5: Wavelength dependence of AI rate (dashed line) and MPI rate (solid line) calculated for $E_{\text {gap }}=9.5 \mathrm{eV}$ and $E_{\text {ini }}=6.6 \mathrm{eV}$, respectively. The MPI rate is plotted in log scale, while AI rate is in linear scale. All calculations assume an irradiance of $3.5 \times 10^{11} \mathrm{~W} / \mathrm{cm}^{2}$, which is the average optical breakdown threshold value in the investigated wavelength range. Reprinted with permission from [74].

when both $\rho_{\text {seed }}(\lambda)$ and $E_{\text {ini }}(\lambda)$ were modelled having a wavelength dependence (Figure $2.7 \mathrm{~b}$ ). In order to properly model the water ionization, one should use $E_{\text {gap }}=9.5 \mathrm{eV}$ (which will adequately account for both, vertical $(11 \mathrm{eV})$ and autoionization $(9.5 \mathrm{eV})$ processes in water $)$ and add a separate "initiation channel" with $E_{\text {ini }}=-(0.0012 \mathrm{eV} / \mathrm{nm}) \lambda+7.58 \mathrm{eV}$ (for ionization through the intermediate solvated state), and use $\rho_{\text {seed }}=\left(1 \mathrm{~cm}^{-3}\right) 10^{-(0.01116 / \mathrm{nm}) \lambda+23.5}$ [74. The wavelength dependence of both $\rho_{\text {seed }}$ and $E_{\text {ini }}$, is associated with a rate of avalanche ionization that increases with wavelength [74].

The existence of a separate excitation channel for optical breakdown initiation, through the creation of solvated electrons, and a wavelength dependence of the seed electron density is often ignored in models of nanoparticle-mediated optical breakdown in water (see Table 1.1. 


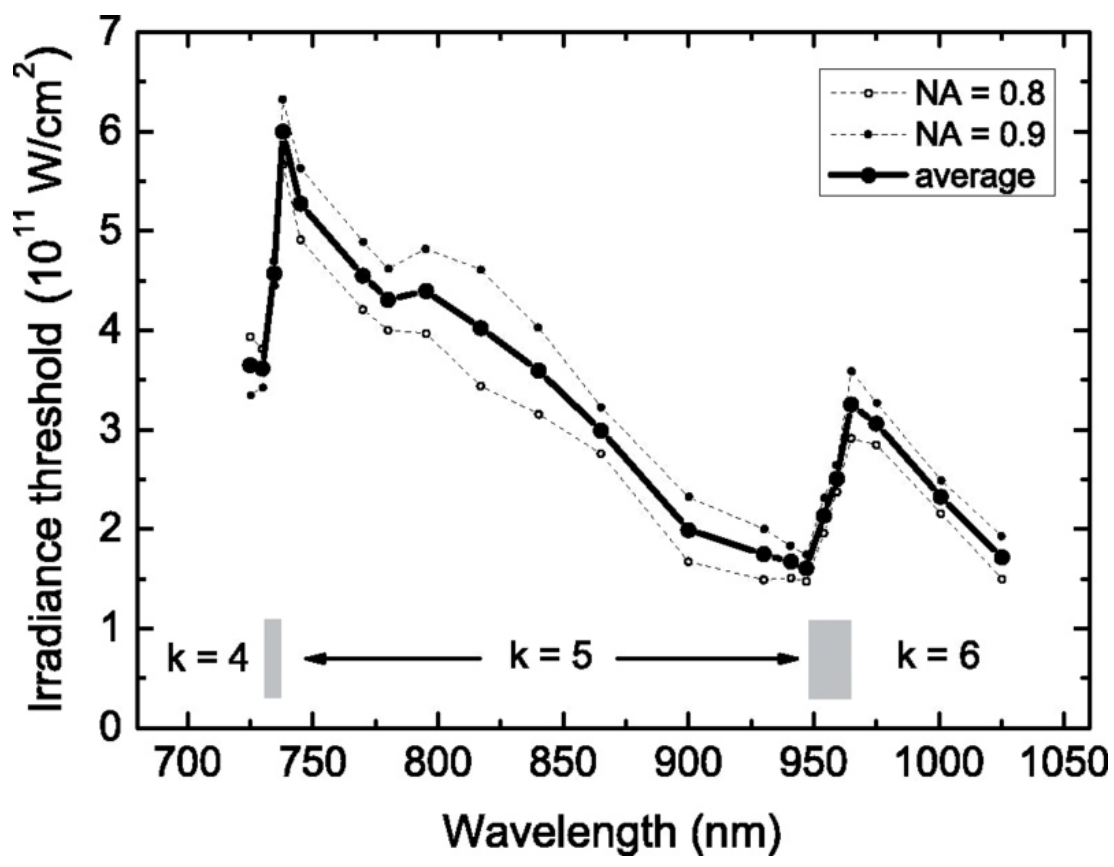

Figure 2.6: Wavelength dependence of the threshold, $I_{\mathrm{th}}(\lambda)$, for plasma-mediated bubble formation by SLM OPO pulses focused at $\mathrm{NA}=0.8$ and $\mathrm{NA}=0.9$ and averaged values. The order of the multiphoton process required to cross the band gap in different regions of the $I_{\mathrm{th}}(\lambda)$ spectrum is denoted by $\kappa$. Transition zones are marked in grey. Reprinted with permission from [74]. 


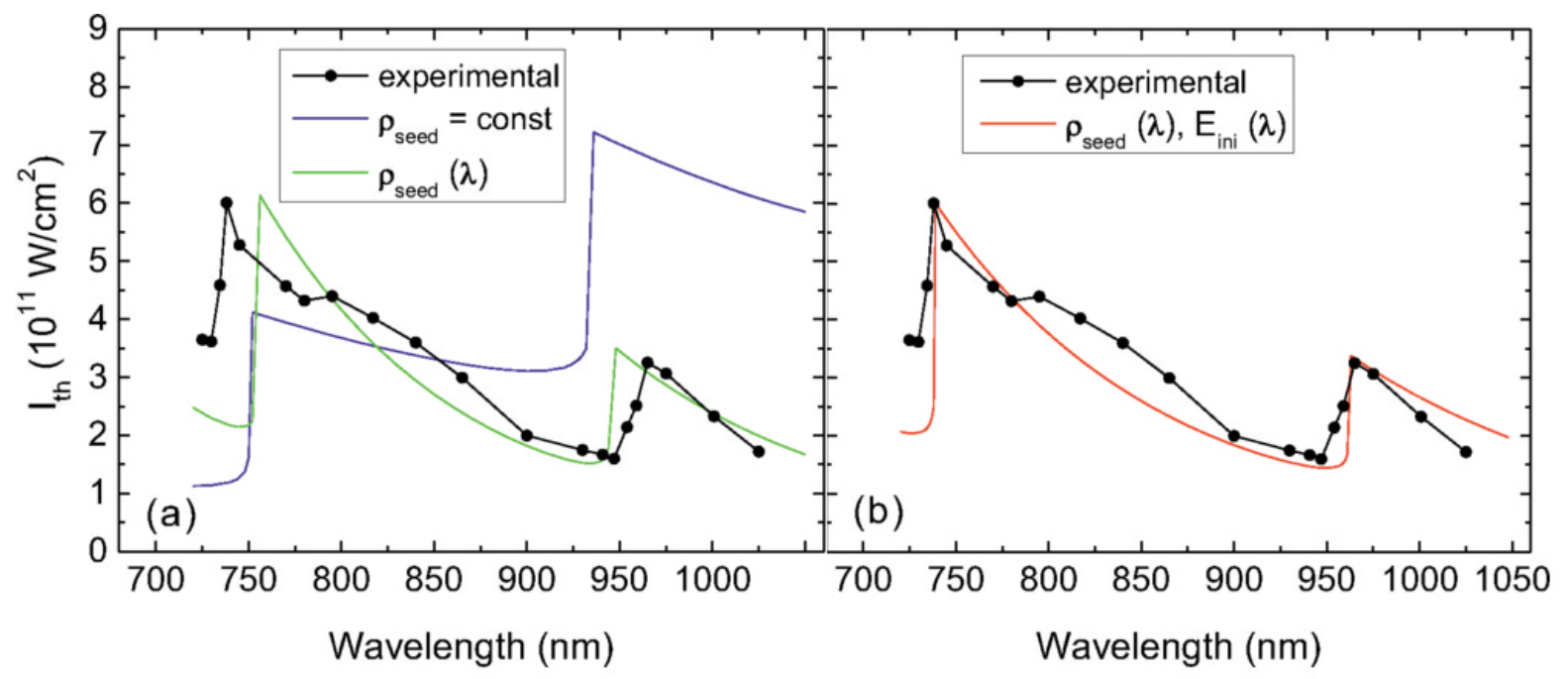

Figure 2.7: Comparison of predictions for $I_{\mathrm{th}}(\lambda)$ based on simplified optical breakdown model, with the experimentally determined spectrum (average values from both NAs). Calculations were performed for $2 \mathrm{~ns}$ pulse duration. (a) Fits for $E_{\text {ini }}=6.6 \mathrm{eV}$ assuming either a constant $\rho_{\text {seed }}=3.3 \times 10^{14} \mathrm{~cm}^{-3}$ (blue line) or a wavelength dependent seed electron density that varies according to $\rho_{\text {seed }}\left(\mathrm{cm}^{-3}\right)=10^{A \lambda(n m)+B}$ (green line). (b) Fit assuming a wavelength dependent $\rho_{\text {seed }}$ and a linear decrease of $E_{\text {ini }}$ from $6.7 \mathrm{eV}$ at the position of the first peak to $6.43 \mathrm{eV}$ at the second peak (red line; for fit parameters see text). Reprinted with permission from [74]. 


\section{Chapter 3}

\section{Methods}

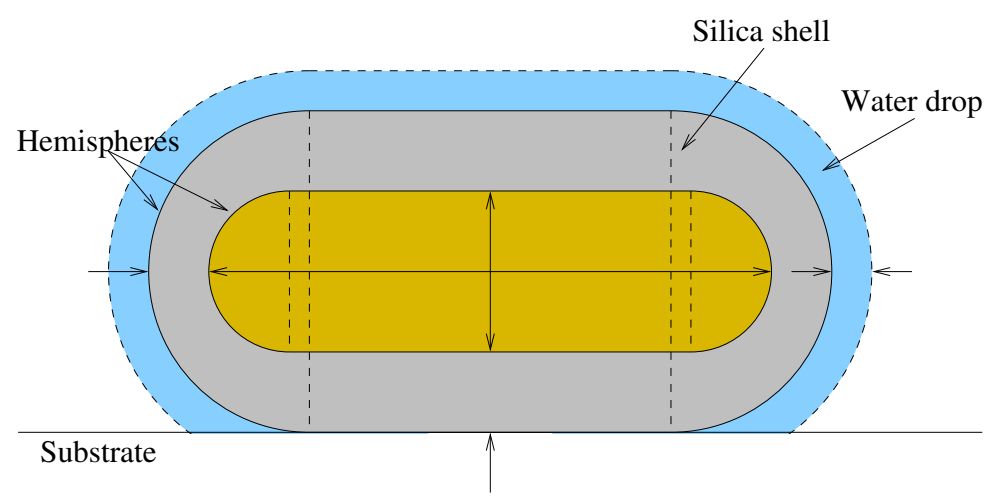

The developed theoretical model of nanoparticle-mediated LIOB was used to study the wavelength, environment and nanoparticle morphology dependence of the optical breakdown threshold, $I_{\mathrm{th}}$. This was done using the theory described in Chapter 2. Three investigations were performed using the model. First, the investigation of the sensitivity of bare versus silica-coated gold nanorods (GNR versus S-GNR) to their local environment was done to predict the effects that the range of hypothetical environmental conditions will have on the SPR of bare and silica-coated GNRs. In another study, the theoretical investigation of the role of gold nanoparticle morphology on the optical breakdown threshold was done. In the third study, the model was used to investigate the wavelength dependence of a nanoparticle-mediated optical breakdown in water during infrared femtosecond and picosecond pulse exposure of a gold nanorod. And finally, the model of nanoparticle-mediated optical breakdown was validated against published data.

\subsection{A finite element method based model}

The gold nanoparticle-mediated optical breakdown model was simulated using the finite element (FE) method, using the commercial software COMSOL Multiphysics. COMSOL Multiphysics is able to solve coupled partial differential equations and ordinary differential equations for arbitrary geometries in both time and frequency domains. The FE model included individual gold nanoparticles and coupled several physical problems together and simultaneously solved the partial differential equations for electromagnetic (EM) 
wave propagation (Section 2.1), a two-temperature model (TTM) of heat transfer in gold (Section 2.2), a model of heat transfer (HT) in water (Section 2.3), and plasma generation in water (Section 2.4). The coupling of the nanoparticle-mediated optical breakdown process and the coupling variables are depicted in Figure 3.1. Several (properties of the medium and the gold nanoparticles) being temperature and wavelength dependent.

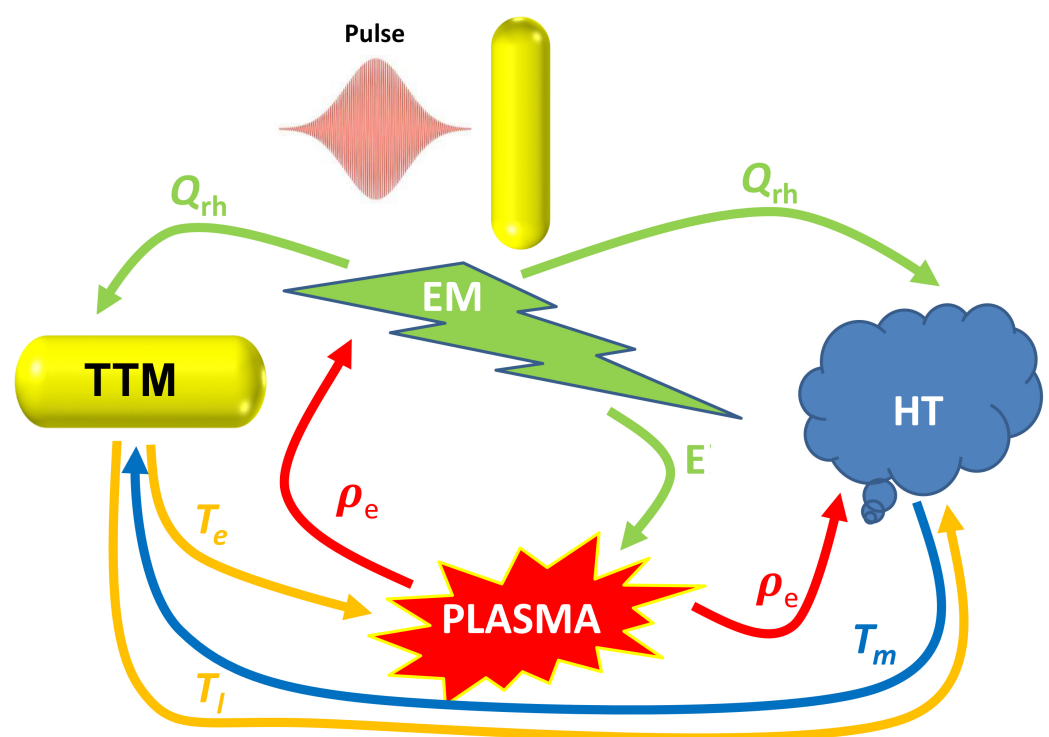

Figure 3.1: A schematic of the LIOB model coupling.

The full 3D geometry was reduced to one-quarter in the EM model by using perfect electric conductor (PEC) and perfect magnetic conductor (PMC) boundaries. All other physics domains, for example: TTM, heat transfer (HT), plasma model, were truncated to one-quarter using symmetry planes.

The EM wave propagation domain was reduced to eliminate reflections from an artificial boundaries, with a combination of a perfectly matched layer (PML) and an absorbing boundary condition (ABC). All domains were meshed using tetrahedral elements with quadrilateral vector basis function. The maximum mesh element size was kept below $\lambda / 10$, where $\lambda$ is the wavelength of light in the computational medium, with at least ten times smaller elements in the plasma and TTM domains. An iterative geometric multigrid solver was used to solve for electric field. The direct PARDISO solver with the nested dissection multithreaded algorithm [117] was used for all other models. The 
coupled model was solved in a combined frequency and time domain, using a backward differentiating with a maximum order of 2 [117].

The study of the influence of the environment on the optical behaviour of gold nanorods was done by individually measuring the extinction cross-section spectra, $\sigma_{\text {ext }}(\lambda)$, of three bare and three silica-coated GNRs using spatial modulation spectroscopy (SMS) [88, 103, 104. Compared to other single nanoparticle spectroscopy techniques SMS has the key advantage of providing the absolute spectrum instead of the relative one that other methods provide, which permits a comparison of experimental and theoretical results. Furthermore, as it is sensitive to both absorption and scattering, smaller particles can be investigated compared to commonly used scattering based techniques; scattering becoming negligible compared to absorption at small sizes. As s result the size detection limit for gold nanospheres is about $5 \mathrm{~nm}$ for SMS compared to $20 \mathrm{~nm}$ for scatter based spectroscopy (usually dark-field spectroscopy). In order to quantitatively compare the spectra of simulations against SMS measurements, the particles were placed on a TEM grid and the dimensions of each nanorod were obtained using TEM. Since SMS was performed with the particles on the TEM grid the model assume that the GNR was on a silica substrate in air.

\subsection{The role of the environment on the optical proper- ties of gold nanorods}

Simulations were than performed by solving the Maxwell's equations with the proper boundary conditions using FEM that included the TEM measured nanoparticle size and shape and a size-corrected dielectric function for gold. Using this a detailed theoretical quantitative analysis was performed on the role of environment on several spectral features. In order to do this, bare and silica-coated GNRs were first chemically synthesized as colloidal solutions in an aqueous solvent and spin coated onto a TEM grid.

\subsubsection{GNR Synthesis}

GNRs were synthesized by a seed-mediated growth method in the presence of the cetyltrimethylammonium bromide CTAB surfactant [135]. These nanorods are referred to here 
as bare GNRs. Silica-coated gold nanorods (S-GNR) were prepared by functionalization of cetyltrimethylammonium bromide (CTAB)-capped gold nanorods with a thiolmodified poly(ethylene glycol) prior to TEOS condensation [136]. The mean rod aspect ratio, $a=l / w$, was around 3.5 , with a mean width, $w$, of about $10 \mathrm{~nm}$ and a silica shell thickness, $s$, ranging from approximately 8 to $16 \mathrm{~nm}$ for the coated rods. After proper dilution, a drop of the solution was spin coated onto a $50 \mu \mathrm{m} \times 50 \mu \mathrm{m}$ window TEM grid covered by a $40 \mathrm{~nm}$ thick silica film. The latter provides the required substrate for depositing isolated nanoparticles together while permitting both optical and electron transmission measurements. Surface deposited individual GNRs separated by more than $1 \mu \mathrm{m}$ were obtained, permitting easy optical separation (Figure $3.2 \mathrm{~b}$ ).

\subsubsection{Optical Characterization}

Spatial modulation spectroscopy (SMS) [88, 108 was used to measure the extinction cross-section of individual GNRs (Figure 3.3). It is based on the modulation of a particle's spatial position at frequency $f$, in the focal spot of a tightly focused laser beam. The presence of a nanoparticle induces a modulation of the transmitted light power, with an amplitude proportional to its extinction cross-section $\sigma_{\text {ext }}(\lambda)$. The value of the extinction cross section $\sigma_{\text {ext }}(\lambda)$ of a single nanoparticle can thus be measured for sizes down to $5 \mathrm{~nm}$ in the case of gold spheres [88]. The $\sigma_{\text {ext }}(\lambda)$ spectrum was measured by tuning the wavelength of the light source. For the investigated rods, their longitudinal SPR, that is, for light polarized along their long axis, was observed at $800 \mathrm{~nm}$. Spectra were thus measured using a Ti:sapphire laser tunable in the 680 to $1080 \mathrm{~nm}$ spectral range. The laser beam was focused close to the diffraction limit using a $100 \times$ microscope objective with a numerical aperture of 0.75 , yielding a focal spot size of about $0.7 \lambda$ (full-width at half-maximum of the light intensity profile). The incident mean power was about $10 \mu \mathrm{W}$. The sample position was modulated at $f=1.5 \mathrm{kHz}$. The transmitted light power was collected after the sample by a second microscope objective identical to the focusing one, and detected by a photodiode. Demodulation of the transmitted light amplitude was performed at $2 f$ (by a lock-in amplifier) as it permits more precise localization of the particle [88. The spatial dependence of the signal is approximately proportional to the second derivative in the modulation direction of the beam intensity profile at the focal spot and directly proportional to the beam profile in the direction perpendicular 


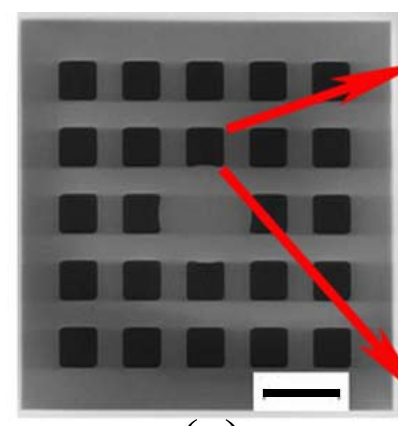

(a)

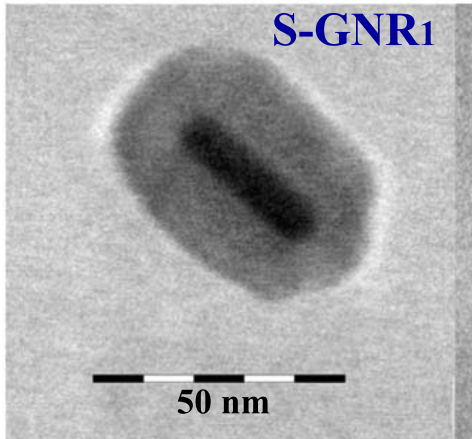

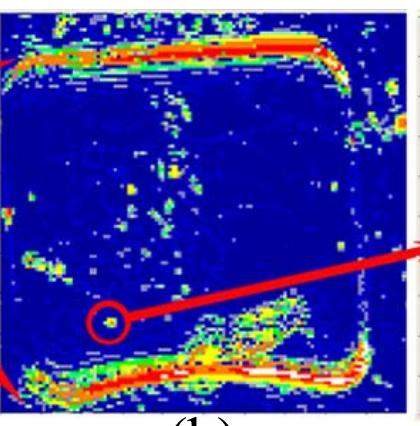

(b)

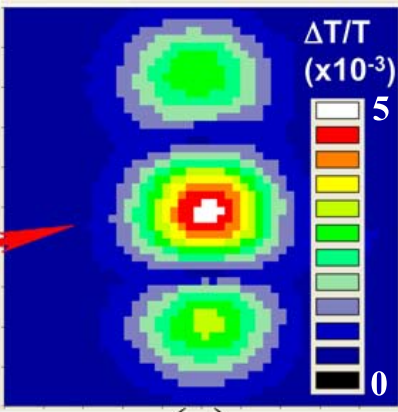

(c)

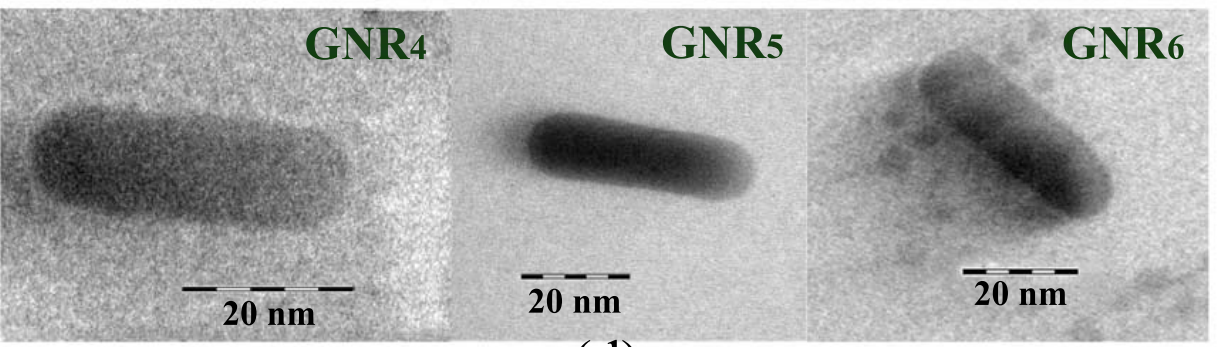

(d)

Figure 3.2: (a) TEM grid showing the $50 \mu \mathrm{m} \times 50 \mu \mathrm{m}$ windows (a silica substrate layer was on top of the windows). The scale bar is $100 \mu \mathrm{m}$. (b) Optical SMS image at $800 \mathrm{~nm}$ wavelength of one of the grid windows showing different objects. (c) Optical SMS magnified view $(1.5 \mu \mathrm{m} \times 1.5 \mu \mathrm{m}$ view $)$ of a gold nanorod. The colour-bar in panel c corresponds to the fractional light transmission change due to extinction by the imaged particle (for both panels b and c). (d) TEM images of the six investigated uncoated and silica-coated gold nanorods. Reprinted with permission from [26]. Copyright 2016 American Chemical Society.

to the modulation direction [108]. When scanning for the location of a particle, a single nanoparticle shows-up as a main peak surrounded by two satellites of opposite sign along the modulation direction (in Figure $3.2 \mathrm{c}$ all the extrema are positive since the absolute value of the transmission change is shown). Measurements of linearly polarized 
spectra were conducted by rotating the light polarization using a quarter-wave plate and a polarizer.

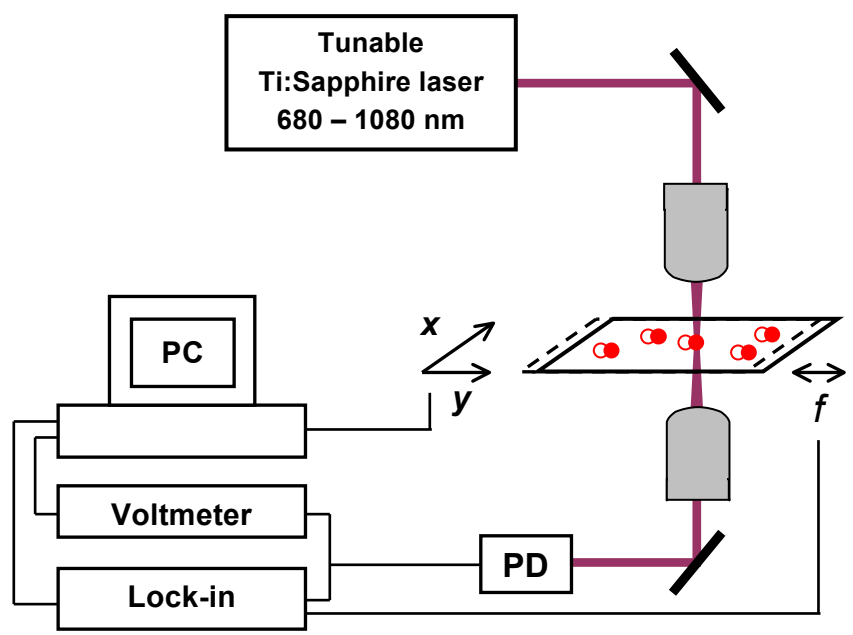

Figure 3.3: The SMS setup showing the laser source injected into the transmission microscope, which consists of a piezo-electric element modulating the sample position at frequency $f$, the $x-y$ scanner, the focusing and collecting microscope objective, the Si photodiode (PD), the digital voltmeter, and the lock-in amplifier demodulating the signal. Reprinted with permission from [26]. Copyright 2016 American Chemical Society.

The optical study was performed prior the TEM measurements to avoid any influence of substrate or nanoparticle modification by the electron beam. The optical measurements were carried out by detecting the nanoparticle with an SMS optical image with unpolarized light at $800 \mathrm{~nm}$ (which is near the longitudinal SPR wavelength of the synthesized nanorods). After locating light responding objects, the sensitivity of the signal amplitude to light polarization was checked to confirm its possible assignment as a single nanorod (for a single GNR there is a very large contrast in the extinction for two orthogonal directions of light polarization around the SPR) [103, 137]. Its spectrum around the longitudinal SPR was then measured for light polarization yielding the maximum along the long axis of the nanoparticle (i.e., the direction maximizing the signal amplitude). The spatial coordinates of its location were thus determined for the follow-up TEM characterization of its geometry and dimensions. TEM images of the six rods are shown in Figure 3.2d. 
The extinction spectra of three silica-coated $\left(\mathrm{S}-\mathrm{GNR}_{1}, \mathrm{~S}-\mathrm{GNR}_{2}\right.$, and $\left.\mathrm{S}-\mathrm{GNR}_{3}\right)$ and three uncoated GNRs $\left(\mathrm{GNR}_{4}, \mathrm{GNR}_{5}\right.$ and $\left.\mathrm{GNR}_{6}\right)$, spin-coated onto a TEM grid covered by a $40 \mathrm{~nm}$ thick silica support substrate (Figure $3.2 \mathrm{a}$ ), were measured using SMS (Figure $3.2 \mathrm{~b}, \mathrm{c}$ ). The individual nanorods were detected by demodulating the power of the transmitted light at twice the modulation frequency of the nanoparticle position. A single particle then shows up as a main peak surrounded by two satellites in the direction of the spatial modulation (Figure 3.2.). For the investigated GNR sizes, absorption dominates over scattering so that the extinction cross sections are almost identical to the absorption ones. TEM images of the same six nanorods were recorded (Figure $3.2 \mathrm{~d}$ ). The dimensions estimated from the TEM images are given in Table 3.1. The uncertainty in the TEM dimension measurements was approximately $\pm 0.5 \mathrm{~nm}$.

Table 3.1: Dimensions of the silica-coated and bare GNRs used in the models ${ }^{a}$.

\begin{tabular}{|c|c|c|c|c|c|}
\hline Rod & $l_{\mathrm{e}} / l_{\mathrm{o}}(\mathrm{nm})$ & $w_{\mathrm{e}} / w_{\mathrm{o}}(\mathrm{nm})$ & $a_{\mathrm{e}} / a_{\mathrm{o}}$ & $s_{\text {tip }}(\mathrm{nm})$ & $s_{\text {mid }}(\mathrm{nm})$ \\
\hline \hline $\mathrm{S}_{-\mathrm{GNR}_{1}}$ & $33.0 / 33.4$ & $8.8 / 8.8$ & $3.8 / 3.8$ & 9.5 & 15.7 \\
\hline $\mathrm{S}_{-\mathrm{GNR}_{2}}$ & $33.9 / 34.0$ & $9.3 / 9.0$ & $3.6 / 3.8$ & 11.3 & 15.8 \\
\hline $\mathrm{S}_{-\mathrm{GNR}_{3}}$ & $32.4 / 31.8$ & $8.8 / 8.2$ & $3.7 / 3.9$ & 8.2 & 9.5 \\
\hline $\mathrm{GNR}_{4}$ & $38.0 /-$ & $11.5 /-$ & $3.3 /-$ & & \\
\hline $\mathrm{GNR}_{5}$ & $40.5 /-$ & $10.8 /-$ & $3.8 /-$ & & \\
\hline $\mathrm{GNR}_{6}$ & $39.0 /-$ & $12.0 /-$ & $3.3 /-$ & & \\
\hline
\end{tabular}

${ }^{a} l_{\mathrm{e}}, w_{\mathrm{e}}$ and $a_{\mathrm{e}}$ are the length, width, and aspect ratio of the nanorods deduced from TEM imaging, respectively. $l_{\mathrm{o}}, w_{\mathrm{o}}$ and $a_{\mathrm{o}}$ are the refined dimensions deduced from fitting the theoretical model to the optical extinction spectra. $s_{\text {tip }}$ and $s_{\text {mid }}$ are the silica shell thickness on the tip and on the side of the GNR, respectively (see Figure 3.4b. It was not possible to deduce refined optical dimensions for the bare GNRs.

\subsubsection{Finite element model}

A finite element model was built using a commercially available finite element software, COMSOL Multiphysics 4.2 (COMSOL Inc., Burlington, MA), taking into account the dimensions of GNRs from TEM measurements and a realistic geometry of the SMS setup that included the TEM grid silica substrate (Figure 3.4. The incident electric field, $\mathbf{E}_{\mathrm{inc}}$, 
propagated in the downward direction (relative to 3.4) with a polarization in the left/right direction (along the longitudinal axis of the nanorod).

Although the experiment consisted of an unbounded domain, the computational model had to be artificially truncated using appropriate boundary conditions in all directions (see Figure 3.4). Ideally boundary conditions such as an ABC and/or a PML would be used to minimize the effect of the artificial boundaries. Initial testing using combined $\mathrm{ABC} / \mathrm{PML}$ boundaries to truncate computational domain produced good results in the simulations of the electromagnetic wave propagation in a homogeneous environment, but led to artifacts in simulations where the substrate was adjacent to the boundary. PEC boundaries, $\mathbf{n} \times \mathbf{E}=0$, and PMC boundaries, $\mathbf{n} \times \mathbf{H}=0$, which simulate periodic structures when the boundaries are perpendicular to the incident electric and magnetic fields, respectively, were used in place of absorbing boundaries. The PEC/PMC boundaries were placed sufficiently far from the GNR such that the distance between the periodic GNR structures were large enough to prevent coupling between them. The PEC and PMC boundary conditions were used at the boundaries perpendicular and parallel to the incident electric field polarization, respectively. The top and the bottom boundaries of the model had an ABC imposed on them using a Sommerfeld radiation condition [138]. The $\mathrm{ABC}$ was used so that the boundary appears transparent to the incident radiation and absorbs the scattered field. All internal boundaries had tangential E-field continuity (ensured by the use of vector basis functions [95]). The dimensions of the computational domain were chosen so that any further increase in the domain size did not alter the solution. This ensured that all the artificial external boundaries did not introduce artifacts into the model.

The refractive index of the nanorod's silica shell and the TEM silica substrate were set to a constant value of $n=1.46$ [139]. As usually done for metal nanoparticles, the dielectric function of the gold part, $\tilde{\varepsilon}_{\mathrm{GNR}}$, was obtained from the bulk one, $\tilde{\varepsilon}_{\mathrm{B}}$ [115], and corrected for intrinsic size effects. For the sizes investigated here, the intrinsic properties related to GNR size were included by modifying the electron scattering rate $\gamma_{\mathrm{D}}$ of the conduction electrons in the Drude term of the gold dielectric function to take into account additional electron scattering off the nanoparticle's surface [44]. For confined electrons 
it can be written as

$$
\tilde{\varepsilon}_{\mathrm{GNR}}=\tilde{\varepsilon}_{\mathrm{B}}+\frac{\omega_{p}^{2}}{\omega^{2}+i \omega \gamma_{\mathrm{D}_{0}}}-\frac{\omega_{p}^{2}}{\omega^{2}+i \omega\left(\gamma_{\mathrm{D}_{0}}+\frac{A v_{\mathrm{F}}}{L_{\mathrm{eff}}}\right)}
$$

where $\gamma_{\mathrm{D}_{0}}$ is the bulk like electron scattering rate in the particle and $v_{\mathrm{F}}$ the electron Fermi velocity. $A$ is a dimensionless parameter representing details of the electron interactions with the confining surface, and $L_{\text {eff }}$ is an effective mean free path of the surface confined electrons. $L_{\text {eff }}$ corresponds to the particle diameter for spherical nanoparticles [44, 140, 141], while for nonspherical objects its appropriate dimension dependence remains an ongoing area of investigation. A simple billiard type model produced the relationship, $L_{\text {eff }}=\frac{4 V}{S}$, where $V$ is the volume and $S$ is the surface area of the object [142]. Note that $L_{\text {eff }}$ varies weakly for the investigated particles. A different dependence on their dimensions only leads to different $A$ values when reproducing the measured spectra.

The surface confinement broadening parameter was assumed to have a constant value of $A=0.5$. This reproduced the longitudinal SPR full-width at half-maximum (fwhm) of the three silica-coated GNRs to within $1 \%$ and the fwhm of the simulated bare rods were $5 \%$ to $10 \%$ lower than the measured fwhm. Since this value was used for nanorods of very similar sizes, the dependency of the fwhm on the mean free path of the conduction electrons, $L_{\text {eff }}$ (equation 3.1) was not tested. In addition, the imaginary part of the bulk gold dielectric function [115] has been suspected of being inaccurate in the spectral range used in the experiments [104], complicating the quantitative estimation of $A$ from measured spectra. Though the assumed $A$ value is consistent with a previous investigation [143], a more reliable determination requires investigation of the fwhm as a function of the nanorod dimensions [143] in a controlled environment, which would reduce chemical damping effects due to surface bound molecules, as was recently done for nanospheres [140].

Meshing was performed with tetrahedral elements used with quadrilateral vector basis functions [95]. The resulting mesh had approximately 370000 to 540000 elements, resulting in approximately $2-4$ million degrees of freedom. After computation of the electromagnetic field, the absorption cross-section $\sigma_{\text {abs }}$ of a nanorod was obtained by integrating the absorbed power density over the volume $V$ of its gold part divided by the 
incident light intensity:

$$
\sigma_{\mathrm{abs}}(\omega)=\frac{2}{n c_{0} \varepsilon_{0}\left|\mathbf{E}_{\mathrm{inc}}\right|^{2}} \int_{V} \Re\left\{\sigma(\omega) \mathbf{E} \cdot \mathbf{E}^{*}-i \omega \mathbf{E} \cdot \mathbf{D}^{*}\right\} \mathrm{d} V
$$

where $\mathbf{E}$ and $\mathbf{D}$ are the electric and displacement field at the frequency $\omega$, respectively, and $\sigma=\omega \Im\left\{\tilde{\varepsilon}_{\mathrm{GNR}}\right\}$ is the conductivity of the gold nanorod. The $\Re\{\}$ and $\Im\{\}$ operators produce the real and imaginary components of the complex integrand, respectively. The scattering cross-section $\sigma_{\text {sca }}$ was calculated from the outgoing electromagnetic energy flux over a surface $S$ surrounding the gold nanorod:

$$
\sigma_{\text {sca }}(\omega)=\frac{2}{n c_{0} \varepsilon_{0}\left|\mathbf{E}_{\mathrm{inc}}\right|^{2}} \oint_{S} \Re\left\{\mathbf{E}_{\mathrm{sca}} \times \mathbf{H}_{\mathrm{sca}}^{*}\right\} \mathrm{d} S
$$

where $\mathbf{E}_{\text {sca }}$ and $\mathbf{H}_{\text {sca }}$ are the scattered electric and magnetic fields at the frequency $\omega$, respectively.

The extinction cross-section $\sigma_{\text {ext }}$ was then obtained as the sum of absorption and scattering: $\sigma_{\text {ext }}=\sigma_{\text {abs }}+\sigma_{\text {sca }}$. For the small-size bare or silica-coated nanorods investigated here, absorption dominates over scattering (simulations showed that $\sigma_{\text {sca }}\left(\lambda_{\mathrm{R}}\right) / \sigma_{\text {abs }}\left(\lambda_{\mathrm{R}}\right)<$ $0.01)$ so that $\sigma_{\text {ext }}=\sigma_{\text {abs }}$ was assumed. The effect of light reflected by the TEM grid substrate during the SMS measurements of $\sigma_{\text {ext }}$ was removed by a normalizing against a transmission measurement through a section of the substrate where no GNRs were present. Since the computational model calculated $\sigma_{\text {abs }}$ which was assumed to be equal to $\sigma_{\text {ext }}$, incident light reflected by the silica substrate had no effect on the theoretical $\sigma_{\text {ext }}$ calculation.

On the basis of these TEM images, a detailed geometric model was created to represent the gold-silica boundary and outer silica surface of the GNR by using cylinders capped with hemispheres, as shown in Figure 3.4. To allow for nonuniform silica coating thickness around each GNR, the lengths of the cylindrical section of the gold part and of the cylindrical section of the silica coating were not assumed to be identical. The FEM model was used to simulate the extinction spectra of these GNRs under the experimental conditions. Figure 4.6 provides an example of the surface mesh discretization of the GNR, and substrate, and the amplitude of the total electric field profile around the GNR (of the incident plane wave and of the field scattered by the GNR).

Table 3.2 contains parameters and values that were used in the EM model in this 
thesis.

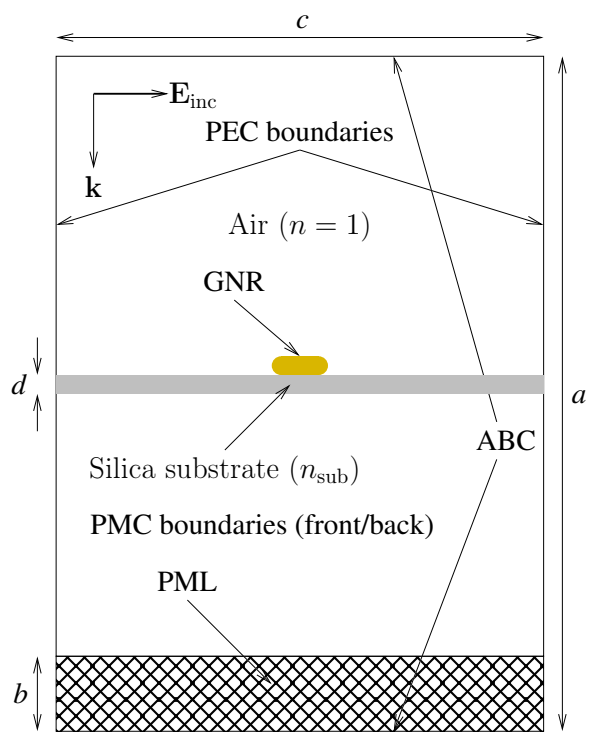

(a) Computational geometry.

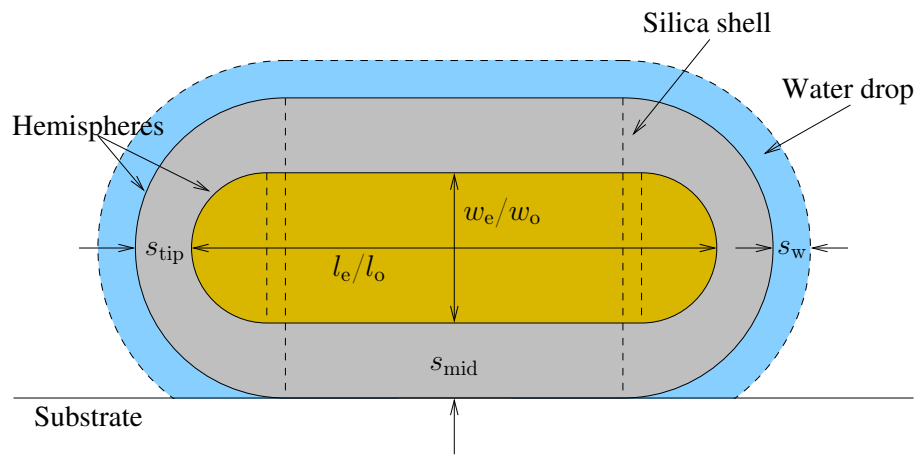

(b) Details of GNR geometry.

Figure 3.4: Schematics of the model geometry (not shown to scale). (a) $2 \mathrm{D}$ crosssection representation of the 3D geometry and boundary conditions of the computational model. The dimensions were truncated at the top and bottom using an $\mathrm{ABC}$, at the left and right with a PEC surface, and at the front and back with a PMC surface. The incident wave was polarized in the left-right direction, travelling downward. Therefore an additional absorbing layer, known as a PML was placed at the bottom of the domain. The medium inside the domain was air, and the GNR support layer on the TEM grid was modelled as a $40 \mathrm{~nm}$ thick silica substrate. In the computational model $a=2400 \mathrm{~nm}, b=400 \mathrm{~nm}, c=1400 \mathrm{~nm}$, and $d=40 \mathrm{~nm}$. The domain in the front-to-back direction had a size equal to $c$; (b) Morphology of a GNR used in the model, which assumed cylindrical shape with hemispherical endtips for both the gold part and the outer silica surface. The various parameters used to define its size are also shown. Reprinted with permission from [26]. Copyright 2016 American Chemical Society. 
Table 3.2: Parameters that were used in the electromagnetic model.

\begin{tabular}{|c|c|c|}
\hline Symbol & Value/Expression & Description \\
\hline$\lambda$ & & Laser wavelength \\
\hline$c_{0}$ & $299792458 \mathrm{~m} / \mathrm{s}$ & Speed of light in vacuum \\
\hline$\omega$ & $2 \pi c_{0} / \lambda$ & Angular frequency \\
\hline$\varepsilon_{0}$ & $8.854187817 \ldots \times 10^{-12} \mathrm{~F} / \mathrm{m}$ & Vacuum permittivity \\
\hline$\tilde{\varepsilon}$ & eq. 2.4 & Size-dependant dielectric constant [26] \\
\hline $\mathbf{E}$ & eq. $\overline{2.2}$ & Electric field strength \\
\hline$E_{0}$ & $\sqrt{\frac{2 I_{\text {tot }}}{c_{0} n \varepsilon_{0}}}$ & Incident electric field \\
\hline$f\left(\tau_{\mathrm{w}}\right)$ & $\frac{2.35482}{\tau_{\tau}} e^{\frac{-\left(t-\tau_{\mathrm{W}}\right)^{2}}{2(2.35482)^{2}}}$ & Gaussian shape pulse function \\
\hline$\tau_{\mathrm{w}}$ & 60 fs or $6 \mathrm{ps}$ & Laser pulse at full width at half maximum \\
\hline$I_{\text {tot }}$ & $0.5 c_{0} n \varepsilon_{0}\left|E_{0}^{2}\right|$ & Incident irradiance of laser light \\
\hline$e$ & $1.602176565(35) \times 10^{-19} \mathrm{C}$ & Electron charge \\
\hline$\rho_{\mathrm{e}}$ & & Density of free electron plasma \\
\hline$m_{\mathrm{e}}$ & $9.10938291(40) \times 10^{-31} \mathrm{~kg}$ & Electron mass \\
\hline$\tilde{\varepsilon}_{\text {bulk }}$ & see Johnson and Christy [115] & Bulk dielectric function \\
\hline$\mu_{0}$ & $4 \pi \times 10^{-7} \mathrm{~N} / \mathrm{A}^{2}$ & Magnetic permeability in vacuum \\
\hline$\omega_{p}$ & $\sqrt{\frac{\rho_{\mathrm{e}} e^{2}}{\varepsilon_{0} m_{\mathrm{e}}}}$ & Plasma frequency of free electron gas \\
\hline$\gamma_{\mathrm{D} 0}$ & $1.094 \times 10^{14} 1 / \mathrm{s}$ & Bulk relaxation frequency 115 \\
\hline$v_{\mathrm{F}}$ & $1.41 \mathrm{~nm} \mathrm{fs}^{-1}$ & Fermi velocity 44 \\
\hline$L_{\text {eff }}$ & $4 V_{\mathrm{au}} / S_{\mathrm{au}}$ & Reduced effective mean free path length [142] \\
\hline$V_{\mathrm{au}}$ & & Volume of the particle \\
\hline$S_{\mathrm{au}}$ & & Surface area of the particle \\
\hline$\eta$ & $(5.5 \pm 1.5) \times 10^{-7} \mathrm{fs}^{-1} \mathrm{~nm}^{-3}$ & Radiation damping proportionality constant 92 ] \\
\hline$A$ & 0.5 & Broadening parameter [26] \\
\hline$\sigma_{\text {abs }}$ & $\frac{1}{I_{\mathrm{tot}}} \int_{V_{\mathrm{au}}} \Re\left(Q_{\mathrm{rh}}\right) d V_{\mathrm{au}}$ & Absorption cross-section of the gold nanoparticle [117] \\
\hline$n$ & & Refractive index of water [144] \\
\hline $\mathrm{D}$ & & Dielectric displacement \\
\hline$\sigma$ & & Electric conductivity \\
\hline$Q_{\mathrm{rh}}$ & see eq. 2.10 & Resistive losses during laser pulse interaction [117] \\
\hline
\end{tabular}




\subsection{The role of gold nanoparticle morphology and cou- pling in optical breakdown during picosecond pulse exposures}

In order to analyze the role of nanoparticles' morphology on LIOB a fully coupled theoretical model of optical breakdown was developed. A comparison of on- and off-resonance 6 ps laser pulse interactions with uncoupled and tightly-coupled gold nanospheres and nanorod monomers of different sizes was done, with a focus on the thermal and optical processes. As illustrated in Figure 3.5, the FE model combined an electromagnetic field interaction with gold nanoparticle that includes the surface confinement corrections to the bulk optical properties of gold [26]; with a hyperbolic two-temperature model (TTM) for the thermodynamic evolution of gold nanoparticle electron and lattice temperatures [113; with the rate equation of free electron plasma generation in an aqueous environment based on the Keldysh theory of multiphoton ionization (MPI), the tunnel effect, the avalanche ionization (AI) and thermal ionization (TI) of water [59]; and a photo-thermal emission (PTE) of hot electrons off a gold nanoparticle surface [61].

This section provides the details of LIOB modelling and the parameters that were used to implement the theory of plasma generation given in Section 2.4 .

\subsubsection{The electromagnetic model for LIOB}

The electromagnetic field was calculated using the homogeneous Helmholtz wave equation in all domains (see Figure 3.5). The incident electric field was linearly polarized along the longest axis of the nanostructure ( $y$-axis) with the propagation parallel to the positive $z$ axis. Perfect magnetic and perfect electric conductor boundaries as well as an absorbing boundary condition using PMLs were used to reduce and truncate the geometry [117]. The bulk properties of the medium in the EM domain, were set with refractive index of 1.4 [145] to mimic the optical properties of tissue, which varies from 1.34 to 1.55 [145 147]. The refractive index of the immediate vicinity of the nanoparticles can also significantly alter its optical behaviour, and polymers or intracellular molecules can bind to the nanoparticles, altering the refractive index surrounding the particles. Since this produces uncertainty in the refractive index of the immediate vicinity of the nanoparticles, 


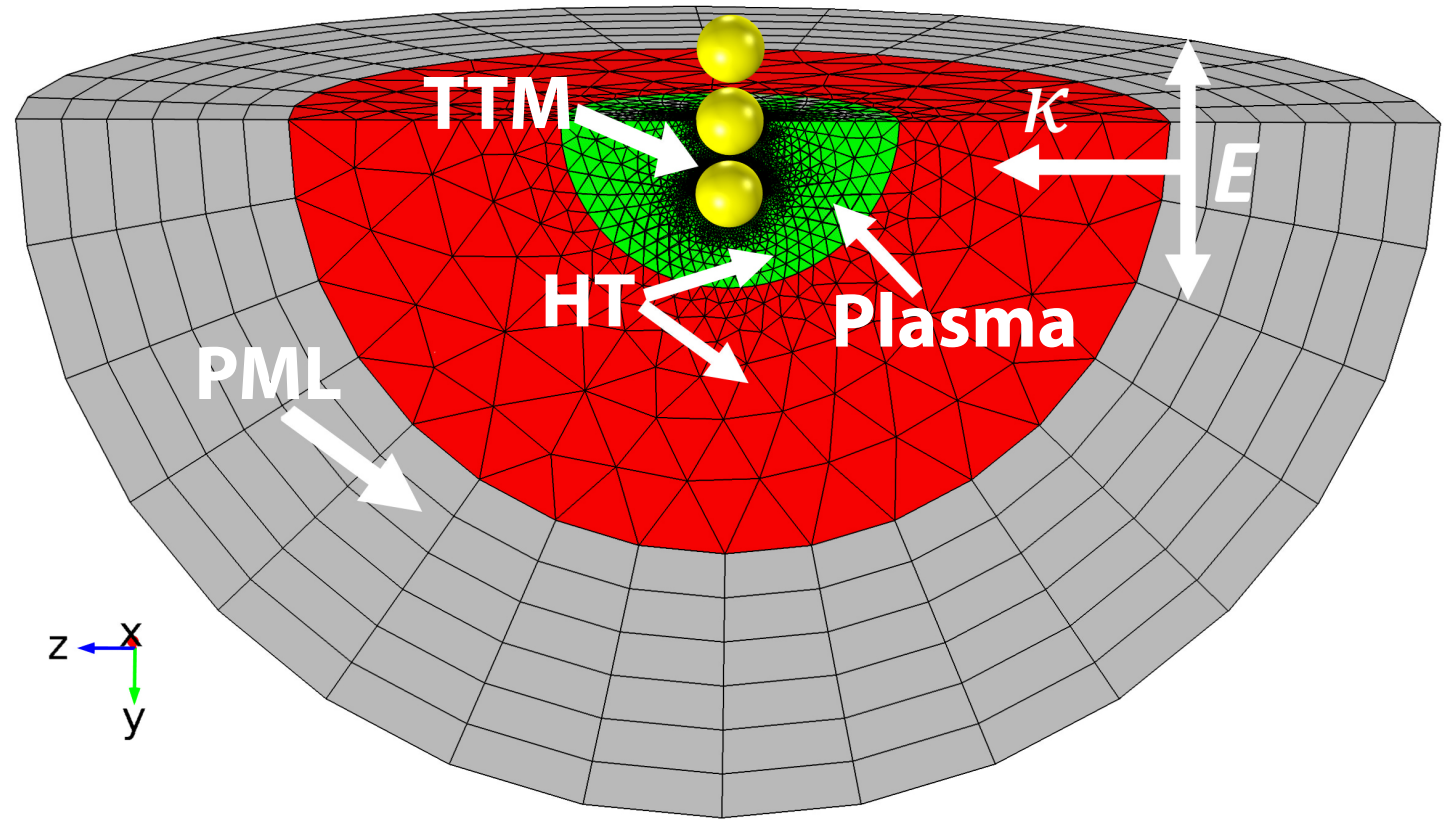

Figure 3.5: Schematic of the model geometry for a nanosphere trimer. The model contains three concentric domains. The most outer domain (grey) represents a combination of PML domain and an absorbing boundary condition to truncate the electromagnetic domain and reduce reflections from artificial boundaries. The red and green domains represent an aqueous environment with a refractive index of 1.4 and physical properties of water. The green domain is used to calculate the plasma formation, and the green and red domains are used to calculate the heat transfer in the medium. The yellow domain shows the gold nanostructure geometry. The incident electric field is linearly polarized along nanosphere trimer length ( $y$-axis) and propagates parallel to positive $z$-axis.

the effect of changes in the optical properties of the immediate environment in the vicinity of the nanoparticles was tested by adding a $2 \mathrm{~nm}$ shell with a refractive index of 1.6.

The dielectric function of medium in the close proximity of the nanostructure were modelled using the Drude formalism in order to account for the shielding of the nanostructure from the incident irradiation:

$$
\varepsilon_{\mathrm{w}}=\varepsilon_{\infty}-\frac{\rho_{\mathrm{e}} e^{2}}{\varepsilon_{0} m^{\prime}\left(\omega^{2}+j \omega / \tau\right)},
$$


$\varepsilon_{\infty}$ is the relative permittivity of the medium (assuming a biologically relevant refractive index of 1.4), $m^{\prime}$ is the electron's reduced mass and $\tau$ is the mean free time between electron/molecule collisions. The optical properties of gold with size corrections to the bulk dielectric function were used [26] since the size corrections to the bulk dielectric function of gold can significantly alter the near-field enhancement and absorption crosssection of nanoparticles with diameter smaller than $20 \mathrm{~nm}$.

\subsubsection{A two-temperature model (TTM) of nanoparticle heating}

A hyperbolic two temperature model for the evolution of the electronic and lattice temperatures of gold nanoparticles and the finite heat diffusion at the gold-medium interface during a laser pulse was solved using the parameters given by Chen, J. K. and Beraun, J. E. [113]. The TTM was coupled to the EM model through the resistive losses, $Q_{\mathrm{rh}}$, during the laser pulse interaction with the gold nanostructure [117].

\subsubsection{Heat transfer (HT) in the medium}

The temperature produced by heat sources due to plasma formation [59], laser pulse interaction and thermal diffusion was solved in all domains outside of the nanoparticles except the PMLs (Figure 3.5. The HT model was coupled to the TTM using the heat diffusion from the gold lattice to the surrounding medium through interface conductance, $Q_{\mathrm{au} \mid \mathrm{w}}[118]$.

\subsubsection{Plasma dynamics}

The dynamics of plasma formation was calculated in a finite spherical domain surrounding the nanoparticles (Figure 3.5). The plasma rate equation [59], based on the full Keldysh theory for MPI [114], the tunnelling effect, AI, TI [59, 66], diffusion and recombination losses and PTE [61] of hot electrons from the gold surface, was solved to determine the dynamics of the free electron plasma density in the vicinity of the nanoparticle. The parameters for plasma theory are those described by Linz et al. and Bulgakova et al. The plasma dynamics model was coupled to the EM model through the electric field, and the changes in the dielectric function of the environment due to the free electron plasma formation. The temperature rise of the conduction electrons in the gold from the TTM 


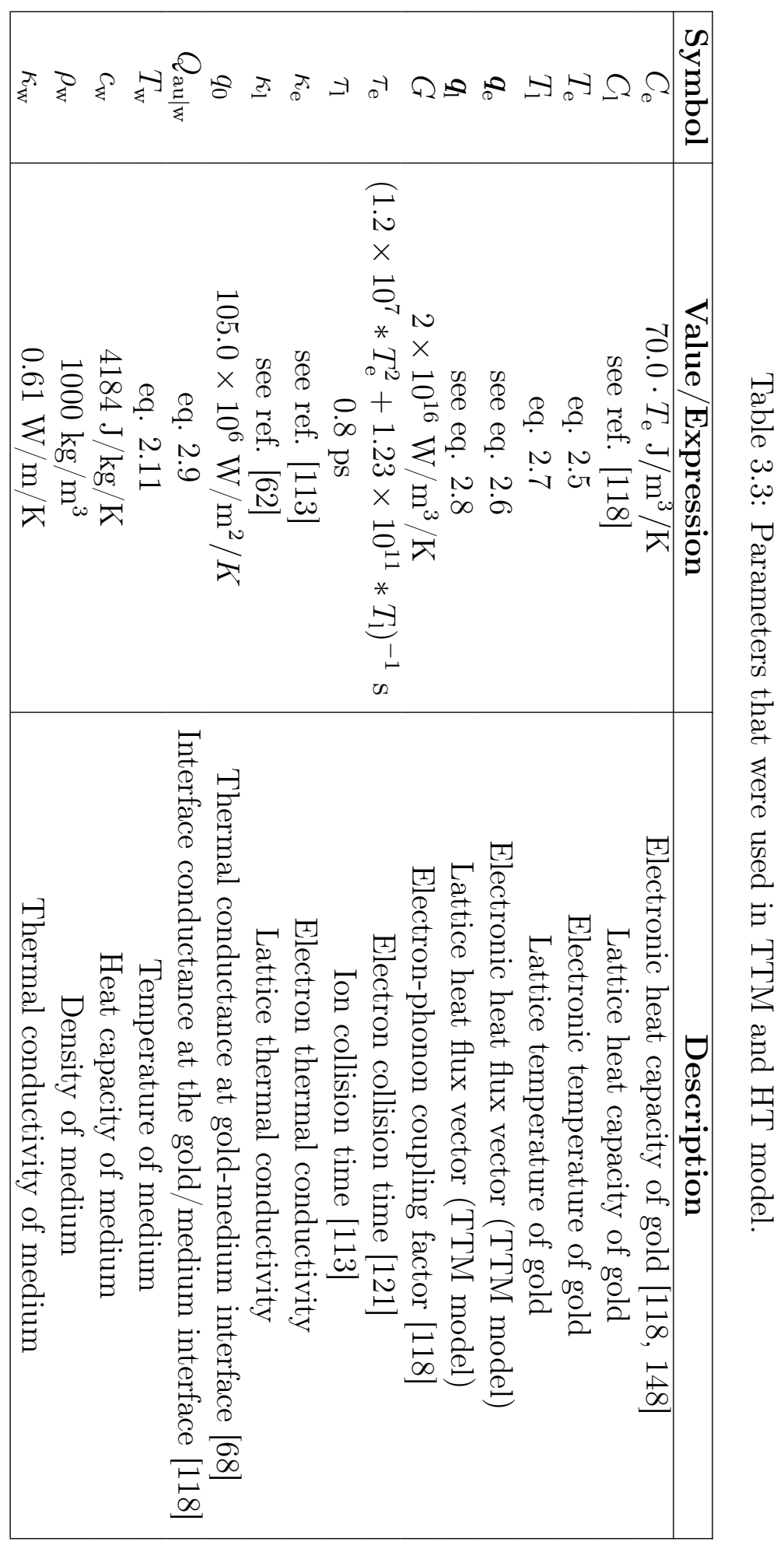


were used to couple TTM to the PTE of hot electrons. The HT model was coupled to PTE of plasma dynamics model through the temperature rise in the medium.

These models were implemented and coupled together using the commercially available finite element package, COMSOL Multiphysics version 4.4. Assemblies of particles were spaced $4 \mathrm{~nm}$ apart in order to account for separation by surfactant or coupling molecules on the particles surface while still keeping a strong plasmonic coupling effect [83]. The 3D geometry was reduced to one quarter of the full geometry (see Figure 3.5) by utilizing symmetry planes and absorbing boundary conditions. PEC and PMC conductor boundaries are used to truncate domain to one quarter of full 3D in EM model. The TTM, plasma and heat transfer domains were meshed using tetrahedral elements with quadrilateral vector basis function (see Figure 3.5). Swept meshing was used for the PML domain (see Figure 3.5). The maximum mesh element size was kept below $1 / 10$ th of the incident wavelength in medium with at least ten times finer elements in the plasma and TTM domains (Figure 3.5. An iterative geometric multigrid solver was used to solve for the electric field. The direct PARDISO solver with a Nested dissection multithreaded algorithm was used for all other models. The coupled model was solved using the frequency-transient stepping with a second order backward differentiation after applying a global scaling, with a tolerance of 0.001 .

\subsection{The wavelength dependence of gold nanorod-mediated optical breakdown with infrared ultrashort pulses}

Several physical phenomena need to be fully coupled in order to fully characterize a low density plasma formation in the vicinity of gold nanorod in water. To do that the model that was developed for investigating the role of nanoparticle morphology on the optical breakdown (Section 3.3) was used and updated to account for the band structure of water in accordance to the latest findings by Linz et al. [74], as described in Section 2.4.6. A schematic of the physical phenomena involved in free electron plasma generation in the vicinity of a gold nanorod and its coupling variables are depicted in Figure 3.1 .

A 3D computational model was built using the commercially available finite element package COMSOL Multiphysics, version 5.1. The finite element model included EM wave propagation, a hyperbolic TTM of finite speed heat diffusion inside a gold nanorod, 


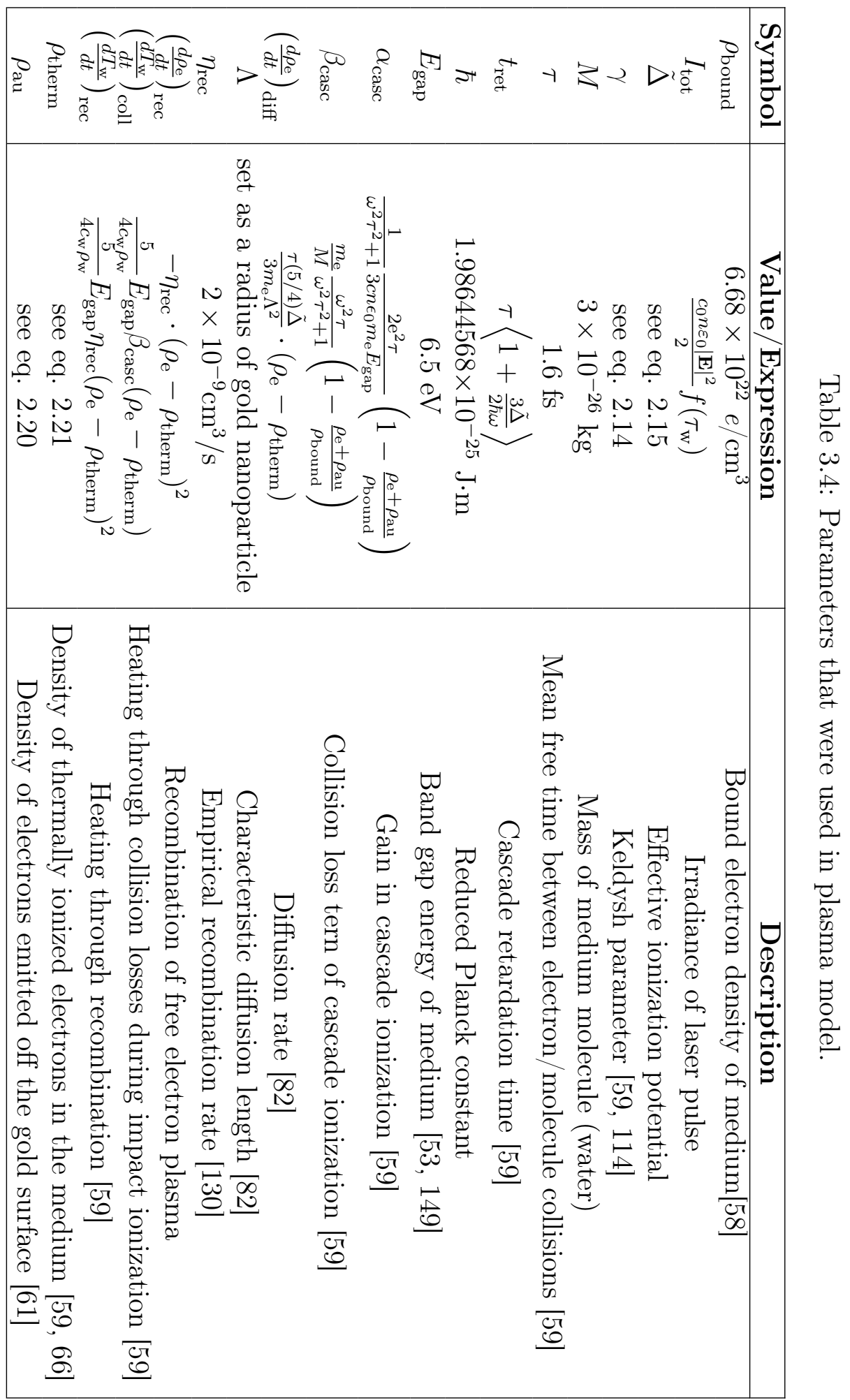




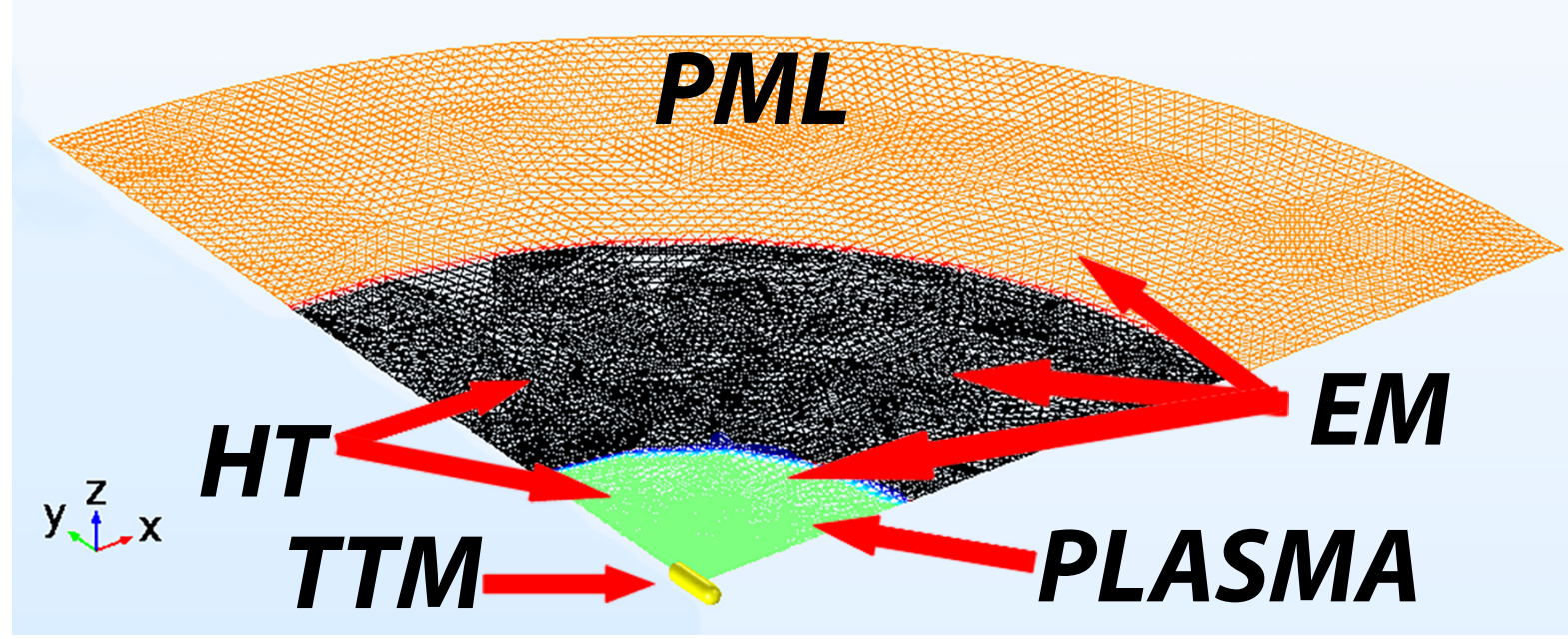

Figure 3.6: Schematic of the model geometry for gold nanorod. The model contains three concentric domains. The most outer domain (orange) represents a combination of PML domain and an absorbing boundary condition to truncate the electromagnetic domain and reduce reflections from artificial boundaries. The green and black domains represent a water domain. The green domain was used to model the plasma formation, and the green and black domains were used to calculate the HT in the medium. The yellow domain shows the gold nanorod geometry (shown as a full 3D geometry for illustration only). Incident electric field is linearly polarized along nanorod length ( $y$-axis) and propagates parallel to positive z-axis. The tetrahedral elements with quadrilateral vector basis function were used to mesh all domains.

$\mathrm{HT}$ in water and a rate equation for plasma formation (Figure 3.1). An artificial outer boundary was used to create a finite sized region of EM wave propagation in order to reduce reflections from the artificial boundary. In addition, the 3D domain was reduced to one-quarter of its volume by using PEC and PMC boundaries for the EM model, and symmetry planes for the rest of the physics models. The schematic of the finite element geometry is shown as a cross-section of the one-quarter geometry in Figure 3.6. The EM model was solved everywhere; the TTM was solved in the gold nanorod; the plasma model was solved in the green coloured domain; and the HT model was solved in green and black coloured domains of Figure 3.6. All domains were meshed using tetrahedral elements with quadrilateral vector basis function. The coupled model was solved using the frequency for the EM model and time domain for the other models. 


\subsection{Validation of the full model}

The model of nanoparticle-mediated optical breakdown was validated in three stages. An EM model of the laser pulse interaction with the nanoparticle. TTM of nanoparticle's electronic and lattice temperatures increase due to absorption of the laser irradiation. A plasma generation model for optical breakdown.

First, the EM part of the model was tested against an analytical Mie solution of Maxwell's equations for a gold nanosphere in a homogeneous environment. The comparison of Mie solution against FE solution helped to validate the COMSOL implementation and meshing parameters of the model. Then the EM model was used to compute the optical spectra of two silica core gold nanoshells, NS660 and NS800, that had been studied recently by Lachaine et al. [97] for use in LIOB and cell perforation. The peak positions of the computed spectra from a single nanoshell were compared with those measured on nanoshell solution by Lachaine et al. [97].

Next, the EM and the two-temperature models were used to calculate the temperature of the gold nanoshell to determine the laser fluence for damage of NS800 nanoshells. Lachaine et al. [97] using TEM, were able to identify the laser fluence threshold that caused cracking and melting of the gold nanoshell.

Finally, the full model with the wavelength dependent properties of water was used to calculate the optical breakdown threshold for both nanoshells, which compared with the threshold of a bubble formation measured by Lachaine et al. [97].

Lachaine et al. [97] used a combination of experimental data and numerical analysis in order to optimize plasmonic nanoparticles for cavitation and cell perforation. To do this, the authors compared experimental and numerical optical breakdown fluence thresholds and thresholds of nanoparticle damage for two gold nanoshells, one with a $78 \mathrm{~nm}$ diameter silica core and a $28 \mathrm{~nm}$ thick gold shell (NS660) and another with a $112 \mathrm{~nm}$ diameter silica core and a $15 \mathrm{~nm}$ thick gold shell (NS800).

Scattering [150] and shadowgraphy [151] techniques were used to image bubble formation and to assess size of the formed bubbles. The bubbles were generated by irradiating a single gold nanoparticle with $70 \mathrm{fs}$ pulses with a $10 \mathrm{~Hz}$ repetition rate at $800 \mathrm{~nm}$. They were able to detect and observe multiple consecutive bubbles for the NS660 nanoshell, while only a single bubble was generated by the NS800 nanoshell. Nanoshell damage was imaged using TEM and a visible-NIR spectrometer was used to detect the plasmon 
resonance shift caused by the damage [97].

For validation the model was modified to include the silica core of the nanoshell. Both gold nanoshells were modelled in a homogeneous water environment with a refractive index of 1.33. The bulk dielectric function of gold was size corrected based on the theory presented in Section 2.1.1, with $A=1.4$ [152], $\Gamma_{\text {bulk }}=73 \mathrm{eV}$ [52], $\Gamma_{\text {rad }}=6.6 \times 10^{-7} \mathrm{eV} / \mathrm{nm}^{-3}$ 52]. For the EM calculation of the spectra (Figure 4.2), the silica core of the nanoshell was modelled with a bulk refractive index of 1.45 , while for the plasma calculation, the refractive index of silica was corrected in accordance with Drude's formalism (see equation 3.4). Free electron plasma generation in silica was modelled with the parameters listed in Table 3.5 .

During a gold nanorod or nanosphere laser pulse interaction, the free electron plasma generation occurs outside the particle (water), while for a gold nanoshell (silica-core and gold shell) free electron plasma can be generated in silica [153, 154] and in water. The optical breakdown in silica (where $\rho_{\mathrm{e}}$ reaches around $1 \times 10^{20} \mathrm{~cm}^{-3}$ to $3 \times 10^{20} \mathrm{~cm}^{-3}$ [155]) can be initiated by MPI and tunneling or through seed electrons emitted from the gold surface (PTE). The process of free electron plasma generation in silica was accounted for in our validation model in order to have a better picture of the overall optical breakdown. This added complexity and non-linearity to the optical breakdown of the modelling process, which resulted in some simulations not running to completion and stopping before solving for the full pulse duration.

Table 3.5: Parameters that were used in the plasma model for silica-core domain.

\begin{tabular}{|c|c|c|}
\hline Symbol & Value $/$ Expression & Description \\
\hline$q_{0}$ & $125 \times 10^{6} \mathrm{~W} / \mathrm{m}^{2} / \mathrm{K}$ & Thermal conductance at gold-silica interface \\
$Q_{\text {au|sio }}$ & eq. 2.9 & Interface conductance at the gold/silica interface \\
$T_{\text {sio }_{2}}$ & eq. 2.11 & Temperature of silica \\
$c_{\text {sio }_{2}}$ & $703 \mathrm{~J} / \mathrm{kg} / \mathrm{K}$ & Heat capacity of silica \\
$\rho_{\text {sio }_{2}}$ & $2203 \mathrm{~kg} / \mathrm{m}^{3}$ & Density of silica \\
$\kappa_{\text {sio }_{2}}$ & $1.38 \mathrm{~W} / \mathrm{m} / \mathrm{K}$ & Thermal conductivity of silica \\
$W_{\text {au }}$ & $3.9 \mathrm{eV}$ & Work function [156] \\
$\rho_{\text {bound }}$ & $2.2 \times 10^{22} e / \mathrm{cm}^{3}$ & Bound electron density of silica [153] \\
$m^{\prime}$ & $0.86 m_{\mathrm{e}}$ & Effective electron mass [157] \\
$M$ & $9.9765 \times 10^{-26} \mathrm{~kg}$ & Mass of silica molecule \\
$\tau$ & $1 \mathrm{fs}$ & Mean free time between electron/molecule collisions [153] \\
$E_{\text {gap }}$ & $9 \mathrm{eV}$ & Band gap energy of silica [153 [155] \\
$\Lambda$ & set as a radius of silica core & Characteristic diffusion length \\
$\tau_{\text {rec }}$ & $150 \mathrm{fs}$ & Characteristic time of electron recombination [158] \\
$n$ & 1.45 & Refractive index of silica \\
\hline
\end{tabular}





\section{Chapter 4}

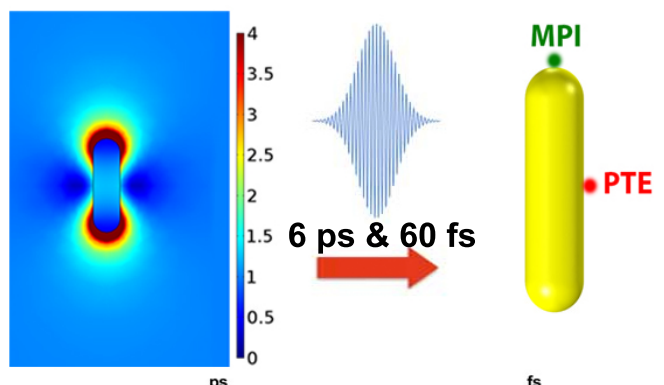

\section{Results}
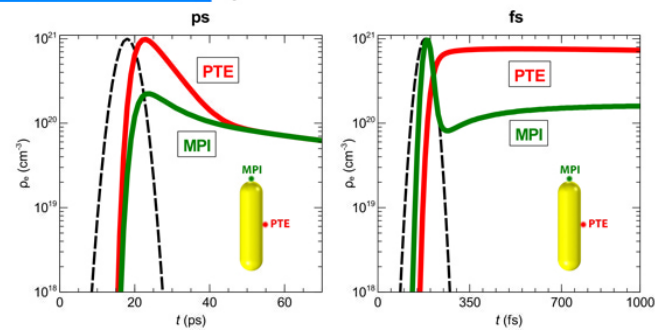

The developed model was validated and used for three studies: (1) the role of the environment on the optical properties of gold nanorods, (2) the role of gold nanoparticle morphology and plasmon coupling on the optical breakdown threshold, and (3) the wavelength dependence of gold nanorod-mediated optical breakdown during picosecond and femtosecond pulses.

\subsection{Validation of the model}

The model validation is divided into three parts: A validation of the EM model alone, a validation of a combined, EM-TTM model and a validation of the full model. In the validation of the full model, the experimental bubble formation fluence threshold from the nanoshells was compared against the prediction by the full model. A better agreement with the NS800 particle than the NS660 particle was found. This resulted in further analysis, which is discussed in Chapter 6 .

\subsubsection{Validation of the EM model}

A finite element model of a $40 \mathrm{~nm}$ diameter gold sphere with bulk dielectric properties from Johnson and Christy [115] was modelled in a homogeneous dielectric environment with refractive index $n=1.33$. A comparison against a Mie solution with the same parameters, is shown in Figure 4.1. As can be seen, the extinction cross-section, $\sigma_{\text {ext }}$, from the COMSOL Multiphysics EM model closely matches the Mie solution. 
In addition to the validation of the EM model against a Mie solution, Section 4.2 shows an excellent quantitative agreement between the modelled and experimentally measured extinction cross-section a the single silica-coated gold nanorod on a dielectric substrate.

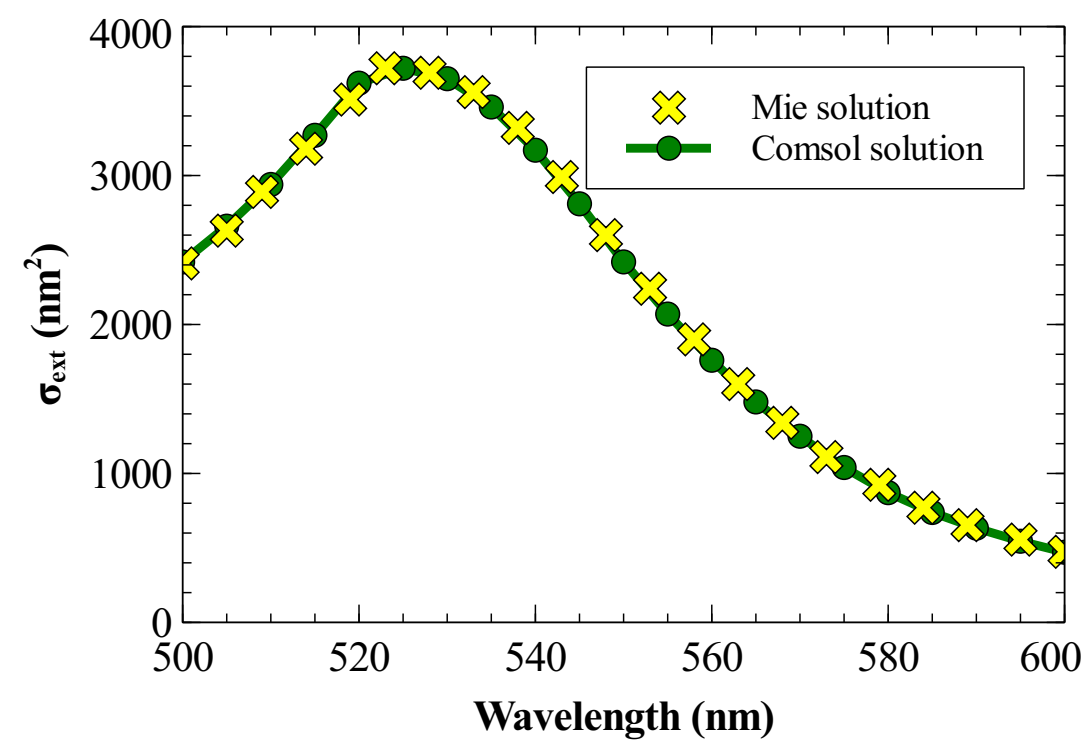

Figure 4.1: Comparison of extinction cross-section, $\sigma_{\text {ext }}$, from the finite element (COMSOL) model and Mie solution for a gold sphere with radius of $20 \mathrm{~nm}$ and bulk dielectric function of gold [115].

Finally, the EM model was validated using reported by Lachaine et al. 97] extinction spectroscopy measurements of two nanoshells (NS660 and NS800 that are described in Section 3.5. Figure 4.2 a, shows the model results for the two nanoshells. These were calculated based on the theory presented in Section 2.1. The model predicted a peak wavelength for the extinction cross-section for NS660 around $660 \mathrm{~nm}$ and for NS800 the peak is located around $760 \mathrm{~nm}$. In case of the NS660 the modelled peak position is in the agreement with the peak position reported for spectra of a colloidal solution NS660 particles by Lachaine et al. [97]. On the other hand, the peak position predicted by the model for NS800 was at $760 \mathrm{~nm}$ in comparison to $780 \mathrm{~nm}$ that was reported for a colloidal solution of particles by Lachaine et al. [98. The disagreement can come from the dispersion of nanoparticles size and/or the presence of surfactant molecules [26] that affects the plasmon peak position. 
a.

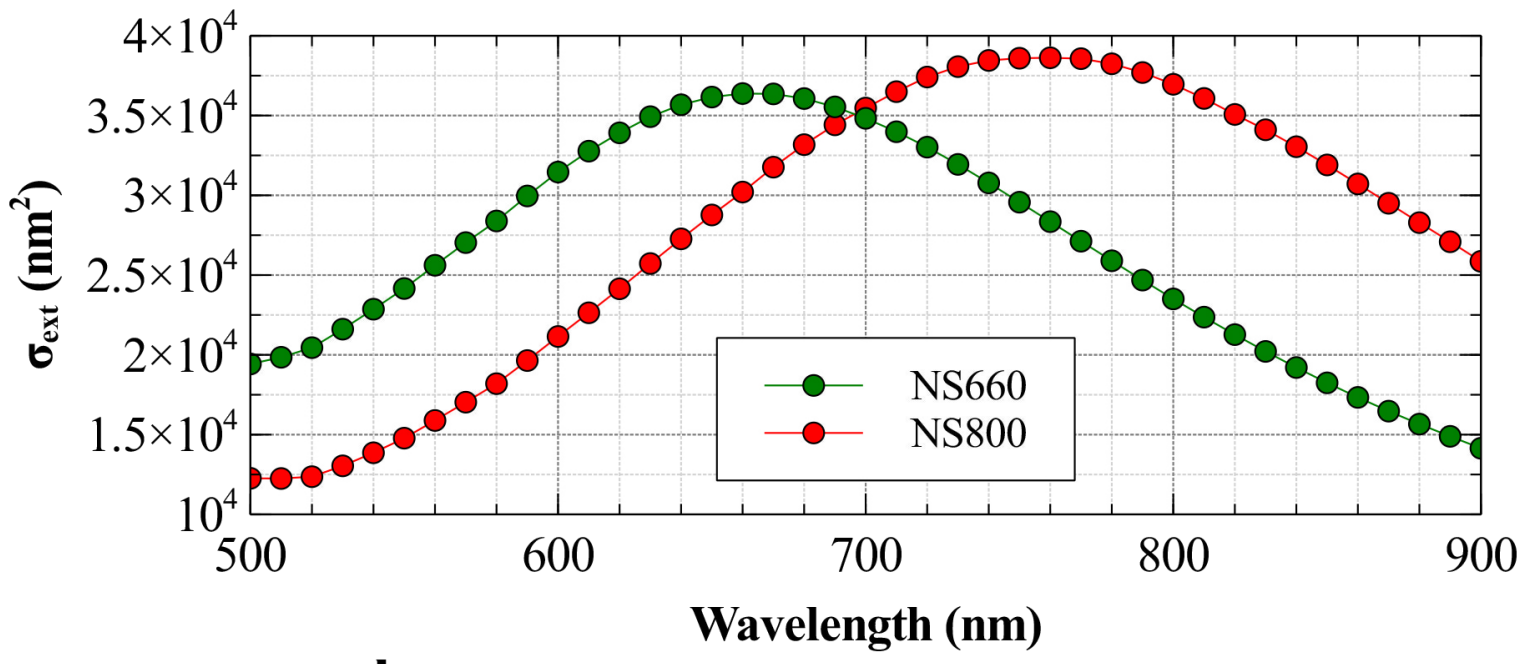

b.

c.

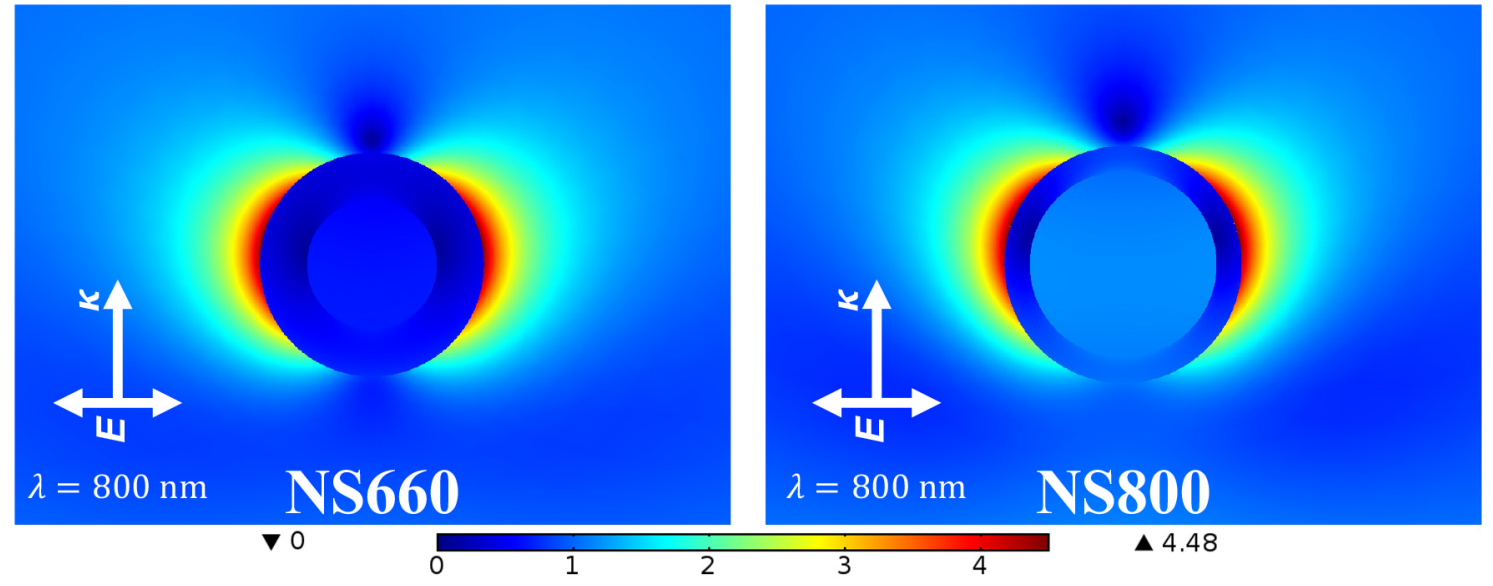

Figure 4.2: a. Extinction cross-section of NS660 (39 nm silica core and $28 \mathrm{~nm}$ gold shell thickness) and NS800 (56 nm silica core and $15 \mathrm{~nm}$ gold shell thickness) nanoshells in homogeneous water environment. b. and c. shows near field enhancement by gold nanoshells exposed at $800 \mathrm{~nm}$ wavelength.

\subsubsection{Validation of the combined EM and TTM model}

Lachaine et al. [97] found that up to 70\% of NS800 particles sustained damage due to cracking and melting of their gold shell when irradiated at $3 \mathrm{~mJ} / \mathrm{cm}^{2}$ fluence (Figure $4.3 \mathrm{a}$ ). Around $98 \%$ of the particles were found either melted or cracked when NS800 were irradiated at $8 \mathrm{~mJ} / \mathrm{cm}^{2}$ fluence. In order to check whether the lattice temperature of the NS800 nanoshell reaches the melting point of gold during a $70 \mathrm{fs}$ pulse exposure, we 
ran a combination of the EM and two-temperature models at fluences in the range of $0.5-8 \mathrm{~mJ} / \mathrm{cm}^{2}$. Figure $4.3 \mathrm{~b}$ shows the maximum lattice temperature, $T_{1, \max }$, that the gold shell reaches after a 70 fs pulse exposure. The EM-TTM model predicted a threshold fluence of $3 \mathrm{~mJ} / \mathrm{cm}^{2}$, above which the gold shell starts to melt and the nanoshell looses its integrity. This threshold fluence is in good agreement with the experimental findings of the particle's damage.

a.

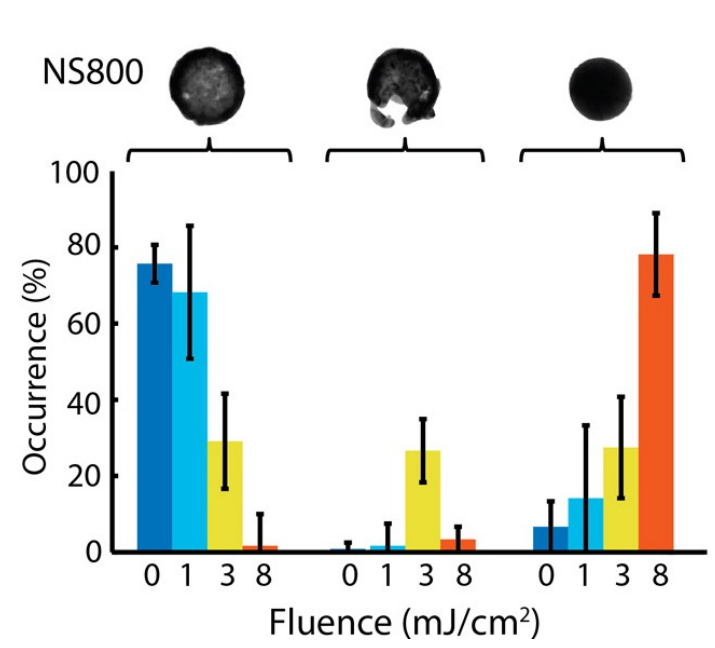

b.

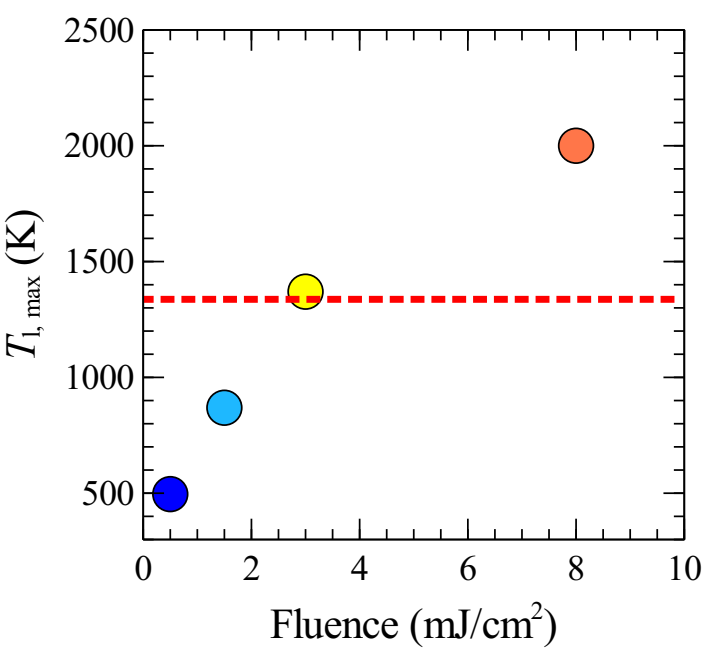

Figure 4.3: a. Experimental data of intact, cracked, and melted of NS800 particles occurrence after laser pulse exposure. Dark blue bars correspond to $0 \mathrm{~mJ} / \mathrm{cm}^{2}$, light blue to $1 \mathrm{~mJ} / \mathrm{cm}^{2}$, yellow to $3 \mathrm{~mJ} / \mathrm{cm}^{2}$, and orange to $8 \mathrm{~mJ} / \mathrm{cm}^{2}$. Reprinted with permission from [97]. Copyright 2016 American Chemical Society. b. Maximum lattice temperature, $T_{1, \max }$, predicted by the EM-TTM model. Red dashed line indicates $1337 \mathrm{~K}$, a melting point of bulk gold.

Although, the combined EM and TTM model correlates well with observed damage of the nanoshell, it should be noted that for the high fluence rates used, a high density of free electron plasma is generated in the vicinity of the nanoparticle. This will have two effects on the temperature in the vicinity of the nanoparticle. First, the high density plasma will absorb the incoming laser radiation, and shielding the gold nanoshell and lowering the nanoparticle's heating. Second, the absorption of the incoming radiation by the high density plasma will heat the water through electron collision and recombination. 
As will be shown in Chapter 6, our model can calculate and predict the temperature of the water that is reached during the optical breakdown process before gold shell of the particle reaches the temperature of the melting point of gold.

\subsubsection{Validation of the full model}

Figure 4.4 shows a comparison of the free electron density predicted by the full model against experimentally measured bubble size [97. The temporal electron density generation in water at the location of the maximum electron density along with the relative temporal shape of the $70 \mathrm{fs}$ laser pulse intensity for different fluences are shown in Figure 4.4 a. Figure $4.4 \mathrm{~b}$ show the bubble diameters and the maximum electron density at various fluences. From Figure $4.4 \mathrm{~b}$ one can see that bubbles started to be detectable in the experiments at a fluence of around $7 \mathrm{~mJ} / \mathrm{cm}^{2}$. At this fluence the model predicts a free electron density of around $1.8 \times 10^{20} \mathrm{~cm}^{-3}$ (through interpolation of the results). Linz et al. [75] observed a threshold of $\rho_{\text {bf }}=1.8 \times 10^{20} \mathrm{~cm}^{-3}$ as a free electron density needed for a bubble formation during optical breakdown experiments in pure water. Thus, our LIOB model described in Chapter 2 was able to predict the experimental results very well.

Due to a good agreement in free electron densities $\left(\rho_{\mathrm{bf}}=1.8 \times 10^{20} \mathrm{~cm}^{-3}\right)$, that were calculated and matched experimental bubble detection in case of a pure water experiments by the Linz et al. [75] and gold nanoshell-mediated bubble generation by Lachaine et al. [97, the same threshold for the bubble formation was used when calculating optical breakdown threshold for NS660 particle.

Lachaine et al. [97] detected bubble formation by NS660 particles using a 70 fs pulse (at $800 \mathrm{~nm}$ wavelength) with fluences above approximately $35 \mathrm{~mJ} / \mathrm{cm}^{2}$ (see Figure 4.5). In this case the model of the NS660 nanoshell predicted bubble formation starting around $15 \mathrm{~mJ} / \mathrm{cm}^{2}$, using a theoretical bubble formation threshold of $\rho_{\mathrm{bf}}=1.8 \times 10^{20} \mathrm{~cm}^{-3}$ [75] (Figure 4.5b). This discrepancy is discussed and further analysis is provided in Chapter 6 . 


\section{NS800}
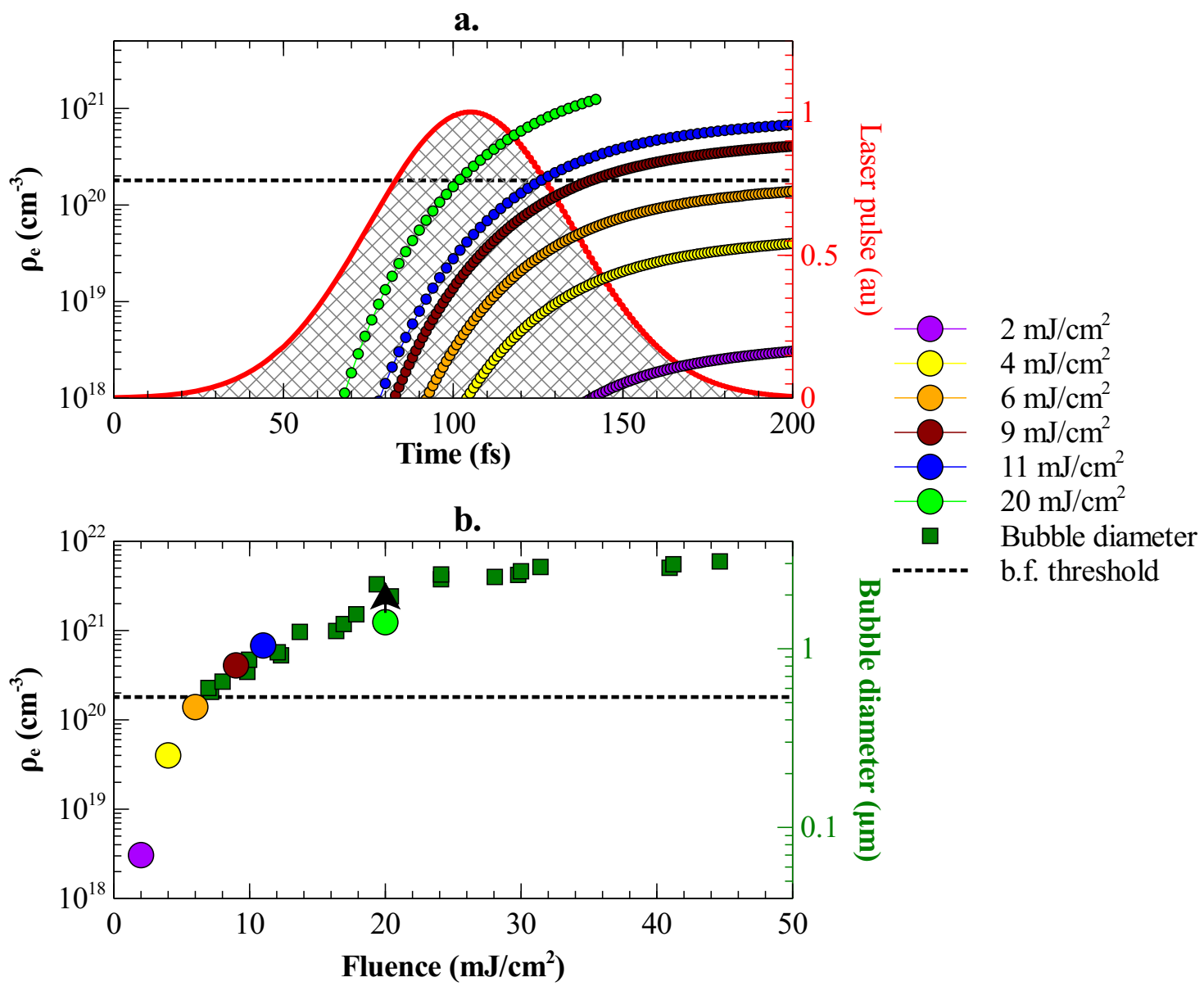

Bubble diameter

Figure 4.4: A comparison of simulation against experimental data published by Lachaine et al. [97] for NS800 gold nanoshell. a. Coloured circles show the temporal electron density predicted by the model in water (at the location of the maximum density). The black dashed line represents the bubble formation threshold for a femtosecond pulse in water, which was set at $\rho_{\text {bf }}=1.8 \times 10^{20} \mathrm{~cm}^{-3}[75]$. The red line represents the temporal profile of the $70 \mathrm{fs}$ laser pulse. b. The blue squares show the experimentally measured bubble diameters at various fluences and the coloured circles show the maximum electron density calculated by the model during $70 \mathrm{fs}$ pulse duration for various fluences. The black arrows indicates models where calculation did not reach the end of the $70 \mathrm{fs}$ pulse. Due to the increased complexity of the model and the size of the nanoshell, the simulation with a $20 \mathrm{~mJ} / \mathrm{cm}^{2}$ fluence did not complete within 70 fs pulse duration. 


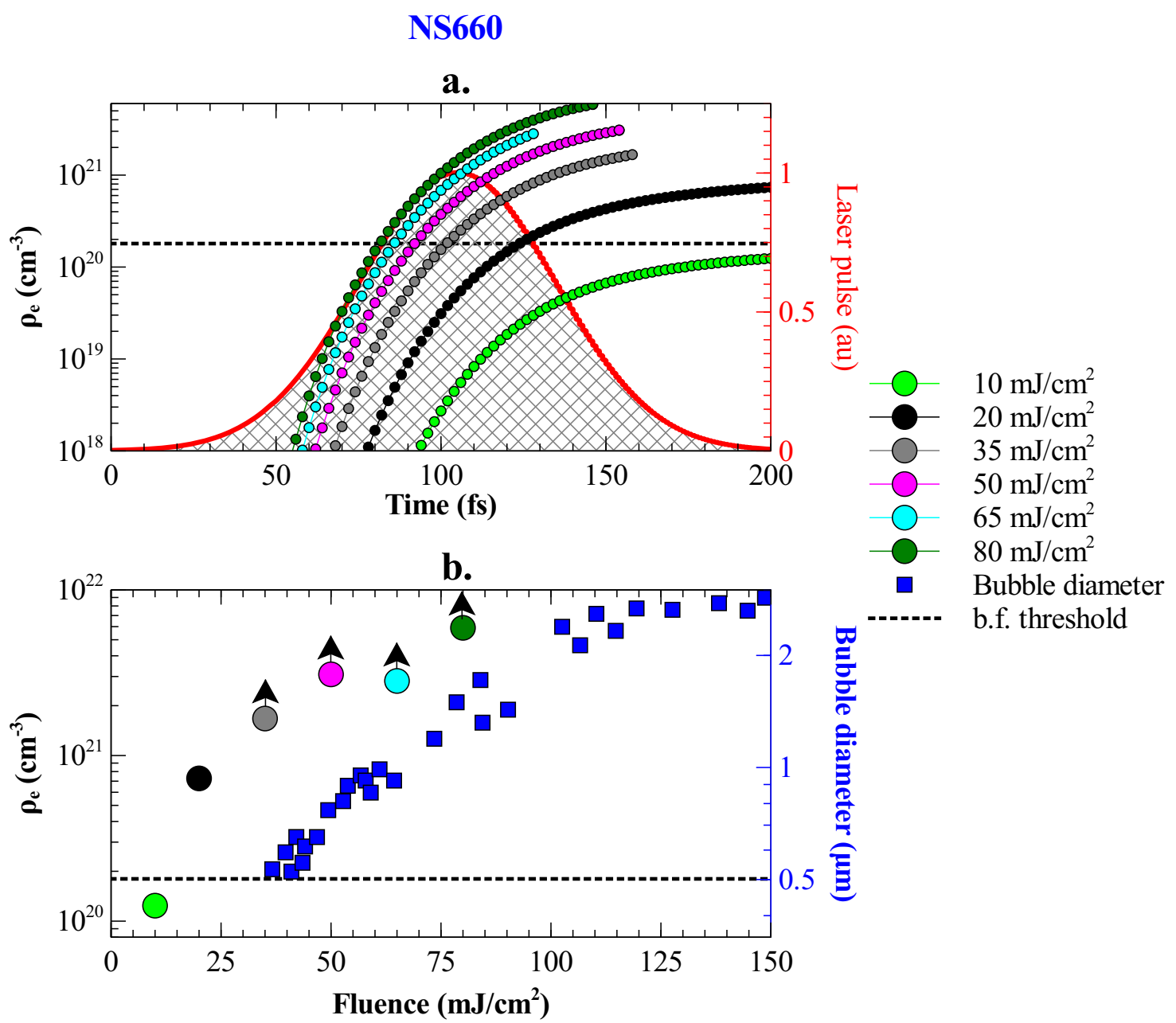

Figure 4.5: A comparison of simulation against experimental data published by Lachaine et al. 97] for NS660 gold nanoshell. a. Coloured circles show the temporal electron density predicted by the model in water (at the location of the maximum density). The black dashed line represents the bubble formation threshold for a femtosecond pulse in water, which was set at $\rho_{\mathrm{e}}=1.8 \times 10^{20} \mathrm{~cm}^{-3}[75$ ]. The red line represents the temporal profile of the $70 \mathrm{fs}$ laser pulse. b. The blue squares show the experimentally measured bubble diameters at various fluences and the coloured circles show the maximum electron density calculated by the model during $70 \mathrm{fs}$ pulse duration for various fluences. The black arrows indicates models where calculation did not reach the end of the 70 fs pulse. 


\subsection{The role of the environmental on the optical prop- erties of gold nanorods}

As described in Section 3.2, the optical response of three individual silica coated gold nanorods ( $\mathrm{S}_{-} \mathrm{GNR}_{1}, \mathrm{~S}_{-} \mathrm{GNR}_{2}$, and $\mathrm{S}-\mathrm{GNR}_{3}$ ) on a TEM grid was measured and modelled (Figure 4.6). The modelled optical response of the GNRs was very close to the measured optical extinction spectrum (Figure 4.7a). Initially the dimensions of the GNRs in the model were estimated from TEM, but due to the strong dependence of $\lambda_{\mathrm{R}}$ to the GNR aspect ratio, a refined fitting to the measured curve was performed by slightly resizing the rod length/width (by less than $0.5 \mathrm{~nm}$ ) and consequently its aspect ratio (Table 3.1). This refinement fell within the precision of the TEM and optical measurements. The good reproduction of the SPR spectral position and shape for these silica coated nanoparticles validated the use of the dielectric function for gold reported by Johnson and Christy [115] for $\tilde{\varepsilon}_{\mathrm{B}}$ in equation 3.1. An approximately $4 \mathrm{~nm}$ red or blue shift of $\lambda_{\mathrm{R}}$ is obtained if the gold dielectric functions reported by Blanchard et al. [159] or Palik [160] are used compared to using the Johnson and Christy [115] dielectric data, respectively [88, 110].

The model was unable to reproduce the measured spectra for the bare GNRs $\left(\mathrm{GNR}_{4}\right.$, $\mathrm{GNR}_{5}$, and $\mathrm{GNR}_{6}$ ) using dimensions that were within the TEM measurement uncertainty. The spectra of the bare gold nanorods were then computed using the smallest and largest aspect ratios within the uncertainty limits from the TEM measured dimensions. These spectra strongly deviated from the experimental ones, especially with respect to the peak wavelength $\lambda_{\mathrm{R}}$, as shown in Figure 4.7b. Even when using the largest aspect ratio within the uncertainty limits of the TEM measurements, the computed $\lambda_{\mathrm{R}}$ was blue-shifted by $55-80 \mathrm{~nm}$. Assuming that this discrepancy is due to under-estimation of the rod aspect ratio in the TEM measurements, fitting of the experimental spectra using the rod dimensions as parameters required an increase of the aspect ratio between $25 \%$ and $50 \%$ (depending on the GNR), which is incompatible with the precision of the TEM measurements.

For nonspherical particles, such as nanorods, both the object shape and the environment determine $\lambda_{\mathrm{R}}$, and a proper reproduction of the measured spectra requires more realistic modeling of the actual environment. Although previous studies have shown that GNRs have octagonal cross-section [161, 162], in the numerical model the GNRs were 
Silica-coated GNR

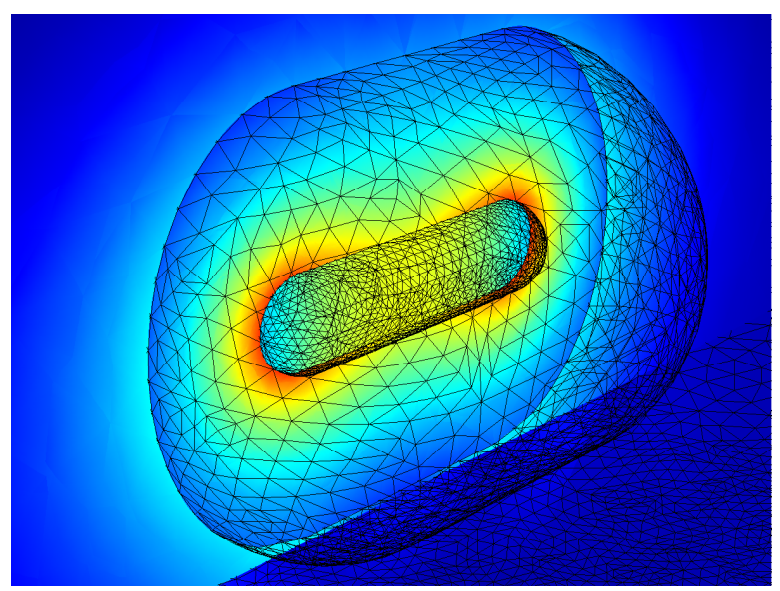

Bare GNR

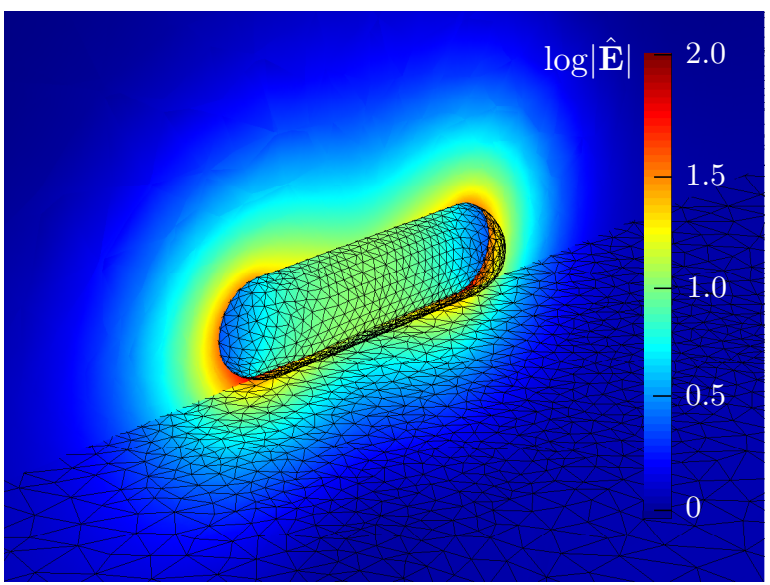

Figure 4.6: Plots of the relative electric field, $\hat{\mathbf{E}}=|\mathbf{E}| /\left|\mathbf{E}_{\text {inc }}\right|$, where $|\mathbf{E}|=\sqrt{\mathbf{E} \cdot \mathbf{E}^{*}}$ is the amplitude of the total electric field and $\mathbf{E}_{\mathrm{inc}}$ is the amplitude of the incident field. Superimposed on this is the surface discretization of the gold, silica coating, and substrate regions. $\hat{\mathbf{E}}$ is shown on a colour log-scale. Therefore red represents a field amplification of $100 \times$, cyan represents an amplification of $10 \times$ and dark blue represents a field amplitude equal to the incident field. The GNR shown was approximately $33 \mathrm{~nm}$ long. Reprinted with permission from [26]. Copyright 2016 American Chemical Society.

assumed to have circular cross sections for simplicity. The level of agreement between the model and the measurement for silica-coated GNRs indicates that this approximation plays a minor role in the modeling. Additionally, surfactant molecules or solvent left during spin-coating of the colloidal solution or a water layer due to humidity may have influenced the measured optical spectra. To provide an insight into the impact of variations in the environment on the extinction spectra of bare and silica-coated GNRs, calculations were performed for different environments for a GNR with gold dimensions corresponding to those of $\mathrm{S}_{-\mathrm{GNR}_{1}}$ (Table 3.1). The results are shown in Figure 4.8. As expected, the computed $\lambda_{\mathrm{R}}$ of the bare GNR shows a large dependence on its surrounding, red-shifting by about $130 \mathrm{~nm}$ when changing from a homogeneous air environment $\left(n_{\text {sur }}=1.00\right)$ to a homogeneous water environment $\left(n_{\text {sur }}=1.33\right)$. The same nanorod deposited on a silica substrate in air shows an intermediate $\lambda_{\mathrm{R}}$, demonstrating that in this inhomogeneous configuration (containing both silica and air) the effective refractive index of a homogeneous surrounding medium is lower than that for water. Conversely, $\lambda_{\mathrm{R}}$ of 

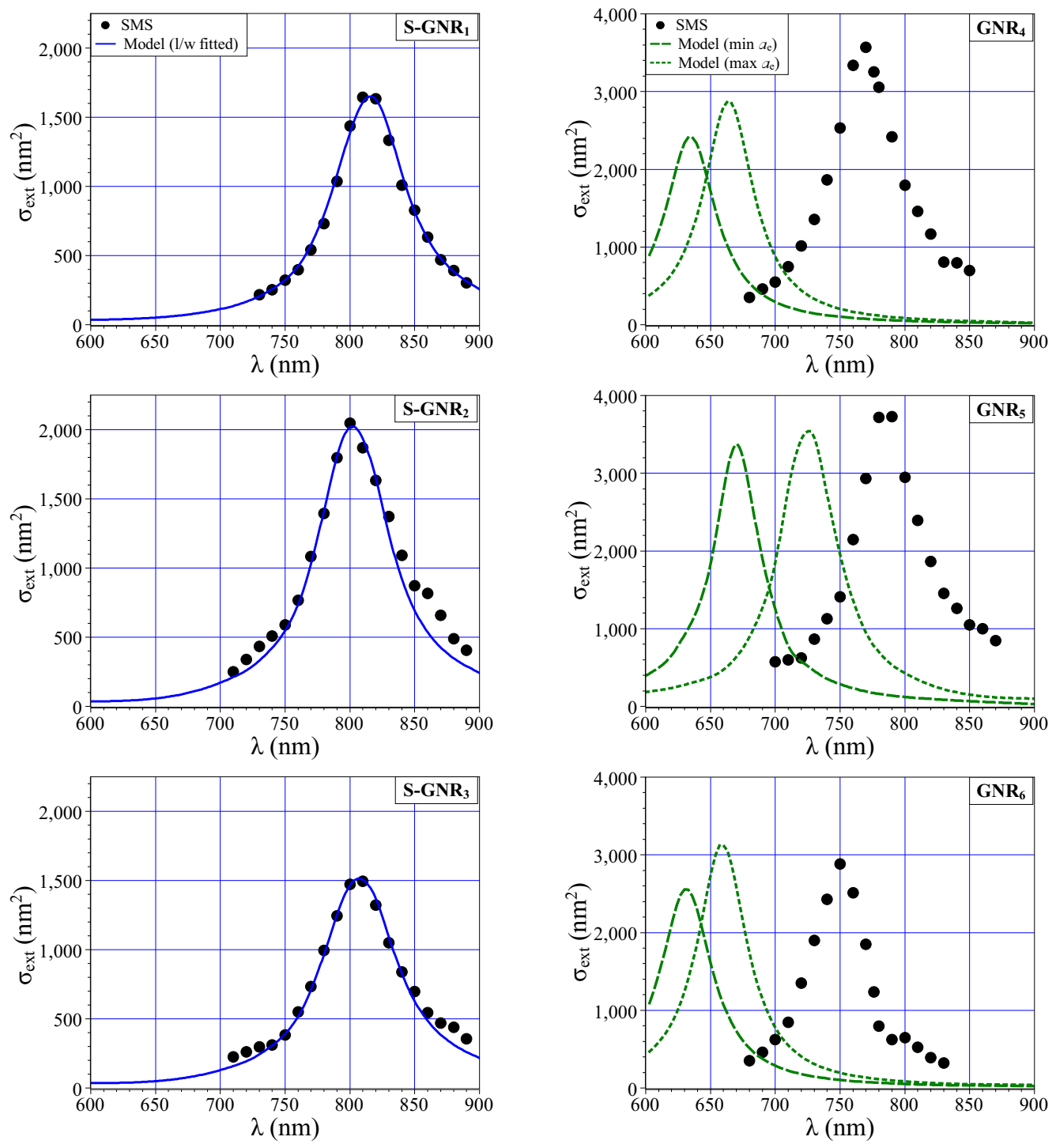

(a) Silica-coated rods.

(b) Bare rods.

Figure 4.7: Simulated (solid/dashed/dotted lines) and measured (dots) absolute extinction cross-sections of (a) silica-coated GNR; and (b) bare GNRs, all on a silica substrate in air. The simulated spectra shown in the silica-coated GNRs are from a best-fit for the GNR and silica dimensions, as described in the Section 3.2. The fitted dimensions (Table 3.1) are within the TEM measurement uncertainty in all three cases. For the bare GNRs, since the GNR aspect ratio affects the extinction peak position, the smallest and largest aspect ratios that fit within the uncertainty of the dimensions from the TEM images $( \pm 0.5 \mathrm{~nm})$ were used to produce the two simulated extinction spectra. Reprinted with permission from [26]. Copyright 2016 American Chemical Society. 
the silica-coated GNR exhibits a much smaller environment dependence, only red-shifting by about $20 \mathrm{~nm}$ from a homogeneous air to a homogeneous water environment. This reduced sensitivity simply reflects the fact that the plasmonic response of a nanoparticle is sensitive to its environment on the spatial range over which field enhancement takes place, typically of the order of the rod diameter for the investigated rods (Figure 4.6 and 4.9a and of the order of the particle radius for a sphere [88]. Finally, placing a $20 \mathrm{~nm}$ water layer around the bare GNR while on the substrate, in air, results in $\lambda_{\mathrm{R}}$ red-shifting by approximately $100 \mathrm{~nm}$ (compared to a red-shift of $10 \mathrm{~nm}$ for the silica-coated rod), yielding a value close to what was measured.

As the SMS technique provides a quantitative measurement of light extinction, further information can be obtained by analyzing the amplitude of the measured extinction spectra, $\sigma_{\text {ext }}\left(\lambda_{\mathrm{R}}\right)$. There are large variations, of up to $800 \mathrm{~nm}^{2}$ ( $22 \%$ of the mean), in $\sigma_{\text {ext }}\left(\lambda_{\mathrm{R}}\right)$ in the SMS measurements between the three bare rods shown in Figure 4.7b. This may be partly accounted for by differences in the GNR volumes, but may also be due to environment fluctuations. Figure 4.8 provides some insight into the effect of the environment on $\sigma_{\text {ext }}\left(\lambda_{\mathrm{R}}\right)$. The presence of water around the bare GNR (both as an infinite homogeneous medium and as a $20 \mathrm{~nm}$ layer on a substrate) increases $\sigma_{\text {ext }}\left(\lambda_{\mathrm{R}}\right)$ by about $600 \mathrm{~nm}^{2}$, partly accounting for the observed variations.

A parametric analysis was performed to systematically study the effect of possible contamination on the optical response of bare and coated GNRs. This was done by simulating $\mathrm{S}_{-} \mathrm{GNR}_{1}$ with and without its silica shell. In the first study the GNR was surrounded by a drop of water of varying thickness and deposited on a silica substrate layer (see Figure 3.4). Figure 4.9a shows that for silica-coated rods the water droplet on top of the GNR and around its ends produced a weak $\lambda_{\mathrm{R}}$ red-shift of up to $13 \mathrm{~nm}$, while for bare rods the water layer produced a strong $\lambda_{\mathrm{R}}$ red-shift up to $100 \mathrm{~nm}$ as the drop thickness increases, reaching a plateau after $20 \mathrm{~nm}$ in thickness (about $90 \%$ of the shift is observed for a thickness of $10 \mathrm{~nm}$, which is of the order of the rod diameter). The effect of the droplet on the spectrum's fwhm was also more pronounced for the bare GNR compared to the silica-coated one. This is a consequence of the fact that the decrease in fwhm (for all parametric analyses shown in Figure 4.9), given in $\mathrm{eV}$ units, is correlated to the shift in $\lambda_{\mathrm{R}}$. The decrease in fwhm corresponds to a decrease of the imaginary part of the gold dielectric function as $\lambda_{\mathrm{R}}$ is shifted away from interband transitions [46, 88, 104].

Although the results in Figure 4.9a are for a hypothetical GNR, its aspect ratio (based 


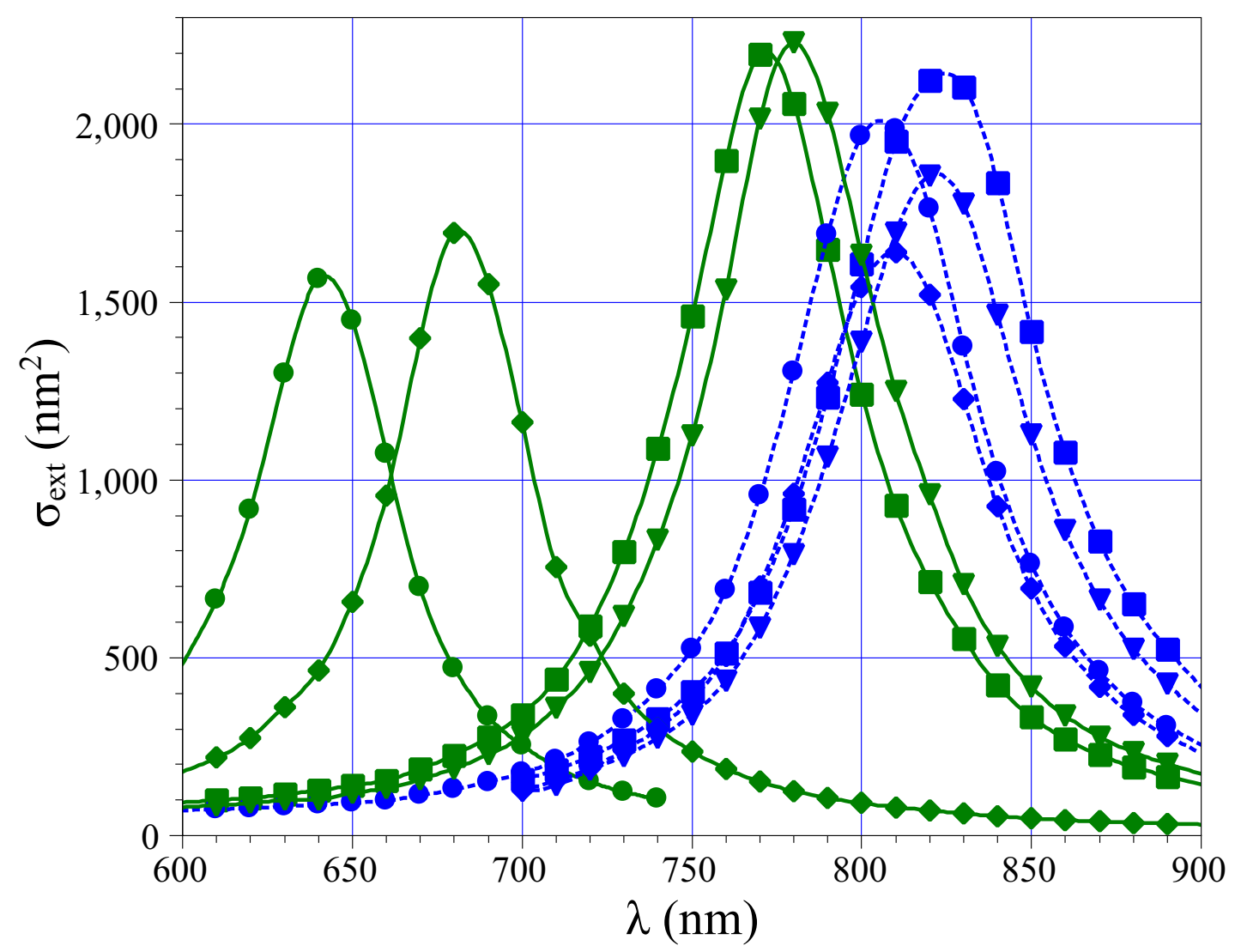

Figure 4.8: Simulated extinction cross-sections of a silica-coated v (blue lines) and a bare GNR (green lines) surrounded by air with no substrate (•), surrounded by water with no substrate $(\mathbf{-})$, deposited on a silica substrate in air $(\bullet)$, and surrounded by a $20 \mathrm{~nm}$ layer of water deposited on a substrate in air ( $\mathbf{v}$; see Figure 3.4). The gold dimensions are those of S-GNR 1 (Table 3.1). Reprinted with permission from [26]. Copyright 2016 American Chemical Society.

on dimensions of the gold part of $\mathrm{S}_{-\mathrm{GNR}_{1}}$ ) is the same as that of $\mathrm{GNR}_{5}$ (see Table 3.1). The SMS measurements for $\mathrm{GNR}_{5}$ (Figure 4.7b) produced $\lambda_{\mathrm{R}}=785 \mathrm{~nm}$, which was redshifted from the simulated spectra by between 60 and $115 \mathrm{~nm}$ (Figure 4.7b). Figure 4.9a shows that a water layer thickness of $s_{\mathrm{w}} \approx 3 \mathrm{~nm}$ on the bare $\mathrm{S}-\mathrm{GNR}_{1}$ results in a red-shift of $\lambda_{\mathrm{R}}$ of $\approx 60 \mathrm{~nm}$, and $s_{\mathrm{w}}>20 \mathrm{~nm}$ results in a red-shift of $\lambda_{\mathrm{R}}$ of $\approx 105 \mathrm{~nm}$. This suggests that a thin layer of water contamination was present during the measurement with a minimum thickness of the order of $3 \mathrm{~nm}$ using the water layer geometry shown above (Figure 3.4). This value has however to be considered as a very rough estimate since 

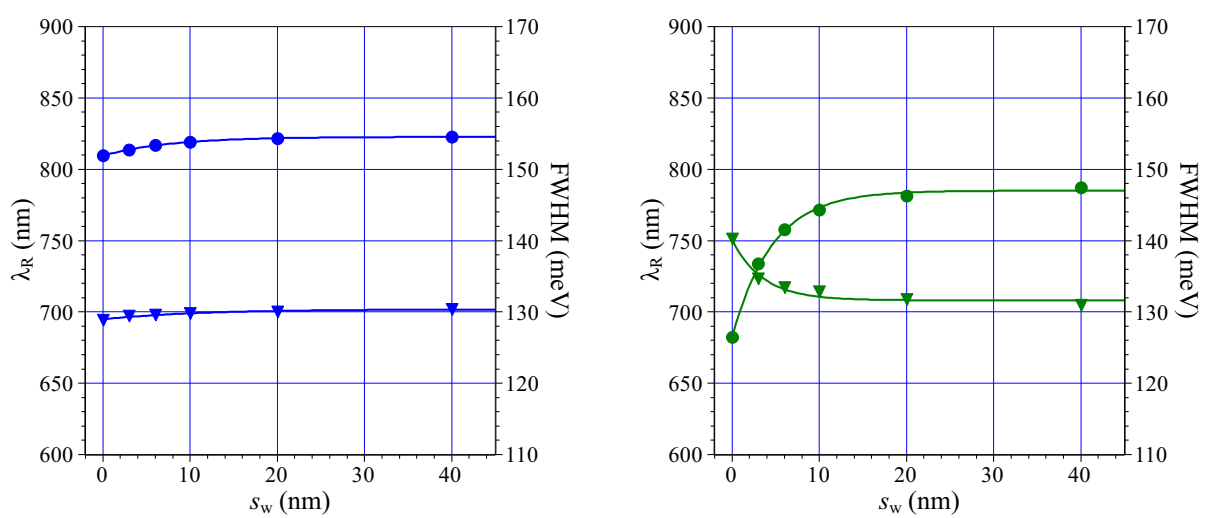

(a) Effect of water droplet size, in air, on a substrate (see Figure 3.4).
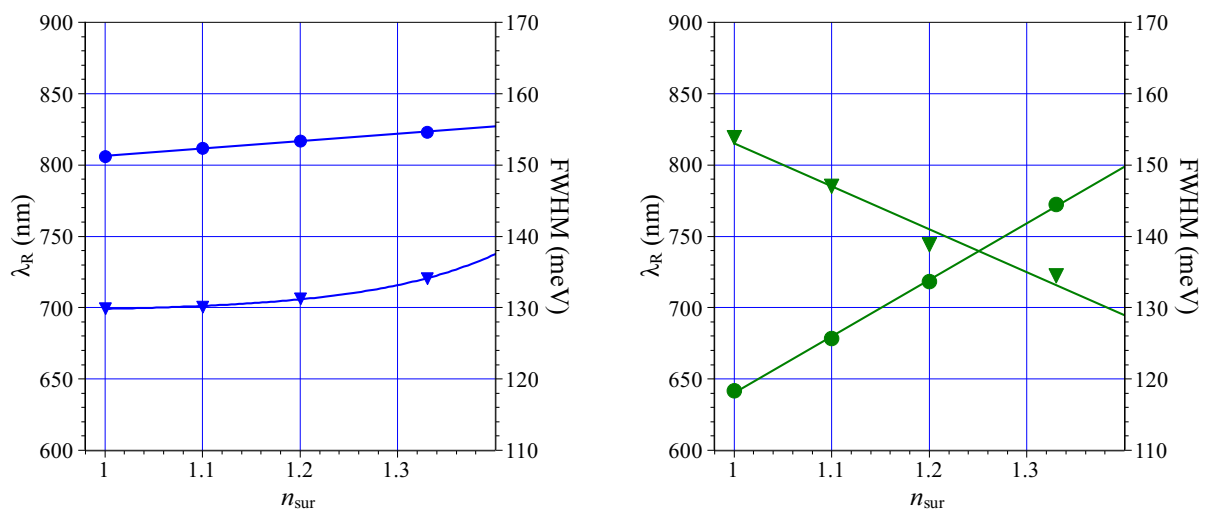

(b) Effect of a homogeneous surrounding medium's refractive index, $n_{\text {sur }}$.
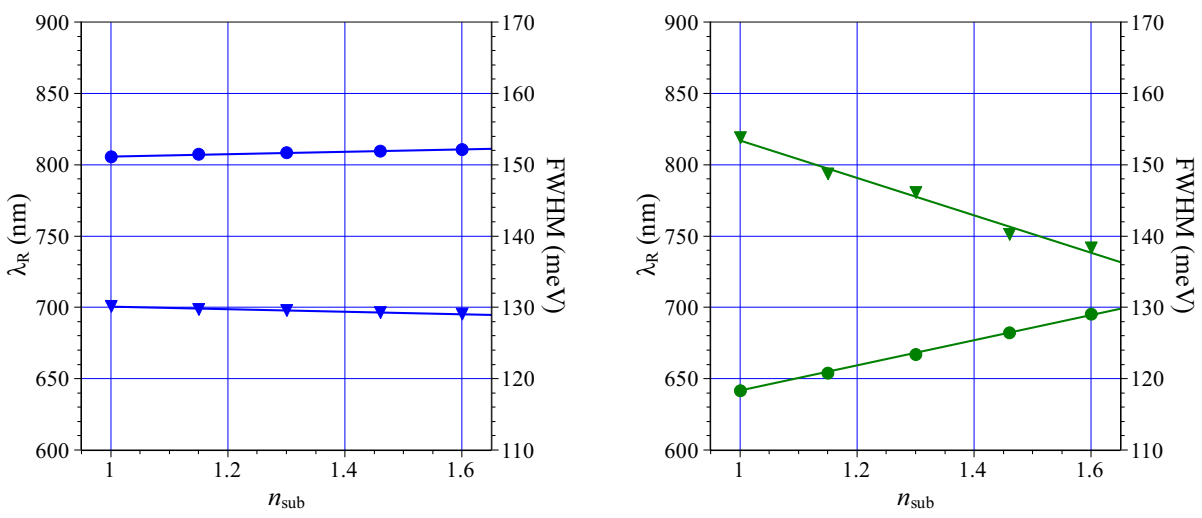

(c) Effect of substrate's refractive index, $n_{\text {sub }}$, in air.

Figure 4.9: A study of how the surroundings of a silica-coated GNR (left graphs) and a bare GNR (right graphs) affect $\lambda_{\mathrm{R}}(\bullet)$ and fwhm $(\boldsymbol{\nabla})$. Reprinted with permission from [26]. Copyright 2016 American Chemical Society. 
the wavelength of the longitudinal SPR of a nanorod is mostly sensitive to the refractive index of its environment around its tips in the rod axis direction, that is, along the substrate plane. Although a nanometer size water layer is expected, the actual thickness experienced by the rod at its tips can thus be larger as the water layer is expected to extend on the substrate plane with a meniscus not accounted for by the model used here (Figure 3.4). A more realistic geometry for the water layer would include different thickness above the rod and along its main axis direction together with a more realistic water meniscus shape. Furthermore, the ligand molecules bound at the nanoparticle surface may also increase the actual dielectric constant experienced by the rod, an effect not included here.

In contrast to the model used here, the presence of the substrate has been frequently accounted for in many single nanoparticle optical studies assuming that the individual nanoparticles are embedded into an effective homogeneous environment with its refractive index, $n_{\text {sur }}$, used as a fitting parameter [88, 108]. Only a few theoretical studies have attempted to include the substrate explicitly in the analysis of single-particle scattering experiments [163, 164]. In the homogeneous environment approximation, $n_{\text {sur }}$, thus incorporates the influence of the substrate and other surrounding materials (air, water, or residual solvent and ligand molecules), with a value that is between the substrate's index and the index of the surroundings. Although it constitutes a crude approximation, masking the complexity of the particle environment in the actual single particle geometry, the effective homogeneous environment approximation has been successfully applied to the investigation of single nanospheres (in contrast to GNRs, the dielectric environment and size effects of nanospheres impact independent parameters for the most part, permitting independent and reliable determination of $n_{\text {sur }}$ and of the particle size from the SMS optical spectra [88, 110]).

In a second parametric study the sensitivity of the spectrum was examined to changes in $n_{\text {sur }}$. As expected, again, the silica-coated rods were much less sensitive to $n_{\text {sur }}$ than the bare rods (Figure 4.9b). $\lambda_{\mathrm{R}}$ changed by approximately $20 \mathrm{~nm}$ for the silica-coated rod compared to a change of approximately $120 \mathrm{~nm}$ for the bare rod (similar effects are observed for the fwhm) between air and water. Using this approach (which accounts for the effect of the substrate by using an effective homogeneous environment) one has to use $n_{\text {sur }} \approx 1.36$ to reproduce the $\lambda_{\mathrm{R}} \approx 785 \mathrm{~nm}$ measured for $\mathrm{GNR}_{5}$. Although this is in-between the refractive index of air and the silica substrate $\left(n_{\text {sub }}=1.46\right)$, it does not 
mean that a homogeneous surrounding with $n_{\text {sur }}=1.36$ accounts for only the effect of the substrate. As shown in Figure $4.7 \mathrm{~b}$ for $\mathrm{GNR}_{5}$ a model that explicitly included the substrate in air was not able to reproduce the $\lambda_{\mathrm{R}} \approx 785 \mathrm{~nm}$ SMS measurement for $\mathrm{GNR}_{5}$. This indicates that environmental contamination (such as a thin water layer or residual surfactants) must have affected the SMS measurement.

To further analyze the role of the substrate, the influence of the substrate refractive index on the SPR characteristics of a silica-coated and a bare GNR was investigated. As expected, the silica-coated GNR was less influenced by the substrate than the bare GNR (Figure 4.9c). $\quad \lambda_{\mathrm{R}}$ of the silica-coated GNR red-shifted by approximately $5 \mathrm{~nm}$ when changing from no substrate $\left(n_{\text {sub }}=1.00\right)$ to a high index substrate $\left(n_{\text {sub }}=1.60\right)$, whereas it red-shifted by approximately $50 \mathrm{~nm}$ for the bare GNR, from no substrate to a high index substrate.

These quantitative investigations of single particle spectroscopy on bare or coated nanoparticles stress the importance of controlling the local nanoparticle environment. The lower sensitivity of silica-coated GNRs directly reflects the fact that nanoparticles experience only their close environment on a distance of the order of the spatial extent of the local field around the particle at $\lambda_{\mathrm{R}}$ (Figure 4.6). This field is of the order of the radius for a sphere [44, 165], or of the width of a GNR (the studied GNRs had widths between approximately 8 and $11 \mathrm{~nm}$ ) [166]. The three GNRs in this study were coated with silica shells of thickness approximately between 8 and $16 \mathrm{~nm}$ resulting in the environment having little impact on the GNR's local field.

\subsection{The role of gold nanoparticle morphology and cou- pling in optical breakdown during picosecond pulse exposures}

The interaction of a 6 ps laser pulse with the gold nanostructures in an aqueous environment listed in Table 4.1 was modelled. The spatial distribution of the electric field enhancement, the free electron density in medium and the lattice temperature of gold for the nanostructures are shown in Figure 4.10. For uncoupled nanoparticles (Figure $4.10 \mathrm{a}, \mathrm{d})$ the maximum electric field enhancement, $E_{\mathrm{e}, \max }=\max |\mathbf{E}| / E_{0}$, is located 


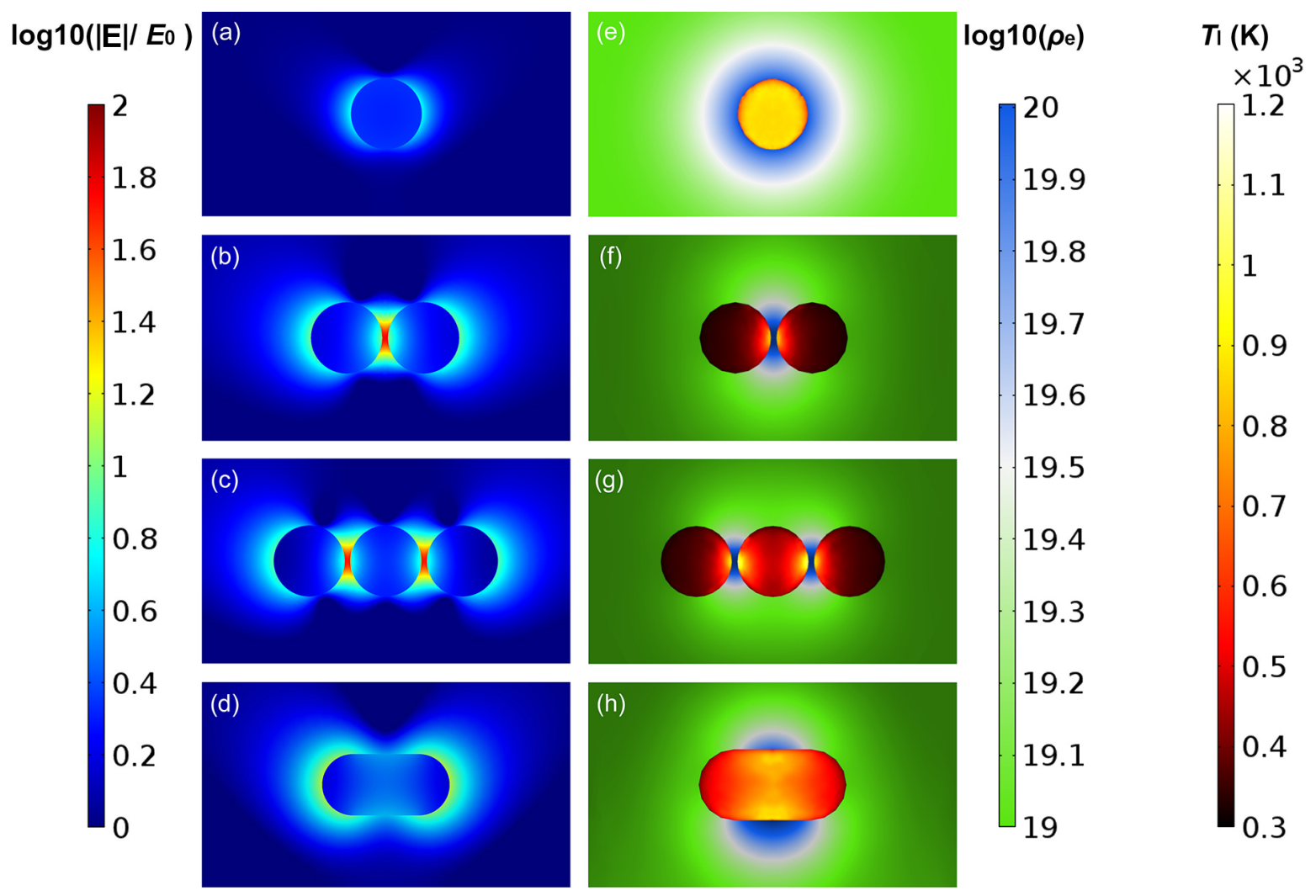

Figure 4.10: (a)-(d) plots of the relative electric field enhancement, $\log _{10}\left(|\mathbf{E}| / E_{0}\right)$, of $25 \mathrm{~nm}$ nanospheres and nanorod, where $|\mathbf{E}|$ is the amplitude of the calculated electric field and $E_{0}$ is the amplitude of the incident electric field, polarized along long axis of the nanostructure with propagation from the bottom of the page towards the top. (e)-(h) the $\log$-scale of free electron density plasma, $\log _{10}\left(\rho_{\mathrm{e}}\right)\left(\mathrm{cm}^{-3}\right), 4 \mathrm{ps}$ delayed after the temporal peak of incident laser pulse intensity and the lattice temperature, $T_{1}(\mathrm{~K})$, of the nanoparticle sampled at the end of the pulse duration. All plots are produced for nanoparticles exposed at resonance wavelength (see Figure 4.12).

at the poles of the nanoparticle while for dimers and trimers (Figure $4.10 \mathrm{~b}, \mathrm{c}$ ) the maximum field enhancement is in the region in-between the nanoparticles. The spectra of the maximum field enhancement of these nanostructures are shown in Figure 4.11 .

The Figure 4.12 a summarize of the maximum near-field enhancement for all the nanostructures at the resonance wavelength and at $\lambda=532 \mathrm{~nm}$ (the second harmonic wavelength of popular solid-state Nd:YAG lasers). The resonance peak position of the nanosphere monomers is located around $\lambda=550 \mathrm{~nm}$ (Figure 4.11a) and is close to $532 \mathrm{~nm} . E_{\mathrm{e}, \max }$ at resonance for monomers increases with increasing radius, and has a 
$2.7 \%$ increase from $5 \mathrm{~nm}$ to $25 \mathrm{~nm}$ nanospheres. The change from a nanosphere monomer to a dimer and trimer induce a red shift in the plasmon resonance peak and leads to an increase in $E_{\mathrm{e}, \max }$. Hovewer, this increase drops from $8.5 \%$ for $5 \mathrm{~nm}$ diameter nanospheres to $0.9 \%$ for $25 \mathrm{~nm}$ diameter nanospheres. The highest changes in the maximum field enhancement at resonance were found between the $25 \mathrm{~nm}$ nanosphere monomer and its assemblies. The maximum field enhancement of the $25 \mathrm{~nm}$ trimer (s25t@640) was 9 times higher than of the $25 \mathrm{~nm}$ monomer at resonance wavelength and 11.5 times higher than of the $25 \mathrm{~nm}$ monomer (s25m@532) at $532 \mathrm{~nm}$ wavelength. A plasmon resonance shift, $\lambda_{\text {shift }}=80 \mathrm{~nm}$, can be seen when the assembly builds up from a $25 \mathrm{~nm}$ monomer (s25m) to a $25 \mathrm{~nm}$ trimer (s25t). For dimer and trimer nanospheres, $E_{\mathrm{e}, \max }$ increases with the size of a nanosphere and has a small dependence on the number of the nanoparticles. For nanorods, $E_{\mathrm{e}, \max }$ decreases as the radius of the nanorod increases. The aspect ratio of the nanorods does not affect $E_{\mathrm{e}, \max }$, except for the $5 \mathrm{~nm}$ nanorod.

In the case of the nanosphere monomer, there is an almost a uniform distribution of temperature across the nanosphere volume (Figure 4.10 ), while for nanosphere assemblies and the gold nanorod, the temperature profile reveals hot and cold zones across the particle's volume (Figure 4.10-h).

Figure $4.12 \mathrm{~b}$ shows the maximum gold lattice temperature, $T_{1, \max }$, at the time $4 \mathrm{ps}$ after the temporal peak of the laser pulse. For all nanostructures, the maximum lattice temperature was below the $1337 \mathrm{~K}$, the bulk melting point of gold (Figure $4.12 \mathrm{~b}$ ). This melting point of gold is valid for nanoparticles with radius of more than $5 \mathrm{~nm}$ [68]. The lowest heating was produced using a gold nanosphere trimer, s25t@532, exposed at the off-resonance wavelength of $532 \mathrm{~nm}$.

Figure 4.10 $\mathrm{k}$-h shows that the location of the maximum free electron plasma density surrounding the nanostructures is adjacent to the location of the gold lattice hot temperature spots, which also corresponds to the location of the maximum electric field enhancement inside of the particles (Figure 4.11 b-d and 4.11 $\mathrm{f}-\mathrm{h}$ ). This means that during a 6 ps pulse interaction gold nanoparticles strongly absorb light (this is called the absorption regime) and photo-thermal emission dominates the seed electron production.

Figures 4.12 -d shows the volume $\left(V_{\mathrm{np}}\right)$ and the absorption cross-section $\left(\sigma_{\mathrm{abs}}\right)$ of the nanoparticles and their assemblies. The absorption cross-section for uncoupled nanoparticles and assemblies increases with the size of the nanoparticle, and also increases when 
on-resonance excitation is used. Figure 4.12 shows $F_{\text {th }}$, the laser fluence needed to reach

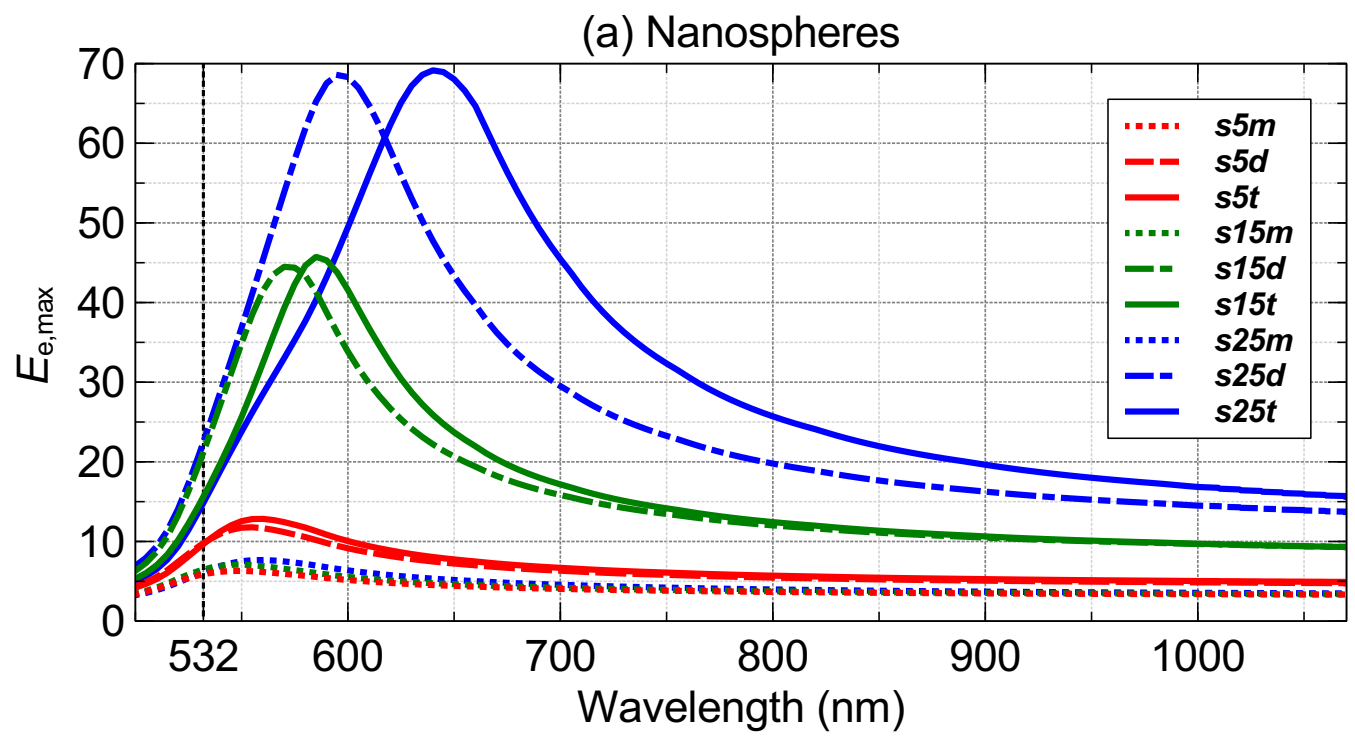

(b) Nanorods

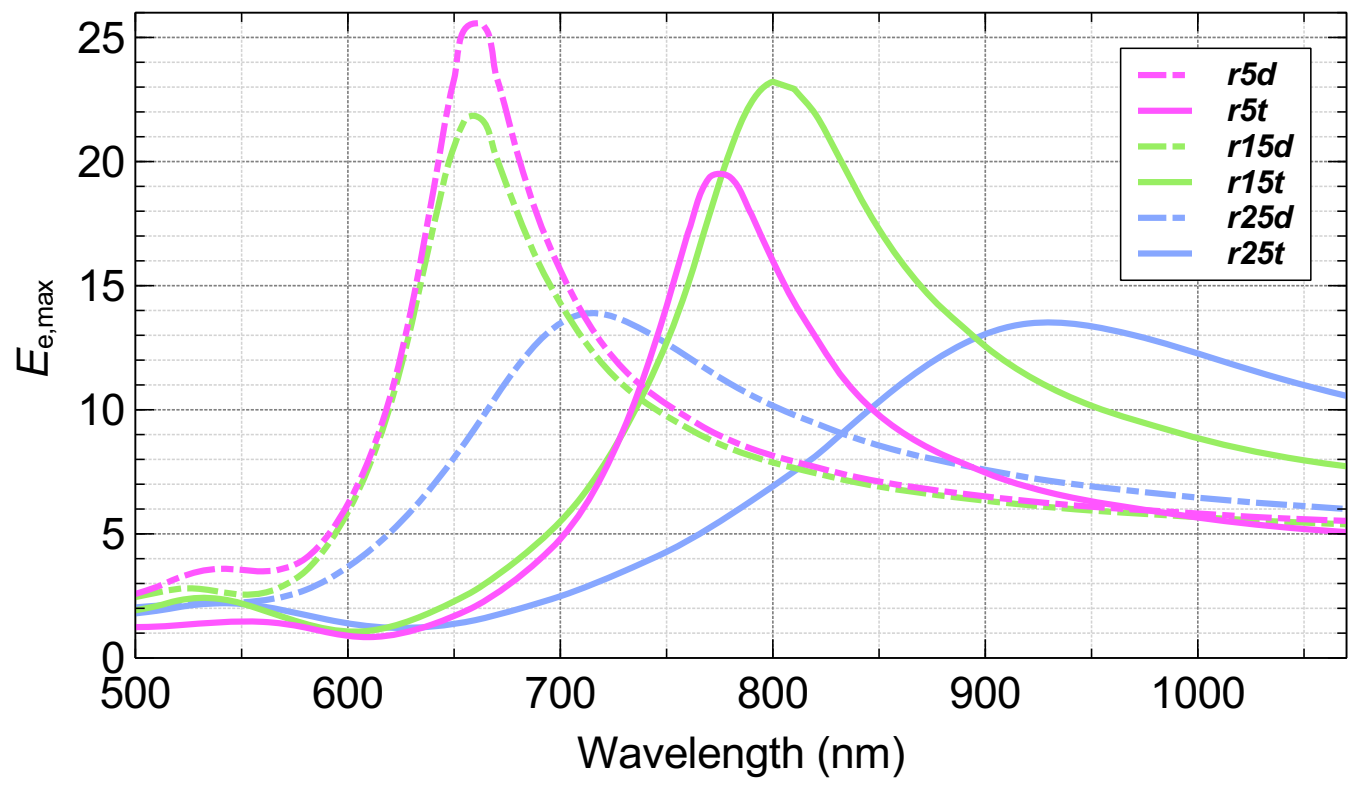

Figure 4.11: Maximum near-field enhancement, $E_{\mathrm{e}, \max }$, located at the hot zones of the assemblies or poles of the monomers (see Figures $4.10 \mathrm{a}-\mathrm{d}$ ). The dimensions of the particles are given in Table 4.1 . 


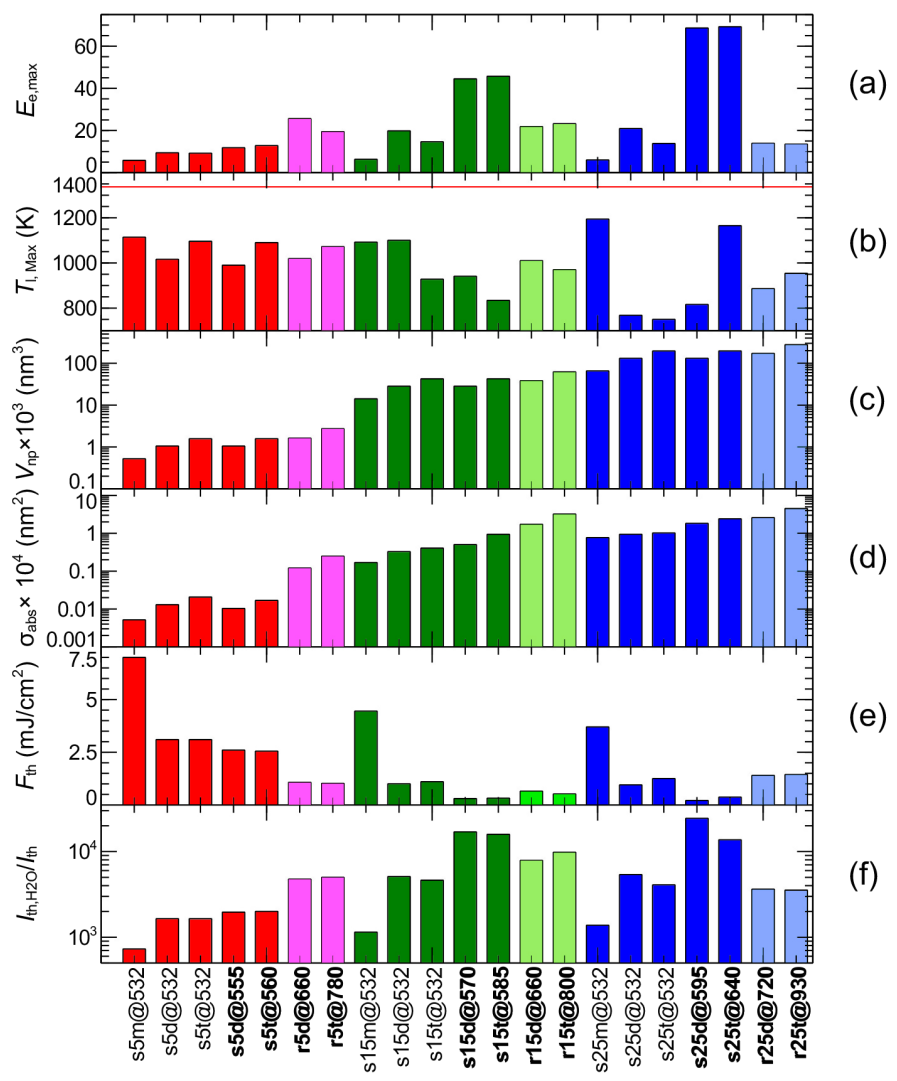

Figure 4.12: Nanoparticles of different morphology used in the model and corresponding parameters obtained to reach optical breakdown. The first letter of the nanoparticle's label (e.g. s5m@532)," s" or "r", stands for nanosphere and nanorod, respectively. The numbers 5, 15 and 25, corresponds to radius of the nanoparticle in nanometers. "m", "d", "t" stands for monomer, dimer and trimer, respectively. The last number of the nanoparticle's label corresponds to the wavelength, $\lambda(\mathrm{nm})$, used in the simulations. Bold labels corresponds to nanostructure in-resonance. Bar plot (a), $E_{\mathrm{e}, \max }$, provides the data of the maximum electric field enhancement that is located in the nanoparticle's hot zone (see Figures $4.10 \mathrm{a}-\mathrm{d}$ ). (b) shows the maximum lattice temperature, $T_{1, \mathrm{Max}}$, reached at the end of the laser pulse duration, measured in the hot zone of the particle (Figures 4.10 $-\mathrm{h}$ ) (the red line marks melt-

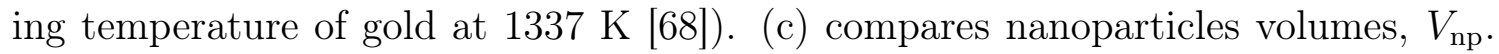
(d) corresponds to absorption cross-section, $\sigma_{\text {abs }}$, of the nanoparticles and their assemblies. (e) compares the laser fluence, $F_{\mathrm{th}}$, needed to reach critical density of the free electrons for bubble formation, $\rho_{\mathrm{cr}}=10^{20} \mathrm{~cm}^{-3}$ [27, 55], in the vicinity of nanoparticle. (f) provides the ratio of optical breakdown threshold intensity for a pure water for 3 ps pulses at $580 \mathrm{~nm}, I_{\mathrm{th}, \mathrm{H}_{2} \mathrm{O}}=8.5 \times 10^{11}\left(\mathrm{~W} / \mathrm{cm}^{2}\right)$ [167] and an aqueous environment containing gold nanoparticles, $I_{\text {th }}$ (this study). 
a critical density of the free electrons, $\rho_{\mathrm{cr}}=10^{20} \mathrm{~cm}^{-3}[55]$ in the vicinity of each nanostructure. This corresponds to the free electron density for bubble formation, which was experimentally observed by Vogel et al. [27]. Although most studies have used a value of $\rho_{\mathrm{cr}}=10^{21} \mathrm{~cm}^{-3}$ as a critical plasma density required for the optical breakdown [27], in this study $\rho_{\mathrm{cr}}=10^{20} \mathrm{~cm}^{-3}$ was used in accordance to recent findings by [74, 75], that revealed a lower threshold than previously assumed. By comparison of Figures 4.12k, $4.12 \mathrm{~d}$ and 4.12 , one can observe some correlation between $V_{\mathrm{np}}, \sigma_{\mathrm{abs}}$ and $F_{\mathrm{th}}$, where a higher fluence threshold is needed for smaller absorption cross-section and volume of the nanoparticle. Figure 4.12f, provides the ratio of the optical breakdown threshold intensity for a pure water (for $3 \mathrm{ps}$ pulses at $580 \mathrm{~nm} I_{\mathrm{th}, \mathrm{H}_{2} \mathrm{O}}=8.5 \times 10^{11} \mathrm{~W} / \mathrm{cm}^{2}$ [167]) to the

Table 4.1: Gold nanoparticle types and dimensions used in the model. In particle's type the letters "s", "r", "m", "d" and "t" stands for sphere, rod, monomer, dimer and trimer, respectively. The number in the particle's type defines a radius of the nanosphere and nanorod in nanometers. The gold nanorods were modelled having an overall size of the corresponding nanosphere assembly with matching aspect ratios. For nanosphere assemblies the inter-particle distance, edge to edge, was kept at a constant value of $4 \mathrm{~nm}$. Nanoparticles volume, $V_{\mathrm{np}}$, for gold nanosphere assemblies is given as a sum of individual nanoparticle volumes.

\begin{tabular}{|c|c|c|c|}
\hline Nanostructure & Aspect ratio & Overall size $\mathbf{( n m})$ & $\mathbf{V}_{\mathrm{np}} \times \mathbf{1 0}^{\mathbf{3}} \mathbf{n m}^{\mathbf{3}}$ \\
\hline \multicolumn{4}{|c|}{ Nanospheres } \\
\hline s5m & $1: 1$ & $10 \times 10$ & 0.52 \\
s15m & $1: 1$ & $30 \times 30$ & 14.14 \\
s25m & $1: 1$ & $50 \times 50$ & 65.45 \\
\hline s5d & $2.4: 1$ & $24 \times 10$ & 1.05 \\
s15d & $2.13: 1$ & $64 \times 30$ & 28.27 \\
s25d & $2.08: 1$ & $104 \times 50$ & 130.90 \\
\hline s5t & $3.8: 1$ & $38 \times 10$ & 1.57 \\
s15t & $3.26: 1$ & $98 \times 30$ & 42.41 \\
s25t & $3.16: 1$ & $158 \times 50$ & 196.35 \\
\hline \multicolumn{4}{|c|}{ Nanorods } \\
\hline r5d & $2.4: 1$ & $24 \times 10$ & 1.62 \\
r15d & $2.13: 1$ & $64 \times 30$ & 38.17 \\
r25d & $2.08: 1$ & $104 \times 50$ & 171.48 \\
\hline r5t & $3.8: 1$ & $38 \times 10$ & 62.20 \\
r15t & $3.26: 1$ & $98 \times 30$ & 277.51 \\
r25t & $3.16: 1$ & $158 \times 50$ &
\end{tabular}




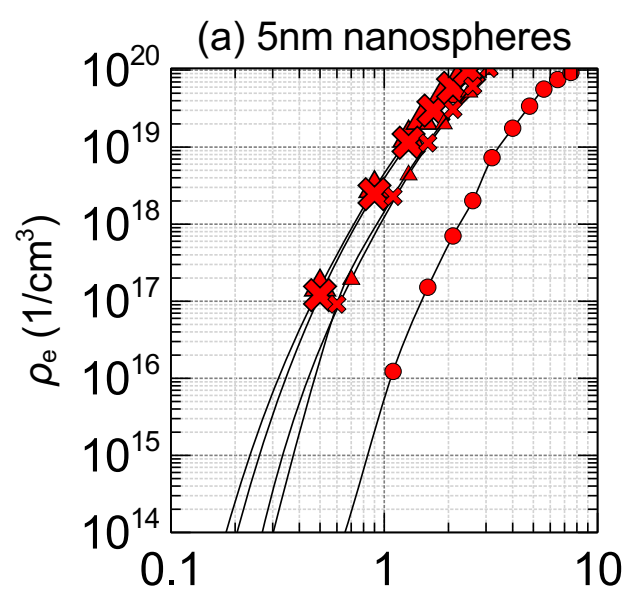

(c) $25 \mathrm{~nm}$ nanospheres

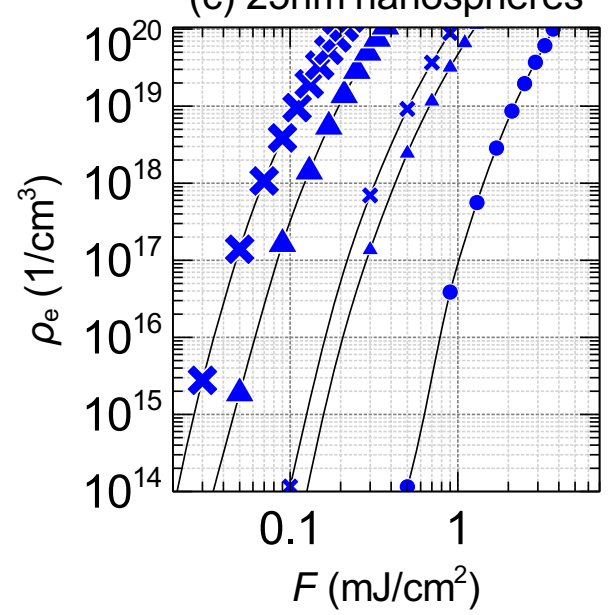

(b) $15 \mathrm{~nm}$ nanospheres

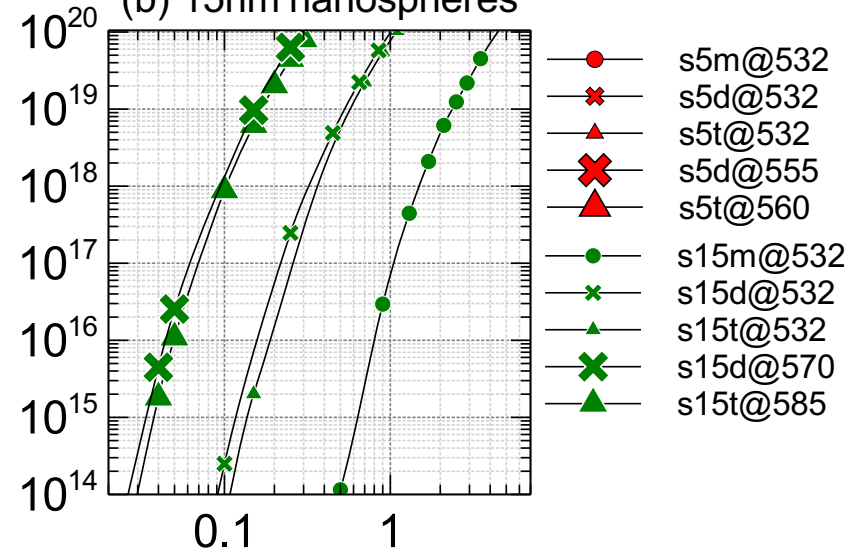

(d) $5 / 15 / 25 \mathrm{~nm}$ nanorods

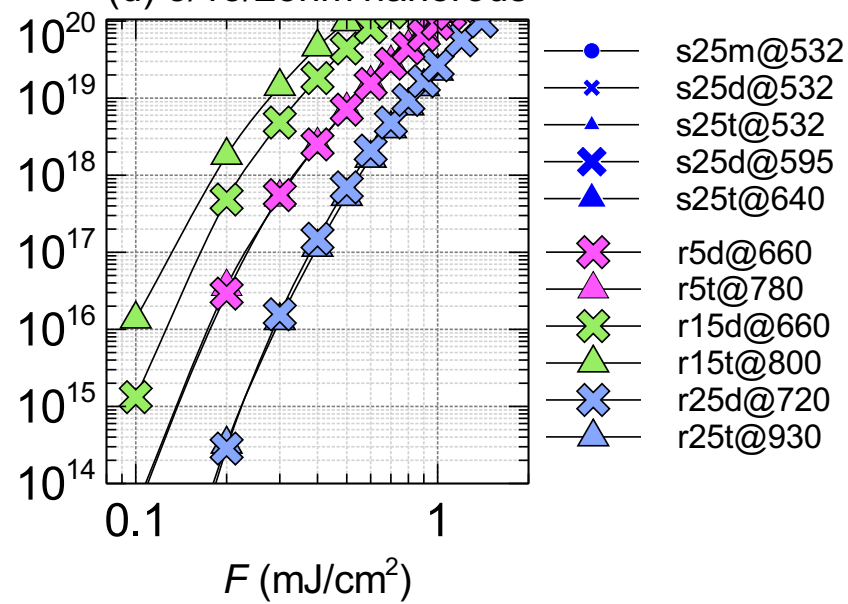

Figure 4.13: Calculated free electron densities for different nanoparticle morphology and wavelengths exposed to 6 ps laser pulse at different fluencies, $F$. The legend provides information on nanoparticle type, where "s"-sphere and "r"-rod; incident wavelength (e.g. "@532" - correspond to $532 \mathrm{~nm}$ ) of the laser pulse and aspect ratio of the nanosphere assembly and nanorod, where "m"-monomer (o symbol), "d"-dimer ( $\times$ symbol), "t"-trimer $(\triangle$ symbol); the numbers 5 (red), 15 (green), 25 (blue) and 5 (light red), 15 (light green), 25 (light blue) corresponds to the nanosphere and nanorod radius in nanometers, respectively.

threshold intensity of water with gold nanoparticles, $I_{\text {th }}$ (this study). This figure shows that the use of gold nanoparticles and their assemblies can decrease the fluence threshold by up to 4 orders of magnitude.

Figure 4.13 shows the impact of the gold nanostructure morphology (nanosphere 
monomer, dimer, trimer and nanorods of different sizes) and laser pulse characteristics (wavelength and fluence) on the plasma density at the location of the highest electric field enhancement. A lower fluence is needed to reach the optical breakdown threshold, for nanosphere dimers and trimers than for monomers, for all three nanoparticle radii. The lowest fluence is needed when the nanosphere dimer and trimer are irradiated at their resonance wavelengths. From Figure $4.13 \mathrm{~d}$ it is evident that gold nanorods of the same radius but with different aspect ratios (mimicking a dimer and a trimer), required similar laser fluences (see Figure 4.12) that are required to achieve the critical plasma density. For example, r5d@660 (nanorod "dimer") needed $F_{\mathrm{th}}=1.07 \mathrm{~mJ} / \mathrm{cm}^{2}$ to reach the critical free electron density, while r5t@780 (nanorod "trimer") needed $F_{\text {th }}=1.02 \mathrm{~mJ} / \mathrm{cm}^{2}$ to reach the same density.

(a)

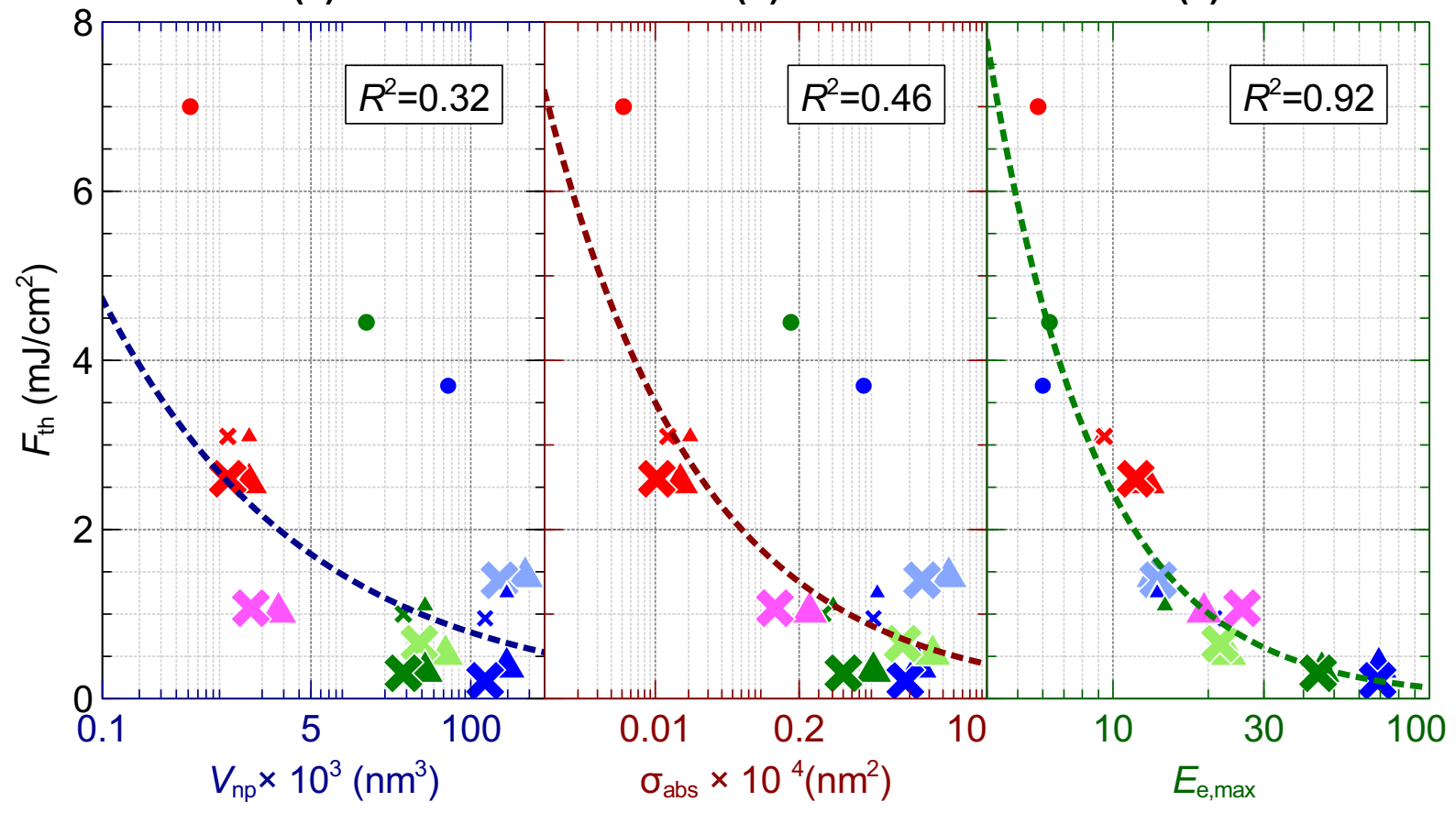

Figure 4.14: The optical breakdown threshold, $F_{\text {th }}$, that is required to reach critical electron density, $\rho_{\text {cr }}=10^{20}\left(\mathrm{~cm}^{-3}\right)$, is plotted against corresponding (a) nanoparticle volume, $V_{\mathrm{np}}$, (b) absorption cross sections, $\sigma_{\mathrm{abs}}$, and (c) maximum near-field enhancement, $E_{\text {e,max }}$. Symbols and colour scheme corresponds to one used in Figure 4.13 at $\rho_{\mathrm{e}}=10^{20}\left(\mathrm{~cm}^{-3}\right)$. 
Figure 4.14 plots the effect of $V_{\mathrm{np}}, \sigma_{\mathrm{abs}}, E_{\mathrm{e}, \max }$, on thresholds $F_{\mathrm{th}}$. The nanoparticles of different morphology but with comparable volumes need different fluencies to reach optical breakdown threshold, which can be seen by comparing r15t@800 against s25m@532; s15t@585, r15d@660 and s15t@532 against each other; and r5d@660 s5t@560 and s5t@532 against each other. On the other hand, an on-resonance exposed s25t@640 and r25d@720 with comparable absorption cross-sections had $F_{\text {th }}=0.37 \mathrm{~mJ} / \mathrm{cm}^{3}$ and $F_{\text {th }}=1.4 \mathrm{~mJ} / \mathrm{cm}^{3}$, respectively. A similar situation can be seen comparing s25d@595 against r15d@660, and s15t@585, s25d@532 and s25t@532 against each other, where the nanoparticles with different morphology but comparable absorption cross-section have different optical breakdown thresholds. A power regression fit of the optical breakdown threshold versus the volume of the nanoparticle yielded, $F_{\mathrm{th}}=2.5969 V_{\mathrm{np}}^{-0.26}$ with $R^{2}=0.32$, and a power regression fit of the optical breakdown threshold versus the absorption cross-section yield, $F_{\mathrm{th}}=0.8363 \sigma_{\text {abs }}^{-0.311}$ with $R^{2}=0.46$. Figure 4.14 shows the relation between the maximum field enhancement for different morphologies, wavelengths of laser irradiation and optical breakdown threshold. A power regression fit of the optical breakdown versus the maximum field enhancement yielded, $F_{\mathrm{th}}=44.96\left(E_{\mathrm{e}, \max }\right)^{-1.267}$ with $R^{2}=0.92$.

Figure 4.15 shows the change in the optical response of the $25 \mathrm{~nm}$ gold nanosphere trimer (s25t) when adding a hypothetical $2 \mathrm{~nm}$ thick homogeneous layer with refractive index of 1.6 around it. There is a $20 \mathrm{~nm}$ shift in the resonance wavelength for maximum near-field enhancement, $E_{\mathrm{e}, \max }$, and absorption cross-section, $\sigma_{\mathrm{abs}}$, and there is a $28 \%$ increase in $E_{\mathrm{e}, \max }$, and a $9 \%$ increase in $\sigma_{\mathrm{abs}}$ at the resonant wavelength due to the addition of the $2 \mathrm{~nm}$ thick layer around the nanospheres. Such changes in the optical response by the nanostructure will affect multiphoton absorption by the environment in the vicinity of gold nanoparticle and photo-thermal emission by the gold nanoparticle. Ideally these effects should be modelled, if they can be properly characterized. Unfortunately, the characterization of the immediate vicinity of nanostructures is very difficult.

In the calculations the size corrections to bulk dielectric function of gold [26] were used. To understand whether correction of bulk dielectric function of gold can effect the predicted optical breakdown thresholds, the maximum near-field enhancement and absorption cross-section were calculated for gold nanosphere trimers with different diameters and nanorods with same aspect ratios. Table 4.2 shows the relative change in $|\mathbf{E}|_{\max }$ and $\sigma_{\text {abs }}$ applying the corrections to the dielectric function of gold. As expected, 
(a) Maximum near-field enhancement

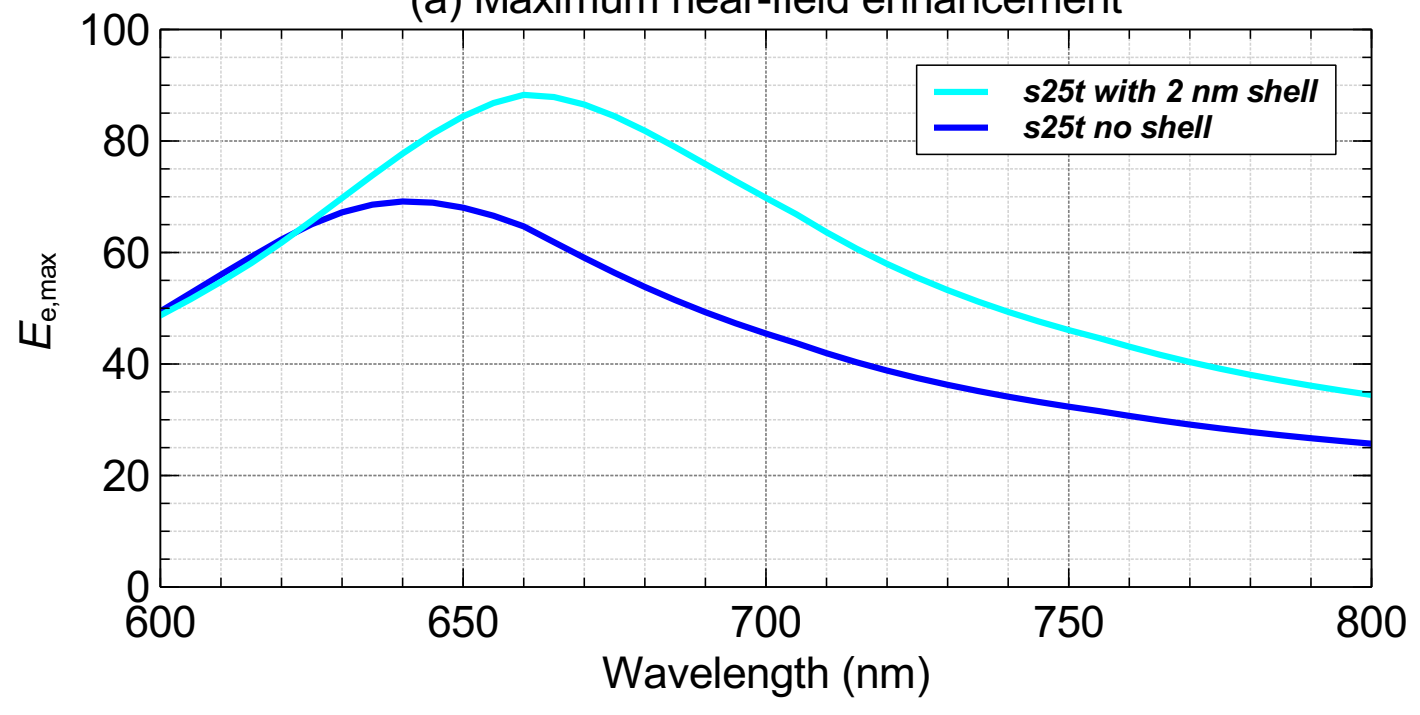

(b) Absorption cross-section

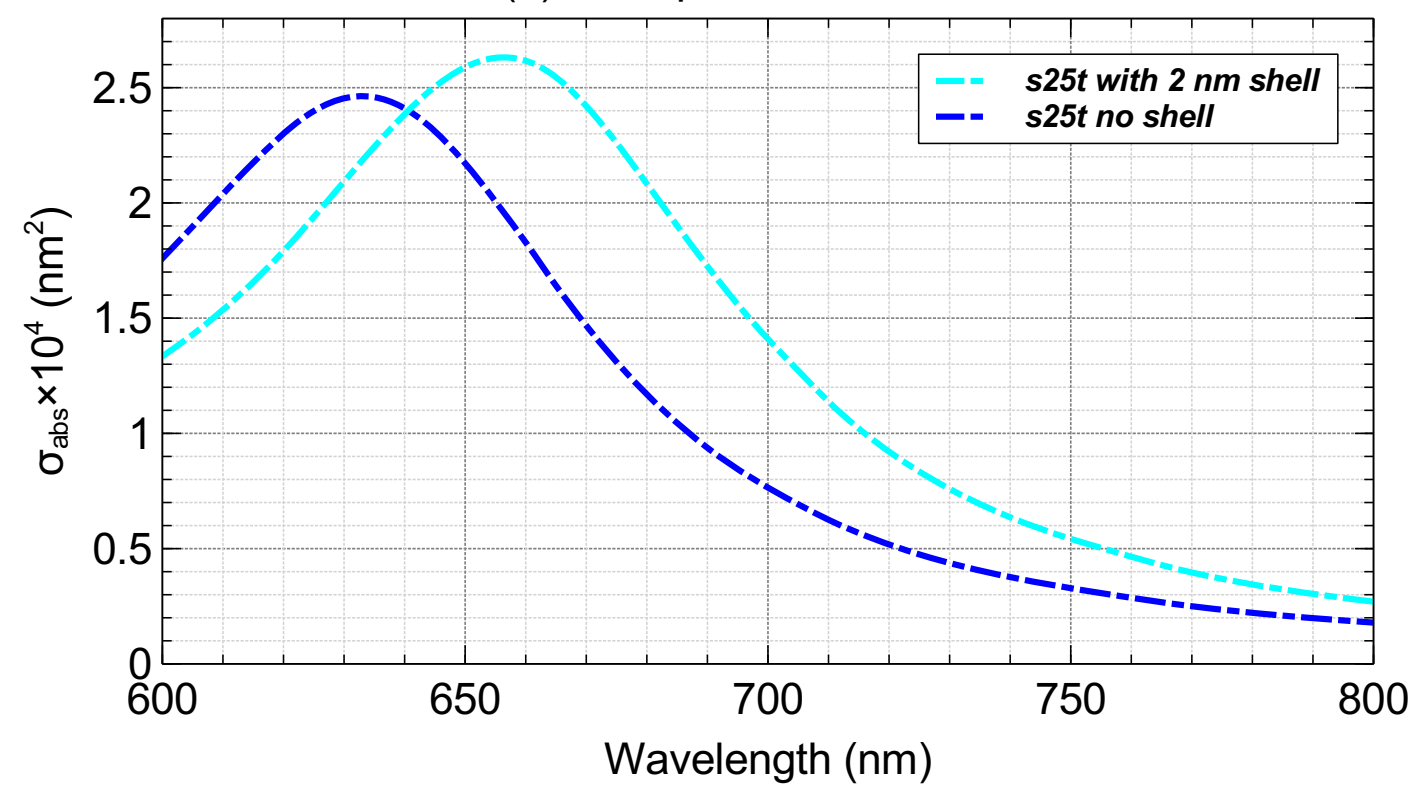

Figure 4.15: Maximum near-field enhancement, $E_{\mathrm{e}, \max }$, and absorption cross-section, $\sigma_{\text {abs }}$, of a $25 \mathrm{~nm}$ gold nanosphere trimer with and without inclusion of $2 \mathrm{~nm}$ thick shell with refractive index of 1.6. 
Table 4.2: Overestimation of the maximum near-field enhancement, $\left(|\mathbf{E}|_{\text {max bulk }}-\right.$ $\left.|\mathbf{E}|_{\max }\right) /|\mathbf{E}|_{\text {max,bulk, }}$, and absorption cross-section, $\left(\sigma_{\text {abs,bulk }}-\sigma_{\text {abs }}\right) / \sigma_{\text {abs,bulk }}$, by the gold nanosphere trimer and nanorod at longitudinal plasmon resonance wavelength with the use of bulk dielectric function of gold versus size corrected dielectric function of gold.

\begin{tabular}{|c|c|c|}
\hline Nanostructure & $\frac{|\mathbf{E}|_{\max , \text { bulk }}-|\mathbf{E}|_{\max }}{|\mathbf{E}|_{\max , \text { bulk }}}$ & $\frac{\sigma_{\text {abs,bulk }}-\sigma_{\text {abs }}}{\sigma_{\text {abs,bulk }}}$ \\
\hline s5t@560 & 0.17 & 0.165 \\
s15t@585 & 0.09 & 0.066 \\
s25t@640 & 0.04 & 0.003 \\
\hline r5t@780 & 0.40 & 0.397 \\
r15t@800 & 0.09 & 0.044 \\
r25t@930 & 0.02 & 0.002 \\
\hline
\end{tabular}

for a large nanostructures (s25t@640 and r25t@930) the optical properties did not change much due to inclusion of size corrected dielectric function of gold. On the other hand, for the smalest nanosphere (s5t@560) and nanorod (r5t@780) $|\mathbf{E}|_{\max }$ and $\sigma_{\text {abs }}$ changed by a factor of 0.17 and 0.40 , respectively. In such a cases, not usage of the size corrected dielectric function of gold will lead to underestimation of optical breakdown threshold.

\subsection{The wavelength dependence of gold nanorod-mediated optical breakdown with infrared ultrashort pulses}

For the wavelength dependence of the nanoparticle-mediated optical breakdown study, the interaction of a longitudinally polarized femtosecond (60 fs) and a picosecond (6 ps) laser pulse with a GNR having either an aspect ratio of $3.5\left(\mathrm{GNR}_{35} ; 10 \times 35 \mathrm{~nm}\right)$ or $5.5\left(\mathrm{GNR}_{55} ; 10 \times 55 \mathrm{~nm}\right)$ was used. These aspect ratios were chosen so that the GNRs had a plasmon peak positions of $750 \mathrm{~nm}$ and $975 \mathrm{~nm}$, respectively, which are close to the transition in the order of the MPI process, $\kappa$, (where an additional photon is needed to overcome the initiation energy, $E_{\text {ini }}$ ) for pure water of $738 \mathrm{~nm}$ (for transition zone between $\kappa=4$ and $\kappa=5$ ) and $965 \mathrm{~nm}$ (between $\kappa=5$ and $\kappa=6$ ) [73, 74].

The simulated absorption cross-section, $\sigma_{\text {abs }}$, and a maximum electric field enhancement, $E_{\mathrm{e}, \max }=\max |\mathbf{E}| / E_{0}$, where $\max |\mathbf{E}|$ is the maximum electric field in the vicinity of the nanoparticle and $E_{0}$ is the magnitude of the incident electric field for both GNRs are 
shown in Figure 4.16. As expected, the peak positions of $\sigma_{\mathrm{abs}}$ and $E_{\mathrm{e}, \mathrm{max}}$ are the same for each nanorod. In the absorption regime of LIOB the $\sigma_{\text {abs }}$ is an important parameter that affects the PTE rate, while in near-field regime of LIOB, $E_{\mathrm{e}, \max }$ is an important parameter that affects the MPI rate.

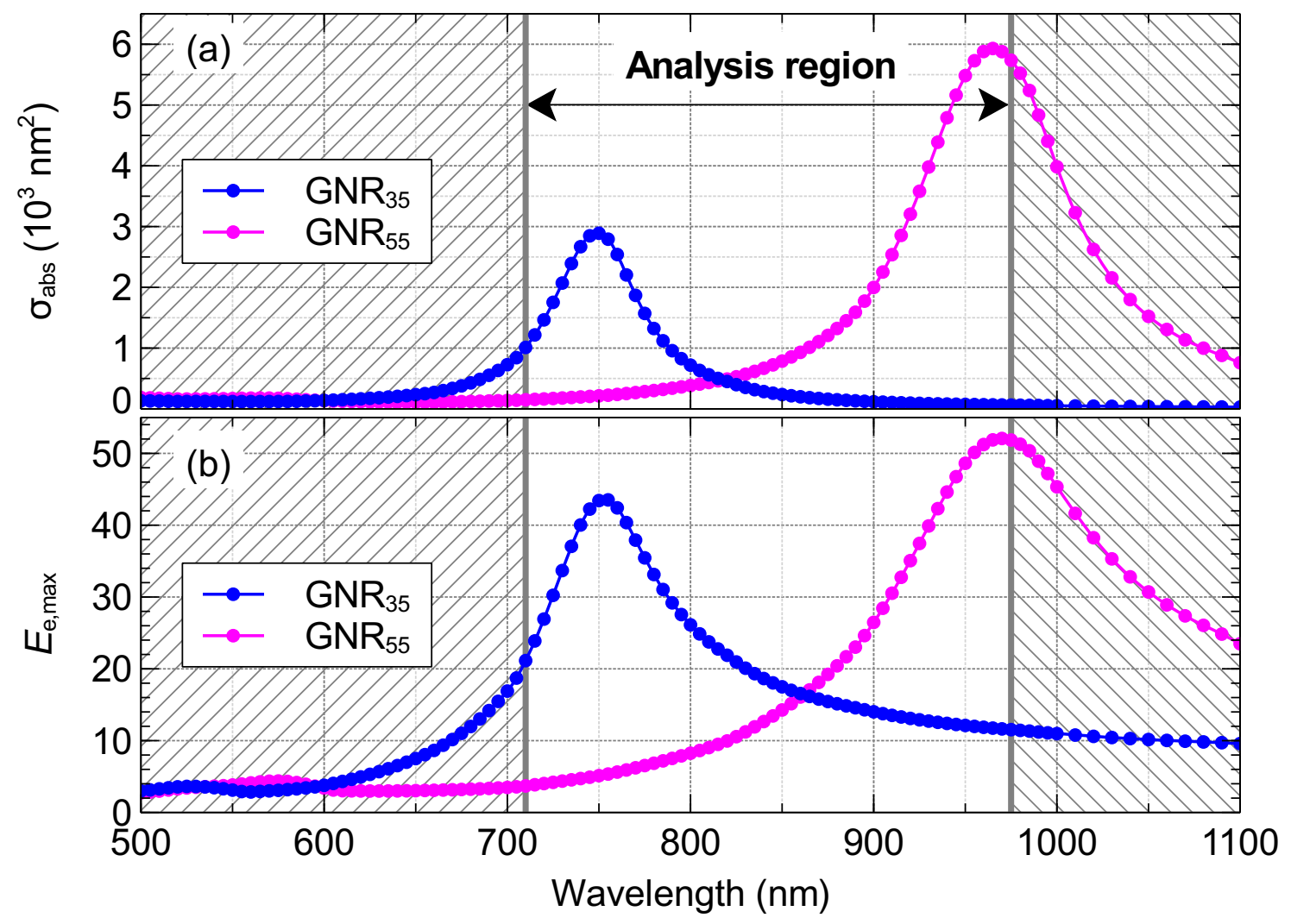

Figure 4.16: Absorption cross-section, $\sigma_{\text {abs }}(\mathrm{a})$ and a maximum electric field enhancement, $E_{\mathrm{e}, \max }(\mathrm{b})$ of a longitudinally polarized light of a $35 \mathrm{~nm}\left(\mathrm{GNR}_{35}\right)$ (green) and a $55 \mathrm{~nm}\left(\mathrm{GNR}_{55}\right)$ long gold nanorod (red).

For a given particle, due to the similar spectral shapes of $\sigma_{\text {abs }}$ and $E_{\mathrm{e}, \max }$, the relationship between $I_{\text {th }}$ and $\sigma_{\text {abs }}$ is expected to be the same as the relationship between $I_{\text {th }}$ and $E_{\mathrm{e}, \max }$ since $\sigma_{\mathrm{abs}}$ is directly proportional to magnitude of electric field. Either one could be increased to lower the optical breakdown threshold. Figure $4.17 \mathrm{a}$ shows the $I_{\text {th }}$ dependency on $\sigma_{\mathrm{abs}}$ for the 60 fs and $6 \mathrm{ps}$ pulses. It is evident that for a given pulse duration $I_{\text {th }}$ depends on $\sigma_{\text {abs }}$ independent of the GNR aspect ratio. $I_{\text {th }}$ also depends on $E_{\mathrm{e}, \max }$ but this dependency is different for the two aspect ratios and pulse durations 
(Figure 4.17b,c).

(a)

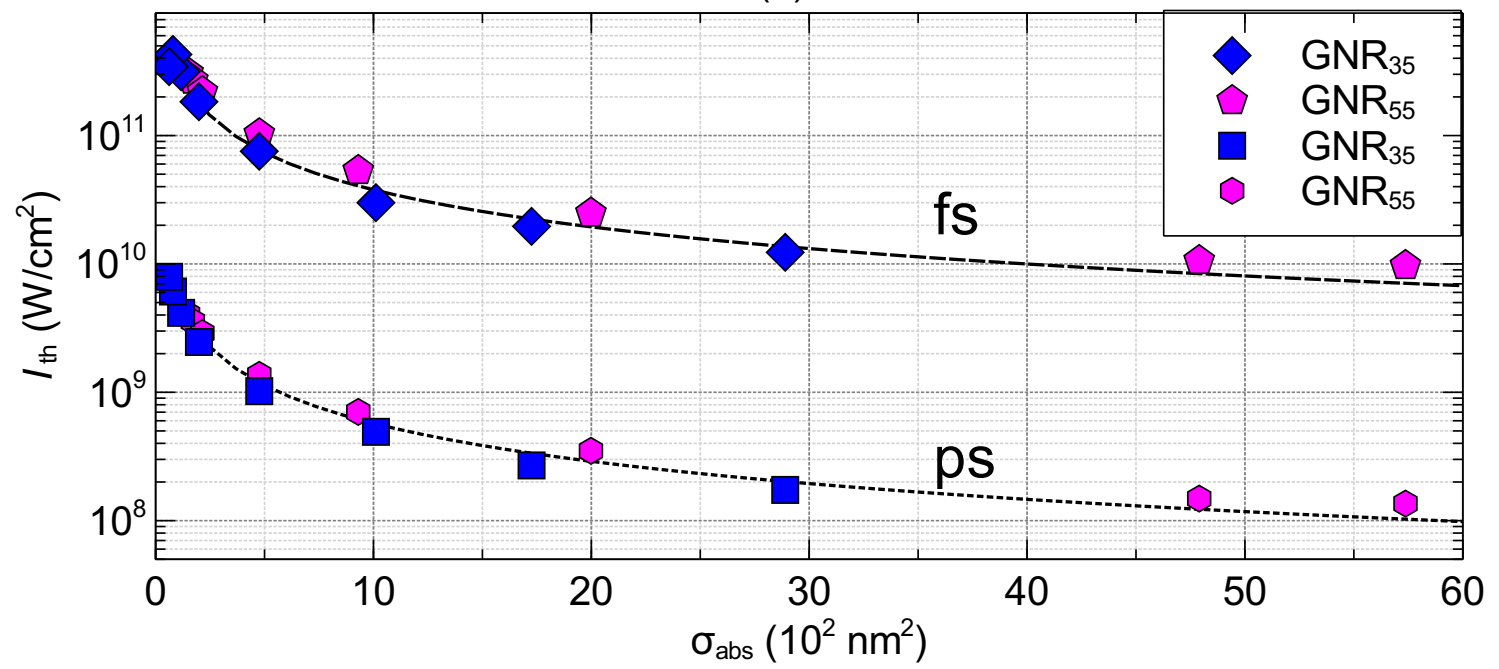

(b)

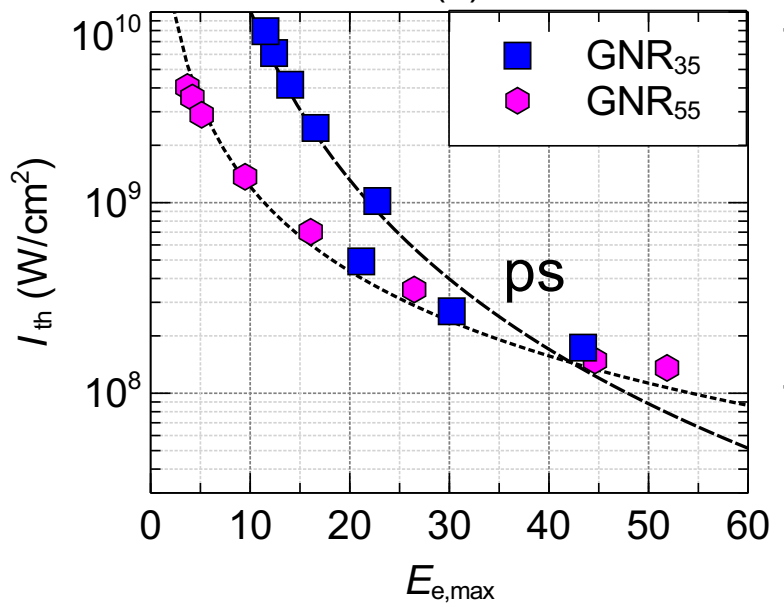

(c)

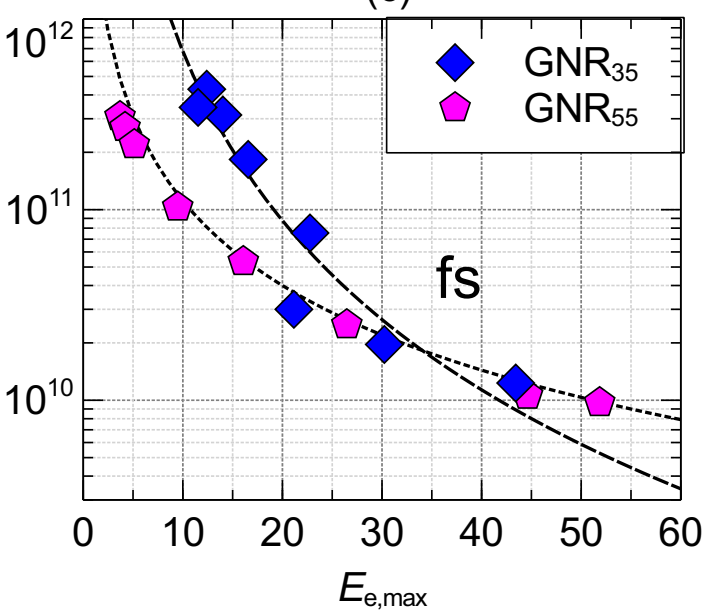

Figure 4.17: $I_{\text {th }}$, LIOB intensity threshold for a 60 fs and 6 ps laser pulses versus (a) absorption cross-section, $\sigma_{\text {abs }}$, of the 35 and $55 \mathrm{~nm}$ GNR and a maximum electric field enhancement, $E_{\mathrm{e}, \max }$, for a (b) 6 ps and (c) 60 fs pulse duration. Data power trend lines fit: (a) $I_{\text {th }}=2.87 \times 10^{13} \sigma_{\text {abs }}^{-0.96}$ (dashed line), and $I_{\text {th }}=5.1 \times 10^{11} \sigma_{\text {abs }}^{-0.984}$ (dotted line); (b) $I_{\text {th }}=9.05 \times 10^{12} E_{\mathrm{e}, \max }{ }^{-2.95}$ (dashed line) and $I_{\text {th }}=36.2 \times 10^{9} E_{\mathrm{e}, \max }{ }^{-1.475}$ (dotted line); (c) $I_{\text {th }}=6.03 \times 10^{14} E_{\mathrm{e}, \max }{ }^{-2.95}$ (dashed line) and $I_{\text {th }}=33.2 \times 10^{11} E_{\mathrm{e}, \max }{ }^{-1.475}$ (dotted line).

Figure $4.18 \mathrm{a}$ and $4.18 \mathrm{~b}$ shows the wavelength dependence of optical breakdown thresholds, $I_{\mathrm{th}}^{6 \mathrm{ps}}$ and $I_{\mathrm{th}}^{60 \mathrm{fs}}$, for a $6 \mathrm{ps}$ and $60 \mathrm{fs}$ pulse, respectively. The optical breakdown 
threshold for the picosecond and femtosecond pulses intersect at an $825 \mathrm{~nm}$ wavelength for both GNRs. This is the same point as the $\sigma_{\text {abs }}$ intersection (Figure 4.16a), in contrast to the intersection of $E_{\mathrm{e}, \max }$, which is at $860 \mathrm{~nm}$ (Figure 4.16b). This is consistent with Figure $4.17 \mathrm{a}$ where $I_{\text {th }}$ is well correlated with $\sigma_{\text {abs }}$ for a given pulse duration. As expected, for LIOB with a picosecond pulse exposure (where the absorption regime dominates) the wavelength dependence of $I_{\mathrm{th}}^{6 \mathrm{ps}}$ correlates with the wavelength dependence of $\sigma_{\mathrm{abs}}$ and $E_{\mathrm{e}, \max }$ (Figure 4.18a versus Figure 4.16. $I_{\mathrm{th}}^{6 \mathrm{ps}}$ is minimum at $750 \mathrm{~nm}$ for $\mathrm{GNR}_{35}$ where $\sigma_{\text {abs }}$ and $E_{\mathrm{e}, \max }$ are maximum, and for $\mathrm{GNR}_{55}$ a $I_{\mathrm{th}}^{6 \mathrm{ps}}$ is minimum around $950 \mathrm{~nm}$ while $\sigma_{\text {abs }}$ and $E_{\mathrm{e}, \max }$ are maximum at $975 \mathrm{~nm}$. For femtosecond pulses, the shape of the $I_{\mathrm{th}}^{60 \mathrm{fs}}$ resembles the shape of an $I_{\mathrm{th}}^{6 \mathrm{ps}}$ for $\mathrm{GNR}_{55}$ (Figure 4.18), with no evidence of a wavelength dependence due to the band gap structure of water, and its associated ionization transition zones [74]. However, $I_{\text {th }}^{60 f s}$ for the $\mathrm{GNR}_{35}$ decreases between $950 \mathrm{~nm}$ and $975 \mathrm{~nm}$, which is not correlated to changes in $\sigma_{\mathrm{abs}}$ or $E_{\mathrm{e}, \max }$ in this range (Figure 4.16). The peak is located near the transition zone of the MPI process, between $\kappa=5$ and $\kappa=6$. At this transition zone, the lower MPI process order, $\kappa=5$, which just exceeds $E_{\text {ini }}$, mixes with a higher order but a lower probability MPI process, $\kappa=6$, which becomes relatively important with increasing wavelength [74].

The absence of a maxima for $I_{\text {th }}^{60 f s}$ for $\mathrm{GNR}_{55}$ near the MPI order transition zone between $\kappa=4$ and $\kappa=5$, which is around $740 \mathrm{~nm}$, was analyzed by comparing the spacial distribution of the electric field enhancement, $E_{\mathrm{e}}=|\mathbf{E}| / E_{0}$, for both GNRs at a wavelength near the two MPI transition zones $(750 \mathrm{~nm}$ for the transition between $\kappa=4$ and $\kappa=5$ and $975 \mathrm{~nm}$ for the transition between $\kappa=5$ and $\kappa=6$ ). Figure 4.19 a-d shows this comparison. The spatial distribution of $E_{\mathrm{e}}$ is distinctly different for $\mathrm{GNR}_{55} @ 750 \mathrm{~nm}$ compared to the three other nanorods. For GNR ${ }_{55} @ 750 \mathrm{~nm}$ the highest $E_{\mathrm{e}}$ in the medium is located along the sides of the GNR, while for the others the maximum $E_{\mathrm{e}}$ in the medium is located at the ends of the GNR. If the MPI rate during seed electron density generation is higher than the PTE rate, then the temporal shape of free electron density, $\rho_{\mathrm{e}}$, at this point will resemble the temporal shape of the laser pulse intensity (green line, Figure $4.20 \mathrm{~b}$ ). This usually occurs at locations in the medium where $E_{\mathrm{e}}$ is much higher than $E_{\mathrm{e}}$ in the GNR. On the other hand, if the PTE rate is higher than the MPI rate, then the free electron density will increase rapidly during the pulse duration followed by a gradual decrease of free electrons after the end of the pulse (red line, Figure 4.20a). This is especially true during picosecond pulses at all wavelengths studied (Figures $4.18 \mathrm{a}$ 


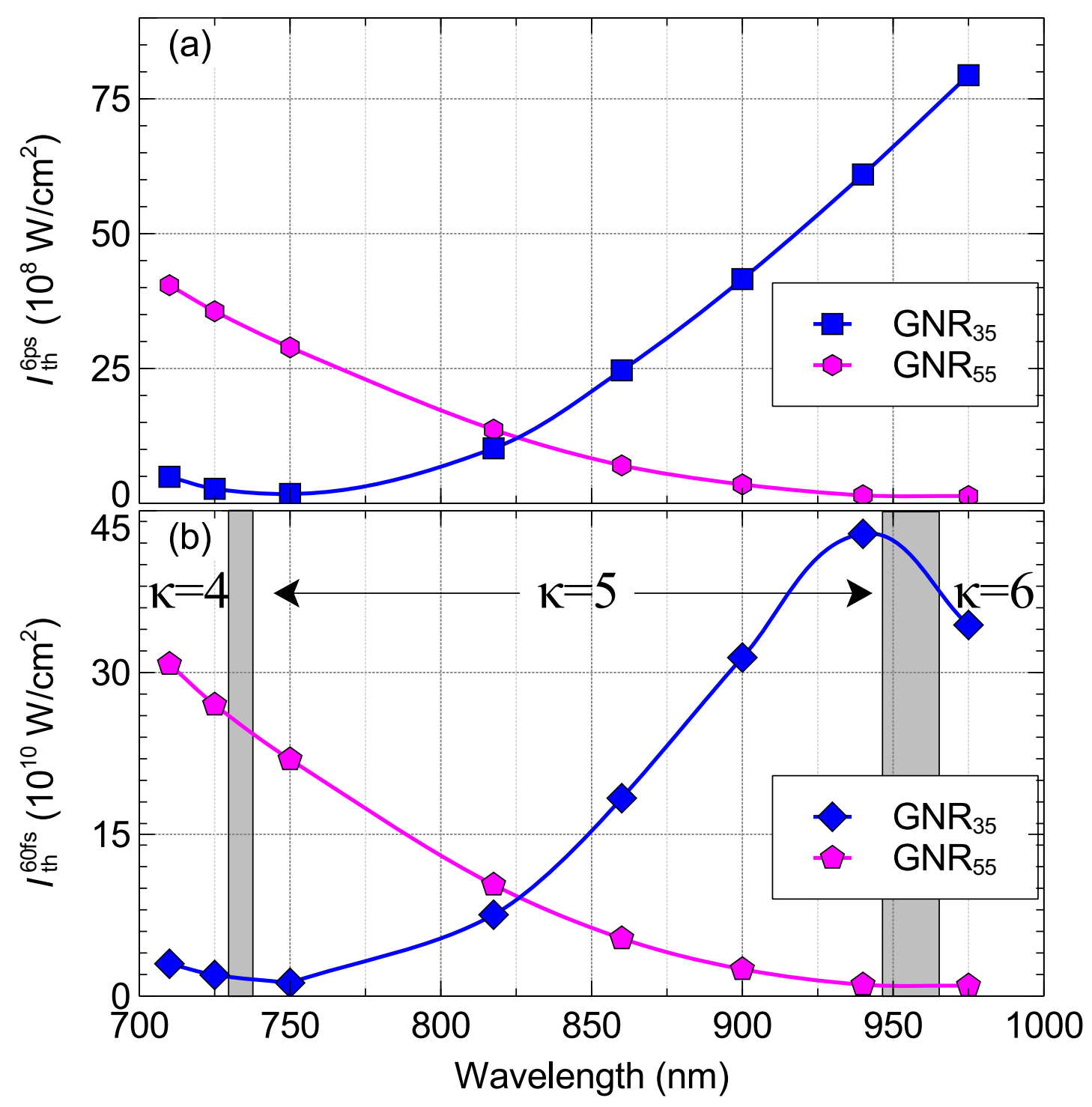

Figure 4.18: LIOB threshold $I_{\mathrm{th}}^{6 \mathrm{ps}}$ for a 6 ps and $I_{\mathrm{th}}^{60 \mathrm{fs}}$ for a 60 fs pulse interaction with a GNR versus the wavelength of incidence plotted for a $\rho_{\text {crit }}=1 \times 10^{21} \mathrm{~cm}^{-3}$. Grey shaded area marks the MPI process transition zones. $\kappa$ denotes the order of the MPI process required to cross the band gap of water [66].

and $4.20 \mathrm{a}$ ). Since $E_{\mathrm{e}}$ at the sides of $\mathrm{GNR}_{55} @ 750 \mathrm{~nm}$ in water is approximately equal to $E_{\mathrm{e}}$ inside the GNR (Figure 4.19p), it was expected that PTE off the GNR surface would dominate over MPI in the water. The dominance of PTE in seed electron generation during the femtosecond pulse interaction with $\mathrm{GNR}_{55}$ between 710 to $750 \mathrm{~nm}$ will cancel 
the wavelength dependence of $I_{\text {th }}$ caused by transitions in the MPI process order.

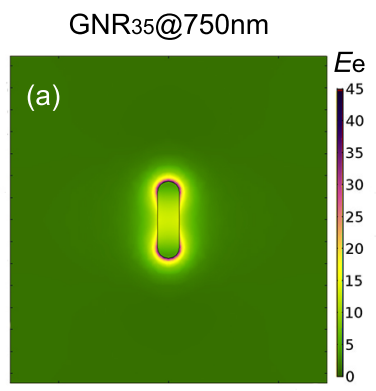

\section{GNR55@750nm}
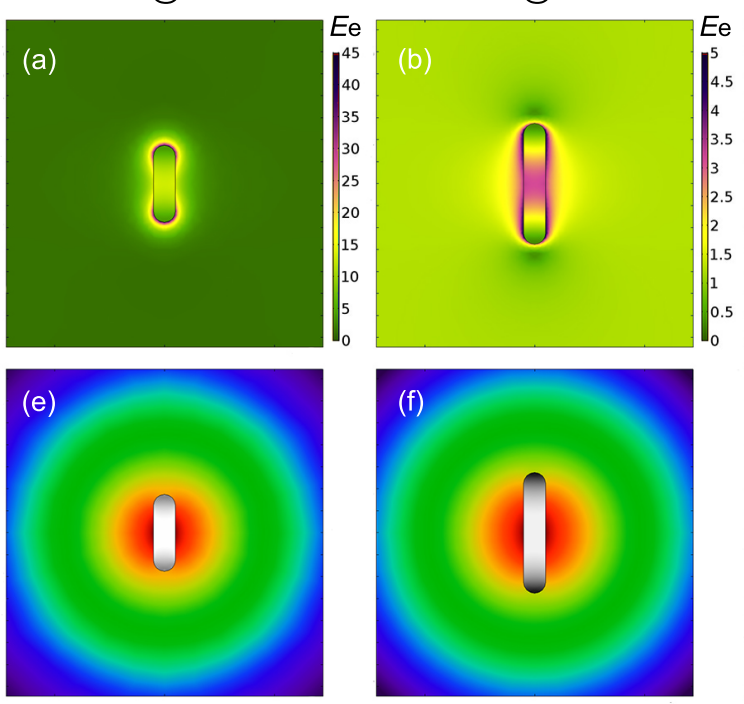

GNR35@975nm

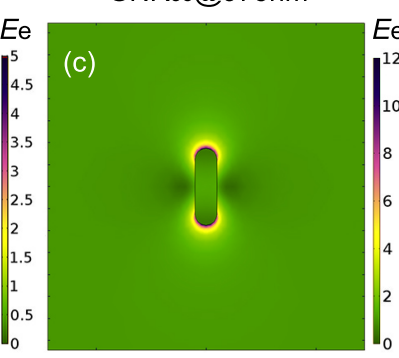

(g)
GNR55@975nm
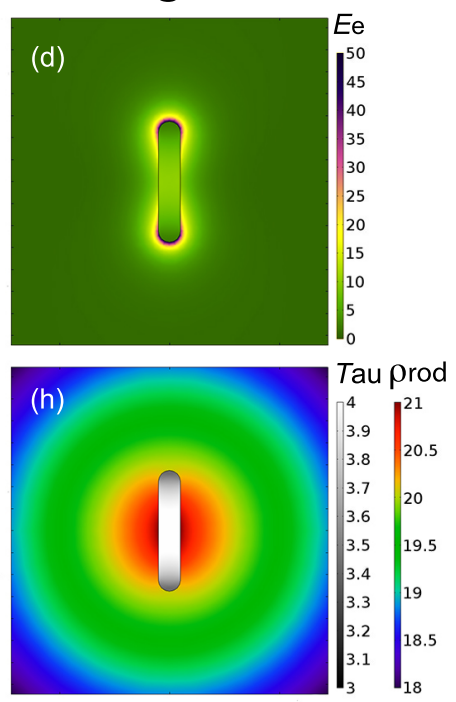

Figure 4.19: (a)-(d) Electric near field enhancement, $E_{\mathrm{e}}=|\mathbf{E}| / E_{0}$, plotted for $\mathrm{GNR}_{35}$ and $\mathrm{GNR}_{55}$ at 750 and $975 \mathrm{~nm}$ wavelengths, before formation of the free electron plasma that at $\rho_{\text {crit }}$ can shield the particle from incoming radiation. (e)-(h) Gold nanorod's electron temperature, $T_{\mathrm{au}}=\log _{10}\left(T_{\mathrm{e}}[K]\right)$, is plotted in the $\log$ scale at the time of peak irradiance of the 60 fs laser pulse (grey scale colour bar). The log scale of a free electron plasma density, $\rho_{\text {rod }}=\log _{10}\left(\rho_{\mathrm{e}}\left[\mathrm{cm}^{-3}\right]\right)$, in the vicinity of the GNR at the time when $\rho_{\mathrm{e}}=\rho_{\text {crit }}$ is reached.

The existence of high $E_{\mathrm{e}}$ near the surface of a GNR does not necessary mean that free electron generation is dominated by MPI. For example, the similar $E_{\mathrm{e}}$ distributions for GNRs in Figure 4.19k, d produce vastly different $\rho_{\mathrm{e}}$ distribution (Figure $4.19 \mathrm{~g}, \mathrm{~h}$ ). Also, similar $\rho_{\mathrm{e}}$ distributions (Figure 4.19, f) were produced for GNRs with vastly different $E_{\mathrm{e}}$ distributions( Figure $4.19 \mathrm{a}, \mathrm{b})$.

Figure 4.21 shows $\rho_{\mathrm{e}}$ for a 60 fs pulse at different wavelengths for $\mathrm{GNR}_{35}$ and $\mathrm{GNR}_{55}$. This helps us understand how the MPI and PTE processes contribute to the free electron plasma generation in the GNR's vicinity. The figure shows the temporal profile of $\rho_{\mathrm{e}}$ at sampling points indicated in Figure $4.20 \mathrm{~b}$. MPI dominates free electron generation over PTE at the ends of the GNR if the MPI generated $\rho_{\mathrm{e}}$ (green curve in Figure 4.21) reaches $\rho_{\text {crit }}$ before the PTE generated $\rho_{\mathrm{e}}$ reaches $\rho_{\text {crit }}$ (red line). PTE dominates the production of seed electrons at the sides of the GNR, when PTE generated $\rho_{\mathrm{e}}$ reaches $\rho_{\text {crit }}$ (red line), 
(a)

\section{GNR ${ }_{35} @ 940 \mathrm{~nm}$}

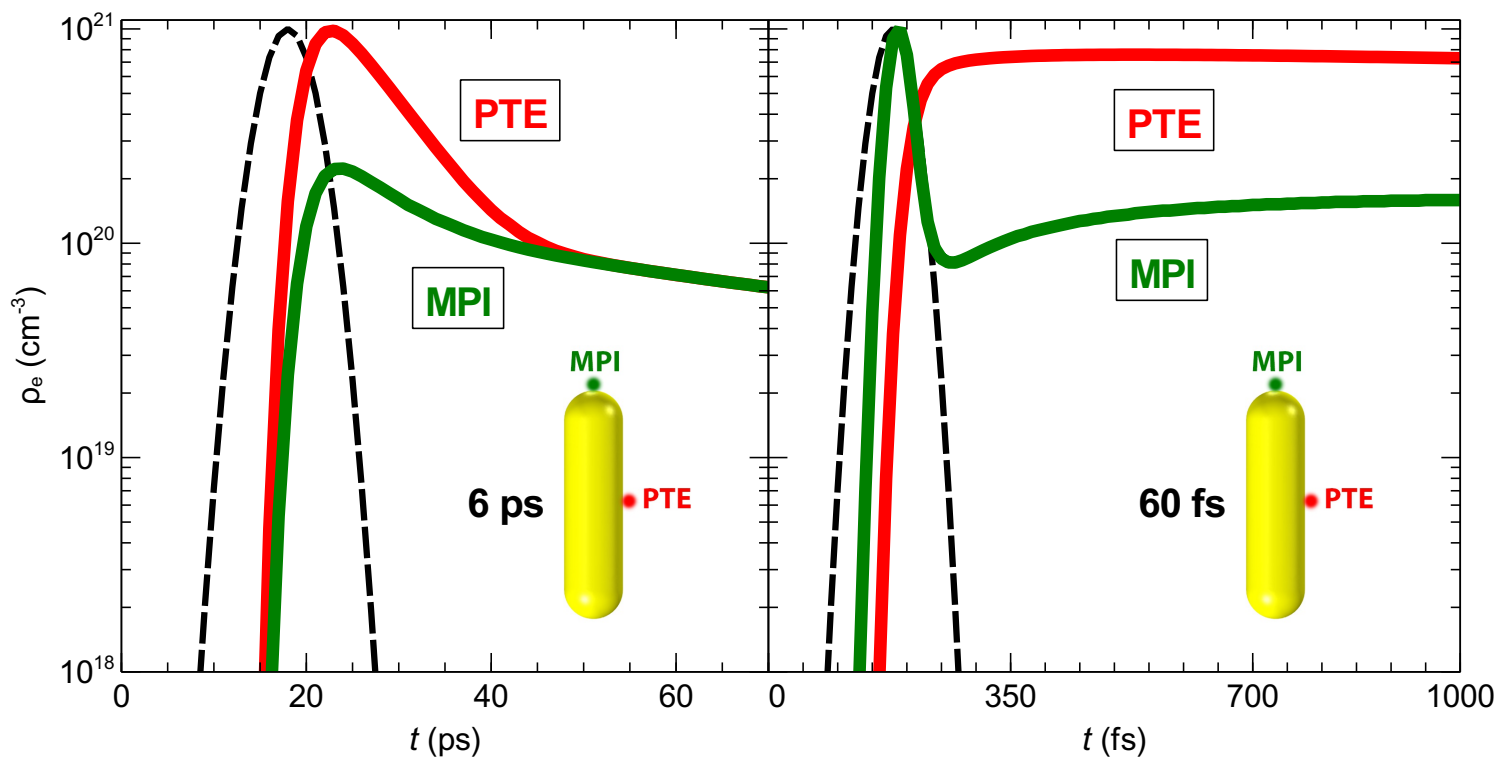

Figure 4.20: (a), (b) plots the free electron density, $\rho_{\mathrm{e}}$, generated in the vicinity of $\mathrm{GNR}_{35}$ exposed at $940 \mathrm{~nm}$ during $6 \mathrm{ps}$ and $60 \mathrm{fs}$ pulses, respectively. The sampling points of electron density lines are related dominant mechanisms (green line to MPI and red line to PTE) of free electron generation. The dashed line shows the temporal shapes of the $6 \mathrm{ps}$ and 60 fs laser pulses for (a) and (b), respectively.

before the MPI generated $\rho_{\mathrm{e}}$ (green line) reaches $\rho_{\text {crit }}$. At the ends of the GNR (where MPI dominates), the shape of the free electron density line (the green curve) follows the temporal distribution of the laser pulse irradiance. By examining the green curve in Figure $4.20 \mathrm{~b}$, one can see that after the peak of the laser pulse irradiance, the free electron production at the ends of the GNR due to the MPI decreases and free electron generation is overtaken by free electron production at the sides of the GNR (where PTE dominates). This can be seen by the gradual increase of the free electron density after the dip in the green MPI lines in Figures 4.21 $\mathrm{a}-\mathrm{h}$ and 4.21 -p. The free electron density line that results from PTE rises rapidly as the electron temperature of the GNR increases and $\rho_{\mathrm{e}}$ reaches its maximum by the end of the laser pulse (Figure 4.21).

By analyzing Figure 4.21, it is evident that for both GNRs the free electron plasma production is dominated by PTE at all examined wavelengths with the exception of 
$\mathrm{GNR}_{35}$ exposed at 940 and $975 \mathrm{~nm}$ wavelengths (Figure 4.21, $\mathrm{h}$ ), where the MPI process contributes strongly to the free electron plasma generation, and $\rho_{\mathrm{e}}=\rho_{\text {crit }}$ is reached at the ends before it is reached at the sides of the GNR (where PTE dominates). The dominant role of PTE for a femtosecond pulse is surprising since it is generally assumed that the near-field enhancement by a nanoparticle will provide more free electrons through MPI rather than through PTE during ultra-fast femtosecond exposures [82]. $E_{\mathrm{e}, \max }$ in the vicinity of the GNR is not sufficient by itself for determining whether nanoparticlemediated LIOB in water occurs through the near-field enhancement (MPI dominated) or absorption (PTE dominated) regimes. For example, $E_{\mathrm{e}, \max }$ for $\mathrm{GNR}_{55}$ is 3.6 and 4.5 times higher than for $\mathrm{GNR}_{35}$ at 940 and $975 \mathrm{~nm}$, respectively (Figure 4.16p), yet the overall free electron plasma for $\mathrm{GNR}_{55}$ is dominated by PTE (Figure 4.21p,p). This behaviour is due to the fact that a high field enhancement outside of the nanoparticle is accompanied by a high electric field enhancement inside of the GNR, leading to high absorption and PTE rates.

The ratio of the maximum electric field outside the GNR to the maximum electric field inside the GNR, $E_{\text {ratio }}=|\mathbf{E}|_{\text {max,outside }} /|\mathbf{E}|_{\text {max, inside, was investigated as a parameter to }}$ characterize the role MPI (near-field LIOB regime) and PTE (absorption LIOB regime) in free electron plasma generation. As is evident in Figure 4.21 for $\mathrm{GNR}_{35}$ at $940 \mathrm{~nm}$, $\rho_{\mathrm{e}}=\rho_{\text {crit }}$ is reached in the near-field regime (peak of the green line in Figure 4.21 $\mathrm{g}$ ), which is partially due to high near-field enhancement (high MPI rate), and partially due to the off-resonance excitation of $\mathrm{GNR}_{35}$ (low absorption and PTE rate). Hence, a high $E_{\text {ratio }}$ corresponds to lower absorption rates which causes a lowering and delaying of the PTE contribution to the plasma density generation (red line in Figures $4.21 \mathrm{a}-\mathrm{h}$ ). The near-field regime becomes evident during the free electron generation at $E_{\text {ratio }} \approx 7$ (Figure $4.21 \mathrm{~g}$ ). On the other hand, for $\mathrm{GNR}_{55}$ the relative contribution of PTE to the free electron generation remains strong at the MPI transition zone near $975 \mathrm{~nm}$ (red line in Figures $4.21 \mathrm{p}-\mathrm{p}$ ). This comes from the fact that the plasmon resonance peak position for $\mathrm{GNR}_{55}$ is located at this wavelength, which results in an increase of $\sigma_{\text {abs }}$ (Figure 4.16) and $E_{\mathrm{e}}$ inside the GNR. The increase in the $\sigma_{\mathrm{abs}}$ and $E_{\mathrm{e}}$, inevitably leads to an increase in resistive heating and consequently PTE. Therefore $E_{\text {ratio }}=7$ indicates a threshold for switching from absorption to the near-field regime for free electron generation during femtosecond pulse exposures. An $E_{\text {ratio }} \geq 7$ also indicates the point where changes in the MPI process order can affect $I_{\text {th }}$. 


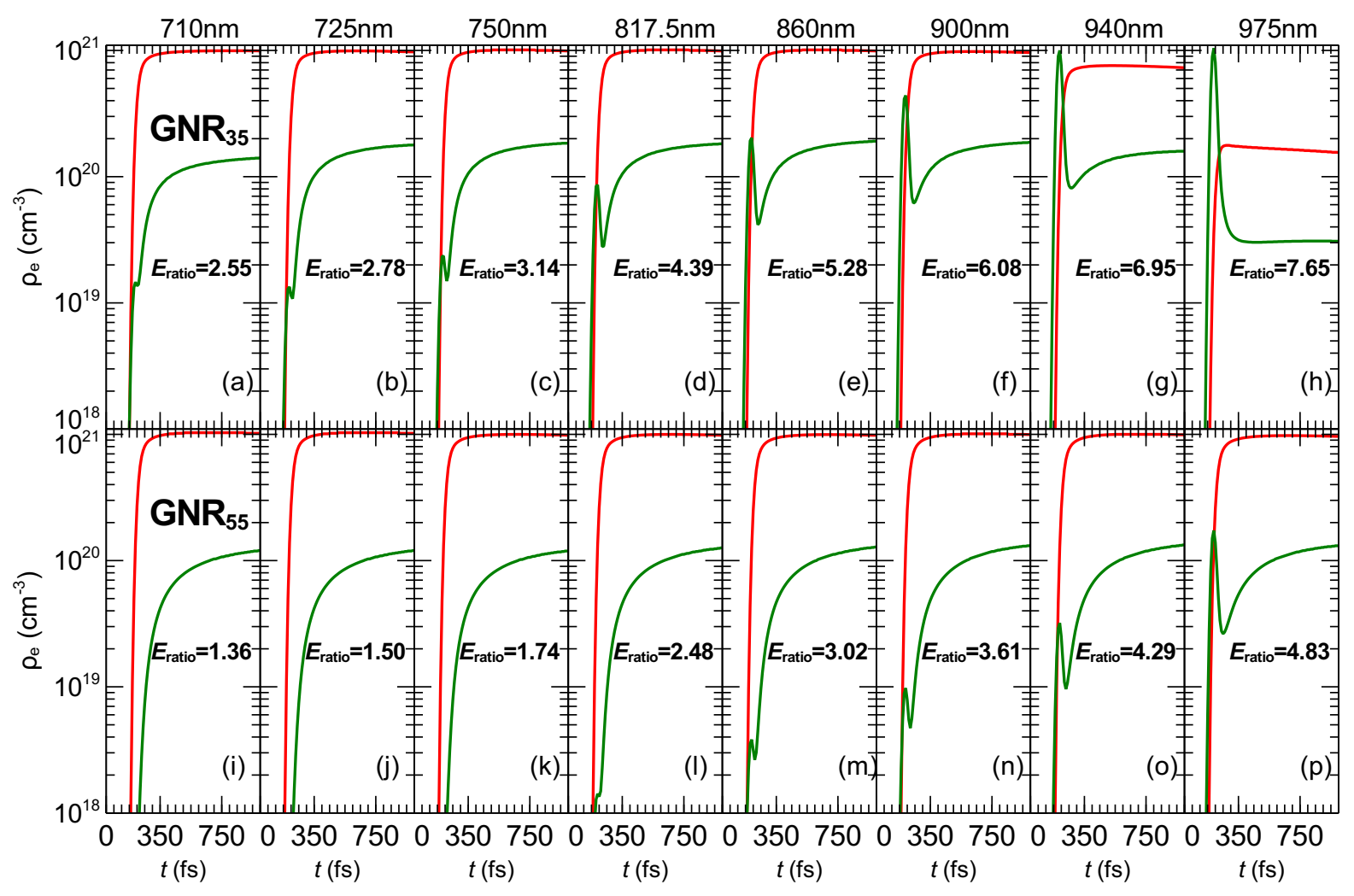

Figure 4.21: Free electron plasma density, $\rho_{\mathrm{e}}$, generation related to contribution to the free electron plasma generation through the MPI - green line (sampled at the tip of the GNR, see Figure 4.20) and PTE - red line (sampled at the surface of the GNR in the middle point of GNR, see Figure 4.20 processes in the vicinity of $\mathrm{GNR}_{35}$ and $\mathrm{GNR}_{55}$ during a $60 \mathrm{fs}$ laser pulse interaction, respectively. $E_{\text {ratio }}$ shows the ratio of maximum electric field outside to inside of the GNR. 



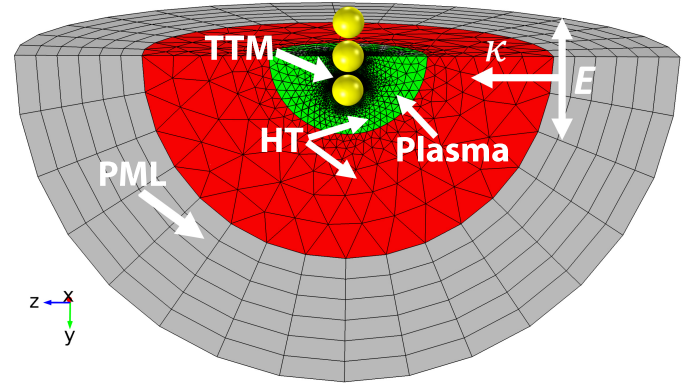

Summary, Discussion and Conclusions

The focus of this thesis was the development of a finite element model of a nanoparticlemediated optical breakdown phenomena in water. COMSOL Multiphysics was used to build a strongly coupled complex multiphysics phenomena of optical breakdown due to strong field ionization of water in the presence of gold nanoparticles. The latest findings on the water's band structure were incorporated into the model in order to update the current state of the computational formulation of nanoparticle-mediated optical breakdown. A summary and conclusions for the validation of the model and the three studies are provided below.
\end{abstract}

\title{
5.1 Validation of LIOB model
}

A thorough model of nanoparticle-mediated optical breakdown was developed. The model was used to predict the optical behaviour of a gold nanoshell and a strong field ionization of water during 70 fs pulse exposure in order to validate it against the experimental data published by Lachaine et al. [97.

The validation results that were presented in the Section 4.1 .3 demonstrated a good agreement with experimentally observed data published by Lachaine et al. 97]. For one gold nanoshell, NS800, the predicted bubble formation threshold was around $7 \mathrm{~mJ} / \mathrm{cm}^{2}$ and in agreement with the experiment, while for another gold nanoshell, NS660, the predicted bubble formation threshold was at $15 \mathrm{~mJ} / \mathrm{cm}^{2}$ which is almost a half of what was observed experimentally $\left(35 \mathrm{~mJ} / \mathrm{cm}^{2}\right)$. This disagreement would be caused by limitations 
in bubble detectability. The electron density threshold for bubble formation for pure water noted in Figures 4.4 and 4.5 , were based on the formation of bubbles that were at least $150 \mathrm{~nm}$ in radius [75]. Lachaine et al. [97] were only able to detect bubbles that were at least $250 \mathrm{~nm}$ in radius. This uncertainty is further highlighted by experiments with live cells, where the optoporation was observed at $20 \mathrm{~mJ} / \mathrm{cm}^{2}$ fluence (in contrast to $35 \mathrm{~mJ} / \mathrm{cm}^{2}$ fluence threshold for bubble formation) for NS660 nanoshell (see Figure 5.1). On the other hand, optoporation was observed with NS800 particles at fluence above $20 \mathrm{~mJ} / \mathrm{cm}^{2}$ (in contrast to $6 \mathrm{~mJ} / \mathrm{cm}^{2}$ fluence threshold for bubble formation). The variation in experimentally observed thresholds of bubble formation versus optoporation efficacy can be understood by noting that optoporation of live cell can be caused by a single large bubble (as in the case of NS800, where only single bubbles with a diameter of $\approx 2 \mu \mathrm{m}$ at $20 \mathrm{~mJ} / \mathrm{cm}^{2}$ were observed when exposed to a sequence of laser pulses) or a sequence of smaller bubbles (as in the case of NS660, where exposure to a sequences of laser pulses generated a sequence of bubbles, with diameters of less than $0.5 \mu \mathrm{m}$ at fluences below $40 \mathrm{~mJ} / \mathrm{cm}^{2}$ ).

An explanation for the variation in the experimentally observed thresholds for bubble formation is that the definition of a bubble formation threshold that is based on the maximum free electron plasma density for pure water is inadequate for the bubble formation threshold for gold nanoparticle-mediated optical breakdown in water. Optical breakdown in water can be associated with two phenomena: transition of the water from a liquid to a gas phase (caused by energy deposition) and luminescence by the plasma. The transition to the gas phase (cavitation and bubble formation) during optical breakdown is easily detectable. The temperature of water can be related to the threshold of bubble formation and does not depends on the laser pulse duration. On the other hand, the temperature of the plasma can be related to the threshold of luminescence, but it depend on the laser pulse duration. The brightness of the luminescence strongly depends on the plasma temperature. The use of temperature as an alternative for bubble formation during nanoparticle-mediated optical breakdown is discussed in Section 6.1 where a new criterion for optical breakdown that includes the temperature of water is provided. 

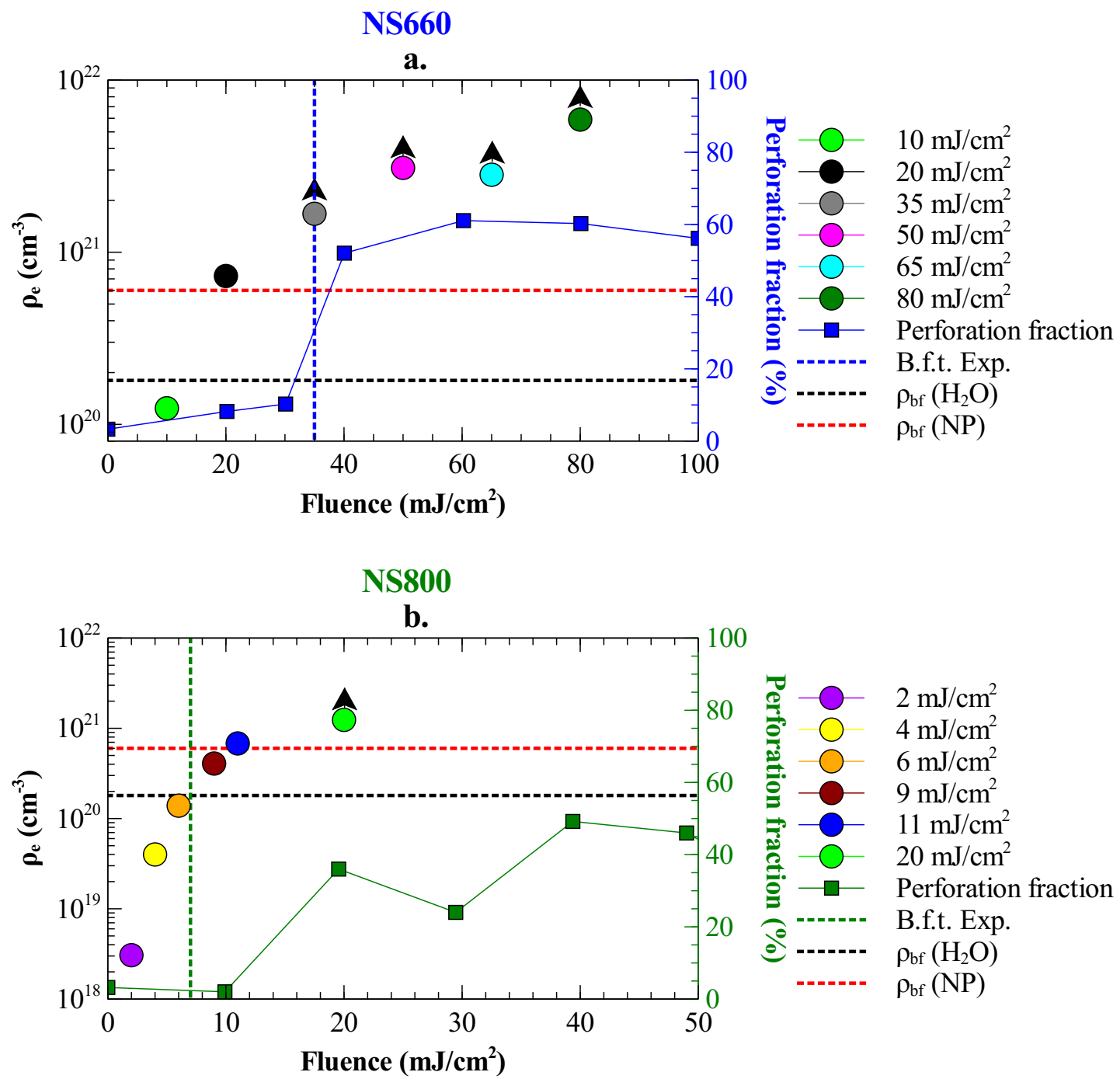

Figure 5.1: A comparison of the maximum free electron density calculated in the vicinity of gold nanoshells (circles) with the perforation fraction (\%) blue and green squares for a. NS660 b. NS800, respectively. The black arrows indicates models where calculation did not reach the end of the $70 \mathrm{fs}$ pulse. Blue and green dashed line represents experimental bubble detection threshold for NS660 and NS800, respectively. Black dashed line represents a bubble formation threshold for a femtosecond pulse in water [75] and set as a $\rho_{\mathrm{bf}}=1.8 \times 10^{2} \mathrm{~cm}^{-3}$. Red dashed line represents redefined maximum free electron density in the vicinity of a nanoshell needed to reach a bubble formation threshold based on Figure 6.1 (see text for details). 


\subsection{Role of environment on optical properties of a single nanoparticle}

The optical extinction spectra of single bare and silica-coated gold nanorods were quantitatively measured around their longitudinal SPR using spatial modulation spectroscopy and the results compared to those of a numerical model of optical absorption, taking into account a realistic experimental geometry and the actual nanoparticle morphology and size measured by transmission electron microscopy (extinction is dominated by absorption for the investigated nanorod sizes). The combination of these experimental and theoretical methods constitutes a powerful tool for the detailed interpretation of the optical properties of single nanoparticles. Excellent agreement between the computed and experimental data, that is, surface plasmon resonance wavelength and extinction cross section amplitude, have been obtained for the silica-coated rods, assuming they are deposited on a silica layer in air with the use the TEM measured rod dimensions as input. Conversely, a similar analysis with bare rods produced a large deviation of the computed longitudinal SPR wavelengths as compared to the experimental ones due to their greater sensitivity to their actual environment. Although a better reproduction of the measurements can be obtained using a mean homogeneous environment approximation, which has been successfully applied to the investigation of single nanospheres, this has been found to mask the actual complexity of the particle environment in single

particle measurements. Improved agreement with the optical measurements can also be obtained using a more realistic model assuming that the single nanoparticles deposited on the substrate are embedded within a thin water drop, the latter mimicking the impact of residual solvent, surfactant molecules, or humidity.

These results stress the difficulty in properly describing the measured optical response of a metal nanoparticle deposited onto a substrate, a geometry that is often used in single nanoparticle studies. This problem can be partly solved by embedding the particle into a dielectric layer (e.g., polymer film [88]) or dielectric shell to insulate it from uncontrolled environmental conditions. The effects of contamination by a water layer could be avoided by performing single particle spectroscopic measurements in a vacuum chamber. The influence of the local environment on the measured optical properties of single nanoparticles and of the particle to particle variation is similar to that encountered in 
the investigation of the damping of the acoustic vibration of nanoparticles [168]. This stresses the importance of controlling the local particle environment in single nanoparticle studies, which is efficiently done with silica-coated particles, particularly when the particles have complex shapes.

\subsection{Morphology dependence of LIOB}

The optical breakdown threshold for a picosecond pulse interaction with gold nanoparticles of different morphologies was shown to be highly dependent on near-field enhancement in the nanoparticle vicinity and to a lesser degree on the nanoparticle type, volume and absorption cross-section. In the case of uncoupled nanoparticles, the optical breakdown threshold is highly dependent on both the absorption cross-section and the nearfield enhancement by the nanoparticle due to the similarity in their spectral shapes. The results obtained show that the use of nano-assemblies can lower the threshold by 4 orders of magnitude in comparison to pure water [167]. These findings can further advance the use of gold nanoparticles and their assemblies for applications, such as gold mediated transfection and optoporation [10, 11, 32, 36], nanoparticle enhanced laser induced breakdown spectroscopy [19, 20], cell nanosurgery [22], drug release [24, 169], fabrication of functional gold-antibody nanoconjugates [170] and imaging [23].

\subsection{Wavelength dependence of LIOB}

This study, demonstrated that the wavelength dependence for the nanoparticle-mediated optical breakdown threshold for picosecond pulses is dictated by the optical properties of the gold nanoparticle. This is called the absorption regime, where free electron generation is dominated by PTE and absorption cross-section of the nanoparticle will control the optical breakdown lowering. During femtosecond pulses the wavelength dependence for the nanoparticle-mediated optical breakdown threshold is dictated by transitions in the MPI order and the ratio of the maximum electric field outside to the maximum electric field inside the nanoparticle. When this ratio reaches 7 the free electron plasma generation transitions from the absorption dominated regime to the near-field dominated regime. This ratio can be easily calculated from the electromagnetic theory, and will help to 
better interpret LIOB experimental findings and in designing nanoparticles for LIOB applications.

\subsection{Key contributions}

Several key contributions were made to the field of laser-nanoparticle interaction and optical breakdown:

1. The optical behaviour of a single gold nanoparticle on a substrate was analyzed. The evidence of a high sensitivity of the optical properties of a single uncoated nanoparticle was presented. Behaviour of a coated nanoparticle on a substrate proved to be immune to the changes in the local dielectric environment of a particle for the studied silica shell thickness.

2. In this thesis a complete model of the gold nanoparticle-mediated optical breakdown process in water was developed. The overall completeness of the model can be viewed in Table 5.1. As it can be seen from the table, the only area where the current model needs improving is an inclusion of the hydrodynamic effects, which are important during nanosecond pulses, where the expansion starts during the laser pulse and influences the laser-plasma coupling [59].

3. The model was used to analyze the role of gold nanoparticle morphology in optical breakdown phenomena and found that the optical breakdown threshold is highly dependent on the near-field enhancement during picosecond pulses.

4. The optical breakdown model predicts the existence of optical breakdown absorption and near-field regimes, that are based on the ratio of the maximum electric field outside to the maximum electric field inside the nanoparticle. The prediction of these regimes, is an important knowledge for the design of appropriate nanostructures for lowering the optical breakdown threshold. 


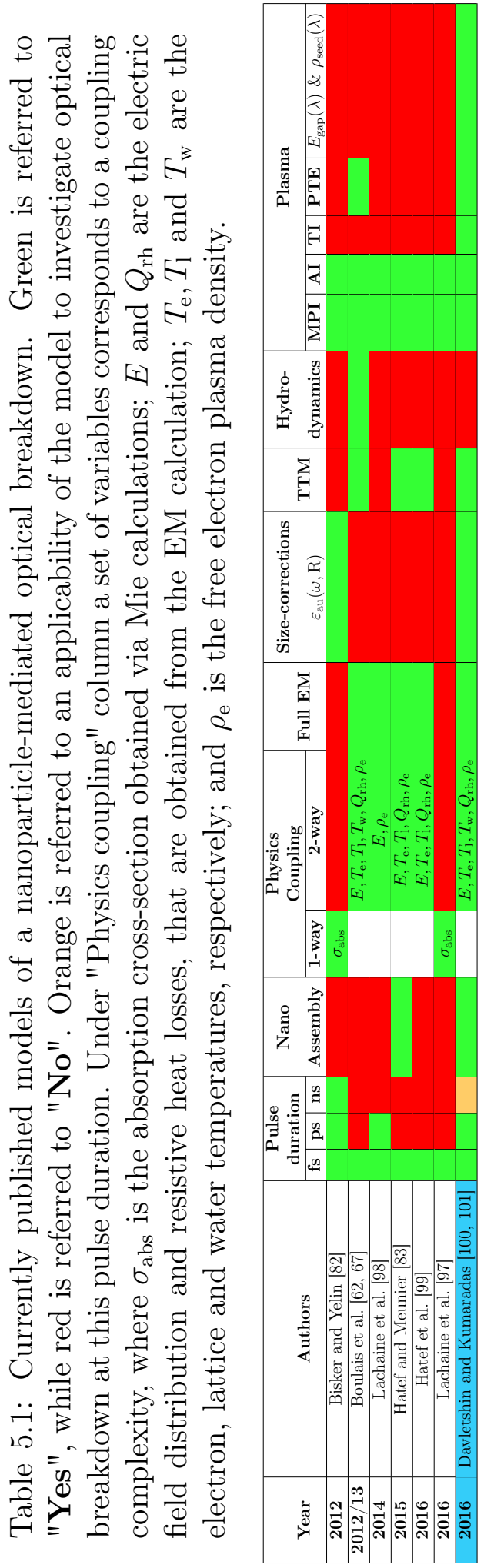





\section{Chapter 6}

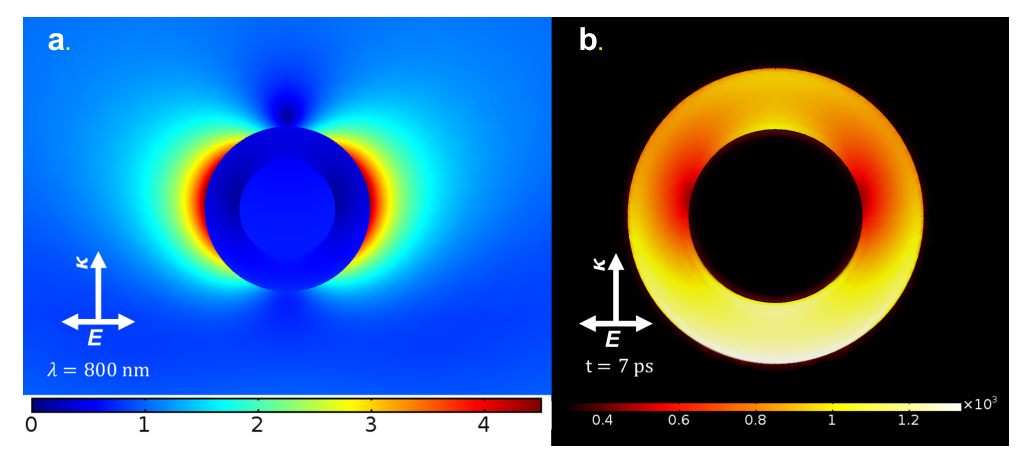

\section{Future work}

The criteria for bubble formation in nanoparticle-mediated optical breakdown was explored to understand the disagreement between optical breakdown threshold predicted by the model for NS660 nanoshell and the measured threshold (see Figure 4.5). This was done by comparing the computed maximum free electron plasma density and temperature in the vicinity of the gold nanoshell against the bubble formation threshold and optoporation efficiency reported by Lachaine et al. [97].

\subsection{A new criterion for optical breakdown}

The laser irradiance threshold needed for optical breakdown and bubble formation, has been historically determined in models as the irradiance that produces a critical free electron density, $\rho_{\text {crit }}$. It's value has been assumed to be between $\rho_{\text {crit }}=10^{18} \mathrm{~cm}^{-3}$ and $\rho_{\text {crit }}=10^{21} \mathrm{~cm}^{-3}$. Additionally, it is possible to calculate the temperature rise due to free electron thermalization and use it as a second criterion for optical breakdown and bubble formation.

From the literature review one can find a wide range of free electron plasma densities, $10^{18}-10^{21} \mathrm{~cm}^{-3}$ [5, 27, 53, 57], that were identified as a parameter that corresponds to the bubble formation and the optical breakdown events. The difference in the three orders of magnitude in the free electron plasma density coming from the differences in the plasma density calculation and comparison against experimental data. In the Section 4.1.3. $\rho_{\mathrm{bf}}=1.8 \times 10^{20} \mathrm{~cm}^{-3}$ [75] was used as a plasma density that is associated 
with the optical breakdown for validation of the model. In the case of NS800, this free electron density $\left(\rho_{\mathrm{bf}}\right)$ worked well and the calculated optical breakdown threshold coincide very well with the experimental fluence threshold of a bubble formation. On the other hand, when the same free electron density was applied to determination of the optical breakdown threshold in the case of NS660 nanoparticle, LIOB model predicted that the optical breakdown event should appear already around $12-15 \mathrm{~mJ} / \mathrm{cm}^{2}$ in comparison to the fluence of $35 \mathrm{~mJ} / \mathrm{cm}^{2}$, where experimentally the bubble formation was detected. Although this disagreement in the calculated and experimentally obtained bubble formation thresholds may be due to the limitations of the experimental setup (which is partially indirectly confirmed by the successful optoporation experiments at $20 \mathrm{~mJ} / \mathrm{cm}^{2}$ [97, Figure 5.1). The free electron density fluence threshold for bubble formation was compared against temperature threshold of bubble formation. This was done by plotting the maximum temperature, $T_{\max }$, of water reached due to free electron thermalization in the vicinity of the gold nanoshell versus the maximum free electron density, $\rho_{\mathrm{e}, \max }$, at the last time step of the optical breakdown modelling in the vicinity of NS660 and NS800 gold nanoshells for different fluences (see Figure 6.1). It should be noted that not all FE solutions reached the end of the $70 \mathrm{fs}$ pulse and some stopped before that.

In the Figure 6.1 black and red dashed lines represents bubble formation thresholds due to temperature, $T_{\mathrm{bf}}=440.7 \mathrm{~K}$, and free electron density of the bubble formation, $\left(\rho_{\mathrm{bf}}=1.8 \times 10^{20} \mathrm{~cm}^{-3}\right)$, respectively that were recently defined by Linz et al. [75] for a pure water without gold nanoparticles. As it can be seen from the Figure 6.1, the intersection of both bubble formation threshold lines for pure water lays outside of the exponential best fit line that combines all data points for both nanoshells and is given by $T_{\max }=300 \exp \left(7 \times 10^{-22} \rho_{\mathrm{e}, \max }\right)$ (blue line, Figure 6.1) with $R^{2}$ value of 0.943 . In order to meet both conditions specified by the temperature of the bubble formation threshold and the lines of best fit, one will need to redefine the bubble formation threshold due to free electron plasma formation to $\rho_{\mathrm{e}}=6 \times 10^{20} \mathrm{~cm}^{-3}$ (see grey dashed line in Figure 6.1). Thus, the bubble formation condition can be redefined as a water heated to the temperature of $440.7 \mathrm{~K}$ and/or a water where free electron plasma generate to the densities above $6 \times 10^{20} \mathrm{~cm}^{-3}$ (which is an average of two nanoshell cases). Furthermore, the fluences of above $11 \mathrm{~mJ} / \mathrm{cm}^{2}$ and $20 \mathrm{~mJ} / \mathrm{cm}^{2}$ for NS800 and NS660 are obtained when the modified plasma density threshold $\left(\rho_{\mathrm{e}}=6 \times 10^{20} \mathrm{~cm}^{-3}\right)$ is used to obtain irradiance optical breakdown threshold from the computational model, respectively. These fluences 


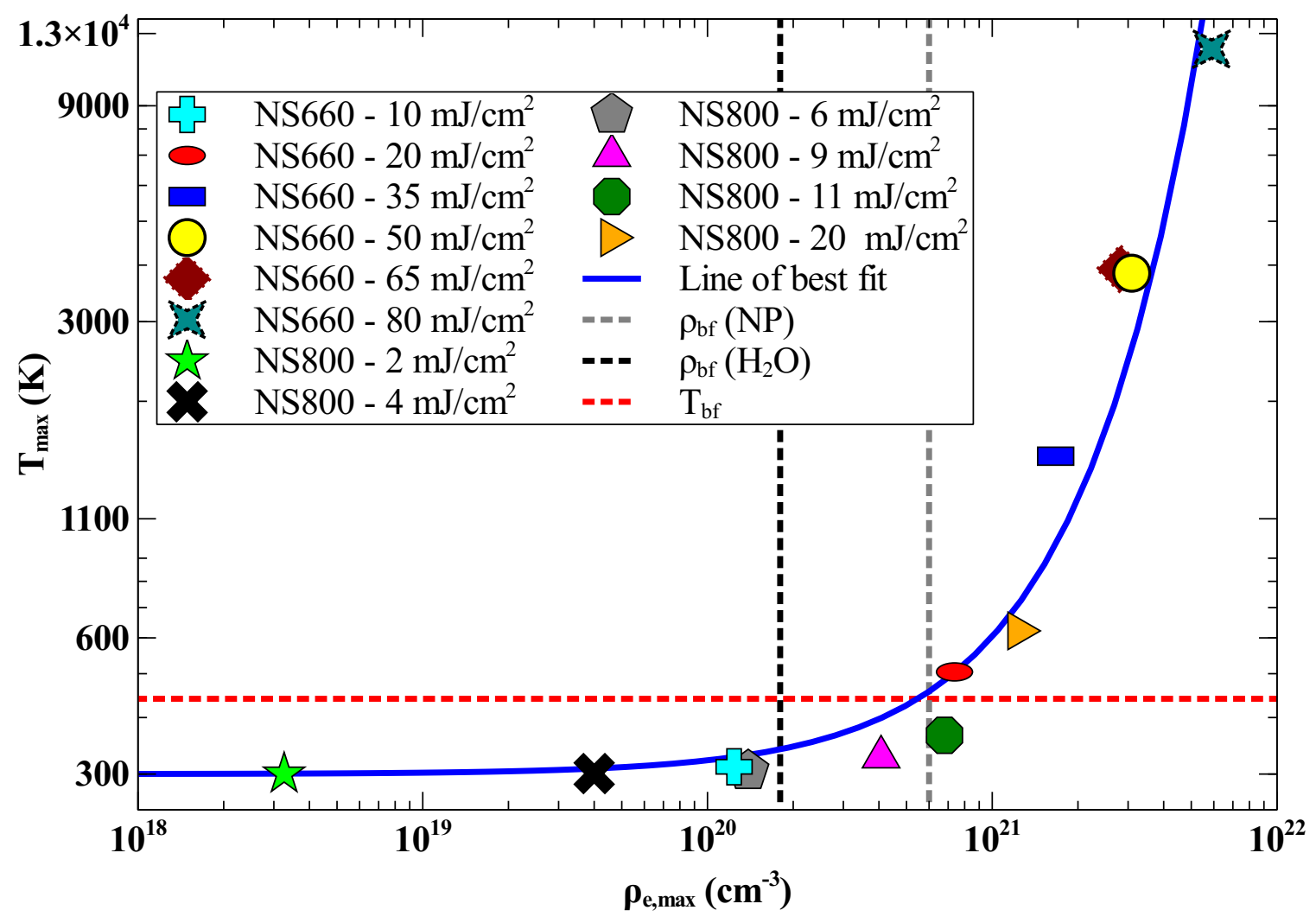

Figure 6.1: Calculated maximum temperature, $T_{\max }$, in the vicinity of the gold nanoshell (NS660 and NS800) at the end of the 70 fs pulse or when the numerical model stopped versus maximum free electron plasma density, $\rho_{\mathrm{e}, \max }$, for a given laser fluence. Black dashed line presents the bubble formation threshold due to free electron density generated, $\rho_{\mathrm{bf}}\left(\mathrm{H}_{2} \mathrm{O}\right),\left(1.8 \times 10^{20} \mathrm{~cm}^{-3}\right)$, while red dashed line is a threshold of bubble formation due to the temperature, $\mathrm{T}_{\mathrm{bf}},(440.7 \mathrm{~K})$ reached in the pure water [75]. The blue line shows an exponential line of best fit, $T_{\max }=$ $300 \exp \left(7 \times 10^{-22} \rho_{\mathrm{e}, \max }\right)$ with $R^{2}=0.943$ for all data points. The grey dashed line represents a new free electron density threshold for bubble formation generated in the vicinity of a nanoparticle, $\rho_{\mathrm{bf}}(\mathrm{NP})$.

are in a good agreement against the optoporation results of Lachaine et al. [97, were a significant optoporation efficiency of live cells was achieved at similar fluences.

Therefore, modelled maximum plasma temperature and the maximum free electron density in the vicinity of gold nanoshells during single $70 \mathrm{fs}$ pulse exposure and the comparison against bubble formation thresholds in temperature $(440.7 \mathrm{~K})$ and free electron 
density $\left(1.8 \times 10^{20} \mathrm{~cm}^{-3}\right)$ and experimental data, provided us with the corrected free electron density threshold of a femtosecond nanoparticle-mediated optical breakdown, $\rho_{\mathrm{e}}=6 \times 10^{20} \mathrm{~cm}^{-3}$. The optical breakdown threshold that is based on newly defined $\rho_{\mathrm{bf}}$ is in very good agreement for both NS660 and NS800 particles against experiments of bubble detection and cell perforation (see Figure 5.1).

\subsection{Sequence of pulses LIOB modelling}

In this research, free electron plasma generation due to a single laser pulse interaction with a nanoparticle was studied. This computational work in the future needs to be extended to plasma generation by sequences of laser pulses. In addition the inclusion of hydrodynamics and the accounting for the phase transitions will enable this model to predict the mechanical effects of a bubble formation to the medium. This will bring a very valuable knowledge to the field of optical breakdown. This task can be accomplished without modifications to the core of the developed model but by the addition of hydrodynamics to the model.

\subsection{Combination of morphology and wavelength dependence study of LIOB}

The work on the wavelength dependence of nanoparticle-mediated optical breakdown should be extended to the other types of nanoparticles, with different size/shape/composition in order to define a range of parameters where the findings stays valid.

\subsection{Nanoparticle-mediated optical breakdown during nanosecond pulse exposure}

Another extension to this work will be an analysis of a plasma generation during nanosecond laser pulse exposure of the nanoparticle. The use of nanosecond pulses becomes popular in the applications utilizing LIOB [10, 29, 30] but theoretical understanding of nanoparticle-mediated LIOB is still missing for ns pulses. 


\subsection{Thermal explosion and melting of nanoparticle}

The main objective of this thesis was to develop a theoretical model of gold-nanoparticle mediated optical breakdown phenomena for use in biomedical applications. The goldnanoparticle mediated optical breakdown phenomena in liquids during ultrashort pulse exposures can cause nanoparticle reshaping [171, 172], melting [173], evaporation [174] and thermal explosion [175]. Several theoretical approaches can be adopted in order to extend the current model to account for these effects . Delfour and Itina [176] provided a numerical model of femtosecond laser-induced fragmentation of gold nanoparticles in water. Strasser et al. [177] modelled pulsed laser-induced heating and evaporation of gold nanoparticles. Zavestovskaya et al. [178] theoretically analyzed nanoparticle heating and fragmentation in water and the development of the instability of charged liquid metal drops. Werner et al. [179] accounted for laser-induced nanoparticle's reshaping and Coulomb explosion during femtosecond pulse exposure in his model. Giammanco et al. [180] include photofragmentation of gold nanoparticles in his model and discussed the roles of melting, evaporation, thermionic emission and photo-assisted ionization during

picosecond laser exposures. Komolov et al. [181] studied the damage of a metal spherical nanoparticle by femtosecond laser pulses by modelling electron photoemission and the formation of positively charged nanoparticle followed by ion emission with removal of the excessive positive charge, which modifies particle structure.

\subsection{Temperature dependent optical properties of gold}

In my study the temperature dependence of optical properties of gold was neglected. This simplification is valid for fs pulses, where the gold's lattice heating occurs after the pulse for pulses shorter than thermalization time of $10 \mathrm{ps.} \mathrm{During} \mathrm{ps} \mathrm{pulses,} \mathrm{depending} \mathrm{on} \mathrm{the}$ morphology and the wavelength used, the temperature of the nanoparticle can reach high values (see Figure 4.10). At high temperatures the optical properties of the nanoparticle, such as absorption and scattering, change. This can be seen in the example of a GNR at several uniform temperatures, where the temperature dependent dielectric function of gold [182] was used (see Figure 4.19). The near-field enhancement at the $860 \mathrm{~nm}$ plasmon resonance wavelength of the GNR is decreases from its maximum $\left(E / E_{\mathrm{inc}}=27.8\right)$ at $300 \mathrm{~K}$ to its minimum $\left(E / E_{\text {inc }}=2.95\right)$ at $1200 \mathrm{~K}$. Since near-field enhancement is an 
important parameter for optimization of gold-nanoparticle mediated optical breakdown, the temperature dependence of the optical properties of gold should be accounted for during the modelling of ps and ns nanoparticle-mediated optical breakdown. This will be done in future studies.

\subsection{Thermionic emission and charging of the nanopar- ticle}

The PTE of electrons off the surface of a nanoparticle positively charges the nanoparticle and creates charged double layer near the surface of nanoparticle. This phenomenon may lower the PTE rate and should be studied for in the future [61, 181, 183]. 


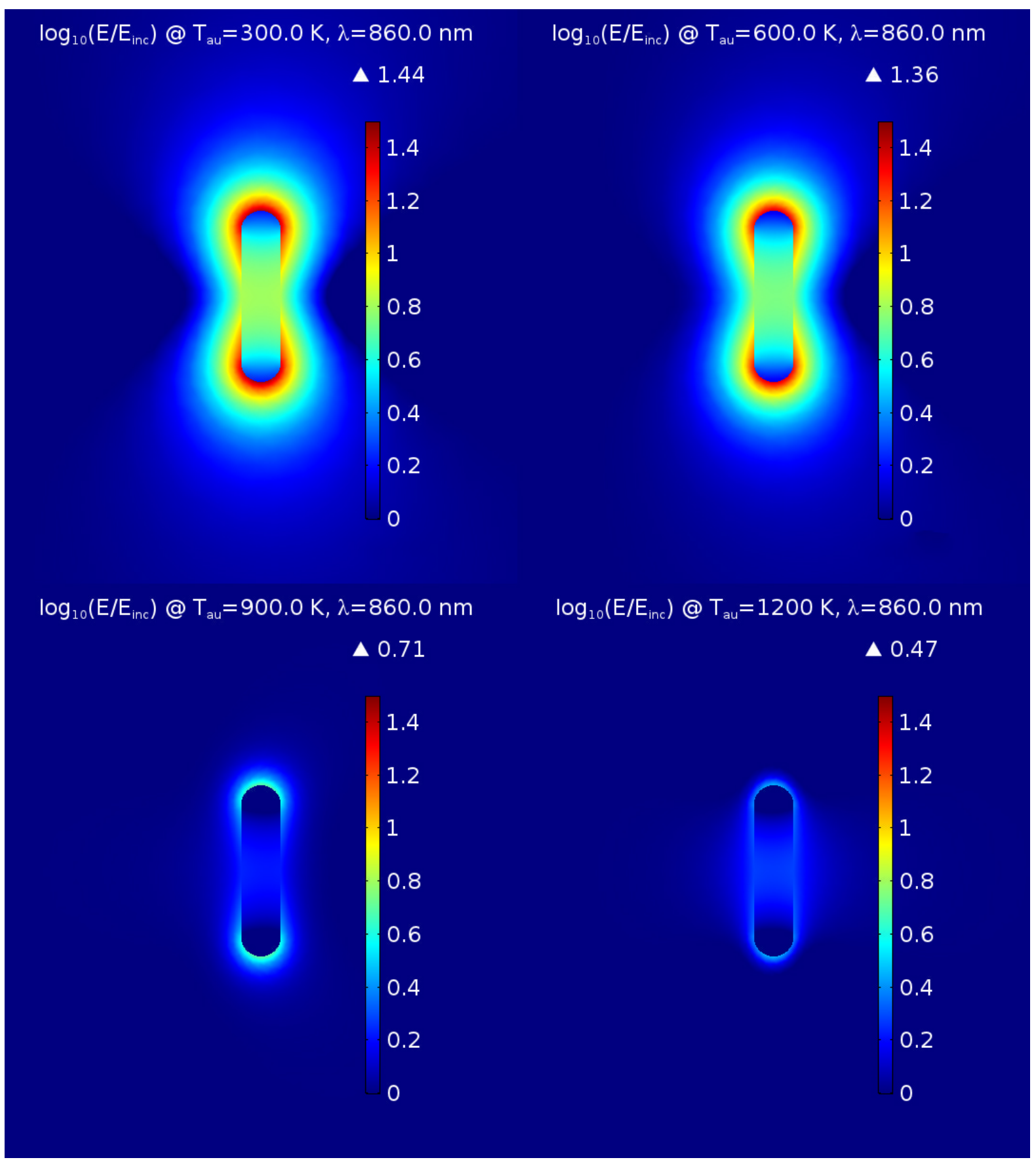

Figure 6.2: Dependence of near-field enhancement, $E / E_{\text {inc }}$, in the vicinity of gold nanorod with uniform temperature of GNR $\left(T_{\mathrm{au}}\right)$ at plasmon resonance wavelength, $\lambda=860 \mathrm{~nm}$. 



\section{References}

[1] R.G. Meyerand and A.F. Haught. Gas breakdown at optical frequencies. Physical Review Letters, 11:401-403, Nov 1963. doi: http://dx.doi.org/10.1103/ PhysRevLett.11.401.

[2] R.W. Minck. Optical frequency electrical discharges in gases. Journal of Applied Physics, 35(1):252-254, 1964. doi: http://dx.doi.org/10.1063/1.1713085.

[3] J.A. Howe. Laser induced breakdown in chlorine. Journal of Applied Physics, 36 (10):3363-3363, 1965. doi: http://dx.doi.org/10.1063/1.1702992.

[4] C. DeMichelis. Laser induced gas breakdown: A bibliographical review. IEEE Journal of Quantum Electronics, 5(4):188-202, Apr 1969. doi: http://dx.doi.org/ 10.1109/JQE.1969.1075758.

[5] N. Bloembergen. Laser-induced electric breakdown in solids. IEEE Journal of Quantum Electronics, 10(3):375-386, Mar 1974. doi: http://dx.doi.org/10.1109/ JQE.1974.1068132.

[6] A.P.M. Michel. Review: Applications of single-shot laser-induced breakdown spectroscopy. Spectrochimica Acta Part B: Atomic Spectroscopy, 65(3):185-191, Mar 2010. doi: http://dx.doi.org/10.1016/j.sab.2010.01.006.

[7] B. C. Stuart, M. D. Feit, A. M. Rubenchik, B. W. Shore, and M. D. Perry. Laserinduced damage in dielectrics with nanosecond to subpicosecond pulses. Physical Review Letters, 74:2248-2251, Mar 1995. doi: http://dx.doi.org/10.1103/ PhysRevLett.74.2248. 
[8] D. Du, X. Liu, G. Korn, J. Squier, and G. Mourou. Laser induced breakdown by impact ionization in sio2 with pulse widths from 7 ns to 150 fs. Applied Physics Letters, 64(23):3071-3073, 1994. doi: http://dx.doi.org/10.1063/1.111350.

[9] A. Vogel, J. Noack, G. Mittmann, and G. Paltauf. Femtosecond-laser-produced lowdensity plasmas in transparent biological media: a tool for the creation of chemical, thermal, and thermomechanical effects below the optical breakdown threshold. volume 4633, pages 23-37, 2002. doi: http://dx.doi.org/10.1117/12.461385.

[10] Y. Arita, M. Ploschner, M. Antkowiak, F. Gunn-Moore, and K. Dholakia. Laserinduced breakdown of an optically trapped gold nanoparticle for single cell transfection. Optics Letters, 38(17):3402-3405, Sep 2013. doi: http://dx.doi.org/10. 1364/OL.38.003402.

[11] J. Baumgart, L. Humbert, É. Boulais, R. Lachaine, J.J. Lebrun, and M. Meunier. Off-resonance plasmonic enhanced femtosecond laser optoporation and transfection of cancer cells. Biomaterials, 33(7):2345 - 2350, 2012. doi: http://dx.doi.org/10. 1016/j.biomaterials.2011.11.062.

[12] I.H. El-Sayed, X. Huang, and M.A. El-Sayed. Selective laser photo-thermal therapy of epithelial carcinoma using anti-egfr antibody conjugated gold nanoparticles. Cancer Letters, 239(1):129 - 135, 2006. doi: http://dx.doi.org/10.1016/j.canlet. 2005.07.035.

[13] X. Huang, P. K. Jain, I.H. El-Sayed, and M.A. El-Sayed. Plasmonic photothermal therapy (pptt) using gold nanoparticles. Lasers in Medical Science, 23(3):217-228, 2007. doi: http://dx.doi.org/10.1007/s10103-007-0470-x.

[14] R.J. Stafford, A. Shetty, A.M. Elliott, J.A. Schwartz, G.P. Goodrich, and J.D. Hazle. Mr temperature imaging of nanoshell mediated laser ablation. International Journal of Hyperthermia, 27(8):782-790, 2011. doi: http://dx.doi.org/10.3109/ 02656736.2011 .614671$.

[15] R.R. Letfullin and T.F. George. Plasmonic Nanomaterials for Nanomedicine, volume Part G of Springer Handbook of Nanomaterials. Springer, Berlin, Heidelberg, 2013. doi: http://dx.doi.org/10.1007/978-3-642-20595-8_30. 
[16] A. Agarwal, S. W. Huang, M. OâĂŹDonnell, K. C. Day, M. Day, N. Kotov, and S. Ashkenazi. Targeted gold nanorod contrast agent for prostate cancer detection by photoacoustic imaging. Journal of Applied Physics, 102(6):064701, 2007. doi: http://dx.doi.org/10.1063/1.2777127.

[17] S. Mallidi, P.P. Joshi, K. Sokolov, and S. Emelianov. On sensitivity of molecular specific photoacoustic imaging using plasmonic gold nanoparticles. pages 63386340, Sept 2009. doi: http://dx.doi.org/10.1109/IEMBS.2009.5333161.

[18] D.R. McCormack, K. Bhattacharyya, R. Kannan, K. Katti, and J.A. Viator. Enhanced photoacoustic detection of melanoma cells using gold nanoparticles. Lasers in Surgery and Medicine, 43(4):333-338, 2011. doi: http://dx.doi.org/10.1002/lsm. 21060.

[19] A. De Giacomo, R. Gaudiuso, C. Koral, M. Dell'Aglio, and O. De Pascale. Nanoparticle-enhanced laser-induced breakdown spectroscopy of metallic samples. Analytical Chemistry, 85(21):10180-10187, 2013. doi: http://dx.doi.org/10.1021/ ac4016165.

[20] A. De Giacomo, R. Gaudiuso, C. Koral, M. Dell'Aglio, and O. De Pascale. Nanoparticle enhanced laser induced breakdown spectroscopy: Effect of nanoparticles deposited on sample surface on laser ablation and plasma emission. Spectrochim. Acta Part B At. Spectrosc., 98:19-27, Aug 2014. doi: http://dx.doi.org/10.1016/j.sab. 2014.05.010.

[21] G. Hüttmann, B. Radt, J. Serbin, B.I. Lange, and R. Birngruber. High Precision Cell Surgery with Nanoparticles? Medical Laser Application, 17(1):9-14, 2002. doi: http://dx.doi.org/10.1078/1615-1615-00039.

[22] É. Boulais, R. Lachaine, A. Hatef, and M. Meunier. Plasmonics for pulsed-laser cell nanosurgery: Fundamentals and applications. Journal of Photochemistry and Photobiology C: Photochemistry Reviews, 17:26-49, Dec 2013. doi: http://dx.doi. org/10.1016/j.jphotochemrev.2013.06.001.

[23] E.Y. Lukianova-Hleb and D.O. Lapotko. Experimental techniques for imaging and 
measuring transient vapor nanobubbles. Applied Physics Letters, 101(26):264102, 2012. doi: http://dx.doi.org/10.1063/1.4772958.

[24] E.Y. Lukianova-Hleb, A. Belyanin, S. Kashinath, X. Wu, and D.O. Lapotko. Plasmonic nanobubble-enhanced endosomal escape processes for selective and guided intracellular delivery of chemotherapy to drug-resistant cancer cells. Biomaterials, 33(6):1821 - 1826, 2012. doi: http://dx.doi.org/10.1016/j.biomaterials.2011.11.015.

[25] S. Kumar, N. Harrison, R. Richards-Kortum, and K. Sokolov. Plasmonic Nanosensors for Imaging Intracellular Biomarkers in Live Cells. Nano Letters, 7(5):13381343, 2007. doi: http://dx.doi.org/10.1021/nl070365i.

[26] Y.R. Davletshin, A. Lombardi, M.F. Cardinal, V. Juvé, A. Crut, P. Maioli, L.M. Liz-Marzán, F. Vallée, and J.C. Del Fatti, N.and Kumaradas. A quantitative study of the environmental effects on the optical response of gold nanorods. ACS Nano, 6(9):8183-8193, Sep 2012. doi: http://dx.doi.org/10.1021/nn302869v.

[27] A. Vogel, J. Noack, G. Hüttman, and G. Paltauf. Mechanisms of femtosecond laser nanosurgery of cells and tissues. Applied Physics B, 81(8):1015-1047, 2005. doi: http://dx.doi.org/10.1007/s00340-005-2036-6.

[28] R.R. Letfullin, T.F. George, G.C. Duree, and B.M. Bollinger. Ultrashort laser pulse heating of nanoparticles: Comparison of theoretical approaches. Advances in Optical Technologies, 2008, 2008. doi: http://dx.doi.org/10.1155/2008/251718.

[29] B.S-L. Lalonde, É. Boulais, J.J. Lebrun, and M. Meunier. Visible and near infrared resonance plasmonic enhanced nanosecond laser optoporation of cancer cells. Biomedical Optics Express, 4(4):490-499, Apr 2013. doi: http://dx.doi.org/10. 1364/BOE.4.000490.

[30] J. Krawinkel, M.L. Torres-Mapa, K. Werelius, A. Heisterkamp, S. Rüttermann, G.E. Romanos, and S. Gerhardt-Szép. Gold nanoparticle-mediated delivery of molecules into primary human gingival fibroblasts using ns-laser pulses: A pilot study. Materials, 9(5):397, 2016. doi: http://dx.doi.org/10.3390/ma9050397.

[31] S. Kalies, L. Gentemann, M. Schomaker, D. Heinemann, T. Ripken, and H. Meyer. Surface modification of silica particles with gold nanoparticles as an augmentation 
of gold nanoparticle mediated laser perforation. Biomedical Optics Express, 5(8): 2686-2696, Aug 2014. doi: http://dx.doi.org/10.1364/BOE.5.002686.

[32] D. Heinemann, M. Schomaker, S. Kalies, M. Schieck, R. Carlson, H. Murua Escobar, T. Ripken, H. Meyer, and A. Heisterkamp. Gold Nanoparticle Mediated Laser Transfection for Efficient siRNA Mediated Gene Knock Down. PLoS One, 8(3): e58604, 2013. doi: http://dx.doi.org/10.1371/journal.pone.0058604.

[33] D. Heinemann, S. Kalies, M. Schomaker, W. Ertmer, H. Murua Escobar, H. Meyer, and T. Ripken. Delivery of proteins to mammalian cells via gold nanoparticle mediated laser transfection. Nanotechnology, 25(24):245101, 2014. doi: http://dx. doi.org/10.1088/0957-4484/25/24/245101.

[34] M. Schomaker, H. Fehlauer, W. Bintig, A. Ngezahayo, I. Nolte, H. Murua Escobar, H. Lubatschowski, and A. Heisterkamp. Fs-laser cell perforation using gold nanoparticles of different shapes. Proc. SPIE, 7589:75890C-75890C-5, 2010. doi: http://dx.doi.org/10.1117/12.842446.

[35] M. Schomaker, D. Killian, S. Willenbrock, D. Heinemann, S. Kalies, A. Ngezahayo, I. Nolte, T. Ripken, C. Junghanß, H. Meyer, H. Murua Escobar, and A. Heisterkamp. Biophysical effects in off-resonant gold nanoparticle mediated (GNOME) laser transfection of cell lines, primary- and stem cells using fs laser pulses. Journal of Biophotonics, 8(8):646-658, 2015. doi: http://dx.doi.org/10. 1002/jbio.201400065.

[36] M. Schomaker, D. Heinemann, S. Kalies, S. Willenbrock, S. Wagner, I. Nolte, T. Ripken, H. Murua Escobar, H. Meyer, and A. Heisterkamp. Characterization of nanoparticle mediated laser transfection by femtosecond laser pulses for applications in molecular medicine. Journal of Nanobiotechnology, 13(1):10, 2015. doi: http://dx.doi.org/10.1186/s12951-014-0057-1.

[37] K.L. Kelly, E. Coronado, L.L. Zhao, and G.C. Schatz. The Optical Properties of Metal Nanoparticles: The Influence of Size, Shape, and Dielectric Environment. The Journal of Physical Chemistry B, 107(3):668-677, 2003. doi: http://dx.doi. org/10.1021/jp026731y. 
[38] C. Noguez. Surface plasmons on metal nanoparticles:âĂL' the influence of shape and physical environment. The Journal of Physical Chemistry C, 111(10):3806-3819, 2007. doi: http://dx.doi.org/10.1021/jp066539m.

[39] H. Chen, X. Kou, Z. Yang, W. NI, and J. Wang. Shape- and size-dependent refractive index sensitivity of gold nanoparticles. Langmuir, 24(10):5233-5237, 2008. doi: http://dx.doi.org/10.1021/la800305j.

[40] A. Lombardi, M. Loumaigne, A. Crut, P. Maioli, N. Del Fatti, F. Vallée, M. SpuchCalvar, J. Burgin, J. Majimel, and M. Tréguer-Delapierre. Surface plasmon resonance properties of single elongated nano-objects: gold nanobipyramids and nanorods. Langmuir, 28(24):9027-33, 2012. doi: http://dx.doi.org/10.1021/ la300210h.

[41] W. Rechberger, A. Hohenau, A. Leitner, J.R. Krenn, B. Lamprecht, and F.R. Aussenegg. Optical properties of two interacting gold nanoparticles. Optics Communications, 220(1-3):137 - 141, 2003. doi: http://dx.doi.org/10.1016/ S0030-4018(03)01357-9.

[42] G.V. Hartland. Optical Studies of Dynamics in Noble Metal Nanostructures. Chemical Reviews, 111(6):3858-3887, Jun 2011. doi: http://dx.doi.org/10.1021/ cr1002547.

[43] A. Crut, P. Maioli, N. Del Fatti, and F. Vallée. Optical absorption and scattering spectroscopies of single nano-objects. Chemical Society Reviews, 43:3921-3956, 2014. doi: http://dx.doi.org/10.1039/C3CS60367A.

[44] U. Kreibig and M. Vollmer. Optical Properties of Metal Clusters, volume 25 of Springer Series in Materials Science. Springer Berlin Heidelberg, Berlin, Heidelberg, 1995.

[45] M. Dell'Aglio, R. Gaudiuso, O. De Pascale, and A. De Giacomo. Mechanisms and processes of pulsed laser ablation in liquids during nanoparticle production. Applied Surface Science, 348:4-9, 2015. doi: http://dx.doi.org/10.1016/j.apsusc. 2015.01.082. 
[46] C. Sönnichsen, T. Franzl, T. Wilk, G. von Plessen, and J. Feldmann. Drastic reduction of plasmon damping in gold nanorods. Physical Review Letters, 88:077402, Jan 2002. doi: http://dx.doi.org/10.1103/PhysRevLett.88.077402.

[47] M.A. van Dijk, A.L. Tchebotareva, M. Orrit, M. Lippitz, S. Berciaud, D. Lasne, L. Cognet, and B. Lounis. Absorption and scattering microscopy of single metal nanoparticles. Physical Chemistry Chemical Physics, 8:3486-3495, 2006. doi: http: //dx.doi.org/10.1039/B606090K.

[48] P.K. Jain, X. Huang, I. H El-Sayed, and M.A. El-Sayed. Noble Metals on the Nanoscale: Optical and Photothermal Properties and Some Applications in Imaging, Sensing, Biology, and Medicine. Accounts of Chemical Research, 41(12):15781586, Dec 2008. doi: http://dx.doi.org/10.1021/ar7002804.

[49] M. Hu, J. Chen, Z.-Y. Li, L. Au, G.V. Hartland, X. Li, M. Marquez, and Y. Xia. Gold nanostructures: engineering their plasmonic properties for biomedical applications. Chemical Society Reviews, 35:1084-1094, 2006. doi: http: //dx.doi.org/10.1039/B517615H.

[50] X. Huang, I.H. El-Sayed, W. Qian, and M.A. El-Sayed. Cancer Cells Assemble and Align Gold Nanorods Conjugated to Antibodies to Produce Highly Enhanced, Sharp, and Polarized Surface Raman Spectra: a Potential Cancer Diagnostic Marker. Nano Letters, 7(6):1591-1597, Jun 2007. doi: http://dx.doi.org/10. 1021/nl070472c.

[51] J. Becker, A. Trügler, A. Jakab, U. Hohenester, and C. Sönnichsen. The optimal aspect ratio of gold nanorods for plasmonic bio-sensing. Plasmonics, 5:161-167, 2010. doi: http://dx.doi.org/10.1007/s11468-010-9130-2.

[52] Z. Li, W. Mao, M.s. Devadas, and G.V. Hartland. Absorption spectroscopy of single optically trapped gold nanorods. Nano Letters, 15(11):7731-7735, 2015. doi: http://dx.doi.org/10.1021/acs.nanolett.5b03833.

[53] C.A. Sacchi. Laser-induced electric breakdown in water. JOURNAL OF THE OPTICAL SOCIETY OF AMERICA B-OPTICAL PHYSICS, 8(2):337-345, Feb 1991. doi: http://dx.doi.org/10.1364/JOSAB.8.000337. 
[54] P.K. Kennedy. First-order model for computation of laser-induced breakdown thresholds in ocular and aqueous media: Part I - theory. IEEE Journal of Quantum Electronics, 31(12):2241-2249, Dec 1995. doi: http://dx.doi.org/10.1109/3.477753.

[55] A. Vogel, K. Nahen, D. Theisen, and J. Noack. Plasma formation in water by picosecond and nanosecond Nd:YAG laser pulses. I. Optical breakdown at threshold and superthreshold irradiance. IEEE Journal of Quantum Electronics, 2(4):847860, Dec 1996. doi: http://dx.doi.org/10.1109/2944.577307.

[56] Q. Feng, J.V. Moloney, A.C. Newell, E.M. Wright, K. Cook, P.K. Kennedy, D.X. Hammer, B.A. Rockwell, and C.R. Thompson. Theory and simulation on the threshold of water breakdown induced by focused ultrashort laser pulses. IEEE Journal of Quantum Electronics, 33(2):127-137, Feb 1997. doi: http://dx.doi.org/ $10.1109 / 3.552252$.

[57] J. Noack and A. Vogel. Laser-induced plasma formation in water at nanosecond to femtosecond time scales: calculation of thresholds, absorption coefficients, and energy density. IEEE Journal of Quantum Electronics, 35(8):1156-1167, aug 1999. doi: http://dx.doi.org/10.1109/3.777215.

[58] P.K. Kennedy, S.A. Boppart, D.X. Hammer, B.A. Rockwell, G.D. Noojin, and W.P. Roach. A first order model for computation of laser-induced breakdown thresholds in ocular and aqueous media-Part II: Comparison to experiment. IEEE Journal of Quantum Electronics, 31(12):2250 - 2257, Dec 1995. doi: http://dx.doi.org/10. $1109 / 3.477754$.

[59] N. Linz, S. Freidank, X. Liang, J. Noack, G. Paltauf, and A. Vogel. Roles of tunneling, multiphoton ionization, and cascade ionization for optical breakdown in aqueous media. AFOSR Int. Res. Initiat. Proj. SPC 053010 / EOARD, (Grant FA 8655-05-1-3010):0-206, 2009. URL http://www.dtic.mil/dtic/tr/fulltext/ u2/a521817.pdf.

[60] B. Varghese, V. Bonito, M. Jurna, J. Palero, and Margaret H.R. V. Influence of absorption induced thermal initiation pathway on irradiance threshold for laser induced breakdown. Biomedical Optics Express, 6(4):1234, Apr 2015. doi: http: //dx.doi.org/10.1364/BOE.6.001234. 
[61] N.M. Bulgakova, R. Stoian, A. Rosenfeld, I.V. Hertel, and E.E.B. Campbell. Electronic transport and consequences for material removal in ultrafast pulsed laser ablation of materials. Phys. Rev. B, 69(5):054102, feb 2004. doi: http: //dx.doi.org/10.1103/PhysRevB.69.054102.

[62] É. Boulais, R. Lachaine, and M. Meunier. Plasma Mediated off-Resonance Plasmonic Enhanced Ultrafast Laser-Induced Nanocavitation. Nano Letters, 12(9): 4763-4769, 2012. doi: http://dx.doi.org/10.1021/nl302200w.

[63] A. Kaiser, B. Rethfeld, M. Vicanek, and G. Simon. Microscopic processes in dielectrics under irradiation by subpicosecond laser pulses. Physical Review B, 61: 11437-11450, May 2000. doi: http://dx.doi.org/10.1103/PhysRevB.61.11437.

[64] A. Vogel, S. Busch, and U. Parlitz. Shock wave emission and cavitation bubble generation by picosecond and nanosecond optical breakdown in water. The Journal of the Acoustical Society of America, 100(1):148-165, 1996. doi: http://dx.doi.org/ $10.1121 / 1.415878$.

[65] D.X. Hammer, R.J. Thomas, G.D. Noojin, B.A. Rockwell, P.K. Kennedy, and W.P. Roach. Experimental investigation of ultrashort pulse laser-induced breakdown thresholds in aqueous media. IEEE Journal of Quantum Electronics, 32(4):670678, Apr 1996. doi: http://dx.doi.org/10.1109/3.488842.

[66] X.X. Liang, N. Linz, J. Noack, and A. Vogel. Modelling of optical breakdown in dielectrics including thermal effects relevant for nanosecond pulses and sequences of ultra-short laser pulses. In Lasers and Electro-Optics 2009 and the European Quantum Electronics Conference. CLEO Europe - EQEC 2009. European Conference on, page 1, Jun 2009. doi: http://dx.doi.org/10.1109/CLEOE-EQEC.2009.5191546.

[67] É. Boulais, R. Lachaine, and M. Meunier. Plasma-mediated nanocavitation and photothermal effects in ultrafast laser irradiation of gold nanorods in water. The Journal of Physical Chemistry C, 117(18):9386-9396, 2013. doi: http://dx.doi.org/ 10.1021/jp312475h.

[68] A. Plech, V. Kotaidis, S. Grésillon, C. Dahmen, and G. von Plessen. Laser-induced heating and melting of gold nanoparticles studied by time-resolved x-ray scatter- 
ing. Physical Review B, 70:195423, Nov 2004. doi: http://dx.doi.org/10.1103/ PhysRevB.70.195423.

[69] C.-K. Sun, F. Vallée, L. Acioli, E. P. Ippen, and J. G. Fujimoto. Femtosecond investigation of electron thermalization in gold. Physical Review B, 48:12365-12368, Oct 1993. doi: http://dx.doi.org/10.1103/PhysRevB.48.12365.

[70] S. Link, C. Burda, M.B. Mohamed, B. Nikoobakht, and M.A. El-Sayed. Femtosecond transient-absorption dynamics of colloidal gold nanorods: Shape independence of the electron-phonon relaxation time. Physical Review B, 61(9):6086-6090, Mar 2000. doi: http://dx.doi.org/10.1103/PhysRevB.61.6086.

[71] A. Spiro, M. Lowe, and G. Pasmanik. Drilling rate of five metals with picosecond laser pulses at 355, 532, and $1064 \mathrm{~nm}$. Applied Physics A, 107(4):801-808, 2012.

[72] F. Docchio, C.A. Sacchi, and J. Marshall. Experimental investigation of optical breakdown thresholds in ocular media under single pulse irradiation with different pulse durations. Lasers Ophthalmology, (1):83-93, 1986.

[73] S. Freidank, N. Linz, H. Vogelmann, T. Trickl, K. Werelius, and A. Vogel. Wavelength dependence of nanosecond and femtosecond optical breakdown in water: Experimental investigations. CLEO Europe - EQEC, pages 1-1, June 2009. doi: http://dx.doi.org/10.1109/CLEOE-EQEC.2009.5192286.

[74] N. Linz, S. Freidank, X.-X. Liang, H. Vogelmann, T. Trickl, and A. Vogel. Wavelength dependence of nanosecond infrared laser-induced breakdown in water: Evidence for multiphoton initiation via an intermediate state. Physical Review B, 91: 134114, Apr 2015. doi: http://dx.doi.org/10.1103/PhysRevB.91.134114.

[75] N. Linz, S. Freidank, X.-X. Liang, and A. Vogel. Wavelength dependence of femtosecond laser-induced breakdown in water and implications for laser surgery. Physical Review B, 94:024113, Jul 2016. doi: http://dx.doi.org/10.1103/PhysRevB.94. 024113.

[76] N. Linz, X.X. Liang, J. Noack, and A. Vogel. Wavelength dependence of nanosecond and femtosecond optical breakdown in water: Theoretical analysis. CLEO Europe 
- EQEC, pages 1-1, June 2009. doi: http://dx.doi.org/10.1109/CLEOE-EQEC. 2009.5191756 .

[77] F. Williams, S. P. Varma, and S. Hillenius. Liquid water as a lone pair amorphous semiconductor. Journal of Chemical Physics, 64(4):1549-1554, 1976. doi: http: //dx.doi.org/10.1063/1.432377.

[78] M.U. Sander, M.S. Gudiksen, K. Luther, and J. Troe. Liquid water ionization: mechanistic implications of the $\mathrm{H} / \mathrm{D}$ isotope effect in the geminate recombination of hydrated electrons. Chemical Physics, 258(2-3):257-265, 2000. doi: http://dx. doi.org/10.1016/S0301-0104(00)00170-1.

[79] C.G. Elles, A.E. Jailaubekov, R.A. Crowell, and S.E. Bradforth. Excitation-energy dependence of the mechanism for two-photon ionization of liquid h2o and $\mathrm{d} 2 \mathrm{o}$ from 8.3 to $12.4 \mathrm{ev.} \mathrm{The} \mathrm{Journal} \mathrm{of} \mathrm{Chemical} \mathrm{Physics,} \mathrm{125(4),} \mathrm{2006.} \mathrm{doi:} \mathrm{http:}$ //dx.doi.org/10.1063/1.2217738.

[80] D.M. Bartels and R.A. Crowell. Photoionization yield vs energy in h2o and d2o. The Journal of Physical Chemistry A, 104(15):3349-3355, 2000. doi: http://dx. doi.org/10.1021/jp9941460.

[81] D.H. Son, P. Kambhampati, T.W. Kee, , and P.F. Barbara. Delocalizing electrons in water with light. The Journal of Physical Chemistry A, 105(36):8269-8272, 2001. doi: http://dx.doi.org/10.1021/jp012431p.

[82] G. Bisker and D. Yelin. Noble-metal nanoparticles and short pulses for nanomanipulations: theoretical analysis. Journal of the Optical Society of America B, 29 (6):1383, Jun 2012. doi: http://dx.doi.org/10.1364/JOSAB.29.001383.

[83] A. Hatef and M. Meunier. Plasma-mediated photothermal effects in ultrafast laser irradiation of gold nanoparticle dimers in water. Optics Express, 23(3):1967, Feb 2015. doi: http://dx.doi.org/10.1364/OE.23.001967.

[84] S. Kalies, T. Birr, D. Heinemann, M. Schomaker, T. Ripken, A. Heisterkamp, and H. Meyer. Enhancement of extracellular molecule uptake in plasmonic laser perforation. Journal of Biophotonics, 7(7):474-482, 2014. doi: http://dx.doi.org/ 10.1002/jbio.201200200. 
[85] D. Fava, M.A. Winnik, and E. Kumacheva. Photothermally-triggered self-assembly of gold nanorods. Chemical Communications, (18):2571-2573, 2009. doi: http: //dx.doi.org/10.1039/B901412H.

[86] L. Wang, L. Xu, H. Kuang, C. Xu, and N.A. Kotov. Dynamic Nanoparticle Assemblies. Accounts of Chemical Research, 45(11):1916-1926, 2012. doi: http://dx.doi.org/10.1021/ar200305f.

[87] M. Quinten. Optical constants of gold and silver clusters in the spectral range between $1.5 \mathrm{eV}$ and $4.5 \mathrm{eV}$. Zeitschrift für Physik B Condensed Matter, 101(2): 211-217, 1996. doi: http://dx.doi.org/10.1007/s002570050202.

[88] O.L. Muskens, G. Bachelier, N. Del Fatti, F. Vallée, A. Brioude, X. Jiang, and M.P. Pileni. Quantitative absorption spectroscopy of a single gold nanorod. Journal of Physical Chemistry C, 112(24):8917-8921, June 2008. doi: http://dx.doi.org/ $10.1021 /$ jp 8012865 .

[89] G. Mie. Beiträge zur optik trüber medien, speziell kolloidaler metallösungen. Annalen der Physik, 330(3):377-445, 1908. ISSN 1521-3889. doi: http://dx.doi.org/ 10.1002/andp.19083300302.

[90] P. Billaud, S. Marhaba, E. Cottancin, L. Arnaud, G. Bachelier, C. Bonnet, N. Del Fatti, J. Lermé, F. Vallée, J.L. Vialle, M. Broyer, and M. Pellarin. Correlation between the extinction spectrum of a single metal nanoparticle and its electron microscopy image. Journal of Physical Chemistry C, 112(4):978-982, 2008. doi: http://dx.doi.org/10.1021/jp076955m.

[91] E. Cottancin, G. Celep, J. Lermé, M. Pellarin, J.R. Huntzinger, J.L. Vialle, and M. Broyer. Optical Properties of Noble Metal Clusters as a Function of the Size: Comparison between Experiments and a Semi-Quantal Theory. Theoretical Chemistry Accounts, 116(4-5):514-523, 2006. doi: http://dx.doi.org/10.1007/ s00214-006-0089-1.

[92] C. Novo, D. Gomez, J. Perez-Juste, Z. Zhang, H. Petrova, M. Reismann, P. Mulvaney, and G.V. Hartland. Contributions from Radiation Damping and Surface Scattering to the Linewidth of the Longitudinal Plasmon Band of Gold Nanorods: 
a Single Particle Study. Physical Chemistry Chemical Physics, 8(30):3540-3546, Aug 2006. doi: http://dx.doi.org/10.1039/B604856K.

[93] I. Tsukerman. Computational Methods for Nanoscale Applications. Springer, 2008. doi: http://dx.doi.org/10.1007/978-0-387-74778-1.

[94] J.L. Volakis, A. Chatterjee, and L.C. Kempel. Finite Element Method for Electromagnetics: Antennas, Microwave Circuits, and Scattering Applications. WileyIEEE Press, 1998.

[95] J. Jin. The Finite Element Method in Electromagnetics. Wiley-IEEE Press, 2002.

[96] R.T. Fenner. Finite Element Methods for Engineers. Oxford University Press, 2003.

[97] R. Lachaine, C. Boutopoulos, P-Y. Lajoie, É. Boulais, and M. Meunier. Rational design of plasmonic nanoparticles for enhanced cavitation and cell perforation. Nano Letters, 16(5):3187-3194, 2016. doi: http://dx.doi.org/10.1021/acs.nanolett. 6b00562.

[98] R. Lachaine, É. Boulais, and M. Meunier. From thermo- to plasma-mediated ultrafast laser-induced plasmonic nanobubbles. ACS Photonics, 1(4):331-336, 2014. doi: http://dx.doi.org/10.1021/ph400018s.

[99] A. Hatef, B. Darvish, A. Burke, A. Dagallier, and M. Meunier. Computational characterization of plasma effects in ultrafast laser irradiation of spherical gold nanostructures for photothermal therapy. Journal of Physics D: Applied Physics, 49(10):105401, 2016. doi: http://dx.doi.org/10.1088/0022-3727/49/10/105401.

[100] Y.R. Davletshin and J.C. Kumaradas. The role of morphology and coupling of gold nanoparticles in optical breakdown during picosecond pulse exposures. Beilstein Journal of Nanotechnology, 7:869-880, 2016. doi: http://dx.doi.org/10.3762/ bjnano.7.79.

[101] Y.R. Davletshin and J.C. Kumaradas. The wavelength dependence of gold nanorodmediated optical breakdown during infrared ultrashort pulses. Annalen der Physik, 528, 2016. doi: http://dx.doi.org/10.1002/andp.201600135. 
[102] S. Berciaud, L. Cognet, P. Tamarat, and B. Lounis. Observation of Intrinsic Size Effects in the Optical Response of Individual Gold Nanoparticles. Nano Letters, 5 (3):515-518, Mar 2005. doi: http://dx.doi.org/10.1021/nl050062t.

[103] O.L. Muskens, D. Christofilos, N. Del Fatti, and F. Vallée. Optical response of a single noble metal nanoparticle. Journal of Optics A: Pure and Applied Optics, 8 (4):S264-S272, Apr 2006.

[104] H. Baida, D. Christofilos, P. Maioli, A. Crut, N. Del Fatti, and F. Vallée. Surface plasmon resonance spectroscopy of single surfactant-stabilized gold nanoparticles. European Physical Journal D, 63(2):293-299, Apr 2011. doi: http://dx.doi.org/10. 1140/epjd/e2010-10594-y.

[105] L. Genzel, T.P. Martin, and U. Kreibig. Dielectric function and plasma resonances of small metal particles. Zeitschrift für Physik B Condensed Matter, 21(4):339-346, 1975. doi: http://dx.doi.org/10.1007/BF01325393.

[106] W.A. Kraus and G.C. Schatz. Plasmon resonance broadening in small metal particles. Journal of Chemical Physics, 79:6130-6139, 1983. doi: http://dx.doi.org/ $10.1063 / 1.445794$.

[107] J. Sancho-Parramon. Surface plasmon resonance broadening of metallic particles in the quasi-static approximation: A numerical study of size confinement and interparticle interaction effects. Nanotechnology, 20, 2009. doi: http://dx.doi.org/ 10.1088/0957-4484/20/23/235706.

[108] O.L. Muskens, P. Billaud, M. Broyer, N. Del Fatti, and F. Vallée. Optical extinction spectrum of a single metal nanoparticle: Quantitative characterization of a particle and of its local environment. Physical Review B, 78:205410, Nov 2008. doi: http: //dx.doi.org/10.1103/PhysRevB.78.205410.

[109] M.A. Otte, B. Sepúlveda, W. Ni, J.P. Juste, L.M. Liz-Marzán, and L.M. Lechuga. Identification of the optimal spectral region for plasmonic and nanoplasmonic sensing. ACS Nano, 4(1):349-357, 2010. doi: http://dx.doi.org/10.1021/nn901024e. 
[110] P. Billaud, J.R. Huntzinger, E. Cottancin, J. Lermé, M. Pellarin, L. Arnaud, M. Broyer, N. Del Fatti, and F. Vallée. Optical extinction spectroscopy of single silver nanoparticles. European Physical Journal D, 43(1-3):271-274, 2007. doi: http://dx.doi.org/10.1140/epjd/e2007-00112-y.

[111] C. Novo, A.M. Funston, I. Pastoriza-Santos, L.M. Liz-Marzán, and P. Mulvaney. Influence of the medium refractive index on the optical properties of single gold triangular prisms on a substrate. Journal of Physical Chemistry C, 112(1):3-7, 2008. doi: http://dx.doi.org/10.1021/jp709606u.

[112] V.K. Pustovalov. Theoretical study of heating of spherical nanoparticle in media by short laser pulses. Chemical Physics, 308(1-2):103-108, 2005. doi: http://dx. doi.org/10.1016/j.chemphys.2004.08.005.

[113] Chen, J. K. and Beraun, J. E. Numerical study of ultrashort laser pulse interactions with metal films. Numerical Heat Transfer, Part A: Applications, 40(1):1-20, Jul 2001. doi: http://dx.doi.org/10.1080/104077801300348842.

[114] L.V. Keldysh. Ionization in the field of a strong electromagnetic wave. Jetp, 20(5): 1307-1314, 1965. URL http://www.jetp.ac.ru/cgi-bin/dn/e_020_05_1307. pdf.

[115] P.B. Johnson and R.W. Christy. Optical Constants of the Noble Metals. Physical Review B, 6(12):4370-4379, Dec 1972. doi: http://dx.doi.org/10.1103/PhysRevB. 6.4370 .

[116] S.I. Anisimov, B.L. Kapeliovich, and T.L. Perel'man. Electron emission from metal surfaces exposed to ultrashort laser pulses. Journal of Experimental and Theoretical Physics, 39(2):375, 08 1974. URL http://www.jetp.ac.ru/cgi-bin/e/index/e/ $39 / 2 / \mathrm{p} 375 ? \mathrm{a}=$ list.

[117] COMSOL AB. Electromagnetics Module: User's Guide. COMSOL Multiphysics 5.2, 2015.

[118] O. Ekici, R.K. Harrison, N.J. Durr, D.S. Eversole, M. Lee, and A. Ben-Yakar. Thermal analysis of gold nanorods heated with femtosecond laser pulses. Journal 
of Physics D: Applied Physics, 41(18):185501, 2008. doi: http://dx.doi.org/10. 1088/0022-3727/41/18/185501.

[119] J. Soussi, S. Volz, B. Palpant, and Y. Chalopin. A detailed microscopic study of the heat transfer at a water gold interface coated with a polymer. Applied Physics Letters, 106(9), 2015. doi: http://dx.doi.org/10.1063/1.4913905.

[120] X. Wu, Y. Ni, J. Zhu, N.D. Burrows, C.J. Murphy, T. Dumitrica, and X. Wang. Thermal transport across surfactant layers on gold nanorods in aqueous solution. ACS Applied Materials \& Interfaces, 8(16):10581-10589, 2016. doi: http://dx.doi. org/10.1021/acsami.5b12163.

[121] J.K. Chen, D.Y. Tzou, and J.E. Beraun. A semiclassical two-temperature model for ultrafast laser heating. International Journal of Heat and Mass Transfer, 49 (1-2):307-316, Jan 2006. doi: http://dx.doi.org/10.1016/j.ijheatmasstransfer.2005. 06.022 .

[122] V.P. Zharov, R.R. Letfullin, and E.N. Galitovskaya. Microbubbles-overlapping mode for laser killing of cancer cells with absorbing nanoparticle clusters. Journal of Physics D: Applied Physics, 38(15):2571, 2005. doi: http://dx.doi.org/10.1088/ 0022-3727/38/15/007.

[123] M.V. Ammosov, N.B. Delone, and V.P. Krainov. Tunnel ionization of complex atoms and of atomic ions in an alternating electromagnetic field. Soviet Physics JETP, 64(6):1191-1194, 1986.

[124] D.N. Nikogosyan, A.A. Oraevsky, and V.I. Rupasov. Two-photon ionization and dissociation of liquid water by powerful laser UV radiation. Chemical Physics, 77 (1):131-143, 1983. doi: http://dx.doi.org/10.1016/0301-0104(83)85070-8.

[125] C.G. Morgan. Laser-induced breakdown of gases. Reports on Progress in Physics, 38(5):621-665, 1975. doi: http://dx.doi.org/10.1088/0034-4885/38/5/002.

[126] G. Mainfray and G. Manus. Multiphoton ionization of atoms. Reports on Progress in Physics, 54(3):1333-1372, 1991. doi: http://dx.doi.org/10.1088/0034-4885/54/ $10 / 002$. 
[127] Y.V. Afanasev, E.M. Belenov, and O.N. Krokhin. Cascade ionization of a gas by a powerful ultrashort pulse of light. Soviet Phys. JETP-USSR, 29(1):772, 1969.

[128] C. DeMichelis. Laser induced gas breakdown: A bibliographical review. IEEE Journal of Quantum Electronics, 5(4):188-202, 1969. doi: http://dx.doi.org/10. 1109/JQE.1969.1075758.

[129] Y.P. Raizer. Breakdown and Heating of Gases Under the Influence of a Laser Beam. Soviet Physics Uspekhi, 8(5):650-673, 1966. doi: http://dx.doi.org/10. 1070/PU1966v008n05ABEH003027.

[130] F. Docchio. Lifetimes of plasmas induced in liquids and ocular media by single nd:yag laser pulses of different duration. Europhysics Letters, 6(5):407-412, 2007. doi: http://dx.doi.org/10.1209/0295-5075/6/5/006.

[131] Y.M. Zolotovitskii, L.I. Korshunov, and V.A. Benderskii. Electron work function from metals in a liquid dielectric. Bulletin of the Academy of Sciences of the USSR, Division of chemical science, 21(4):760-763, 1972. doi: http://dx.doi.org/10.1007/ BF00854468.

[132] J.H. Bechtel, W. Lee Smith, and N. Bloembergen. Two-photon photoemission from metals induced by picosecond laser pulses. Physical Review B, 15(10):4557-4563, May 1977. doi: http://dx.doi.org/10.1103/PhysRevB.15.4557.

[133] E.M. Logothetis and P.L. Hartman. Laser-Induced Electron Emission from Solids: Many-Photon Photoelectric Effects and Thermionic Emission. Physical Review, 187(2):460-474, Nov 1969. doi: http://dx.doi.org/10.1103/PhysRev.187.460.

[134] R.A. Crowell, , and D.M. Bartels. Multiphoton ionization of liquid water with 3.0 - 5.0 ev photons. The Journal of Physical Chemistry, 100(45):17940-17949, 1996. doi: http://dx.doi.org/10.1021/jp9610978.

[135] B. Nikoobakht and M.A. El-Sayed. Preparation and growth mechanism of gold nanorods (NRs) using seed-mediated growth method. Chemistry of Materials, 15 (10):1957-1962, 2003. doi: http://dx.doi.org/10.1021/cm020732l. 
[136] C. Fernández-López, C. Mateo-Mateo, R.A. Álvarez-Puebla, J. Pérez-Juste, I. Pastoriza-Santos, and L.M. Liz-Marzán. Highly controlled silica coating of PEGcapped metal nanoparticles and preparation of SERS-encoded particles. Langmuir, 25(24):13894-13899, Dec 2009. doi: http://dx.doi.org/10.1021/la9016454.

[137] H. Baida, D. Christofilos, P. Maioli, A. Crut, N. Del Fatti, and F. Vallée. Surface plasmon resonance linear and nonlinear response in a single nanorod. Proc. of SPIE, 7033:703319, 2008. doi: http://dx.doi.org/10.1117/12.795124.

[138] A. Sommerfeld. Partial Differential Equations in Physics. Academic Press Inc., New York, 1949.

[139] S.V. Mutilin and T. Khasanov. The Refractive Index of Homogeneous SiO2 Thin Films. Optics and Spectroscopy, 105(3):461-465, Sep 2008. doi: http://dx.doi.org/ 10.1134/S0030400X0809018X.

[140] H. Baida, P. Billaud, S. Marhaba, D. Christofilos, E. Cottancin, a. Crut, J. Lermé, P. Maioli, M. Pellarin, M. Broyer, N. Del Fatti, F. Vallée, A. Sánchez-Iglesias, I. Pastoriza-Santos, and L.M. Liz-Marzán. Quantitative determination of the size dependence of surface plasmon resonance damping in single Ag@SiO2 nanoparticles. Nano Letters, 9(10):3463-3469, Oct 2009. doi: http://dx.doi.org/10.1021/ nl901672b.

[141] J. Lermé, H. Baida, C. Bonnet, M. Broyer, E. Cottancin, A. Crut, P. Maioli, N. Del Fatti, F. Vallée, and M. Pellarin. Size Dependence of the Surface Plasmon Resonance Damping in Metal Nanospheres. Journal of Physical Chemistry Letters, 1(19):2922-2928, 2010. doi: http://dx.doi.org/10.1021/jz1009136.

[142] E.A. Coronado and G.C. Schatz. Surface Plasmon Broadening for Arbitrary Shape Nanoparticles: A Geometrical Probability Approach. Journal of Chemical Physics, 119(7):3926-3934, 2003. doi: http://dx.doi.org/10.1063/1.1587686.

[143] C.R. Carey, T. Lebel, D. Crisostomo, J. Giblin, M. Kuno, and G.V. Hartland. Imaging and Absolute Extinction Cross-Section Measurements of Nanorods and Nanowires through Polarization Modulation Microscopy. Journal of Physical Chemistry C, 114(38):16029-16036, sep 2010. doi: http://dx.doi.org/10.1021/jp101891a. 
[144] J. Beuthan, O. Minet, J. Helfmann, M. Herrig, and G. Müller. The spatial variation of the refractive index in biological cells. Physics in Medicine and Biology, 41(3): 369-382, 1996. doi: http://dx.doi.org/10.1088/0031-9155/41/3/002.

[145] F.P. Bolin, L.E. Preuss, R.C. TayloR, and R.J. Ference. Refractive index of some mammalian tissues using a fiber optic cladding method. Applied Optics, 28(12): 2297-2303, Jun 1989. doi: http://dx.doi.org/10.1364/AO.28.002297.

[146] G. J. Tearney, M. E. Brezinski, B. E. Bouma, M. R. Hee, J. F. Southern, and J. G. Fujimoto. Determination of the refractive index of highly scattering human tissue by optical coherence tomography. Optics Letters, 20(21):2258-2260, Nov 1995. doi: http://dx.doi.org/10.1364/OL.20.002258.

[147] J.M. Schmitt and A. Knüttel. Model of optical coherence tomography of heterogeneous tissue. Journal of the Optical Society of America A, 14(6):1231-1242, Jun 1997. doi: http://dx.doi.org/10.1364/JOSAA.14.001231.

[148] T.Q. Qiu and C.L. Tien. Heat transfer mechanisms during short-pulse laser heating of metals. Journal of Heat Transfer, 115(4):835-841, 11 1993. doi: http://dx.doi. org $/ 10.1115 / 1.2911377$.

[149] D. Grand, A. Bernas, and E. Amouyal. Photoionization of aqueous indole: Conduction band edge and energy gap in liquid water. Chemical Physics, 44(1):73-79, 1979. doi: http://dx.doi.org/10.1016/0301-0104(79)80064-6.

[150] R. Lachaine, E. Boulais, E. Bourbeau, and M. Meunier. Effect of pulse duration on plasmonic enhanced ultrafast laser-induced bubble generation in water. Applied Physics A, 112(1):119-122, 2013. ISSN 1432-0630. doi: http://dx.doi.org/10.1007/ s00339-012-7210-1.

[151] C. Boutopoulos, A. Hatef, M. Fortin-Deschenes, and M. Meunier. Dynamic imaging of a single gold nanoparticle in liquid irradiated by off-resonance femtosecond laser. Nanoscale, 7:11758-11765, 2015. doi: http://dx.doi.org/10.1039/C5NR02721G.

[152] V. Juvé, M.F. Cardinal, A. Lombardi, A. Crut, P. Maioli, P. Pérez-Juste, L.M. Liz-Marzán, N. Del Fatti, and F. Vallée. Size-dependent surface plasmon resonance 
broadening in nonspherical nanoparticles: Single gold nanorods. Nano Letters, 13 (5):2234-2240, 2013. doi: http://dx.doi.org/10.1021/nl400777y.

[153] A.Q. Wu, I.H. Chowdhury, and X. Xu. Femtosecond laser absorption in fused silica: Numerical and experimental investigation. Physical Review B, 72:085128, Aug 2005. doi: http://dx.doi.org/10.1103/PhysRevB.72.085128.

[154] J.R. Gulley and T.E. Lanier. Model for ultrashort laser pulse-induced ionization dynamics in transparent solids. Physical Review B, 90:155119, Oct 2014. doi: http://dx.doi.org/10.1103/PhysRevB.90.155119.

[155] L. Sudrie, A. Couairon, M. Franco, B. Lamouroux, B. Prade, S. Tzortzakis, and A. Mysyrowicz. Femtosecond laser-induced damage and filamentary propagation in fused silica. Physical Review Letters, 89:186601, Oct 2002. doi: http://dx.doi. org/10.1103/PhysRevLett.89.186601.

[156] P. Grua, J. P. Morreeuw, H. Bercegol, G. Jonusauskas, and F. Vallée. Electron kinetics and emission for metal nanoparticles exposed to intense laser pulses. Physical Review B, 68:035424, Jul 2003. doi: http://dx.doi.org/10.1103/PhysRevB.68. 035424.

[157] S. Zafar, K. A. Conrad, Q. Liu, E.A. Irene, G. Hames, R. Kuehn, and J.J. Wortman. Thickness and effective electron mass measurements for thin silicon dioxide films using tunneling current oscillations. Applied Physics Letters, 67(7):1031-1033, 1995. doi: http://dx.doi.org/10.1063/1.114720.

[158] P. Audebert, Ph. Daguzan, A. Dos Santos, J. C. Gauthier, J. P. Geindre, S. Guizard, G. Hamoniaux, K. Krastev, P. Martin, G. Petite, and A. Antonetti. Space-time observation of an electron gas in sio2. Physical Review Letters, 73: 1990-1993, Oct 1994. doi: http://dx.doi.org/10.1103/PhysRevLett.73.1990.

[159] N. P. Blanchard, C. Smith, D. S. Martin, D. J. Hayton, T. E. Jenkins, and P. Weightman. High-resolution measurements of the bulk dielectric constants of single crystal gold with application to reflection anisotropy spectroscopy. physica status solidi (c), (8):2931-2937, 2003. doi: http://dx.doi.org/10.1002/pssc.200303828. 
[160] D.E. Palik. Handbook of Optical Constants of Solids. Orlando, Academic Press, 1985.

[161] Z.L. Wang, M.B. Mohamed, S. Link, and M.A. El-Sayed. Crystallographic facets and shapes of gold nanorods of different aspect ratios. Surface Science, 440(1-2): L809-L814, Oct 1999. doi: http://dx.doi.org/10.1016/S0039-6028(99)00865-1.

[162] E. Carbó-Argibay, B. Rodríguez-González, S. Gómez-Graña, A. Guerrero-Martínez, I. Pastoriza-Santos, J. Pérez-Juste, and L.M. Liz-Marzán. The crystalline structure of gold nanorods revisited: evidence for higher-index lateral facets. Angewandte Chemie International Edition, 49(49):9397-400, Dec 2010. doi: http://dx.doi.org/ 10.1002/anie.201004910.

[163] K.C. Vernon, A.M. Funston, C. Novo, D.E. Gómez, P. Mulvaney, and T.J. Davis. Influence of Particle-Substrate Interaction on Localized Plasmon Resonances. Nano Letters, 10(6):2080-2086, 2010. doi: http://dx.doi.org/10.1021/nl100423z.

[164] M.W. Knight, Y. Wu, J.B. Lassiter, P. Nordlander, and N.J. Halas. Substrates Matter: Influence of an Adjacent Dielectric on an Individual Plasmonic Nanoparticle. Nano Letters, 9(5):2188-2192, May 2009. doi: http://dx.doi.org/10.1021/ nl900945q.

[165] D.D. Evanoff, R.L. White, and G. Chumanov. Measuring the Distance Dependence of the Local Electromagnetic Field from Silver Nanoparticles. Journal of Physical Chemistry B, 108(5):1522-1524, feb 2004. doi: http://dx.doi.org/10.1021/ jp036899z.

[166] L.M. Liz-Marzán, J. Pérez-Juste, and I. Pastoriza-Santos. Plasmonics of Gold Nanorods. Considerations for Biosensing. Nanomaterials for Application in Medicine and Biology, pages 103-111, 2008. doi: http://dx.doi.org/10.1007/ 978-1-4020-6829-4_9.

[167] A. Vogel, J. Noack, K. Nahen, D. Theisen, S. Busch, U. Parlitz, D.X. Hammer, G.D. Noojin, B.A. Rockwell, and R. Birngruber. Energy balance of optical breakdown in water at nanosecond to femtosecond time scales. Applied Physics B, 68(2):271-280, 1999. doi: http://dx.doi.org/10.1007/s003400050617. 
[168] C. Voisin, D. Christofilos, N. Del Fatti, and F. Vallée. Environment effect on the acoustic vibration of metal nanoparticles. Physica B: Condensed Matter, 316-317 (1):89-94, May 2002. doi: http://dx.doi.org/10.1016/S0921-4526(02)00430-1.

[169] S. Ibrahimkutty, J. Kim, M. Cammarata, F. Ewald, J. Choi, H. Ihee, and A. Plech. Ultrafast Structural Dynamics of the Photocleavage of Protein Hybrid Nanoparticles. ACS Nano, 5(5):3788-3794, 2011. doi: http://dx.doi.org/10.1021/nn200120e.

[170] A. Barchanski, D. Funk, O. Wittich, C.h Tegenkamp, B.N. Chichkov, and C.L. Sajti. Picosecond Laser Fabrication of Functional Gold-Antibody Nanoconjugates for Biomedical Applications. Journal of Physical Chemistry C, 119(17):9524-9533, 2015. doi: http://dx.doi.org/10.1021/jp511162n.

[171] D. Werner, S. Hashimoto, and T. Uwada. Remarkable photothermal effect of interband excitation on nanosecond laser-induced reshaping and size reduction of pseudospherical gold nanoparticles in aqueous solution. Langmuir, 26(12):99569963, 2010. doi: http://dx.doi.org/10.1021/la100015t.

[172] D. Werner and S. Hashimoto. Controlling the pulsed-laser-induced size reduction of au and ag nanoparticles via changes in the external pressure, laser intensity, and excitation wavelength. Langmuir, 29(4):1295-1302, 2013. doi: http://dx.doi.org/ 10.1021/la3046143.

[173] H. Petrova, J. Perez Juste, I. Pastoriza-Santos, G.V. Hartland, L.M. Liz-Marzan, and P. Mulvaney. On the temperature stability of gold nanorods: comparison between thermal and ultrafast laser-induced heating. Phys. Chem. Chem. Phys., 8:814-821, 2006. doi: http://dx.doi.org/10.1039/B514644E.

[174] S. Hashimoto, T. Katayama, K. Setoura, M. Strasser, T. Uwada, and H. Miyasaka. Laser-driven phase transitions in aqueous colloidal gold nanoparticles under high pressure: picosecond pump-probe study. Physical Chemistry Chemical Physics, 18: 4994-5004, 2016. doi: http://dx.doi.org/10.1039/C5CP07395B.

[175] R.R. Letfullin, C. Joenathan, T.F. George, and V.P. Zharov. Laser-induced explosion of gold nanoparticles: potential role for nanophotothermolysis of cancer. 
Nanomedicine, 1(4):473-480, May 2006. doi: http://dx.doi.org/10.2217/17435889. 1.4.473.

[176] L. Delfour and T.E. Itina. Mechanisms of ultrashort laser-induced fragmentation of metal nanoparticles in liquids: Numerical insights. The Journal of Physical Chemistry C, 119(24):13893-13900, 2015. doi: http://dx.doi.org/10.1021/acs.jpcc. 5b02084.

[177] M. Strasser, K. Setoura, U. Langbein, and S. Hashimoto. Computational modeling of pulsed laser-induced heating and evaporation of gold nanoparticles. The Journal of Physical Chemistry C, 118(44):25748-25755, 2014. doi: http://dx.doi.org/10. 1021/jp508316v.

[178] I. N. Zavestovskaya, A. P. Kanavin, and S. D. Makhlysheva. Theoretical modeling of laser fragmentation of nanoparticles in liquid media. Bulletin of the Lebedev Physics Institute, 40(12):335-338, 2013. ISSN 1934-838X. doi: http://dx.doi.org/ $10.3103 / \mathrm{S} 1068335613120026$.

[179] D. Werner, A. Furube, T. Okamoto, and S. Hashimoto. Femtosecond laser-induced size reduction of aqueous gold nanoparticles: In situ and pumpâĽŠprobe spectroscopy investigations revealing coulomb explosion. The Journal of Physical Chemistry C, 115(17):8503-8512, 2011. doi: http://dx.doi.org/10.1021/jp112262u.

[180] F. Giammanco, E. Giorgetti, P. Marsili, and A. Giusti. Experimental and theoretical analysis of photofragmentation of au nanoparticles by picosecond laser radiation. The Journal of Physical Chemistry C, 114(8):3354-3363, 2010. doi: http://dx.doi.org/10.1021/jp908964t.

[181] V.L. Komolov, V.E. Gruzdev, S.G. Przhibelskii, and D.S. Smirnov. Dynamics of laser-induced damage of spherical nanoparticles by high-intensity ultrashort laser pulses. Optical Engineering, 51(12):121816-121816, 2012. doi: http://dx.doi.org/ 10.1117/1.OE.51.12.121816.

[182] Y.-J. Chen, M.-C. Lee, and C.-M. Wang. Dielectric function dependence on temperature for au and ag. Japanese Journal of Applied Physics, 53(8S2):08MG02, 2014. doi: http://dx.doi.org/10.7567/JJAP.53.08MG02. 
[183] N.M. Bulgakova, R. Stoian, A. Rosenfeld, E.E.B. Campbell, and I.V. Hertel. Model description of surface charging during ultra-fast pulsed laser ablation of materials. Applied Physics A, 79(4):1153-1155, 2004. doi: 10.1007/s00339-004-2692-0. 



\section{Acronyms}

ABC absorbing boundary condition. 38, 44, 47

AI avalanche ionization. xvii, 6, 7, 11, 12, 16, 24-26, 28-30, 32-34, 49, 51, 99

BEM boundary element method. 14

CB conduction band. 7, 32

CEM computational electromagnetic methods. 9

CTAB cetyltrimethylammonium bromide. 39, 40

DDA discrete-dipole approximation. 14

EM electromagnetic. 9, 12, 15, 37, 38, 46, 49, 51, 53, 55-57, 59, 60, 62, 99

FDTD finite difference time domain. 14

FE finite element. xvii, 37, 49, 56, 93, 102

FEM finite element method. 9, 14, 39, 46

fs femtosecond. 3, 11, 15, 29, 30, 32, 105

fwhm full-width at half-maximum. xviii, 45, 69, 71, 72

GNR gold nanorod. xviii, xix, 5, 8, 37, 39, 40, 42-47, 66-73, 83-91, 105, 107

HT heat transfer. xv, 24, 38, 51-53, 55 
IBA inverse Bremsstrahlung absorption. 5, 25, 26, 28, 29

IC ionization cascade. 25, 26, 29

LIB laser induced breakdown. 1-6

LIOB laser induced optical breakdown. xvii, xviii, 5-8, 10, 11, 13, 15-17, 24, 25, 37, $38,49,56,63,84-87,90,97,98,102,104$

LITB laser induced thermal breakdown. 5, 6

LSPR localized surface plasmon resonance. 2-5, 19, 20, 27

MPI multiphoton ionization. xvii, xix, 10-12, 15, 16, 24-27, 29, 30, 32-34, 49, 51, 57, $83,84,86-91,97,99$

NIR near-infrared. 2, 5, 56

ns nanosecond. 3, 13, 15, 29, 32, 33, 98, 104, 106

PDE partial differential equation. 9

PEC perfect electric conductor. $38,44,47,53,55$

PMC perfect magnetic conductor. 38, 44, 47, 53, 55

PML perfectly matched layer. $38,44,47,49-51,53,55$

ps picosecond. $3,13,15,29,30,105,106$

PTE photo-thermal emission. xix, 5-8, 10-12, 15, 16, 28-30, 32, 49, 51, 53, 57, 84, 86-91, 97, 99, 106

SEM scanning electron microscopy. 13

SMS spatial modulation spectroscopy. xvii, 4, 5, 39-43, 46, 69, 70, 72, 73

SPR surface plasmon resonance. 13, 14, 37, 42, 45, 66, 72, 73, 96

TEM transmission electron microscopy. v, xvii, 4, 5, 13, 39-44, 46, 47, 56, 66, 68, 96 
TI thermal ionization. 6, 10-12, 15, 16, 29, 30, 49, 51, 99

TTM two-temperature model. xv, 12, 22, 23, 38, 49, 51-53, 55, 56, 59, 62, 99

VB valence band. $7,25,28,32$ 
